c. 3

\title{
AN IMAGE PROCESSING TECHNIQUE FOR ACHIEVING LOSSY COMPRESSION OF DATA AT RATIOS IN EXCESS OF 100:1
} vy Corps ineers

\author{
by \\ Michael G. Ellis \\ Information Technology Laboratory \\ DEPARTMENT OF THE ARMY \\ Waterways Experiment Station, Corps of Engineers \\ 3909 Halls Ferry Road, Vicksburg, Mississippi 39180-6199
}

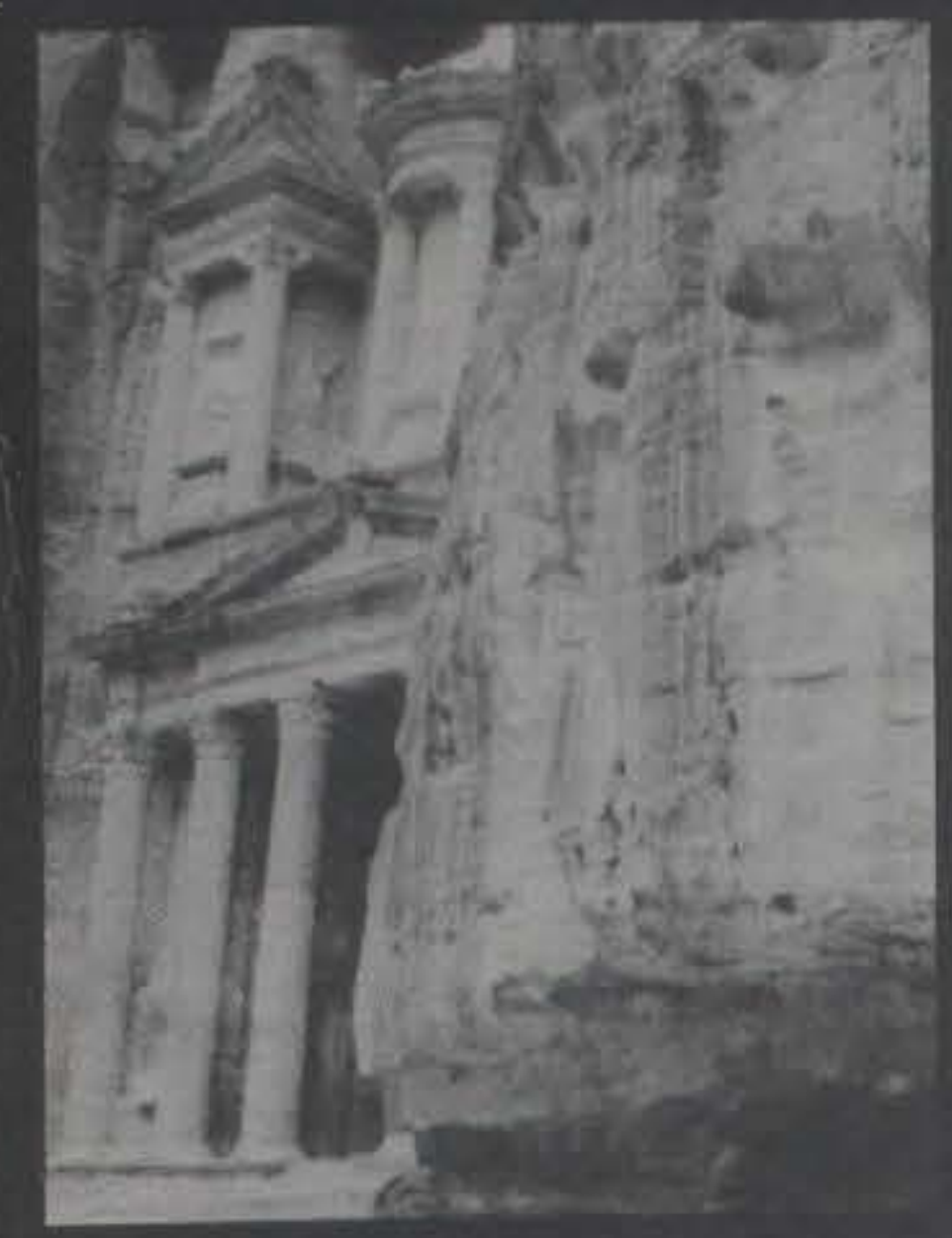

\section{US-CE-C PROPERT OF THE UNITED STATES GOVERNMENT}
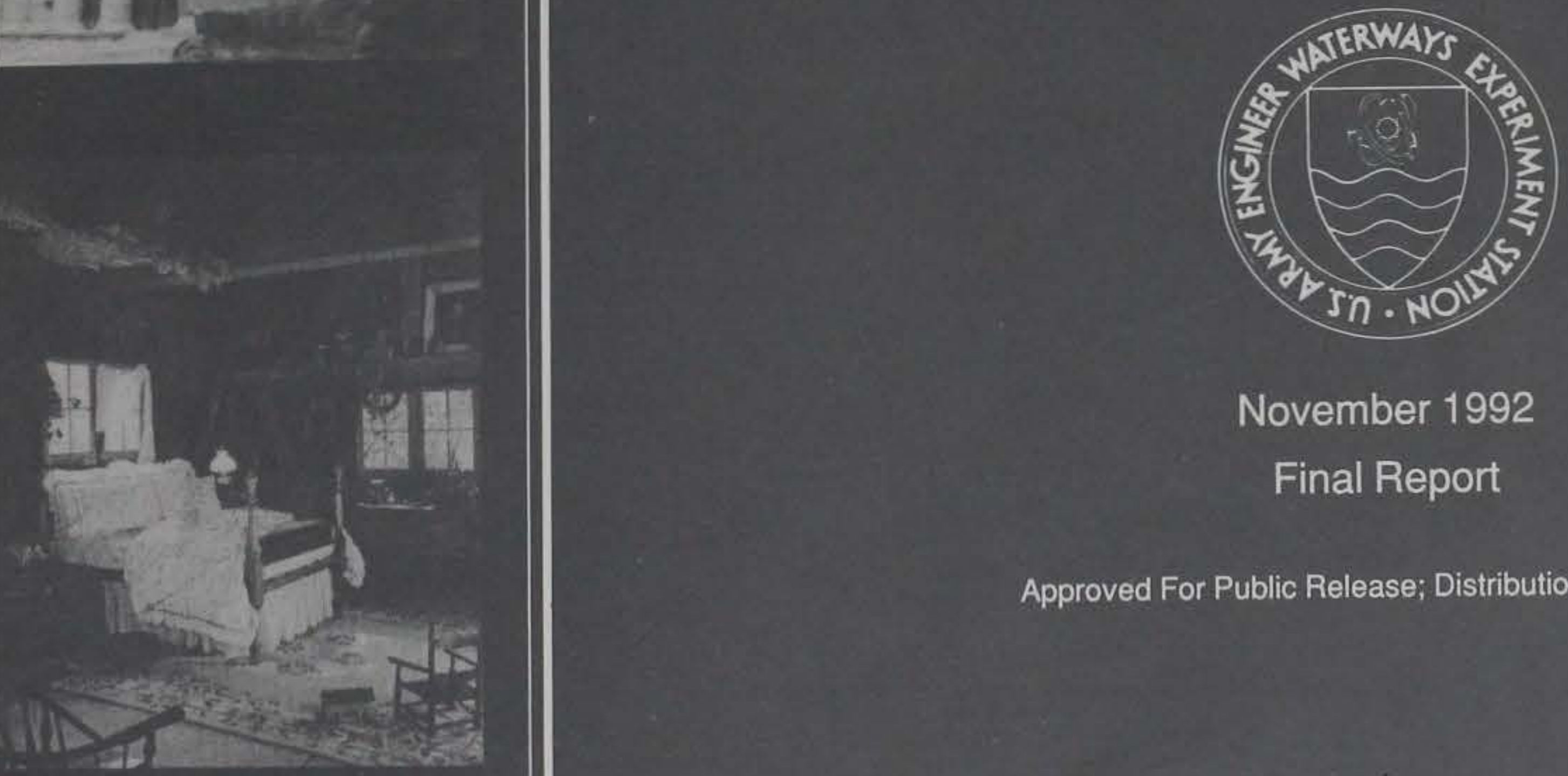

November 1992

Final Report

Approved For Public Release; Distribution Is Unlimited

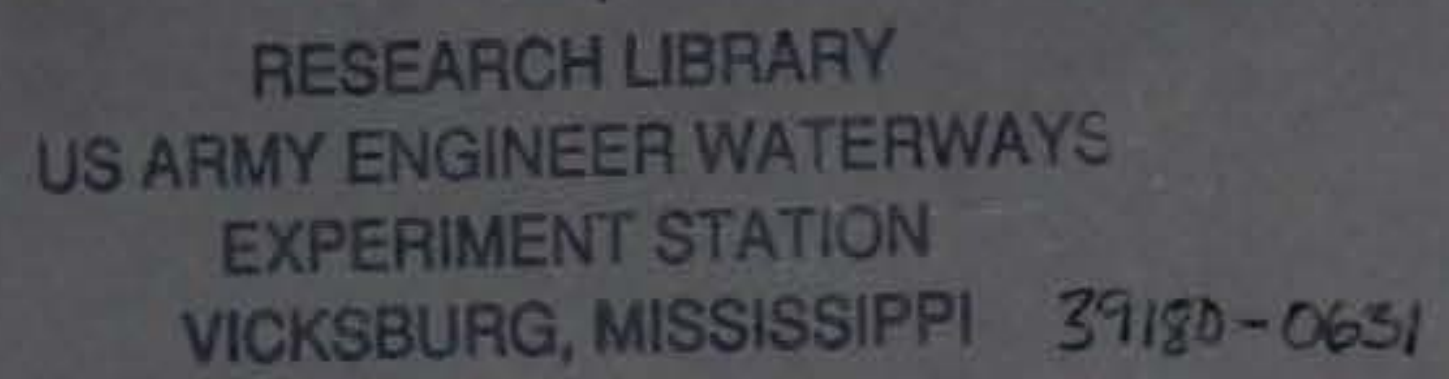

Prepared for DEPARTMENT OF THE ARMY US Army Corps of Engineers Washington, DC 20314-1000 
Public reporting burden for this collection of information is estimated to average 1 hour per response, including the time for reviewing instructions, searching existing data sources. gathering and maintaining the data needed, and completing and reviewing the collection of information. Send comments regarding this burden estimate or any other aspect of this Davis His Davis Highway. Suite 1204, Arlington, VA 22202-4302, and to the Office of Management and Budget, Paperwork Reduction Project (0704-0188), Washington, DC 20503.

\begin{tabular}{|l|l|l|} 
1. AGENCY USE ONLY (Leave blank) & 2. REPORT DATE & 3. REPORT TYPE AND DATES COVERED
\end{tabular}

4. TITLE AND SUBTITLE

November 1992

Final report

An Image Processing Technique for Achieving Lossy Compression of

Data at Ratios in Excess of 100:1

6. AUTHOR(S)

Michael G. Ellis

7. PERFORMING ORGANIZATION NAME(S) AND ADDRESS(ES)

US Army Engineer Waterways Experiment Station,

Information Technology Laboratory,

3909 Halls Ferry Road, Vicksburg, MS 39180-6199

9. SPONSORING/MONITORING AGENCY NAME(S) AND ADDRESS(ES)

US Army Corps of Engineers

Washington, DC 20314-1000

11. SUPPLEMENTARY NOTES

Available from National Technical Information Service, 5285 Port Royal Road, Springfield, VA 22161. 12a. DISTRIBUTION/AVAILABILITY STATEMENT

Approved for public release; distribution is unlimited.

\section{ABSTRACT (Maximum 200 words)}

This report presents a new method for image compression that achieves ratios in excess of 100:1. It begins as a tutorial of basic compression techniques that includes both lossless and lossy methods. The term "lossless" means that the compressed file can be reconstructed exactly, while "lossy" means that there is some loss of information in the reconstructed image. The most important components of the Joint Photographic Experts Group (JPEG) algorithm are targeted including Huffman encoding and the Cosine Transform. LZW, singular value decompostion, fractal compression, and other techniques are also discussed. The feasibility of using these methods for bandwidth reduction is analyzed relative to several High Definition TV (HDTV) standards. Finally, the cosine transform is modified to show how scanned images can be compressed 100:1 with little loss in quality. Several examples are presented.

\begin{tabular}{ll|}
\hline $\begin{array}{l}\text { 14. SUBJECT TERMS } \\
\text { Compression } \\
\text { Image processing } \\
\text { LOSSless }\end{array}$ & $\begin{array}{l}\text { Lossy } \\
\text { Video }\end{array}$ \\
\hline $\begin{array}{l}\text { 17. } \\
\text { SECURITY CLASSIFICATION REPORT } \\
\text { UNCLASSIFIED }\end{array}$ & $\begin{array}{l}\text { 18. SECURITY CLASSIFICATION } \\
\text { OF THIS PAGE } \\
\text { UNCLASSIFIED }\end{array}$ \\
\hline
\end{tabular}

\begin{tabular}{|l|l|}
\hline \multirow{2}{*}{$\begin{array}{l}\text { 19. SECURITY CLASSIFICATION } \\
\text { OF ABSTRACT }\end{array}$} & $\begin{array}{c}\text { 15. NUMBER OF PAGES } \\
217\end{array}$ \\
\cline { 2 - 3 } & 16. PRICE CODE \\
\hline $\begin{array}{l}\text { Standard Form 298 (ReV. 2-89) } \\
\text { Prescribed by ANSI Std. 239.18 } \\
\text { 298.102 }\end{array}$ \\
\hline
\end{tabular}




\section{Contents}

Preface $\ldots \ldots \ldots \ldots \ldots \ldots \ldots \ldots \ldots$

1-Lossless Coding Techniques $\ldots \ldots \ldots \ldots \ldots \ldots \ldots$

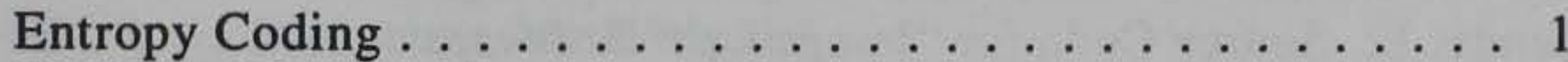

Huffman Compression . . . . . . . . . . . . . . 1

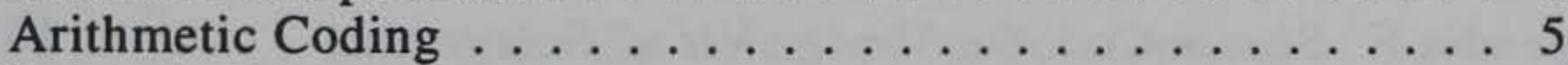

Lempel, Ziv, Welch (LZW) Compression . . . . . . . . . . 7

Lossless Compression Tests Results . . . . . . . . . . . . . 9

Exact Coding of Difference Files . . . . . . . . . 11

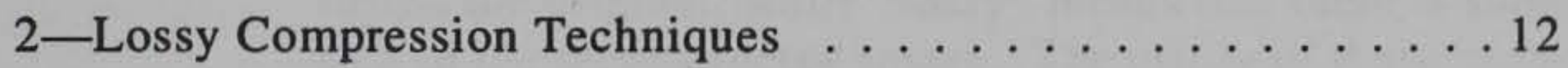

The Fourier Transform . . . . . . . . . . . . . . . 12

Representation of 8-Bit Images . . . . . . . . . . . . 13

The Discrete Cosine Transform (DCT) . . . . . . . . . 14

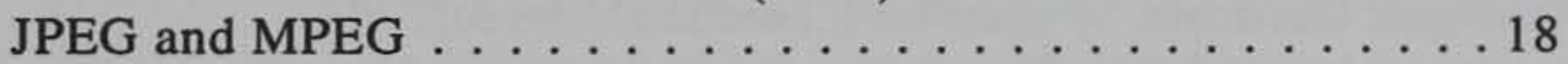

Singular Value Decomposition (SVD) . . . . . . . . . . 19

Fractal Compression . . . . . . . . . . . . . 24

3-Image Compression and Bandwidth Reduction . . . . . . . 26

Communication Standards . . . . . . . . . . . . . 26

Advanced Digital Television (ADTV) . . . . . . . . . . 29

Digital Spectrum Compatible (DSC) . . . . . . . . . 30

American Television Alliance Digicipher (ATVA) $\ldots \ldots .30$

4-Achieving High Compression Ratios $\ldots \ldots \ldots \ldots \ldots$. . . 32

Adaptive Cosine Transforms Using Pointers . . . . . . . . 32

Fourier Interpolation . . . . . . . . . . . . . . 37

The Relation of Spatial Subsampling to Zonal Filtering . . . . 38

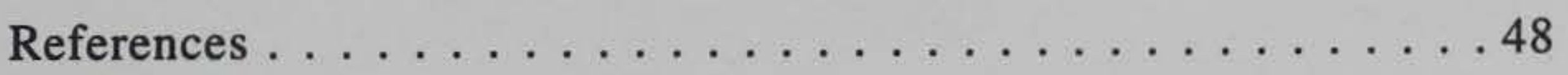

Bibliography $\ldots \ldots \ldots \ldots \ldots \ldots \ldots \ldots \ldots \ldots$

Appendix A: Compressions with the Cosine Transform and Singular

Value Decomposition (SVD) . . . . . . . . . A1 
Appendix B: "Image Lab" Software User's Guide $\ldots \ldots \ldots \ldots$ B1

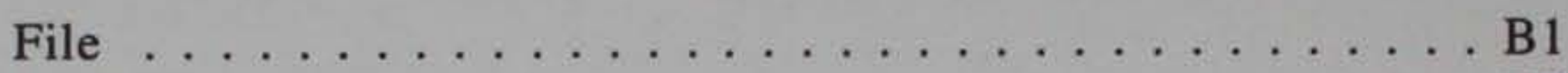

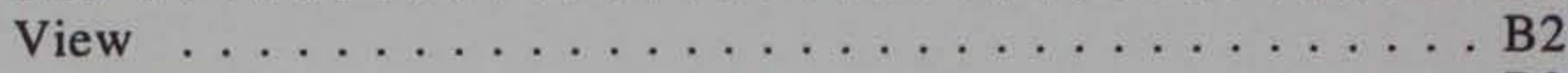

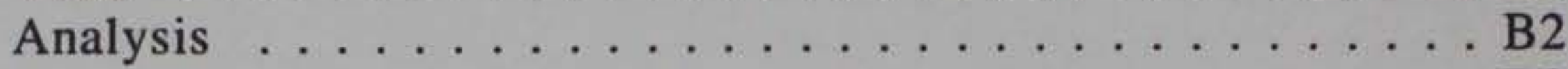

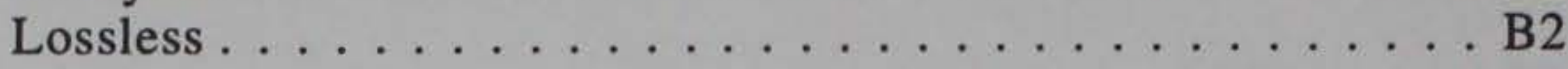

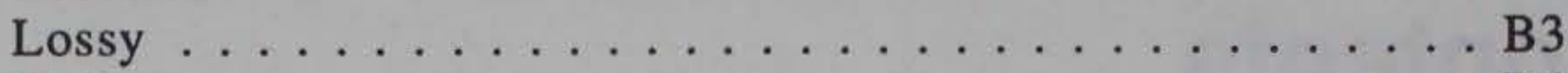

Options $\ldots \ldots \ldots \ldots \ldots \ldots \ldots \ldots \ldots \ldots \ldots \ldots \ldots \ldots \ldots \ldots \ldots \ldots$

Appendix C: "Image View" Software User's Guide $\ldots \ldots \ldots \ldots$ C1

File $\ldots \ldots \ldots \ldots \ldots \ldots \ldots \ldots \ldots \ldots \ldots \ldots \ldots \ldots \ldots \ldots \ldots$

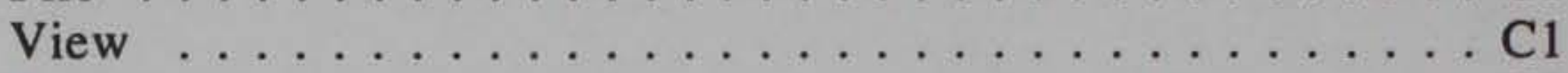

Options $\ldots \ldots \ldots \ldots \ldots \ldots \ldots \ldots \ldots \ldots \ldots \ldots \ldots \ldots \ldots \ldots$

Appendix D: Source Code for Individual Programs $\ldots \ldots \ldots$ D1

Appendix E: Source Code for "Image Lab" Software . . . . . E1

Appendix F: Source Code for "Image View" Software . . . . . F1 


\section{Preface}

This "Image Processing Technique for Achieving Lossy Compression of Data at Ratios in Excess of 100:1" documents a study by Mr. Michael G. Ellis, Electrical Engineer, Computer Science Division (CSD), Information Technology Laboratory (ITL), Waterways Experiment Station (WES), Vicksburg, Mississippi.

This report that describes the development of a new method for achieving compression ratios in excess of 100:1 begins by reviewing the standard lossless and lossy techniques that comprise existing algorithms. The term "lossless" means that the compressed file can be reconstructed to match the original, while "lossy" means that there is some loss of information in the reconstructed image. The utility of these methods to bandwidth reduction is analyzed relative to several High Definition TV (HDTV) standards.

Mr. Robert Moorhead, Professor of Electrical Engineering, Mississippi State University, contributed significantly to the success of the undertaking and provided many helpful suggestions. Mr. Roy Campbell, ITL, programmed the "Image Lab" software that was used in the research and development of these methods; Mr. Joel McAlister, ITL, also programmed many stand-alone utility programs and generated the hardcopy images shown in this document.

The work was accomplished at WES under the supervision of Dr. N. Radhakrishnan, Director, ITL, and Dr. Windell F. Ingram, Chief, CSD.

At the time of publication of this report, the Director of WES was Dr. Robert W. Whalin. The Commander was COL Leonard G. Hassell, EN. 


\section{Lossless Coding Techniques}

\section{Entropy Coding}

The term "entropy" is borrowed from thermodynamics and has a similar meaning. The higher the entropy of a message, the more information it contains. The entropy of a data file is defined as the information per byte for that particular file. If the 8-bit ASCII character set that makes up a data or image file is not uniformly distributed, then the entropy will be less than 8 bits/byte. In entropy coding, the goal is to encode a block of $M$ pixels each containing $B$ bits, for a total of $M^{*} B$ bits. The entropy for any general file is given as

$$
H=-\sum_{i=1}^{M}\left[p_{i} \log _{2}\left(p_{i}\right)\right]
$$

In an 8-bit character set, there are 256 values that can be represented by a single byte. The probability, $p_{i}$, is the total occurrences of the $\mathrm{i}_{\text {th }}$ byte divided by the total number of bytes in the data file.

The program, ENTROPY.BAS, uses Equation 1 to estimate the amount of compression achievable by entropy coding. ENTROPY.BAS works only under QuickBasic 4.5 because of the "open binary" statement in line 70. The $C$ language version, ENTROPY.C, is provided to illustrate the syntax differences between BASIC and C. Most compression programs are written in $\mathrm{C}$ since the more optimized $\mathrm{C}$ compiler produces executables which are 10 times faster than compiled BASIC.

\section{Huffman Compression}

This classic compression technique, introduced in 1952 by David A. Huffman, is perhaps the most well-known for entropy compression. It 
achieves the minimum amount of redundancy possible in a fixed set of variable-length codes and does not mean that Huffman encoding is an optimal encoding method. It means that it provides the best approximation for encoding symbols when using fixed-width codes.

The entropy of a data file is given by Equation 1. In general, $\log _{2}\left(p_{i}\right)$ will not be an integer so that the achieved data rate exceeds $H$ bits/pixel; however, the data rate can be made to asymptotically approach $H$ with increasing block size. Huffman encoding operates similar to the Morse code in which the most frequently used symbols are given the shortest code. However, it is not obvious how to optimally assign a set of varying length codes to a set of symbols to be transmitted. Huffman encoding optimizes by assigning a varying length code to a set of symbols based on the probability of occurrence. This method does not exclude the possibility of other lossless techniques producing a higher compression rate.

Suppose our total set of characters is represented by eight ASCII values with probabilities in Table 1. Eight ASCII values can be represented by 3 bits/pixel; however, for the given probabilities of occurrence, the entropy is only 2.6984 bits/pixel. Therefore, Huffman encoding can provide a compression scheme with a bit rate approaching 2.6984 bits/pixel.

\begin{tabular}{|l|l|l|}
\hline \multicolumn{3}{|l|}{$\begin{array}{l}\text { Table 1 } \\
\text { Sample 3-Bit Character Set with Probabilities of Occurrence }\end{array}$} \\
\hline Character \# & Probability & Entropy (H) \\
\hline 1 & 0.30 & $0.521089=-0.3 \log _{2}(0.3)$ \\
\hline 2 & 0.23 & $0.487667=-0.23 \log _{2}(0.23)$ \\
\hline 3 & 0.15 & $0.410544=-0.15 \log _{2}(0.15)$ \\
\hline 4 & 0.08 & $0.291508=-0.08 \log _{2}(0.08)$ \\
\hline 5 & 0.06 & $0.243533=-0.06 \log _{2}(0.06)$ \\
\hline 6 & 0.06 & $0.243533=-0.06 \log _{2}(0.06)$ \\
\hline 7 & 0.06 & $0.243533=-0.06 \log _{2}(0.06)$ \\
\hline 8 & 0.06 & $0.243533=-0.06 \log _{2}(0.06)$ \\
\hline & 1.0 & Total entropy $=2.6984$ bits/pixel \\
\hline
\end{tabular}

The Huffman code is a variable length code in which no code can be a prefix for any other Huffman code. Thus, unambiguous decoding of the transmitted data stream is allowed.

The Huffman codebook that is generated by the tree algorithm must be transmitted prior to the actual data in order to set up the decoder. Programs for Huffman encoding require two passes over the data. Probabilities are 
generated in the first pass, and the actual Huffman compressed file is generated during the second pass. The following is an outline of the Huffman coding algorithm:

$a$. Arrange the symbol probabilities $p_{i}$ in a decreasing order, and consider them as leaf nodes of a tree.

$b$. While there is more than one node:

(1) Merge the two nodes with smallest probability to form a new node whose probability is the sum of the two merged nodes.

(2) Arbitrarily assign 1 and 0 to each pair of branches merging into a node.

c. Read sequentially from the root node to the leaf node where the symbol is located.

The actual achieved compressed data rate is 2.71 bits/pixel and can be determined as follows:

\begin{tabular}{|l||}
\hline $0.30 * 2$ bits $=0.60$ \\
\hline $0.23 * 2$ bits $=0.46$ \\
\hline $0.15 * 3$ bits $=0.45$ \\
\hline $0.08 * 3$ bits $=0.24$ \\
\hline $0.06 * 4$ bits $=0.24$ \\
\hline $0.06 * 4$ bits $=0.24$ \\
\hline $0.06 * 4$ bits $=0.24$ \\
\hline $0.06 * 4$ bits $=0.24$ \\
\hline Total $=2.71$ bits $/$ symbol \\
\hline
\end{tabular}

The coding sequence from Figures 1 and 2 follows:

\begin{tabular}{|l|l|l|}
\hline Character \# & Probability & Huffman Codeword \\
\hline 1 & 0.30 & 10 \\
\hline 2 & 0.23 & 00 \\
\hline 3 & 0.15 & 110 \\
\hline 4 & 0.08 & 010 \\
\hline 5 & 0.06 & 0110 \\
\hline 6 & 0.06 & 0111 \\
\hline 7 & 0.06 & 1110 \\
\hline 8 & 0.06 & 1111 \\
\hline
\end{tabular}




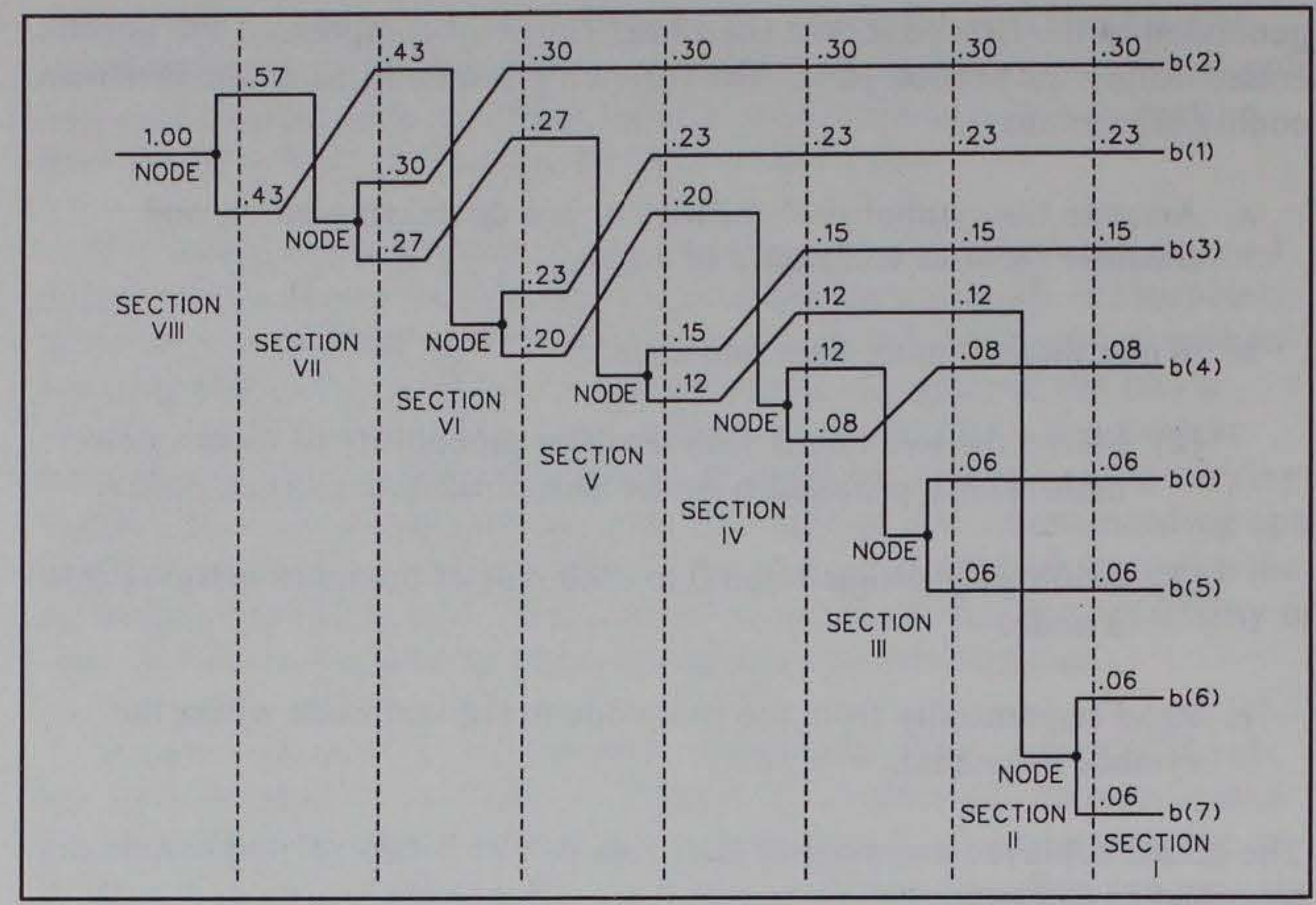

Figure 1. Construction of a binary Huffman code proceeds from right to left. At each section the tow bottom-most branches are combined to form a node and followed by a reordering of probabilities into descending order. These probabilities are then used to start the next section

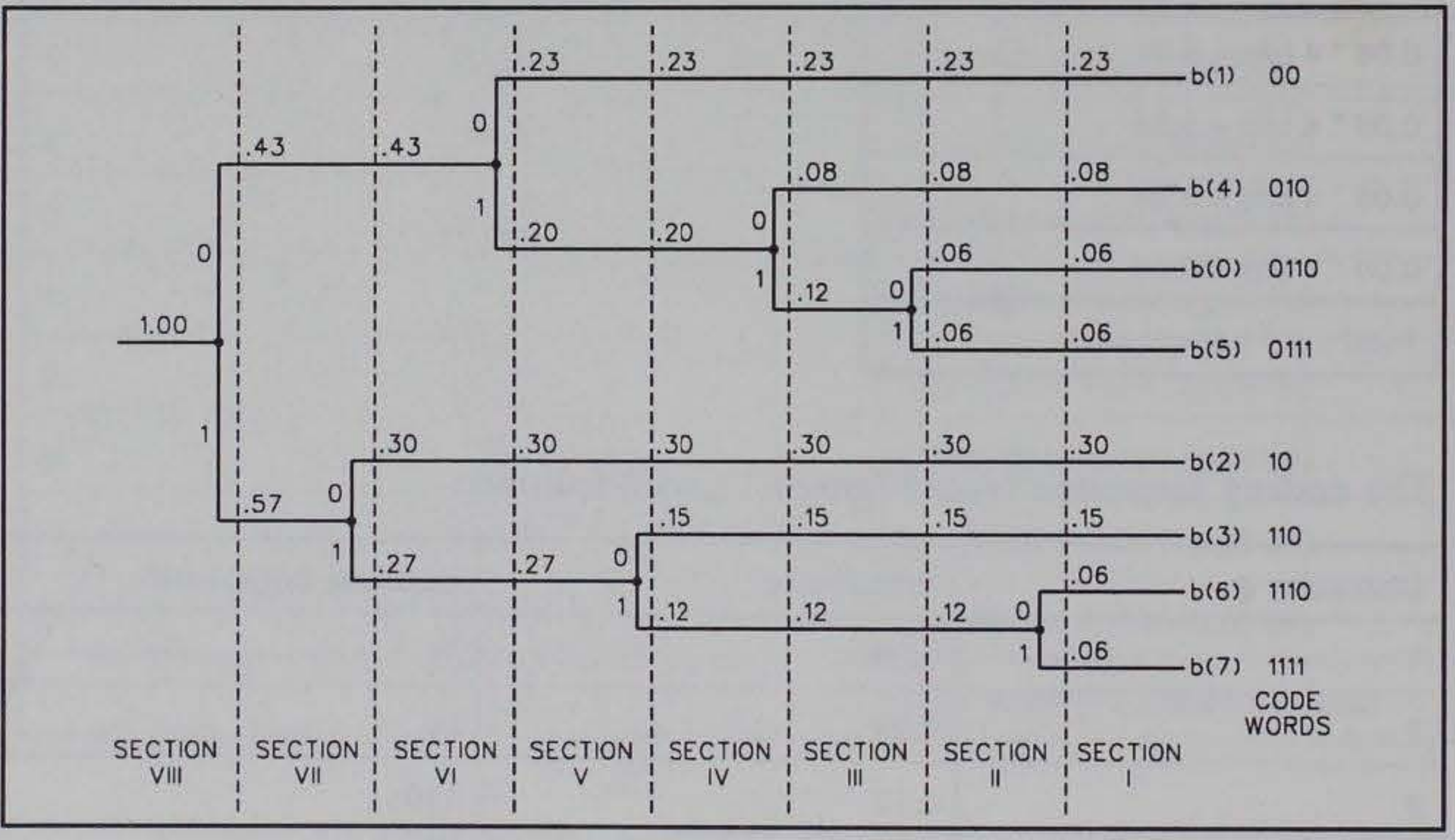

Figure 2. After the code construction, the tree is rearranged to eliminate crossovers, and coding proceeds from left to right. At each node a step-up produces a zero and a step-down a one. Resulting code words are shown at the right for each of the eight levels. Note that no code word is a prefix of any other code word 
In high performance data compression, Huffman encoding faces a significant problem. The program has to pass a complete copy of the coding statistics to the expansion program. For a Huffman Order- 0 encoder, there are $8 \mathrm{bits} / \mathrm{byte}$, and the probability table that is passed to the decoder may occupy as little as 256 bytes. However, a Huffman Order- 0 encoder sometimes cannot achieve compression rates close to the entropy given in Equation 1. A Huffman Order-1 encoder groups the symbols into 16-bit words but boosts the statistics table from 256 to 65,536 bytes. Though compression ratios will undoubtedly improve when moving to order-1, the overhead of passing the statistics table will probably nullify any gains.

Adaptive Huffman encoding solves this situation without paying any penalty for added statistics. It does so by adjusting the Huffman tree based on data previously seen, with no knowledge about future statistics. When using an adaptive model, the pixel information does not have to be scanned once in order to generate statistics. Instead, the statistics are continually modified as new characters are read in and encoded. A more detailed discussion of Adaptive Huffman coding can be obtained from a study by Nelson (1991).

\section{Arithmetic Coding}

Arithmetic coding is a lossless technique that can compress below the entropy level. It generally provides 10 to 20 percent better compression than Huffman. The output from an arithmetic process is a single number less than 1 and greater than or equal to 0 . This single number can be uniquely decoded to exactly recreate the original byte stream.

To construct the output number, the symbols are assigned a set of probabilities. The message "KING HENRY" would have a probability distribution as follows:

\begin{tabular}{|l|l|}
\hline Character & Probability \\
\hline Space & $1 / 10$ \\
\hline E & $1 / 10$ \\
\hline G & $1 / 10$ \\
\hline H & $1 / 10$ \\
\hline I & $1 / 10$ \\
\hline K & $1 / 10$ \\
\hline N & $2 / 10$ \\
\hline R & $1 / 10$ \\
\hline Y & $1 / 10$ \\
\hline
\end{tabular}


Individual characters can now be assigned along a probability line as follows:

\begin{tabular}{|l|l|l|}
\hline Character & Probability & Range \\
\hline Space & $1 / 10$ & $0.00 \geq r>0.10$ \\
\hline E & $1 / 10$ & $0.10 \geq r>0.20$ \\
\hline G & $1 / 10$ & $0.20 \geq r>0.30$ \\
\hline H & $1 / 10$ & $0.30 \geq r>0.40$ \\
\hline I & $1 / 10$ & $0.40 \geq r>0.50$ \\
\hline K & $1 / 10$ & $0.50 \geq r>0.60$ \\
\hline N & $2 / 10$ & $0.60 \geq r>0.80$ \\
\hline R & $1 / 10$ & $0.80 \geq r>0.90$ \\
\hline Y & $1 / 10$ & $0.90 \geq r>1.00$ \\
\hline
\end{tabular}

The most significant portion of an arithmetic encoded message belongs to the first symbol, or " $\mathrm{K}$," in the message "King Henry." To decode the first character properly, the final encoded message has to be a number greater than or equal to 0.5 , and less than 0.6 . Therefore, the low end for this range is 0.5 , and the high end is 0.6 . During the rest of the encoding process, each new symbol will further restrict the possible range of the output number. The next character to be encoded, the letter "I," has a range from 0.4 to 0.5 in the subrange 0.5 to 0.6 . Applying this logic will further restrict the number to the range of 0.54 to 0.55 . Continuing this process results in the final low value of 0.5464063556 , which will uniquely decode into "KING HENRY," as tabulated below.

\begin{tabular}{|l|l|l|}
\hline New Character & Low Value & High Value \\
\hline K & 0.5 & 0.6 \\
\hline I & 0.54 & 0.55 \\
\hline N & 0.546 & 0.548 \\
\hline G & 0.5464 & 0.5466 \\
\hline Space & 0.54640 & 0.54642 \\
\hline H & 0.546406 & 0.546408 \\
\hline E & 0.5464062 & 0.5464064 \\
\hline N & 0.54640632 & 0.54640636 \\
\hline R & 0.546406352 & 0.546406356 \\
\hline Y & 0.5464063556 & 0.546406356 \\
\hline
\end{tabular}


Arithmetic coding is best accomplished using 32-bit binary integral math. The decimal point is implied at the left side of the word. The initial value of HIGH is \$FFFFFFFF, and LOW is $\$ 00000000$. Decoding is accomplished by first transmitting a table of statistics to the decoder and then by transmitting a series of integers to be decoded. Typically, arithmetic coding is not used in shareware since IBM holds the patent for this technique.

\section{Lempel, Ziv, Welch (LZW) Compression}

The LZW compression is related to two compression techniques known as LZ77 and LZ78. LZ77 is a "sliding window" process in which the dictionary consists of a set of fixed-length phrases found in a "window" in the previously processed text. The size of the window is generally somewhere between $2-\mathrm{K}$ and $16-\mathrm{K}$ bytes, with the maximum phrase length ranging from perhaps 16 to 64 bytes. LZ78 takes a completely different approach to building a dictionary. Instead of using fixed-length phrases from a window in the text, LZ78 builds phrases up one symbol at a time, adding a new symbol to an existing phrase when a match occurs.

The LZ77 technique has a major performance bottleneck. When encoding, it has to perform string comparisons against the look-ahead buffer for every position in the text window. As LZ77 tries to improve compression performance by increasing the size of the window (and thus the dictionary), this performance bottleneck only gets worse. LZSS seeks to avoid some of the performance problems in the LZ77 algorithm. Under LZ77, the phrases in the text window were stored as a single contiguous block of text, with no other organization on top of it. LZSS still stores text in contiguous windows, but it creates an additional data structure that improves on the organization of the phrases. As each phrase passes out of the lookahead buffer and into the encoded portion of the test windows, LZSS adds the phrase to a tree structure. The savings created by using the tree not only make the compression side of the algorithm much more efficient but also encourage experimentation with longer window sizes. Doubling the size of the text window now might only cause a small increase in the compression time; whereas, before it would have doubled it.

The LZ78 technique is similar to LZ77 but abandons the concept of a text window. Under LZ77, the dictionary of phrases was defined by a fixed window of previously seen text. With LZ78, the dictionary is a potentially unlimited list of previously seen phrases. An improved LZ78 algorithm in which the phrase dictionary is preloaded with single-symbol phrases equal to the number of symbols in the alphabet is LZW. However, LZW never outputs single characters, only phrases.

The LZW compression is shown using "BET BE BEE BED BEG" as the input stream. The quotation marks are used only to indicate that the character stream begins with a "space." The first 256 codes ( 0 to 255 ) are 
not shown since the following tabulation is generated by LZW as an appendum to the first 256 characters.

\begin{tabular}{|c|c|c|c|}
\hline Step & Character & Code Output & New Code \\
\hline 1 & Space & none & [already in table] \\
\hline 2 & B & ASCII for space & space B at (256) \\
\hline 3 & $\mathrm{E}$ & ASCII for B & BE at (257) \\
\hline 4 & $T$ & ASCII for $E$ & ET at (258) \\
\hline 5 & Space & ASCII for $T$ & T space at (259) \\
\hline 6 & B & none & [space B already in table] \\
\hline 7 & E & 256 & space BE at (260) \\
\hline 8 & Space & ASCII for $E$ & E space at (261) \\
\hline 9 & B & none & [space B already in table] \\
\hline 10 & E & none & [space BE already in table] \\
\hline 11 & $E$ & 260 & BEE space (262) \\
\hline 12 & Space & none & [E space already in table] \\
\hline 13 & B & 261 & E space B at (263) \\
\hline 14 & E & none & [BE already in table] \\
\hline 15 & D & 257 & BED at (264) \\
\hline 16 & Space & D & D space at (265) \\
\hline 17 & B & none & [space B already in table] \\
\hline 18 & E & none & [space BE already in table] \\
\hline 19 & $T$ & 260 & BET at (266) \\
\hline 20 & $\langle E O F\rangle$ & ASCII for $T$ & \\
\hline
\end{tabular}

The first seven steps in this sequence are explained as follows:

Step 1: A "space" is read. The ASCII value for "space" is already contained in the table. No action is taken.

Step 2: A "B" is read. There is no "space B" combination in the table. The ASCII value for "space" is output, and "space B" is assigned code 256 . 
Step 3: An "E" is read. There is no "BE" combination in the table, so " $\mathrm{BE}$ " is assigned code 257 and the ASCII value for " $\mathrm{B}$ " is output.

Step 4: A "T" is read. There is no "ET" combination in the table, so "ET" is assigned code 258 and the ASCII value for " $E$ " is output.

Step 5: A "space" is read. There is no "T space" combination in the table, so "T space" is assigned code 259 and the ASCII value for " $T$ " is output.

Step 6: A "B" is read. The combination "space B" already exists in the table, so no action is required.

Step 7: An "E" is read. The combination "space BE" is assigned code 260 , and code 256 for "space B."

The LZW decompressor takes the stream of codes from the compression algorithm and uses them to recreate the exact input stream. One reason for the efficiency of the LZW algorithm is that it does not need to pass the dictionary to the decompressor since the table can be built exactly as it was during compression, using the input stream as data. This step is possible because the compression algorithm always outputs the phrase and character components of a code before it uses it in the output stream, so the compressed information is not burdened with carrying a large dictionary. Typically 12-bit codes words will be used in an LZW algorithm for 4,096 possible phrases to accommodate the standard 256 eightbit character set plus the additional phrases that are constructed as the input stream is processed. The patent for LZW is assigned to Unisys, which has made public its intention to protect its intellectual property rights. The LZW compression is defined as part of the CCITT V.42 bis specification, and Unisys has defined specific terms under which it will license the algorithm to modem manufacturers. It has not stated that it will apply the same terms to any parties manufacturing other types of products.

\section{Lossless Compression Tests Results}

At the U.S. Army Engineer Waterways Experiment Station (WES), tests were run on six lossless compression routines. The results of the tests are given in Table 2. The compression time and performance is given for each file and package. The first three files are executable programs. The next two are text files. JBARB2Y.COL and JGOLDY.COL are 8-bit grayscale images. The last four files are 8-bit RGB color images. The source and compiled codes for the following programs are available from a floppy disk entitled "Software listings" (1991). ARC, ZIP, and COMPRESS implement variations of the LZW algorithm. The highest performance package (in terms of compression) is LHARC by 


\begin{tabular}{|c|c|c|c|c|c|c|}
\hline \multicolumn{7}{|c|}{$\begin{array}{l}\text { Table } 2 \\
\text { Lossless Compression Test Results }\end{array}$} \\
\hline FILE & ARC & LHARC & 200 & ZIP & COMPRESS & ENTROPY \\
\hline WP.EXE & $2.55 \mathrm{~s}$ & $6.35 \mathrm{~s}$ & $5.11 \mathrm{~s}$ & $3.53 \mathrm{~s}$ & $23.55 \mathrm{~s}$ & \\
\hline 244,736 & 222,275 & 175,828 & 223,621 & 176,564 & 230,025 & 222,383 \\
\hline $100 \%$ & $90.8 \%$ & $71.8 \%$ & $91.4 \%$ & $72.1 \%$ & $94.0 \%$ & $90.8 \%$ \\
\hline WS.EXE & $1.78 \mathrm{~s}$ & $2.40 \mathrm{~s}$ & $2.20 \mathrm{~s}$ & $1.34 \mathrm{~s}$ & $5.84 \mathrm{~s}$ & \\
\hline 78,336 & 69,158 & 60,704 & 75,191 & 61,982 & 74,007 & 69,214 \\
\hline $100 \%$ & $88.3 \%$ & $77.5 \%$ & $96.0 \%$ & $79.1 \%$ & $94.5 \%$ & $88.4 \%$ \\
\hline PROCOMM.EXE & $1.85 \mathrm{~s}$ & $4.60 \mathrm{~s}$ & $2.78 \mathrm{~s}$ & $2.90 \mathrm{~s}$ & $11.30 \mathrm{~s}$ & \\
\hline 165,296 & 104,312 & 82,072 & 103,510 & 80,782 & 105,895 & 129,729 \\
\hline $100 \%$ & $63.1 \%$ & $49.7 \%$ & $62.6 \%$ & $48.9 \%$ & $64.1 \%$ & $78.5 \%$ \\
\hline SILVERD.DOC & $7.18 \mathrm{~s}$ & $35.17 \mathrm{~s}$ & $11.29 \mathrm{~s}$ & $24.71 \mathrm{~s}$ & $62.44 \mathrm{~s}$ & \\
\hline $1,096,064$ & 403,771 & 365,080 & 412,111 & 359,696 & 363,231 & 586,292 \\
\hline $100 \%$ & $36.8 \%$ & $33.3 \%$ & $37.6 \%$ & $32.8 \%$ & $33.1 \%$ & $53.5 \%$ \\
\hline REGISTER.TXT & $0.66 \mathrm{~s}$ & $0.66 \mathrm{~s}$ & $0.69 \mathrm{~s}$ & $0.55 \mathrm{~s}$ & $1.00 \mathrm{~s}$ & \\
\hline 6,801 & 3,478 & 2,903 & 3,645 & 2,946 & 3,476 & 4,004 \\
\hline $100 \%$ & $51.1 \%$ & $42.7 \%$ & $53.6 \%$ & $43.2 \%$ & $51.1 \%$ & $58.9 \%$ \\
\hline BARB2Y.COL & $6.40 \mathrm{~s}$ & $11.71 \mathrm{~s}$ & $10.59 \mathrm{~s}$ & $5.92 \mathrm{~s}$ & $38.78 \mathrm{~s}$ & \\
\hline 403,200 & 377,351 & 352,790 & 412,625 & 373,014 & 381,029 & 377,173 \\
\hline $100 \%$ & $93.6 \%$ & $87.5 \%$ & $102 \%$ & $92.5 \%$ & $94.5 \%$ & $93.5 \%$ \\
\hline GOLDY.COL & $4.40 \mathrm{~s}$ & $11.03 \mathrm{~s}$ & $7.53 \mathrm{~s}$ & $6.40 \mathrm{~s}$ & $36.9 \mathrm{~s}$ & \\
\hline 403,200 & 373,323 & 339,233 & 372,283 & 350,122 & 350,123 & 380,240 \\
\hline $100 \%$ & $92.5 \%$ & $84.1 \%$ & $92.3 \%$ & $86.8 \%$ & $86.8 \%$ & $94.3 \%$ \\
\hline BARB.RGB & $3.21 \mathrm{~s}$ & $13.51 \mathrm{~s}$ & $4.28 \mathrm{~s}$ & $9.54 \mathrm{~s}$ & $25.6 \mathrm{~s}$ & \\
\hline 403,200 & 150,193 & 152,410 & 152,041 & 165,471 & 146,998 & 248,399 \\
\hline $100 \%$ & $37.3 \%$ & $37.8 \%$ & $37.7 \%$ & $41.0 \%$ & $36.5 \%$ & $61.6 \%$ \\
\hline GOLD.RGB & $2.73 \mathrm{~s}$ & $14.78 \mathrm{~s}$ & $3.84 \mathrm{~s}$ & $13.73 \mathrm{~s}$ & $20.72 \mathrm{~s}$ & \\
\hline 403,200 & 122,381 & 124,775 & 119,857 & 135,986 & 119,843 & 235,154 \\
\hline $100 \%$ & $30.3 \%$ & $30.9 \%$ & $29.7 \%$ & $33.7 \%$ & $29.7 \%$ & $58.3 \%$ \\
\hline BOATS.RGB & $2.65 \mathrm{~s}$ & $19.79 \mathrm{~s}$ & $3.53 \mathrm{~s}$ & $14.87 \mathrm{~s}$ & $17.92 \mathrm{~s}$ & \\
\hline 403,200 & 94,844 & 98,351 & 95,231 & 106,958 & 96,405 & 204,358 \\
\hline $100 \%$ & $23.5 \%$ & $24.4 \%$ & $23.6 \%$ & $26.5 \%$ & $23.9 \%$ & $50.6 \%$ \\
\hline BOARD.RGB & $2.67 \mathrm{~s}$ & $15.93 \mathrm{~s}$ & $3.59 \mathrm{~s}$ & $14.29 \mathrm{~s}$ & $19.09 \mathrm{~s}$ & \\
\hline 403,200 & 107,201 & 108,918 & 107,458 & 118,593 & 105,775 & 218,287 \\
\hline $100 \%$ & $26.6 \%$ & $27.0 \%$ & $26.7 \%$ & $29.4 \%$ & $26.2 \%$ & $54.1 \%$ \\
\hline
\end{tabular}


Haruyasu Yoshizaki which is built on an LZ77 algorithm using a dictionary-based method on a sliding window that moves through the text. All of these packages are compared against standard entropy coding (Table 2) which consistently provided the worst compression.

Under each routine in Table 2, compression time is shown in seconds for an IBM compatible $486 / 33 \mathrm{Mhz}$ PC. The next two rows show the file size after compression and the size of the compressed file as a percentage of the original.

\section{Exact Coding of Difference Files}

If a pixel by pixel difference file is created from an original image, then Huffman encoding can be used to give a lossless compression at ratios that can exceed the other lossless techniques in Table 2. The first pixel was retained as its original value, and all the other 8-bit values represent the differences between the previous pixel and the present pixel. The following code section shows how overflow programming can avoid 9-bit differences:

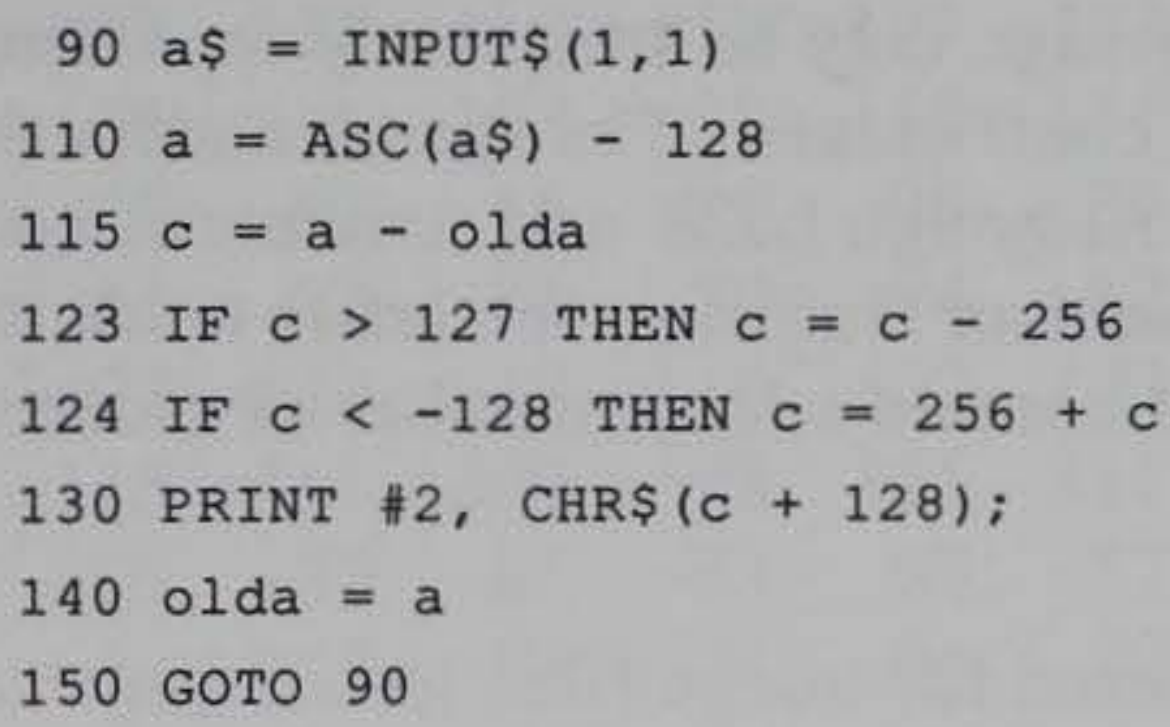

\begin{tabular}{|l|l|}
\hline File $(\mathbf{4 0 3 , 2 0 0 )}$ & Compressed Size \\
\hline JBARB2Y.COL & 300,681 \\
\hline JGOLDY.COL & 257,544 \\
\hline BARB.RGB & 164,107 \\
\hline GOLD.RGB & 125,458 \\
\hline BOATS.RGB & 106,120 \\
\hline BOARD.RGB & 127,346 \\
\hline
\end{tabular}




\section{Lossy Compression Techniques}

\section{The Fourier Transform}

Lossy compression methods are useful for image compression and are often based on the Fourier transform. In general, a two-dimensional (2-D) Fourier transform of an image will produce a large number of coefficients close to zero. For example, if 95 percent of the transform coefficients can be approximated by zero, then the image may be adequately represented using only 5 percent of the Fourier coefficients. The sparse matrix that results is usually Huffman encoded. Although LZW and Arithmetic codes generally provide more compression than Huffman, the latter tends to work better on sparse data files that contain a large number of common values.

A 2-D Fourier Transform is required for image files since images are 2-D. The 2-D transform is equivalent to taking the 1-D transform of each row, and then taking the 1-D transform of each column, for an image matrix. The problem with the Fourier Transform is that it leaves us with a real component and an imaginary component for each pixel in the image. By symmetrically extending the image before taking the 2-D Fourier transform, the imaginary, or sine, terms can be forced to zero.

Assume an image is represented by the $4 \times 4$ matrix, $U$, such that

$$
[U]=\left[\begin{array}{rrrr}
127 & 123 & 119 & 110 \\
115 & 11 & 103 & 98 \\
99 & 87 & 85 & 83 \\
94 & 82 & 81 & 79
\end{array}\right]
$$

The 2-D Fourier Transform will produce 32 terms, or a real term (cosine) and an imaginary term (sine) for each of the 16-pixel values in the matrix. By symmetrically extending $U$, either the cosine terms or the sine terms can be forced to be identically zero.

A symmetrical extension of $U$ given by 


$\left[\begin{array}{rrrrrrrr}0 & 0 & 0 & 0 & 0 & 0 & 0 & 0 \\ 0 & 127 & 123 & 119 & 110 & 119 & 123 & 127 \\ 0 & 115 & 111 & 103 & 98 & 103 & 111 & 115 \\ 0 & 99 & 87 & 85 & 83 & 85 & 87 & 99 \\ 0 & 94 & 82 & 81 & 79 & 81 & 82 & 94 \\ 0 & 99 & 87 & 85 & 83 & 85 & 87 & 99 \\ 0 & 115 & 111 & 103 & 98 & 103 & 111 & 115 \\ 0 & 127 & 123 & 119 & 110 & 119 & 123 & 127\end{array}\right]$

will produce only nonzero cosine coefficients under a 2-D Fourier Transform.

This modification of the Fourier Transform is called a Cosine Transform and allows the matrix $U$ to be fully represented by 16 unique coefficients, most of them close to zero. If a 2-D Fast Fourier Transform is performed on the symmetrically extended matrix, the results are equivalent to a Fast Cosine Transform. It is important to note that the coefficients produced by a Cosine Transform are not the same as the real part of the Fourier Transform applied to the original matrix, $U$.

An antisymmetric extension of $U$ given by

$$
\left[\begin{array}{rrrrrrrrr}
0 & 0 & 0 & 0 & 0 & 0 & 0 & 0 & 0 \\
0 & 127 & 123 & 119 & 110 & -127 & -123 & -119 & -110 \\
0 & 115 & 111 & 103 & 98 & -115 & -111 & -103 & -98 \\
0 & 99 & 87 & 85 & 83 & -99 & -87 & -85 & -83 \\
0 & 94 & 82 & 81 & 79 & -94 & -82 & -81 & -79 \\
0 & -127 & -123 & -119 & -110 & 127 & 123 & 119 & 110 \\
0 & -115 & -111 & -103 & -98 & 115 & 111 & 103 & 98 \\
0 & -99 & -87 & -85 & -83 & 99 & 87 & 85 & 83 \\
0 & -94 & -82 & -81 & -79 & 94 & 82 & 81 & 79
\end{array}\right]
$$

will produce only nonzero sine coefficients under a 2-D Fourier Transform. This modification of the Fourier Transform is called a Sine Transform.

\section{Representation of 8-Bit Images}

The images used in this article are raw 8-bit grayscale images. The word "raw" is used to enforce the idea that there is no header on the file. The test image used for lossy compression, DBSJ.4, contains 448 columns and 280 rows for a total of 125,540 bytes. Each byte controls the grayscale intensity of a pixel on the screen with 0 representing black and 255 representing white, for a total of 256 shades of gray. A separate palette file controls whether the image is grayscale or color. The palette files used with the program "Image" consist of 768 bytes, or 256 bytes for the color red, 256 bytes for green, and 256 bytes for blue. If a grayscale image is desired, then the palette "gray.pal" is used and includes identical 256-byte segments for red, green, and blue. The actual computation of the color of each pixel in an RGB image can be seen easily in line 380 . For 
color images, bits 5,6 , and 7 determine the intensity of the red component; bits 2, 3, and 4 define the green intensity; and bits 0 and 1 provide four levels of blue. The palette file defines the mapping of the red, green, and blue bits to various intensity levels. VGA (Video graphic adapter) mode 13 is set up in line 220 for $320 \times 200$ resolution with 256 colors, or shades of gray, depending on the palette that is selected. The program "Image" is useful for displaying an image prior to compression, and then showing the degradation caused by a lossy compression/decompression routine.

While the program "Image" can let the user visually determine the quality of a reconstructed image by comparison to the original, the program "MSE" computes the mean-square-error between the original image and the reconstructed image to provide an analytical measure of quality. The mean-square-error is defined as

$$
M S E=\frac{1}{N} \sum_{i=1}^{N}\left(X_{i}-X_{i}^{\prime}\right)^{2}
$$

where $X_{i}$ is the $\mathrm{i}^{\text {th }}$ pixel in the original image, $X_{i}^{\prime}$ is the $\mathrm{i}^{\text {th }}$ pixel in the reconstructed image, and $N$ is the number of pixels. In general, a $M S E$ of five or less indicates very little loss of detail.

\section{The Discrete Cosine Transform (DCT)}

The DCT is simply a separate mathematical method for generating the Cosine Transform without explicitly using the Fourier Transform. It is based on defining a matrix $[C]$ such that

$$
\begin{aligned}
& C(k, n)=\frac{1}{\sqrt{N}} \text { for } k, n=0 \\
& C(k, n)=\sqrt{\frac{2}{N}} \cos \left[\pi \cdot(2 \cdot k+1) \cdot \frac{n}{2 \cdot N}\right] \text { otherwise }
\end{aligned}
$$

If an image is divided into blocks of size $8 \times 8$, then the matrix $C$ must also be $8 \times 8$ and becomes 


$$
[C]=\left[\begin{array}{rrrrrrrr}
0.35355 & 0.35355 & 0.35355 & 0.35355 & 0.35355 & 0.35355 & 0.35355 & 0.35355 \\
0.49039 & 0.41573 & 0.27778 & 0.0975 & -0.0975 & -0.27778 & -0.41573 & -0.49039 \\
0.46193 & 0.19134 & -0.19134 & -0.46193 & -0.46194 & -0.19134 & 0.19133 & 0.46193 \\
0.41573 & -0.09754 & -0.49039 & -0.27778 & 0.27778 & 0.49039 & 0.09754 & -0.41573 \\
0.35355 & -0.35355 & -0.35355 & 0.35355 & 0.35355 & -0.35355 & -0.35355 & 0.35355 \\
0.27778 & -0.49039 & 0.09754 & 0.41573 & -0.41573 & -0.09754 & 0.49039 & -0.27778 \\
0.19134 & -0.46194 & 0.46193 & -0.19133 & -0.19134 & 0.46194 & -0.46193 & 0.19133 \\
0.09754 & -0.27778 & 0.41573 & -0.49039 & 0.49039 & -0.41573 & 0.27777 & -0.09754
\end{array}\right]
$$

If a matrix $U$ represents the pixels in an $8 \times 8$ block of an image, then the DCT is define by the equation

$$
[V]=[C][U]\left[C^{T}\right]
$$

using matrix multiplication and $\left[C^{T}\right]$ as the transpose of matrix $[C] .[V]$ becomes a sparse matrix with few large coefficients. For example,

$$
\text { let }[U]=\left[\begin{array}{llllllll}
48 & 48 & 51 & 53 & 54 & 54 & 57 & 62 \\
50 & 51 & 52 & 52 & 57 & 59 & 59 & 63 \\
51 & 52 & 55 & 56 & 59 & 61 & 62 & 67 \\
53 & 55 & 57 & 57 & 62 & 64 & 63 & 66 \\
56 & 58 & 57 & 60 & 65 & 67 & 68 & 68 \\
56 & 59 & 61 & 63 & 66 & 66 & 70 & 72 \\
60 & 61 & 64 & 65 & 67 & 70 & 72 & 72 \\
62 & 62 & 65 & 70 & 69 & 70 & 73 & 76
\end{array}\right]
$$

which represents the first $8 \times 8$ subblock in the grayscale test file DBSJ. 4 . The actual transform file, $V$, is rounded to integer values and becomes

$$
[V]=\left[\begin{array}{rrrrrrrr}
61 & -5 & 0 & 0 & 0 & 0 & 0 & 0 \\
-5 & 0 & 0 & 0 & 0 & 0 & 0 & 0 \\
0 & 0 & 0 & 0 & 0 & 0 & 0 & 0 \\
0 & 0 & 0 & 0 & 0 & 0 & 0 & 0 \\
0 & 0 & 0 & 0 & 0 & 0 & 0 & 0 \\
0 & 0 & 0 & 0 & 0 & 0 & 0 & 0 \\
0 & 0 & 0 & 0 & 0 & 0 & 0 & 0 \\
0 & 0 & 0 & 0 & 0 & 0 & 0 & 0
\end{array}\right]
$$

which contains a large number of zeroes, but retains almost all of the information in the original subimage $[U]$. Entropy analysis of the original matrix $[U]$ reveals that a compression of 1.77 to 1 is possible using Huffman encoding (as shown by running ENTROPY.EXE). However, a compression of 25.6 to 1 is possible using Huffman encoding of the matrix [V]. This method is the basis of the Joint Photographic Expert Group (JPEG) algorithm in which images are divided into $8 \times 8$ subblocks; each subblock is processed independently by a DCT algorithm, and then the resulting file is Huffman encoded. 
The inverse DCT can be used to regenerate an approximation to the matrix $[U]$ using the equation

$$
\left[U^{\prime}\right]=\left[C^{T}\right][V][C]
$$

where $\left[U^{\prime}\right]$ represents the reconstructed image. Using the inverse DCT, the matrix $\left[U^{\prime}\right]$ becomes

$$
\left[U^{\prime}\right]=\left[\begin{array}{llllllll}
47 & 48 & 50 & 53 & 55 & 58 & 60 & 61 \\
48 & 49 & 51 & 54 & 57 & 59 & 61 & 62 \\
50 & 51 & 53 & 56 & 58 & 61 & 63 & 64 \\
53 & 54 & 56 & 58 & 61 & 64 & 65 & 67 \\
55 & 57 & 58 & 61 & 64 & 66 & 68 & 69 \\
58 & 59 & 61 & 64 & 66 & 69 & 71 & 72 \\
60 & 61 & 63 & 65 & 68 & 71 & 73 & 74 \\
61 & 62 & 64 & 67 & 69 & 72 & 74 & 75
\end{array}\right]
$$

which is nearly identical to the original image $[U]$ and differs by a meansquare-error of 1.85 indicating that visual differences will not be perceptible. A compression scheme using the DCT can be summarized in the following steps:

a. Divide the image into $8 \times 8$ blocks.

b. Perform the DCT on each $8 \times 8$ block.

c. Replace values close to zero with zero.

d. Huffman encode the resulting file.

Decompression involves the following:

a. Huffman decode the compressed file.

b. Perform the inverse DCT on each $8 \times 8$ block.

The entire DBSJ.4 image is shown in Figure 3 before and after compression at a rate of 25:1. The programs DCT.BAS and INVDCT.BAS were used for the transformations. The final compression of 25:1 was achieved by truncating any DCT coefficients less than five to zero and then entropy encoding of the remaining DCT coefficients.

The JPEG algorithm is basically an $8 \times 8$ DCT with some enhancements. JPEG is generally considered feasible for compression of still images in the 10:1 to 25:1 range. Another standard, Motion Picture Expert Group (MPEG), is used for motion video and exploits the images differences frame-by-frame. MPEG is considered feasible for the compression of realtime video images at the rate of 50:1. 

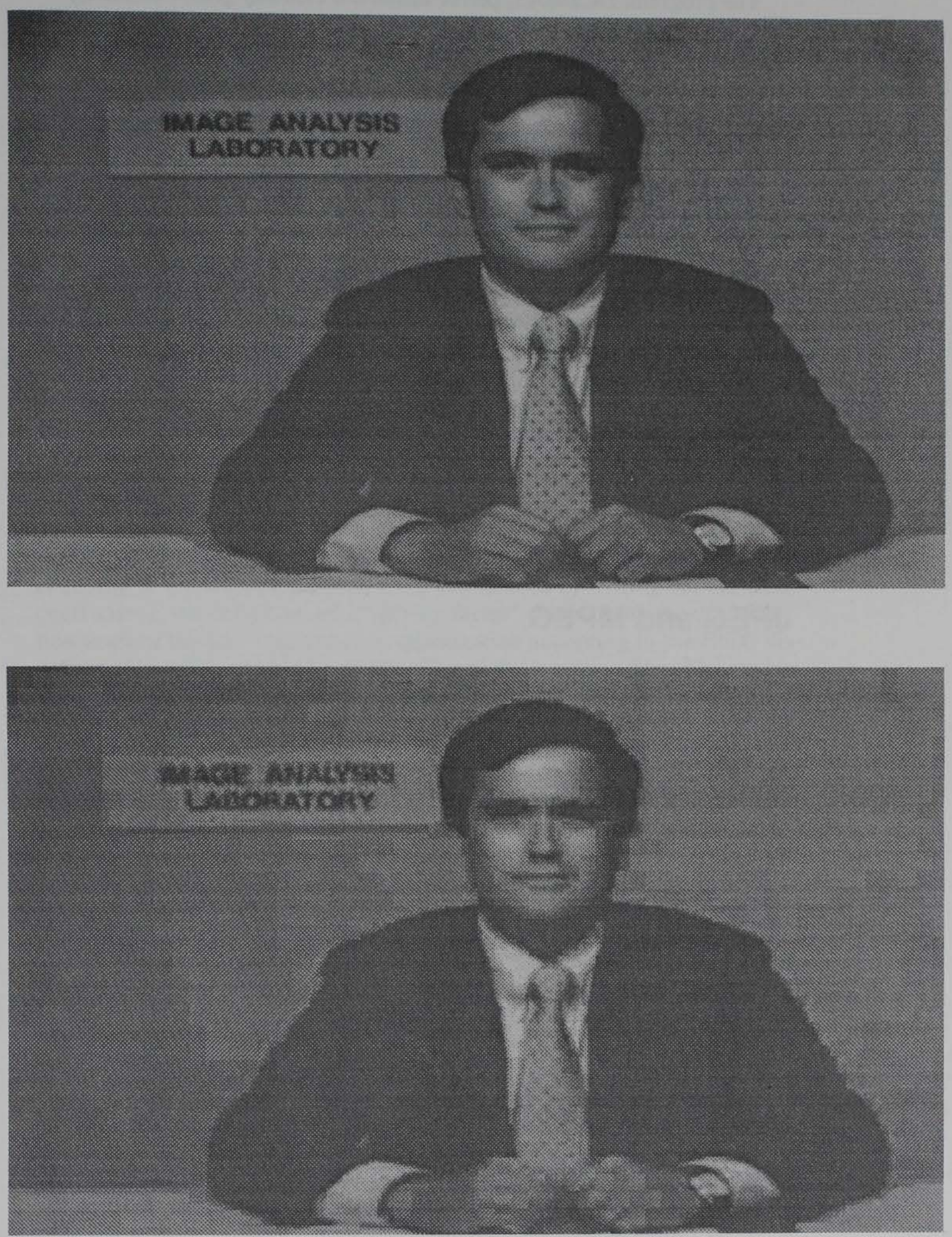

Figure 3. These photographs show the original image (top), and the restored (bottom) after 25:1 compression (MSE $=25.39$ using a threshold level of 5 in the DCT coefficient matrix) 
The program, DCT.BAS, performs the DCT on any grayscale image file. $X$ and $Y$ dimensions are required as input parameters. The output file is given the extension.DCT.

The .DCT file is a binary file. The user can perform Huffman encoding directly on this file to achieve a high level of compression. Even higher levels of compression can be obtained by defining a threshold level in the .DCT file such that coefficients below the threshold are replaced by zero before Huffman encoding.

An inverse DCT can be obtained using INVDCT.BAS. This program is very similar to DCT.BAS. The major change is that the indices of matrix $[C]$ are swapped in lines 630 and 730 . Since matrix $[C]$ is orthogonal, the transpose of $[C]$ is identical to the inverse of $[C]$. The quality of the restoration depends on the threshold level set previously for the truncation of the DCT coefficients. These two programs will also handle other types of transforms including Hadamard and Sine transforms simply by modifying the subroutine that defines the transform matrix $[C]$. The Hadamard and Sine transforms do not provide as much compression as the Cosine transform, so their discussion is presented in a study by Jain (1989).

\section{JPEG and MPEG}

While the JPEG algorithm was defined for still images and is based on the DCT, MPEG is meant primarily for motion video and obtains additional compression by exploiting interframe redundancy. Basically, if an $N x N$ pixel block changes very little in successive frames, then the DCT coefficients saved from a previous frame can be reused to represent the $N^{2}$ pixel values. The basis for many proposed HDTV systems is MPEG.

The JPEG algorithm processes 8-bit color RGB (red, green, blue) images by first translating from RGB to $Y-C_{b}-C_{r}$. For example, an original $512 \times 512$ eight-bit RGB color image contains 262,144 bytes. The three most significant bits define the $R E D$ intensity. The next three bits define the GREEN intensity, and the lowest two bits determine the BLUE intensity. In preparation for $Y-C_{b}-C_{r}$ conversion, three temporary $512 \times 512$ files are created including a $512 \times 512$ file for the $R E D$ component which consists of the three most significant bits (MSBs) corresponding to red padded with five zeroes, a $512 \times 512$ file for the GREEN component which consists of the three bits for green moved to the most significant position and padded with five zeroes, and a $512 \times 512$ file for the $B L U E$ component which consists of the two bits for blue moved to the most significant position and padded with six zeroes. The translation format from RGB to $Y$ $C_{b}-C_{r}$ is shown as 


$$
\begin{gathered}
Y=0.299 \cdot R E D+0.587 \cdot G R E E N+0.114 \cdot B L U E \\
C_{b}=-0.16874 \cdot R E D-0.33126 \cdot G R E E N+0.5 \cdot B L U E \\
C_{r}=0.5 \cdot R E D-0.41869 \cdot \text { GREEN }-0.08131 \cdot \text { BLUE }
\end{gathered}
$$

Each of the resulting $Y, C_{b}$, and $C_{r}$ files are also $512 \times 512$ eight-bit files. The $Y$ component (luminance) contains the majority of the information while the bandwidths of the $C_{b}$ and $C_{r}$ components are relatively small by comparison and can therefore be compressed at a much higher ratio without loss of information. A DCT can now be done separately on the $Y, C_{b}$, and $C_{r}$ files. RGB format is regained with the equations

$$
\begin{aligned}
R E D & =Y+1.402 \cdot C_{r} \\
\text { GREEN } & =Y-0.34414 \cdot C_{b}-0.71414 \cdot C_{r} \\
\text { BLUE } & =Y+1.772 \cdot C_{b}
\end{aligned}
$$

The JPEG algorithm is actually a modified DCT that includes DCPM encoding of the DC coefficients, a zig-zag method of writing out the DCT coefficients, the definition of a "quality factor" that defines the quantization level of the DCT coefficients, quantization according to the JPEG visualization matrix, and Huffman encoding of the resulting file. The reader is referred to Nelson (1991) for complete details on JPEG.

\section{Singular Value Decomposition (SVD)}

Singular Value Decomposition (SVD) is useful mainly as a gauge against which to measure the performance of other transform techniques and in image restoration. The SVD concentrates the maximum amount of energy in the fewest eigenvalues and is optimal in the least square sense.

Let $[U]$ be an image matrix. The matrices $[U]\left[U^{T}\right]$ and $\left[U^{T}\right][U]$ are nonnegative and symmetric and have the identical eigenvalues, $\left\{\lambda_{m}\right\}$. Assuming that $U$ is an $N x N$ matrix of $N^{2}$ pixels, there are at most $r \geq N$ nonzero eigenvalues. It is possible to find $r$ orthogonal $N x 1$ eigenvectors $\left\{\Phi_{m}\right\}$ of $\left[U^{T}\right][U]$, and $r$ orthogonal $N x 1$ eigenvectors $\left\{\Psi_{m}\right\}$ of $[U]\left[U^{T}\right]$, that is

$$
\begin{aligned}
& {\left[U^{T}\right][U] \Phi_{m}=\lambda_{m} \Phi_{m}, m=1, \ldots \ldots, r} \\
& {[U]\left[U^{T}\right] \Psi_{m}=\lambda_{m} \Psi_{m}, m=1, \ldots \ldots, r}
\end{aligned}
$$

The matrix $U$ has the representation 


$$
[U]=\Psi \Lambda^{0.5} \Phi^{T}=\sum_{m=1}^{r} \Psi_{m} \Phi_{m}^{T} \sqrt{\lambda_{m}}
$$

where $\Psi$ and $\Phi$ are $N x r$ matrices whose $m^{\text {th }}$ columns are the vectors $\Psi_{m}$ and $\Phi_{m}$, respectively, and $\Lambda^{0.5}$ is an r $x r$ diagonal matrix, defined as

$$
\Lambda^{0.5}=\left[\begin{array}{llll}
\sqrt{\Gamma_{1}} & & & \\
& \cdot & \\
& \cdot & \\
& & \cdot & \\
& & \sqrt{\Gamma_{m}}
\end{array}\right]
$$

Equation 8 is called the spectral representation, the outer product expansion, or the singular value decomposition (SVD) of $[U]$. The nonzero eigenvalues of $\left[U^{T}\right][U], \Gamma_{m}$, are also called the singular values of $[U]$. If $r \ll<$, then the image containing $N^{2}$ samples can be represented by $(N+N) r$ samples of the vectors $\left\{\lambda_{m}^{1 / 4} \Psi_{m}, \lambda_{m}^{1 / 4} \Phi_{m} ; m=1, \ldots \ldots, r\right\}$.

Since $\Psi$ and $\Phi$ have orthogonal columns, the SVD transform of the image $U$ is defined as

$$
[U]=\Psi \Lambda^{0.5} \Phi^{T}
$$

which is a separable transform that diagonalizes the given image.

The image $\left[U_{k}\right]$ generated by the partial sum

$$
\left[U_{k}\right]=\sum_{m=1}^{k} \sqrt{\lambda_{m}} \Psi_{m} \Phi_{m}^{T}, k \leq r
$$

is the best least squares rank-k approximation of $[U]$ if the $\lambda_{m}$ are in decreasing order of magnitude. For any $k \leq r$, the least squares error

$$
\varepsilon_{k}^{2}=\sum_{m=1}^{M} \sum_{n=1}^{N}\left[u(m, n)-u_{k}(m, n)\right]^{2}, k=1,2, \ldots \ldots, r
$$

reduces to

$$
\varepsilon_{k}^{2}=\sum_{m=k+1}^{r} \Gamma_{m}
$$

These equations show that the energy concentrated in the transform coefficients is maximized by the SVD transform for the given image. As an example, 


$$
\text { Let }[U]=\left[\begin{array}{ll}
1 & 2 \\
2 & 1 \\
1 & 3
\end{array}\right]
$$

The eigenvalues of $\left[U^{T}\right][U]$ are found to be $\lambda_{1}=18.06$ and $\lambda_{2}=1.94$, which give $r=2$, and the SVD transform of $[U]$ is

$$
\Lambda^{0.5}=\left[\begin{array}{cc}
4.25 & 0 \\
0 & 1.39
\end{array}\right]
$$

The eigenvectors are found to be

$$
\Phi_{1}=\left[\begin{array}{l}
0.5019 \\
0.8649
\end{array}\right] \quad \Phi_{2}=\left[\begin{array}{r}
0.8649 \\
-0.5019
\end{array}\right]
$$

From above, $\Psi_{1}$ is obtained via

$$
\Psi_{m}=\frac{1}{\sqrt{\lambda_{m}}}[U] \Phi_{m}
$$

to yield

$$
\left[U_{1}\right]=\sqrt{\lambda_{1}} \Psi_{1} \Phi_{1}^{T}=\left[\begin{array}{ll}
1.120 & 1.94 \\
0.953 & 1.62 \\
1.549 & 2.70
\end{array}\right]
$$

as the best least squares rank-1 approximation of $[U]$. The energy concentrated in the $K$ samples of SVD is greater than the energy concentrated in any $K$ samples of the other transform methods.

Example: Let [U] be an $N x N$ images matrix. It is desired to use SVD to achieve a $N: r$ compression using only $r$ of the largest eigenvalues. The $[U]$ matrix can then be approximated as

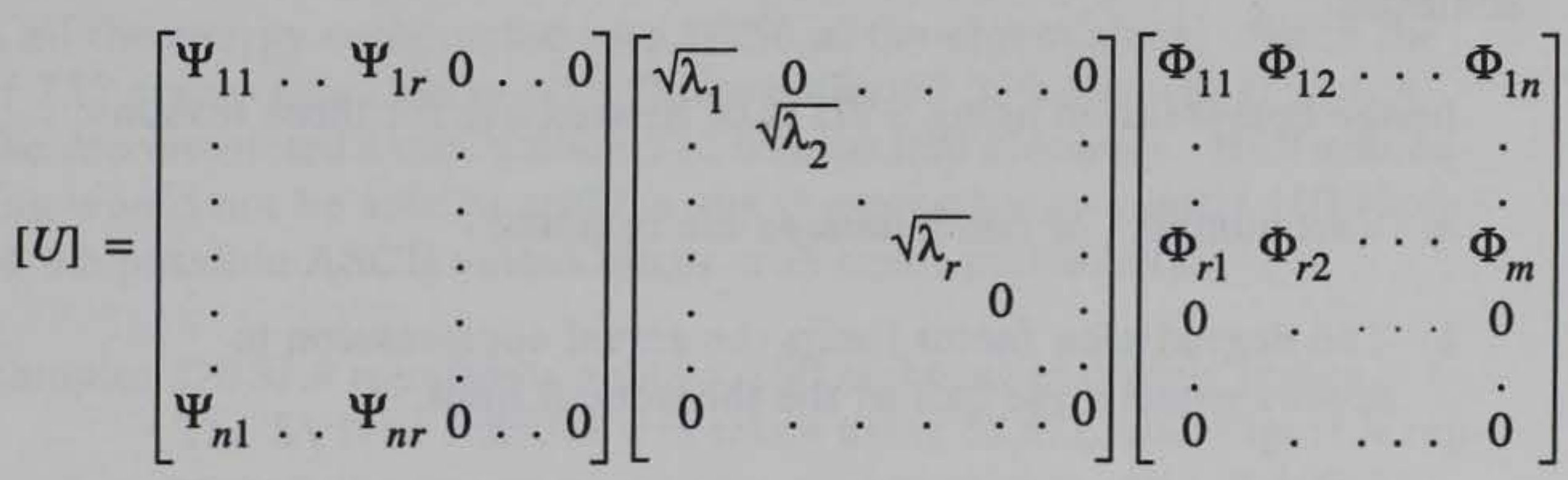

The total number of coefficients that must be encoded and transmitted are $r x N$ coefficients from matrix $\Psi$ plus $r x N$ coefficients from matrix $\Phi$ or $2 x r x N$ coefficients total, which only achieves half of the desired compression. A small improvement can be obtained by recognizing that the columns 
in $\Psi$ and the rows in $\Phi$ are orthogonal. If $\Psi$ has $r$ orthogonal columns such that

$$
\Psi=\left[\begin{array}{ccccc}
\Psi_{11} & \Psi_{12} & \Psi_{1 r} & 0 & 0 \\
\cdot & & & \cdot & \cdot \\
\cdot & & \cdot & \cdot \\
\cdot & & \cdot & \cdot \\
\Psi_{n 1} & \Psi_{n 2} & \Psi_{n r} & 0 & 0
\end{array}\right]
$$

with $r x N$ nonzero elements, then the $r N$ elements of $\Psi$ can be reconstructed using the conditions for orthogonality from the matrix $\Psi^{\prime}$ with

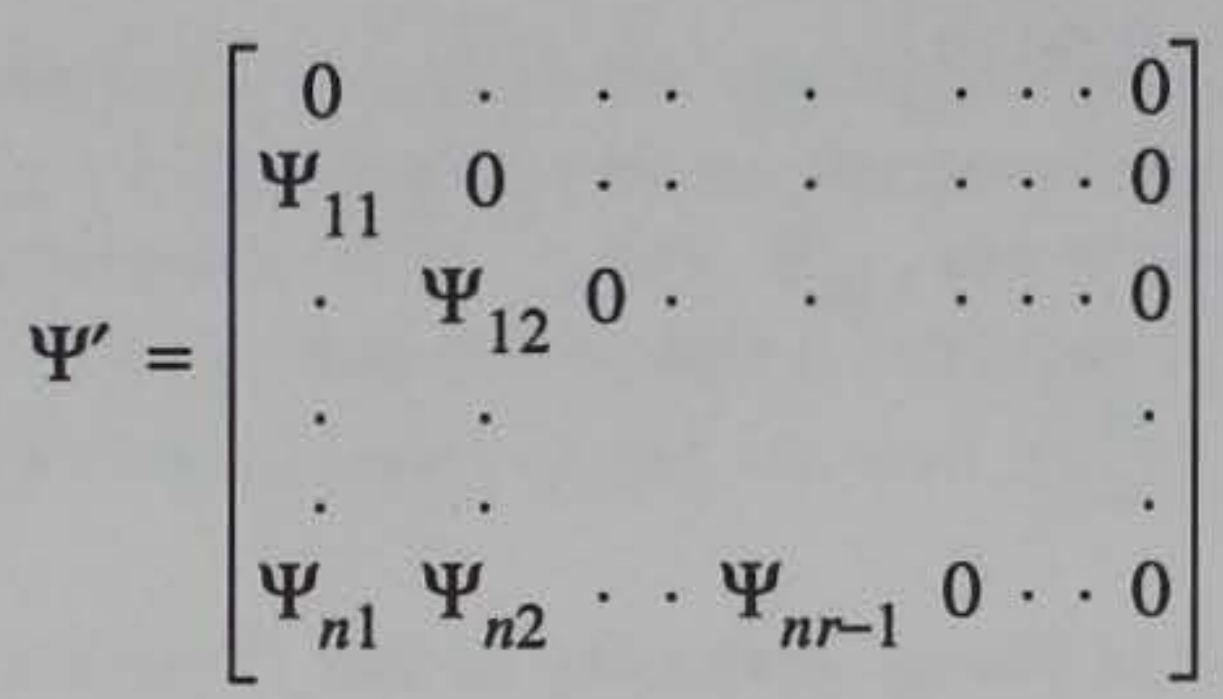

where $\Psi^{\prime}$ has $r N-1 / 2 r^{2}$ nonzero elements. The number of elements that are required to be transmitted are $r x N-1 / 2 r_{2}$ for $\Psi$ and $r x N-1 / 2 r 2$ for $\Phi$ or $r(2 N-r)$ coefficients total, as opposed to $\mathrm{r} x N$. Therefore, a degradation factor, $D$, can be computed as

$$
D=2-r^{\prime}
$$

where $r$ ' equals r'/N and r' represents the percent compression. Therefore, if an attempt is made to achieve 90 percent compression with SVD on a data file of size $N^{2}$ by using only $1 / 10^{\text {th }}$ of the eigenvalues, the actual size of the data file that has to be transmitted is $0.1 \times(2-0.1) \times N^{2}$, or 19 percent of the original size, so that a compression of only 81 percent is achieved.

Image compression using SVD is of limited use for three reasons:

a. Vast numbers of computations are required.

$b$. The degradation factor limits the actual compression to approximately one half of the theoretical limit.

c. Each coefficient in the $\Psi$ and $\Phi$ matrices will require more that a single byte of accuracy.

Example: If the SVD transform of an image matrix, $[U]$, produces eigenvalues that are zero, then by not transmitting these zero eigenvalues and their corresponding zero rows and columns in $\Psi$ and $\Phi^{T}$, respectively, lossless image 
compression can be achieved. If $[U]$ is an $256 \times 256$ matrix given by

$$
[U]=\left[\begin{array}{c}
0,1,2,3,4,5, \ldots \ldots \ldots, 255 \\
0,1,2,3,4,5, \ldots \ldots \ldots, 255 \\
0,1,2,3,4,5, \ldots \ldots \ldots, 255 \\
\cdot \\
\cdot \\
\cdot \\
0,1,2,3,4,5, \ldots \ldots \ldots, 255
\end{array}\right]
$$

then the SVD transform produces 255 eigenvalues that are zero and one eigenvalue that is nonzero. The (unnormalized) $\Psi$ and $\Phi^{T}$ matrices become

$$
\Psi^{T}=[1,1,1,1, \ldots \ldots \ldots 1]
$$

and

$$
\Phi=[0,1,2,3,4,5, \ldots, 255]
$$

The original $U$ matrix can be reconstructed by simple matrix multiplication of $\Psi$ and $\Phi^{T}$.

All of the energy is compacted into a single eigenvalue for a theoretical possible compression of $1 / 256$, but notice that a total of 512 bytes must be transmitted in order to send the 256-element $\Psi$ matrix and the 256element $\Phi$ matrix. Therefore, only a compression of $1 / 128$ can be realized which is consistent with the degradation factor

$$
D=\frac{1}{256} \cdot\left(2-\frac{1}{256}\right) \approx 2
$$

with all the energy compressed into $1 / 256$ of the eigenvalues. Since the other 255 eigenvalues are exactly 0 , the entire 65,536-element $U$ matrix can be reconstructed exactly from 512 transmitted elements. Huffman encoding would not be able to achieve any compression on matrix $[U]$ since all of the possible ASCII values occur with equal probability.

Example: DBSJ.4 represents a $448 \times 280$ eight-bit grayscale image. The SVD of DBSJ.4 was taken using MATLAB. Figure 4 represents the image quality for a compression ratio of 100:1. 


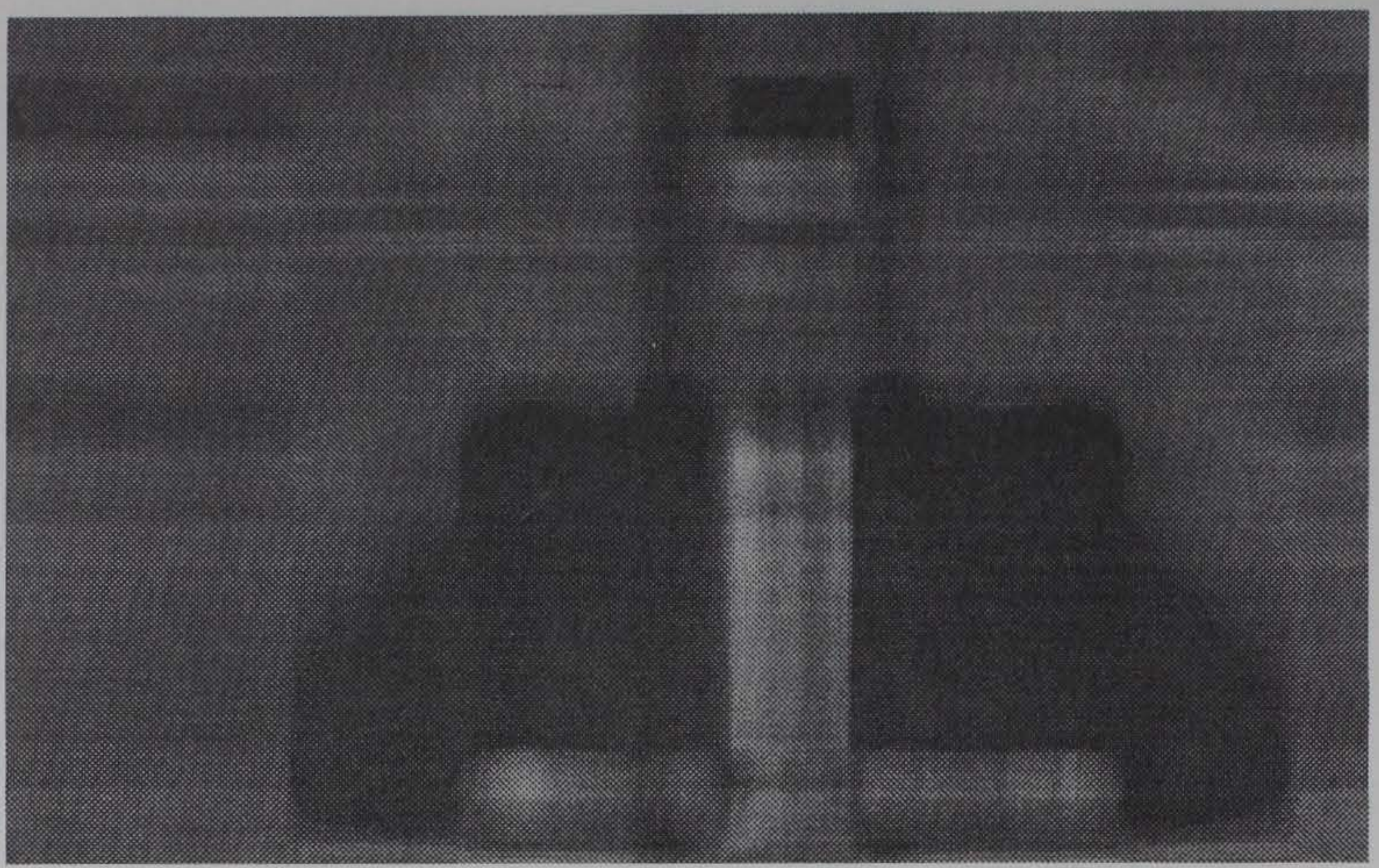

Figure 4. DBSJ.4 images compressed with SVD at 100:1 with a mean-square error of 553

\section{Fractal Compression}

Fractal compression is a new technique that promises compression ratios of 500:1 without noticeable loss in image quality. It is based on taking a portion of the image and reproducing the rest of the image with a set of affine transformations that consist of translations, rotations, and scaling. A set of iterated function system (IFS) codes defines the image. Once the IFS codes for an image have been determined, then a simple iterated procedure is used to regenerate the image.

The iterated function is defined by the IFS codes. Essentially, $X$ and $Y$ coordinates of the new pixel are computed from $X$ and $Y$ coordinates of the previous pixel. The IFS codes given below will illustrate the procedure. The program, IFS.BAS, will generate a solid rectangle from these IFS codes in a random and rather spectacular way. 


\section{IFS Codes for a Square}

\begin{tabular}{|l|l|l|l|l|l|l|}
\hline A & B & C & D & E & F & P \\
\hline 0.5 & 0 & 0 & 0.5 & 1 & 1 & 0.25 \\
\hline 0.5 & 0 & 0 & 0.5 & 50 & 1 & 0.25 \\
\hline 0.5 & 0 & 0 & 0.5 & 1 & 50 & 0.25 \\
\hline 0.5 & 0 & 0 & 0.5 & 50 & 50 & 0.25 \\
\hline
\end{tabular}

From the IFS codes, new values of $X$ and $Y$ are computed from one of four sets of equations as follows:

a. Equation Set 1: New $X=A X+B Y+1$

$$
\text { New } Y=C X+D Y+1
$$

b. Equation Set 2: New $X=A X+B Y+50$

$$
\text { New } Y=C X+D Y+1
$$

c. Equation Set 3: New $X=A X+B Y+1$

New $Y=C X+D Y+50$

d. Equation Set 4: New $X=A X+B Y+50$

New $Y=C X+D Y+50$

The variable $P$ indicates that the probability that any one set is used to compute the new $X$ and $Y$ coordinates is 0.25 . After each iteration of one set of equations, the $X$ and $Y$ coordinates are plotted, and another set of equations is randomly chosen to compute the next point.

The procedure is illustrated graphically in Figure 5. The $X$ and $Y$ coordinates are scaled by 0.5 and either a 1 or 50 is added to the $X$ and/or $Y$ values. This procedure is repeated until the entire rectangle is painted.

Any image can be reduced to sets of IFS codes. Barnsley (1988) provides other IFS codes that can be used with IFS.BAS for more exotic images.

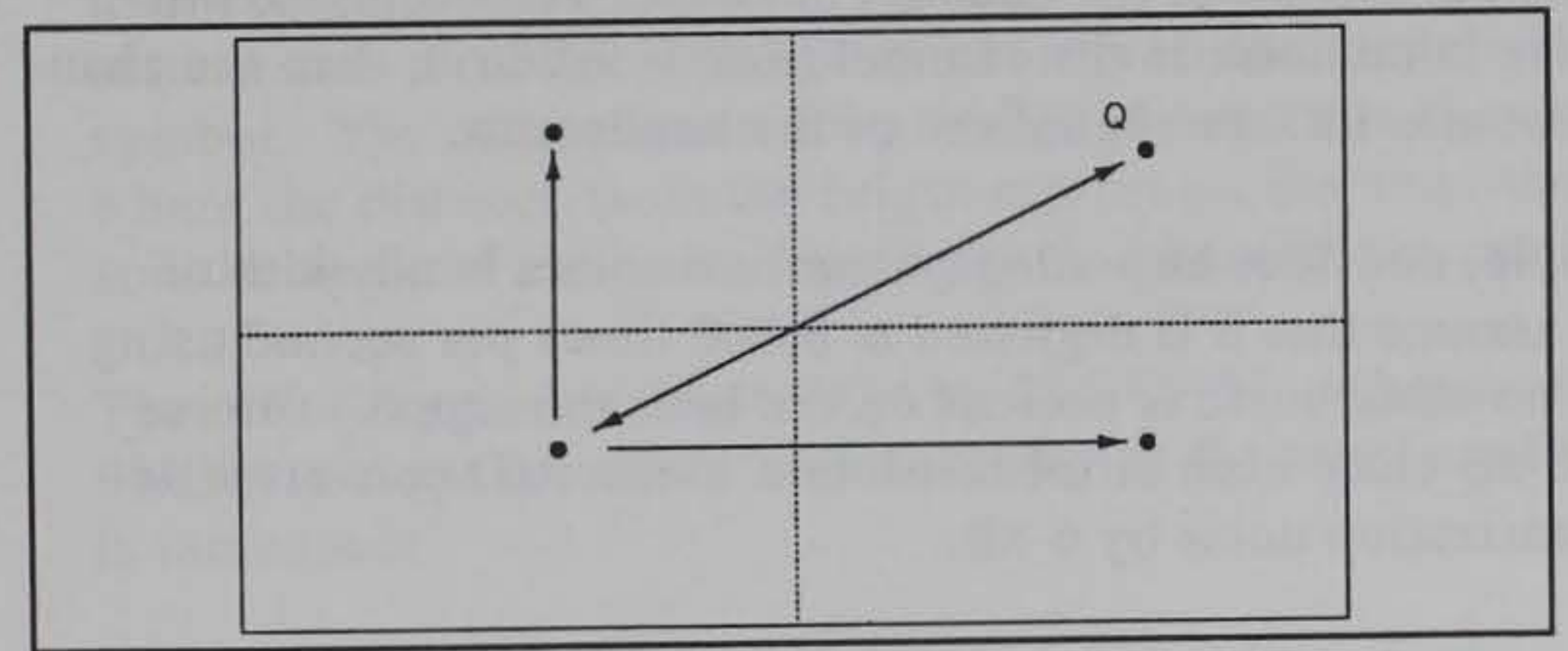

Figure 5. Random mapping of point $Q$ for a fractal process 


\section{Image Compression and Bandwidth Reduction}

\section{Communication Standards}

An analog NTSC cable TV system requires $6 \mathrm{MHz}$ per channel. It might seem that a compression of $50: 1$ would reduce the bandwidth by a factor of 50 (to $120,000 \mathrm{~Hz}$ ), but it does not. Using an estimated resolution of $512 \times 480$ for TV with 8-bit color and a frame rate of 30 times per second, the required bit rate for digital transmission is $58,982,000$ bits/ sec, or approximately $60 \mathrm{Mbps}$. Since compression techniques are inherently digital, the $6-\mathrm{MHz}$ analog signal was converted to a $60,000,000$-bit/ sec digital signal and now requires an absolute minimum bandwidth of $30 \mathrm{MHz}$ (based on $1 \mathrm{bit} / \mathrm{symbol}$ ). Compression by $50: 1$ would result in a total transmission bandwidth of at least $600,000 \mathrm{KHz}$.

Shannon's law dictates the maximum transmission speed of digital data. This speed limit is determined by only two factors, which include the bandwidth of the transmission channel and the signal-to-noise ratio of the channel. In a digital system, the maximum data rate, called the channel capacity $C$, is bounded by Shannon's law which is given by

$$
C \text { bit/sec }=B W \log _{2}\left(1+\frac{S}{N}\right)
$$

where $B W$ is the bandwidth of the channel in Hertz. According to this equation, if there is no noise in the channel ( $S / N=$ infinity), then the channel capacity becomes infinite regardless of the bandwidth.

As an example, consider an analog phone line with a bandwidth of $4,000 \mathrm{~Hz}$, and assume that it is digitized at 8,000 times per second using $8 \mathrm{bits} / \mathrm{byte}$. If no other noise is present on the line, the signal-to-noise ratio will be $48 \mathrm{~dB}$ since each bit of resolution in the A/D convertor decreases the quantization noise by $6 \mathrm{~dB}$.

To use Shannon's law, the S/N ratio must be expressed in linear terms, or $\mathrm{S} / \mathrm{N}=10^{(48 / 10)}$. The channel capacity then becomes 


$$
C=64,000 \text { bits } / \mathrm{sec}=4,000 \log _{2}\left(1+10^{4.8}\right)
$$

and will not be affected by the number of bits encoded in a symbol. The theoretical limit essentially states that it is possible to build dial-up modems that transmit without error at rates up to $64,000 \mathrm{bits} / \mathrm{sec}$. Shannon's law does not tell us how to design hardware to achieve the theoretical maximum bit rate. The common quadrature phase shift keying (QPSK) and quadrature amplitude modulation (QAM) techniques used for modems have inherent deficiencies that will not allow them to obtain the maximum theoretical bit rate; however, Shannon's law can be used to determine the theoretical limit on the channel capacity.

The distinction between bit rate and symbol rate is vital in determining bandwidth requirements. The QPSK modulation technique encodes two bits in a symbol since there are four possible phases $(0,90,180$, and $270 \mathrm{deg}$ ) of the carrier. A change of phase of the carrier represents that transmission of a single symbol (baud) which conveys two bits of information according to Table 3 :

\begin{tabular}{|l|l|}
\hline \multicolumn{2}{|l|}{$\begin{array}{l}\text { Table 3 } \\
\text { QPSK Modulation }\end{array}$} \\
\hline Differential Phase & Dibits \\
\hline $0^{\circ}$ & 00 \\
\hline $90^{\circ}$ & 01 \\
\hline $180^{\circ}$ & 11 \\
\hline $270^{\circ}$ & 10 \\
\hline
\end{tabular}

Although it is common to speak of a 1,200 baud modem, the bit rate is $1,200 \mathrm{bits} / \mathrm{sec}$ and the baud rate (with QPSK modulation) is really 600 baud.

The QAM varies both the amplitude and phase of the carrier in order to encode bits into symbols. The term 16-QAM means the carrier can assume one of 16 possible states in order to encode $\log _{2}(16)$, or 4 bits/ symbol. The constellation for a 16-QAM carrier is shown in Figure 6 where the distance from the origin represents the amplitude of the carrier, and the angle of the point represents the phase of the carrier.

The difficulty with using 64-QAM, 256-QAM, or higher is that the susceptibility to noise increases exponentially as the number of bits per symbol is increased. 


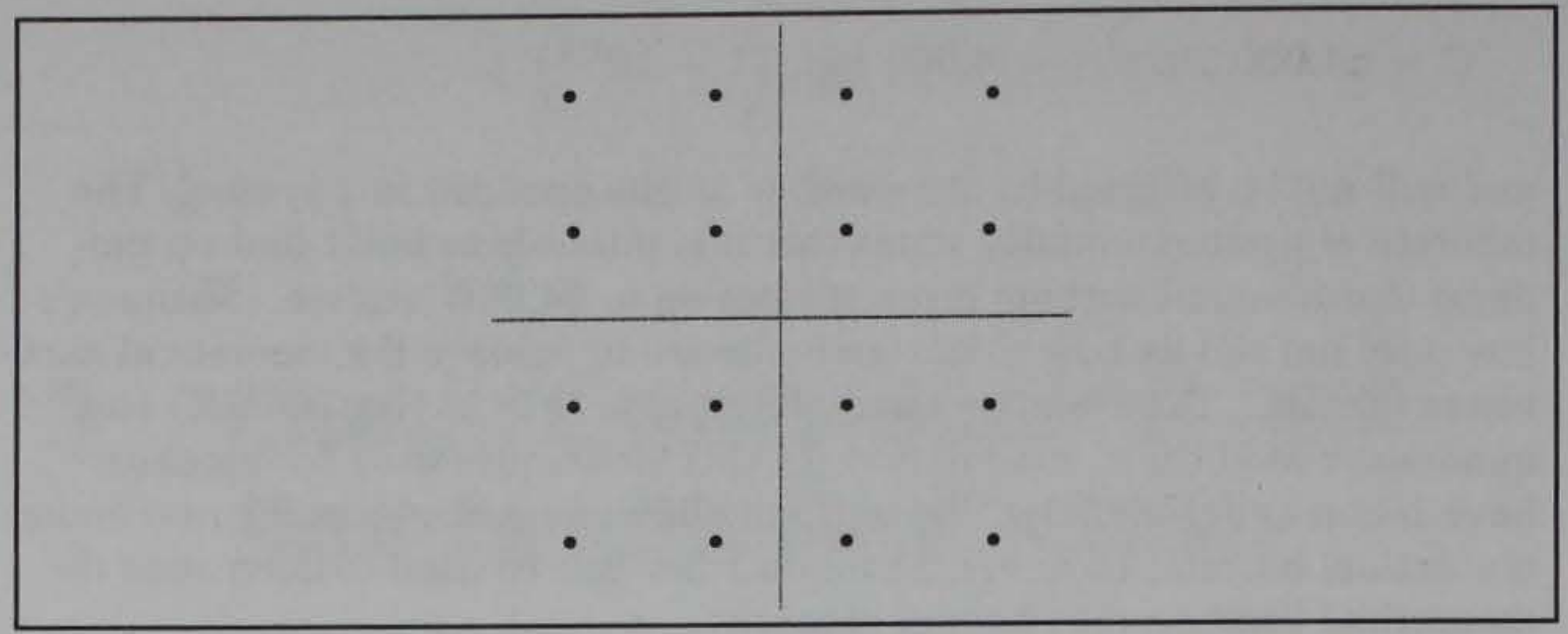

Figure 6. 16-QAM constellation

The bandwidth required for digital transmission of data is given by

$$
B W=\frac{\text { Data Rate }(\text { symbols } / \mathrm{sec}) \cdot(1+r)}{2}
$$

where $r$ represents the Nyquist rolloff factor of the raised cosine filter and $r$ falls between 0 and 1 . The basis for this equation is given by Couch (1982).

In the previous example, if the 64,000 -bit/sec data rate is to be transmitted using frequency shift keying (FSK) such that only one bit is encoded per symbol, then the symbol rate becomes 64,000 symbols $/ \mathrm{sec}$. The required transmission bandwidth is usually taken as $64,000 \mathrm{~Hz}(\mathrm{r}=1)$ in most texts, although it can approach as low as $32,000 \mathrm{~Hz}$ by using a sharp cutoff Nyquist filter $(r=0)$. Throughout the remainder of this report, it will be assumed that the required minimum transmission bandwidth is one half of the symbol rate.

Digital HDTV has a proposed resolution of $1,440 \times 960$ pixels/frame $\mathrm{x}$ $12 \mathrm{bits} /$ pixel x 30 frames/sec ( $12 \mathrm{bits} / \mathrm{pixel}=8 \mathrm{bits} / \mathrm{pixel}$ for luminance + 4 bits/pixel for chrominance at half-resolution). The data rate is about $500 \mathrm{Mbits} / \mathrm{sec}$. The aim of most HDTV systems is to broadcast at about $15 \mathrm{Mbits} / \mathrm{sec}$. This data rate requires approximately 33:1 compression. Encoding with $1 \mathrm{bit} / \mathrm{symbol}$ would require a transmission bandwidth of $7.5 \mathrm{MHz}$. Encoding with $4 \mathrm{bits} / \mathrm{symbol}$ would increase the susceptibility of the transmitted signal to noise but would only require a bandwidth of $1.875 \mathrm{MHz}$, as a minimum.

The Common Intermediate Format (CIF) is a common format for transmitting video images at 30 frames/sec over a T1 line (capacity $1.544 \mathrm{Mbits} / \mathrm{sec}$ ) or a Quarter-CIF (QCIF) picture at 10 frames/sec over Integrated Services Digital Network (ISDN) telephone line (capacity $64 \mathrm{Kbits} / \mathrm{sec}$ ).

A CIF image is composed of a luminance channel with a resolution of 288 lines/frame $x 352$ pixels/line and 8 bits/pixel, and two chrominance 
channels $\left(C_{b}\right.$ and $\left.C_{\mathrm{r}}\right)$ with half resolution, i.e., 144 lines/frame $\mathrm{x} 176$ pixels/ line and $8 \mathrm{bits} / \mathrm{pixel}$. At $30 \mathrm{frames} / \mathrm{sec}$, the data rate is $36.5 \mathrm{Mbits} / \mathrm{sec}$. Transmission over a T1 line for video conferencing applications requires a compression ratio of about 24:1. A QCIF image is one-fourth the size of a CIF image and at $10 \mathrm{frames} / \mathrm{sec}$ requires about $3 \mathrm{Mbits} / \mathrm{sec}$. Thus, video phone over ISDN network requires about 48:1 compression.

\section{Advanced Digital Television (ADTV)}

The ATDV was developed by the Advanced Television Research Consortium and uses MPEG compression to provide a data rate of $24,000,000 \mathrm{bits} / \mathrm{sec}$. It has two trellis-coded 32-QAM data carriers to provide a wide bandwidth standard priority channel and a narrower bandwidth high-priority channel, all within a 6-MHz transmission bandwidth. The high priority channel provides the viewable picture, and the additional standard-priority channel provides the full HDTV quality. The ADTV spectrum is shown in Figure 7.

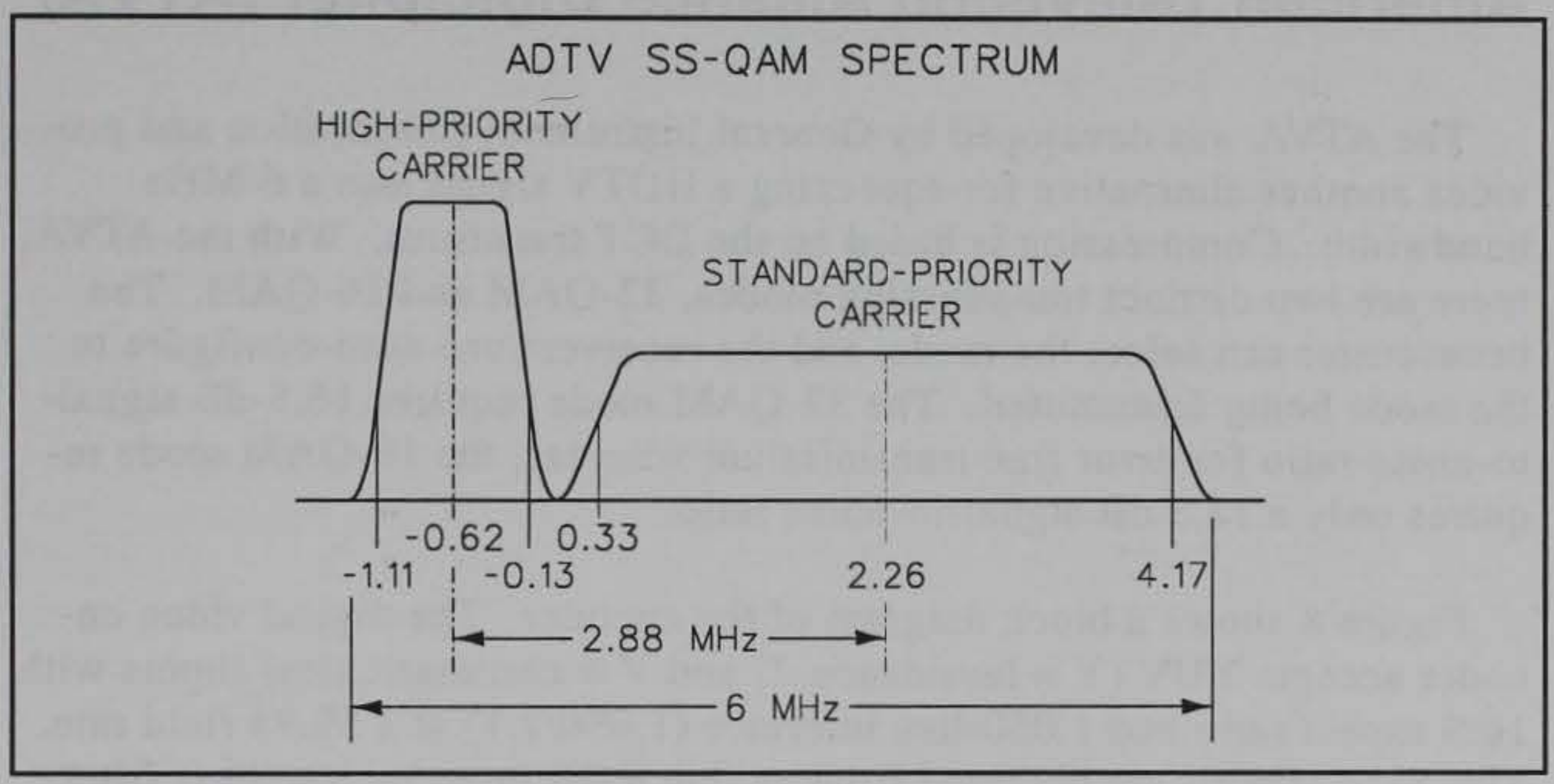

Figure 7. ATDV 32-QAM channel spectrum

The ADTV's 59.94 field rate is identical to that of the National Television Standard Committee (NTSC), thus eliminating temporal artifacts and the need for frame synchronization in mixed ADTV-NTSC environments. Its $1,440 \times 960,1,050$-line scanning format is cost-effective in the production studio. The 2:1 vertical ratio with 525-line NTSC video and 2:1 horizontal ratio with the CCIR Rec. 601 sampling standard used in the 525-line D1 tape recorders offer economical transcoding in mixed ADTV-NTSC production environments. The 16-Mbit DRAM frame memories in an ADTV receiver are predicted to cost about $\$ 13$ each by 1996. 


\section{Digital Spectrum Compatible (DSC)}

The DSC was developed by Zenith and AT\&T to provide all digital definition television simulcast by compressing the wide bandwidth digital signal into a 6-MHz channel. It uses a unique four-level vestigal sideband (4-VSB or 2 bits/symbol) modulation technique to assure noise free and interference free reception. The 4-VSB coding is complemented by a twolevel digital data system (2-VSB, or $1 \mathrm{bit} / \mathrm{symbol})$. The resulting bi-rate coding system identifies and selects the most important picture information on a scene-by-scene basis and automatically transmits that data in a two-level ( 1 bit/symbol) binary mode. The two-level digital coding makes the system far more tolerant to noise and other interference at greater distances from the transmitter.

The DSC uses a $17-\mathrm{Mbps}$ data rate and a 787.5 -line progressive scanning format to eliminate artifacts due to interlacing video.

\section{American Television Alliance Digicipher (ATVA)}

The ATVA was developed by General Instrument Corporation and provides another alternative for squeezing a HDTV signal into a $6-\mathrm{MHz}$ bandwidth. Compression is based on the DCT transform. With the ATVA, there are two distinct transmission modes, 32-QAM and 16-QAM. The broadcaster can select the mode, and the receivers can auto-configure to the mode being transmitted. The 32-QAM mode requires $16.5-\mathrm{dB}$ signalto-noise ratio for error free transmission; whereas, the 16-QAM mode requires only a $12.5 \mathrm{~dB}$ signal-to-noise ratio.

Figure 8 shows a block diagram of the encoder. The digital video encoder accepts $\mathrm{YUV}$ ( $\mathrm{Y}=$ luminance, $\mathrm{U}$ and $\mathrm{V}=$ chromaticities) inputs with $16: 9$ aspect ratio and 1,050 -line interlace $(1,050 / 2: 1)$ at a 59.94 field rate. The YUV signals are obtained from analog RGB input by low-pass filtering, A/D conversion, and an RGB-to-YUV matrix. The sampling frequency is $53.65 \mathrm{MHz}$ for $\mathrm{R}, \mathrm{G}$, and $\mathrm{B}$. The digital video encoder implements the compression algorithm and generates a video data stream.

The multiplexer combines the various data streams into one data stream at 18.22 Mbps. The forward error correction (FEC) encoder adds error correction overhead bits and provides $24.39 \mathrm{Mbps}$ of data to the 32-QAM modulation. The symbol rate of the 32-QAM signal is $4.88 \mathrm{MHz}$. 


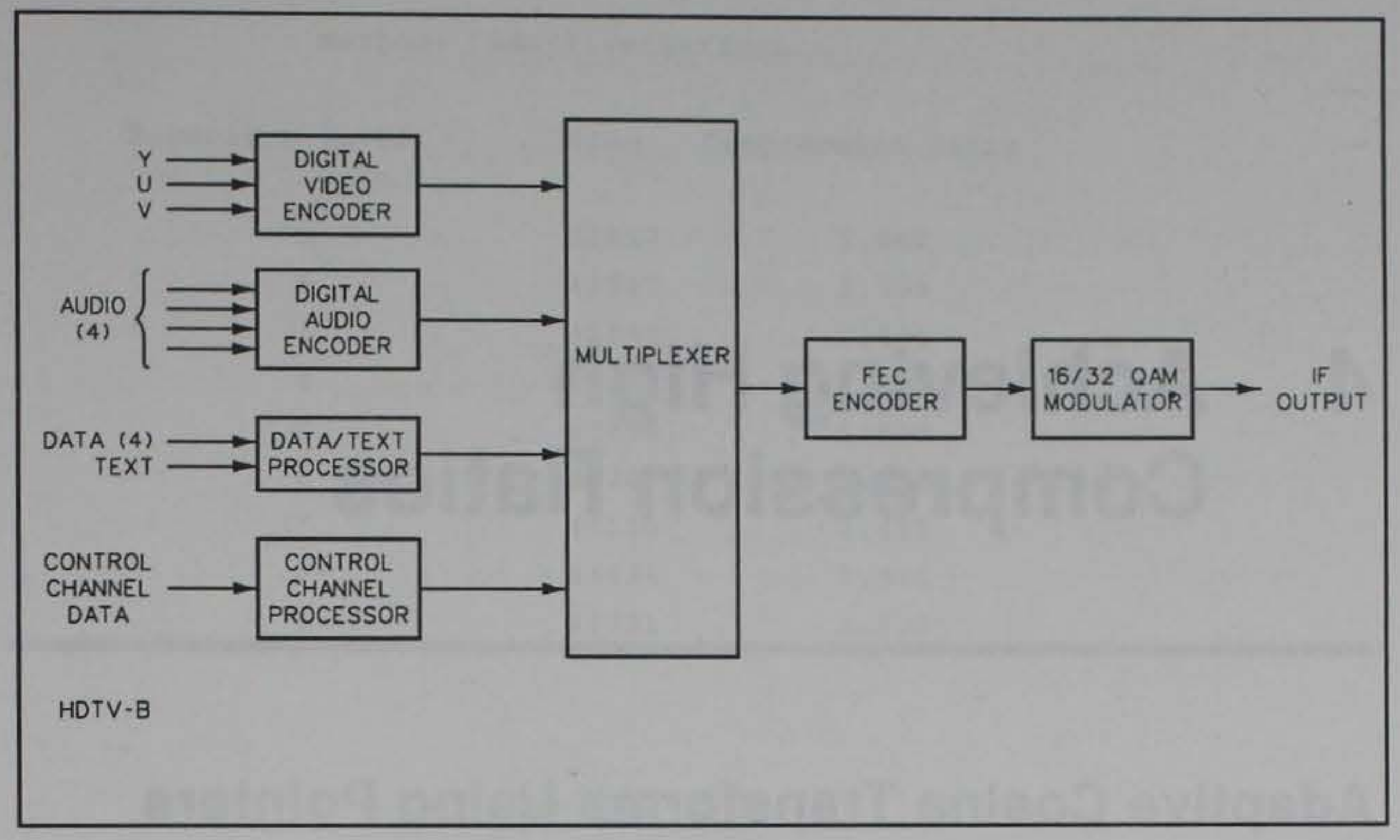

Figure 8. ATVA encoder block diagram 


\section{Achieving High Compression Ratios}

\section{Adaptive Cosine Transforms Using Pointers}

The restored image after 25:1 compression in Figure 3 is a result of a simple $8 \times 8$ DCT with thresholding and entropy encoding. Typically, Huffman encoding is not done in this report in order to produce a final compressed file; rather, the entropy is calculated according to Equation 1 to determine the maximum amount of compression that can be obtained from Huffman encoding. If the DCT matrices are sparse and contain a large number of zero entries, then Huffman encoding generally produces better results than Arithmetic, or LZW, but usually still falls short of the entropy. The results of several lossless algorithms applied recursively to different files are outlined below.

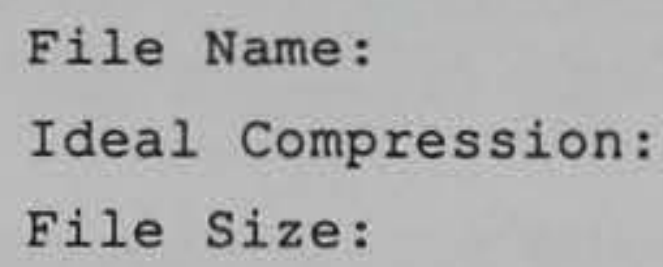

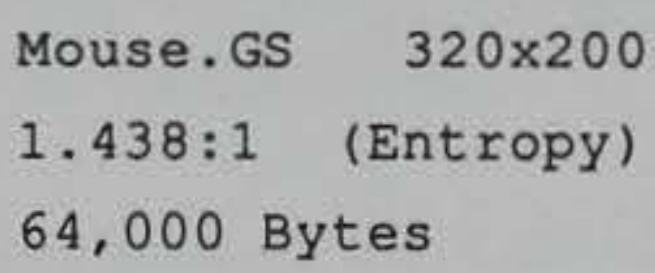


Method: Adaptive Huffman

$\begin{array}{ccc}\text { Recursion Level } & \text { Size } & \text { Compression Ratio } \\ 1 & 42617 & 1.502 \\ 2 & 42527 & 1.505 \\ 3 & 42850 & 1.494 \\ 4 & 43163 & 1.483 \\ 5 & 43476 & 1.483 \\ 6 & 43789 & 1.462 \\ 7 & 44110 & 1.451 \\ 8 & 44431 & 1.440 \\ 9 & 44751 & 1.430\end{array}$

Method: LZW

Recursion Level Size Compression Ratio

$\begin{array}{lrr}1 & 56274 & 1.137 \\ 2 & 77688 & 0.824 \\ 3 & 76959 & 0.832 \\ 4 & 89957 & 0.711 \\ 5 & 103347 & 0.619 \\ 6 & 116516 & 0.549 \\ 7 & 133250 & 0.480 \\ 8 & 151935 & 0.421 \\ 9 & 173342 & 0.369\end{array}$

Method: Arithmetic

$\begin{array}{ccc}\text { Recursion Level } & \text { Size } & \text { Compression Ratio } \\ 1 & 44846 & 1.427 \\ 2 & 45009 & 1.422 \\ 3 & 45213 & 1.416 \\ 4 & 45436 & 1.409 \\ 5 & 45658 & 1.402 \\ 6 & 45883 & 1.395 \\ 7 & 46118 & 1.388 \\ 8 & 46351 & 1.381 \\ 9 & 46585 & 1.374\end{array}$




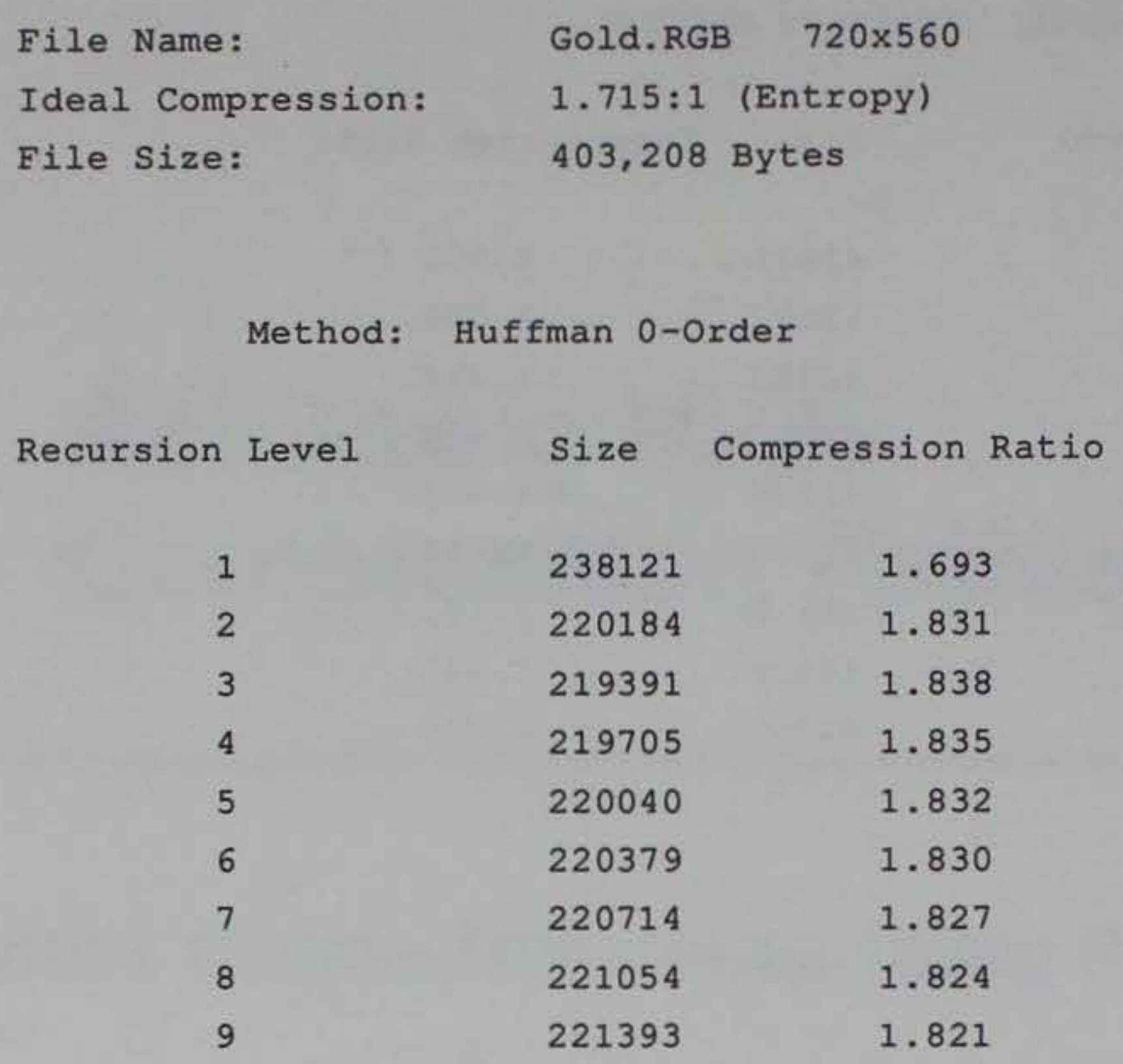

Method: Adaptive Huffman

$\begin{array}{ccc}\text { Recursion Level } & \text { Size } & \text { Compression Ratio } \\ 1 & 199988 & 2.016 \\ 2 & 186428 & 2.163 \\ 3 & 186763 & 2.159 \\ 4 & 187154 & 2.154 \\ 5 & 187535 & 2.150 \\ 6 & 187922 & 2.146 \\ 7 & 188306 & 2.141 \\ 8 & 188702 & 2.137 \\ 9 & 189104 & 2.132\end{array}$

Method: LZW

$\begin{array}{ccc}\text { Recursion Level } & \text { Size } & \text { Compression Ratio } \\ 1 & 194810 & 2.070 \\ 2 & 265701 & 1.518 \\ 3 & 276317 & 1.459 \\ 4 & 322433 & 1.251 \\ 5 & 363944 & 1.108 \\ 6 & 415781 & 0.970 \\ 7 & 471263 & 0.856 \\ 8 & 538827 & 0.748 \\ 9 & 614199 & 0.656\end{array}$


Method: Arithmetic

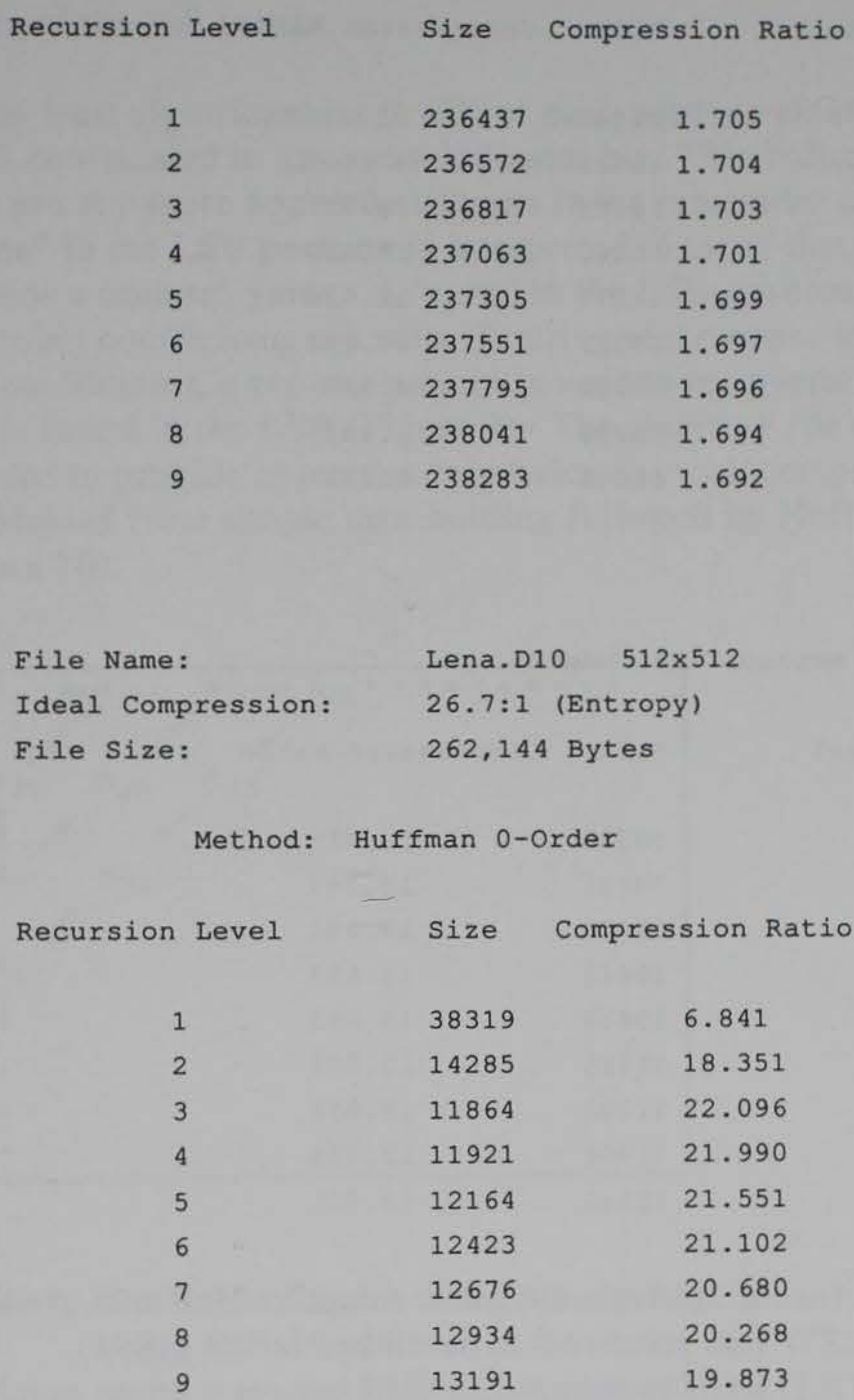

Method: Adaptive Huffman

Recursion Level

1
2
3
4
5
6
7
8
9

Size Compression Ratio

$\begin{array}{ll}37821 & 6.931 \\ 12993 & 20.176 \\ 11382 & 23.031 \\ 11546 & 22.704 \\ 11837 & 22.146 \\ 12135 & 21.602 \\ 12440 & 21.073 \\ 12745 & 20.568 \\ 13035 & 20.111\end{array}$




\begin{tabular}{|c|c|c|c|}
\hline \multirow[b]{2}{*}{ Recursion } & \multicolumn{2}{|c|}{ Method: LZW } & \multirow[b]{2}{*}{ Compression Ratio } \\
\hline & Level & Size & \\
\hline & 1 & 11492 & 22.811 \\
\hline & 2 & 15326 & 17.105 \\
\hline & 3 & 16601 & 15.791 \\
\hline & 4 & 19665 & 13.330 \\
\hline & 5 & 22337 & 11.736 \\
\hline & 6 & 25553 & 10.259 \\
\hline & 7 & 29169 & 8.987 \\
\hline & 8 & 33213 & 7.893 \\
\hline & 9 & 38045 & 6.890 \\
\hline & Method: & Arithme & =ic \\
\hline Recursion & Level & Size & Compression Ratio \\
\hline & 1 & 30055 & 13.416 \\
\hline & 2 & 30165 & 13.367 \\
\hline & 3 & 30404 & 13.262 \\
\hline & 4 & 30645 & 13.157 \\
\hline & 5 & 30886 & 13.055 \\
\hline & 6 & 31125 & 12.954 \\
\hline & 7 & 31363 & 12.856 \\
\hline & 8 & 31604 & 12.758 \\
\hline & 9 & 31846 & 12.661 \\
\hline
\end{tabular}

The source code for the Huffman 0-Order, Adaptive Huffman, Arithmetic coding, and LZW was presented in detail by Nelson (1991).

MOUSE.GS and GOLD.RGB represent standard images without much lossless compressibility, while LENA.D10 is a sparse file of DCT coefficients consisting mostly of zeroes. In general, if the entropy calculation is a large number (not much compressibility), then Huffman 0-Order can produce results close to the entropy and dynamic Huffman can exceed the entropy. For a sparse file, the Huffman 0-Order and Adaptive Huffman encoding methods are not very effective unless done at least twice recursively, while the LZW method gives better results on the first recursion. A recursion basically attempts to compress an already compressed file using the same method repeatedly. The fact that none of these methods adequately approaches the entropy for a sparse file indicates that there is room for improvement.

If it were possible to know the location of the zero entries in each $8 \times 8$ block, then only the nonzero elements would need to be stored, and the resulting file could be compressed even more tightly than indicated with a $p_{i} \cdot \log _{2} p_{\mathrm{i}}$ entropy calculation. The use of overhead bits to indicate the position of the nonzero elements in an $8 \times 8$ block of DCT coefficients can 
be minimized by taking advantage of the fact that the nonzero entries are clustered around the direct current (DC) component in the upper left-hand corner of the $8 \times 8$ matrix.

The least significant bit (LSB) of each nonzero element in the $8 \times 8$ matrix is confiscated to use as an indicator bit. This indicator bit tells if there are any more nonzero elements in the remainder of the $8 \times 8$ matrix. A "one" in the LSB position is interpreted to mean that the next element contains a nonzero value. A "zero" in the LSB position means that the remaining coefficients are zero. Starting at the upper left-hand corner (DC coefficient), a zig-zag pattern is used to transverse the matrix until a zero is found in the LSB (Figure 9). The resulting file can be Huffman encoded to provide approximately twice as much compression as would be obtained from simple thresholding followed by Huffman encoding (Figure 10).

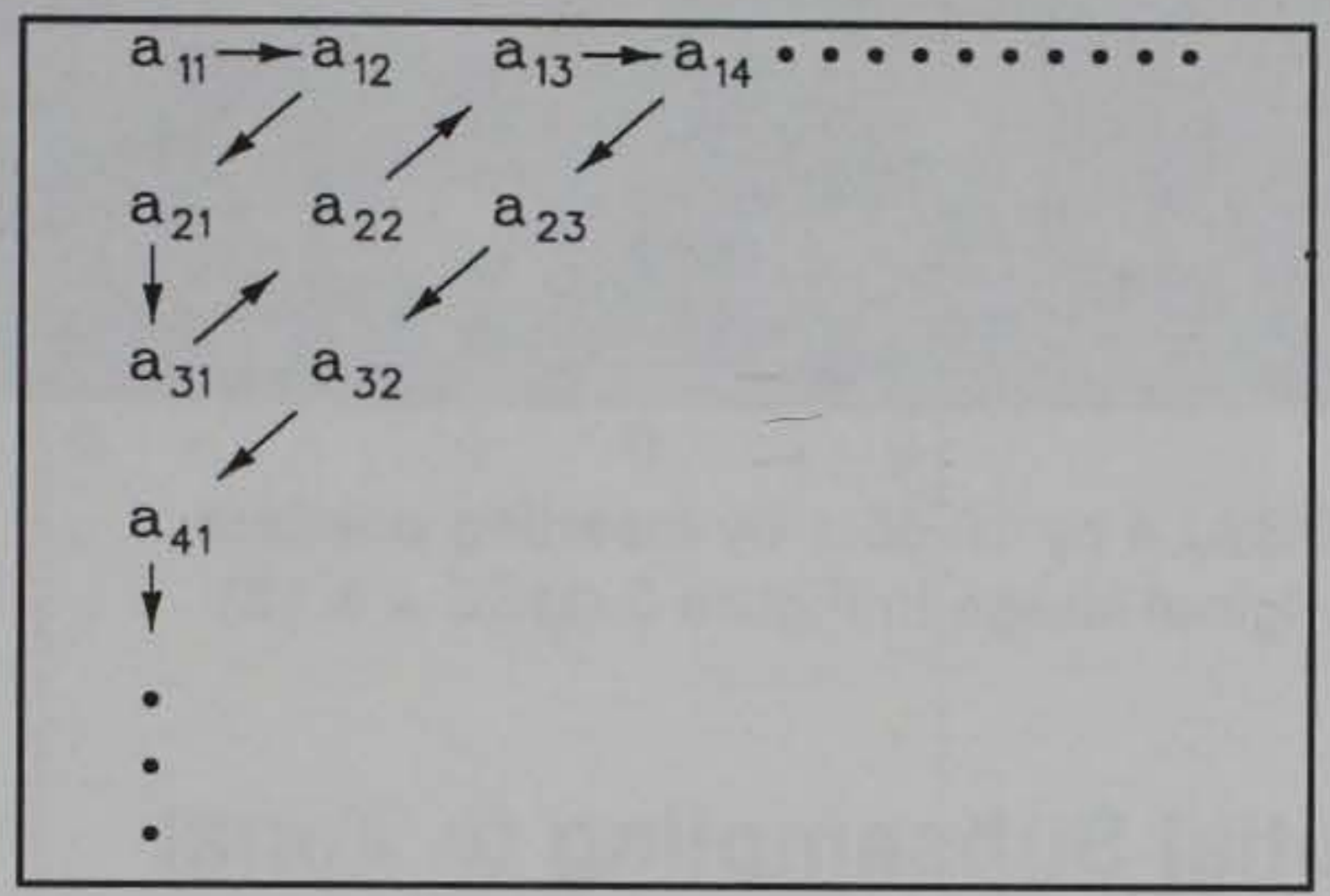

Figure 9. For each element along the zig-zag path, if the remainder of the matrix is zero, then the LSB of that element is zero, otherwise it is set to one

\section{Fourier Interpolation}

The Cosine Transform provides a method to increase the resolution of an image by interpolating between known pixels. The Fourier interpolation process (Figure 11) works as follows. To expand an image by $4: 1$, take the Cosine Transform of the original image in $8 \times 8$ blocks, and reconstruct the image using a 16×16 inverse Cosine Transform algorithm. The DCT coefficients in the original $8 \times 8$ transform block become the upper left quadrant in the $16 \times 16$ DCT block, with the other three quadrants padded with zeroes. The effect of taking the $16 \times 16$ inverse transform is equivalent to 2-D sinusoidal interpolation between the pixels in the original image. This process can be generalized to expand an image to any arbitrary size. 


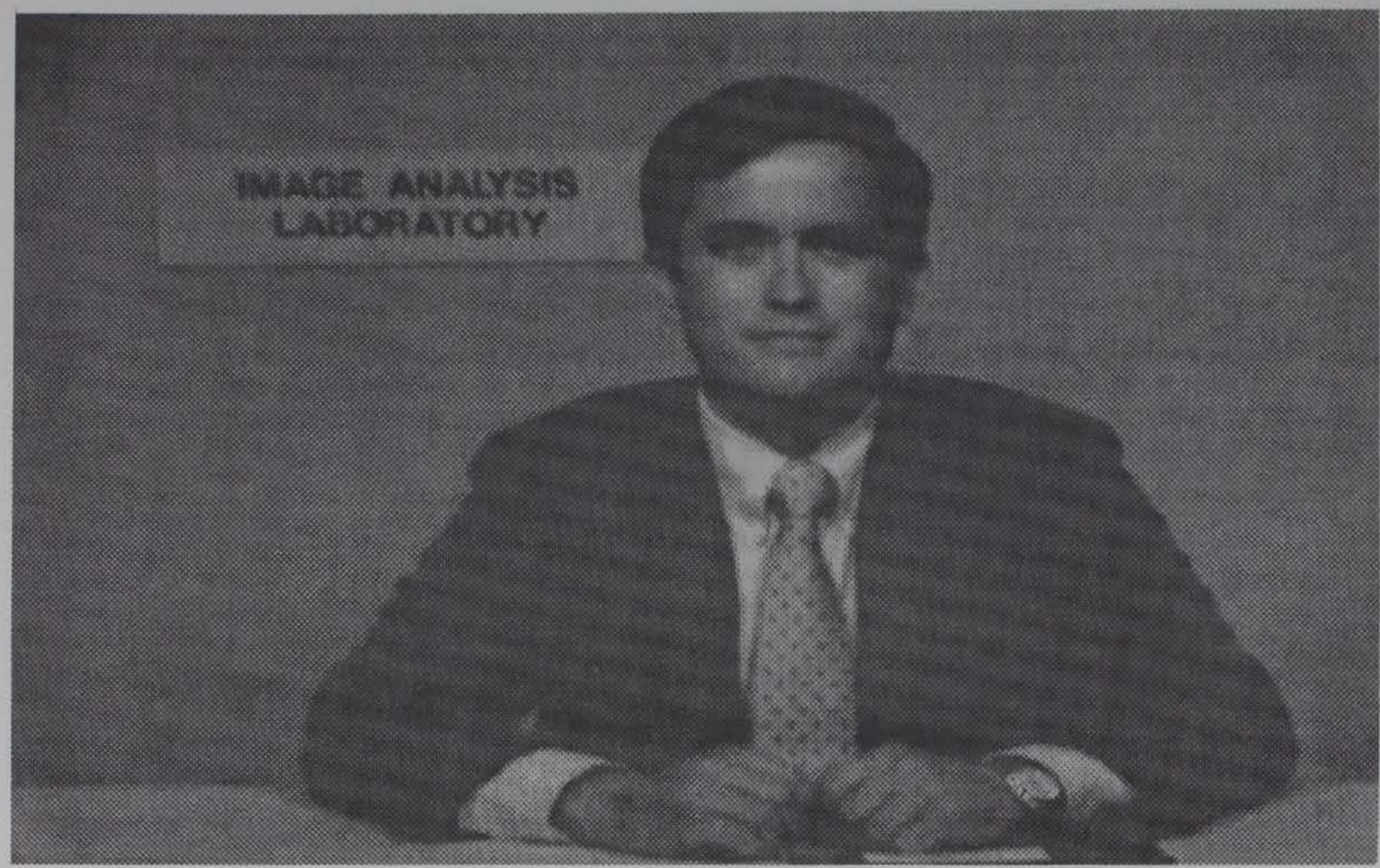

Figure 10. Compression and restoration of DBSJ.4 by $19.65: 1$ by inserting pointers along the zig-zag path with the original image in Figure 3 (MSE $=8.13$ )

\section{The Relation of Spatial Subsampling to Zonal Filtering}

The next logical step toward achieving high compression ratios would be to subsample an image at a rate of $16: 1$ and take the $8 \times 8$ DCT of the resulting subsampled image. If the DCT provided a compression of $6: 1$, then an overall compression of $96: 1$ is achieved. The restored image can be magnified by a $16: 1$ by using the Fourier interpolation technique previously discussed. Figure 12 shows one possible pattern to produce a 16:1 subsampled image prior to taking the DCT.

The scheme shown in Figure 12 does not provide an optimal way of taking the subsample. It is desired to create a 16:1 subsampled image in such a way that the interpolated image is optimally close to the original image. The zonal mask applied to the DCT transform creates an optimal subsampled image.

The process works as follows. Given an image, divide it into $32 \times 32$ blocks. Take the DCT of each $32 \times 32$ block, but retain only the first 16 elements $(4 \times 4)$ in the upper left-hand corner. The other 1,008 elements are forced to zero. 


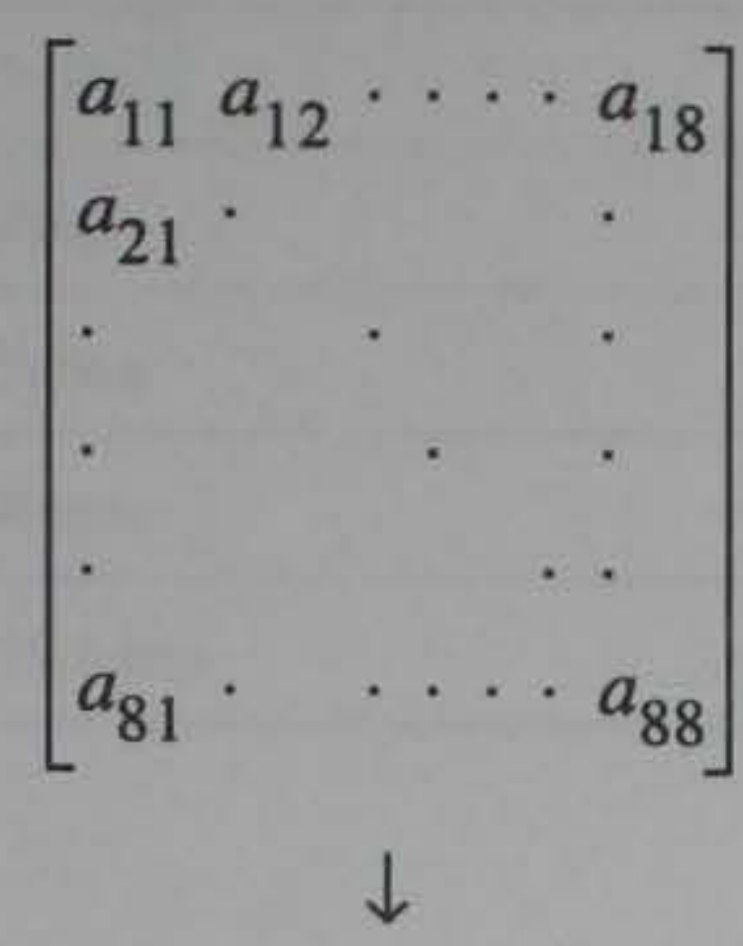

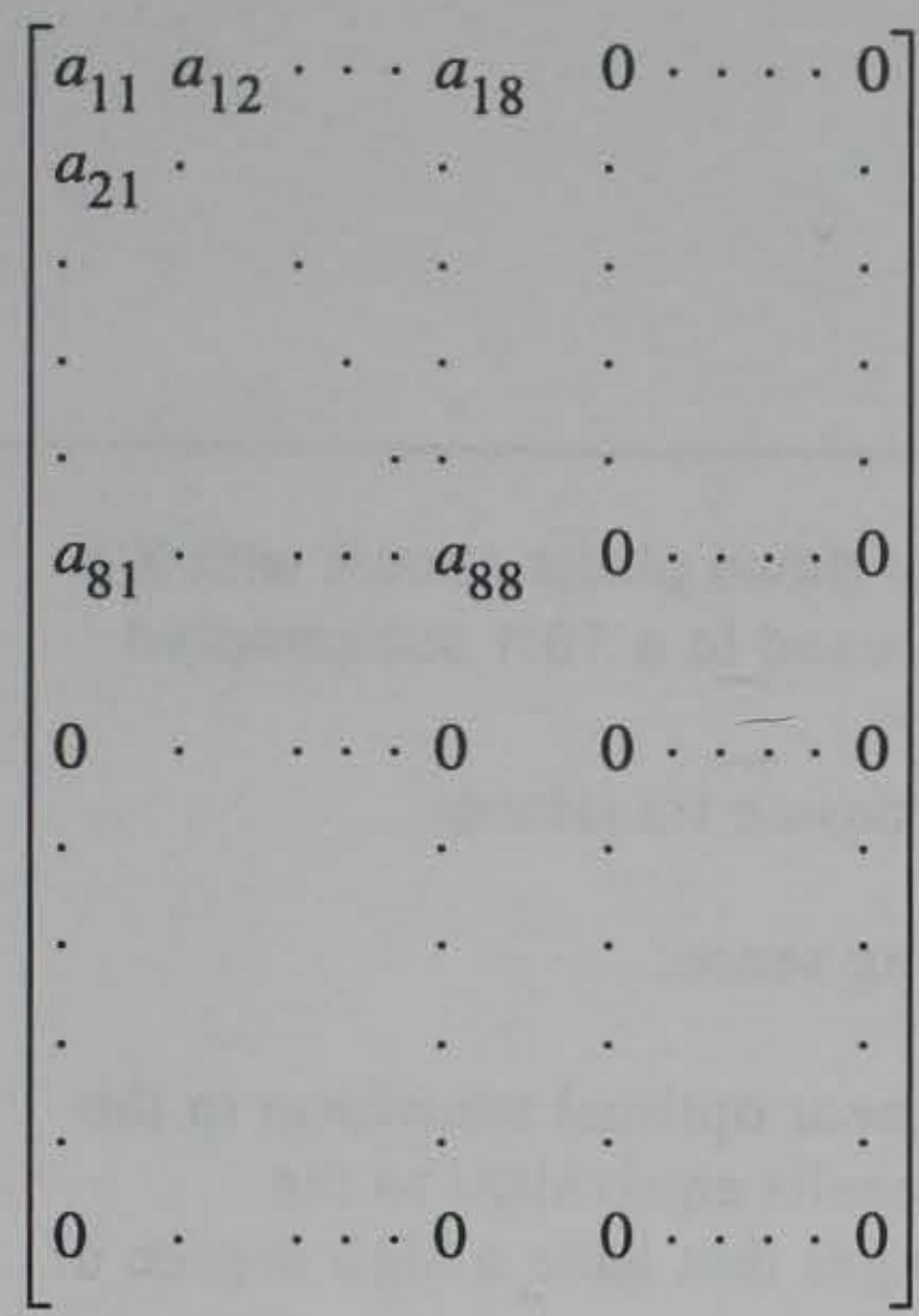

Figure 11. Fourier interpolation of an $8 \times 8$ transformed image by taking the inverse transform in $16 \times 16$ blocks with three quadrants padded with zeroes

If an inverse $4 \times 4$ DCT is performed on the remaining elements, then an optimal subsampled image is produced. In this case, the subsampling ratio is $64: 1$. Huffman encoding of the $4 \times 4$ DCT blocks will normally result in overall compression ratios of $80: 1$ to $160: 1$.

The restoration process includes:

a. Huffman decoding to recover the coefficients of each $4 \times 4$ transform block.

b. Creating $32 \times 32$ blocks from the $4 \times 4$ transform coefficients by padding the remaining 1,008 elements with zero. 


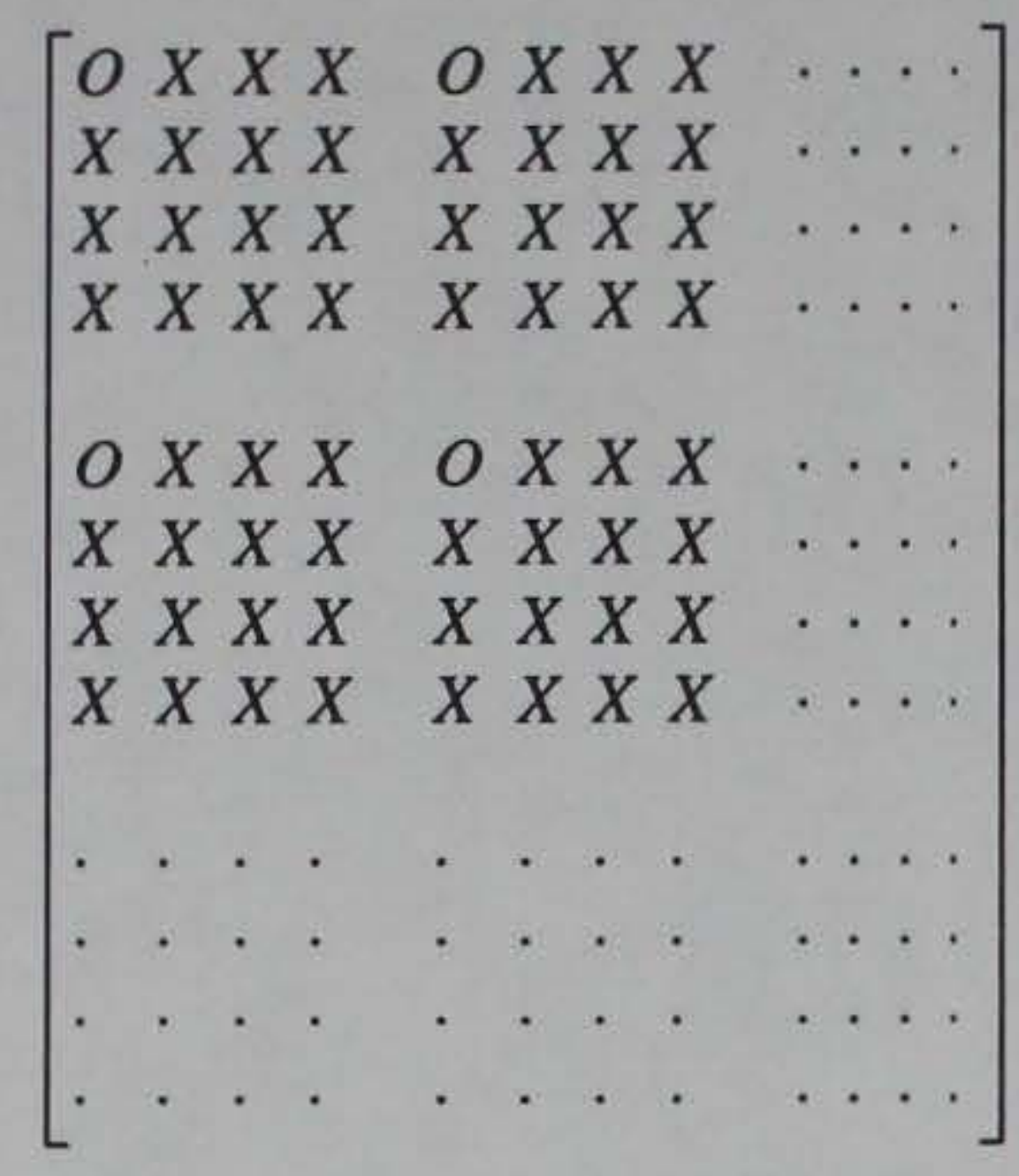

Figure 12. The original image consists of those pixels shown with X's and O's, but only the O's are used to a 16:1 subsampled image

c. Taking the inverse $32 \times 32$ discrete cosine transform.

This process is optimal in the following sense:

a. The discrete cosine transform is a near optimal transform in the mean-square sense and produces results equivalent to the Karhunen-Loeve transform for images that have a high degree of adjacent pixel correlation.

b. By utilizing the transform of the original image over $32 \times 32$ blocks, instead of $8 \times 8$ blocks, an advantage is taken of any correlation of pixels that may exist between adjacent $8 \times 8$ blocks. The JPEG algorithm cannot achieve compression rates as high because its block size is initially limited to $8 \times 8$.

c. A $4 \times 4$ zonal mask applied to the $32 \times 32$ transform creates an optimal subsample so that the interpolation process on reconstruction will be as close as possible to the original image.

A list of test images is shown below. Figures 13 through 17 display the original image beside the reconstructed image for comparison. In these figures, both the original and the reconstructed image have been scaled down to allow them to be placed side-by-side on a single page for comparison. In Figure 18, the left image is a zoom-in of the original PETRA; the right image is a zoom-in of the reconstructed image. This display is a true pixel-by-pixel comparison. 


\begin{tabular}{|l|l|l|}
\hline Image Name & Actual Size & Displayed Size \\
\hline Petra & $1,636 \times 2,152$ & $320 \times 340$ \\
\hline Vale & $2,523 \times 1,617$ & $504 \times 323$ \\
\hline Twins & $2,529 \times 1,578$ & $505 \times 315$ \\
\hline Room & $2,110 \times 2,695$ & $422 \times 539$ \\
\hline Turbans & $2,523 \times 1,617$ & $504 \times 323$ \\
\hline
\end{tabular}




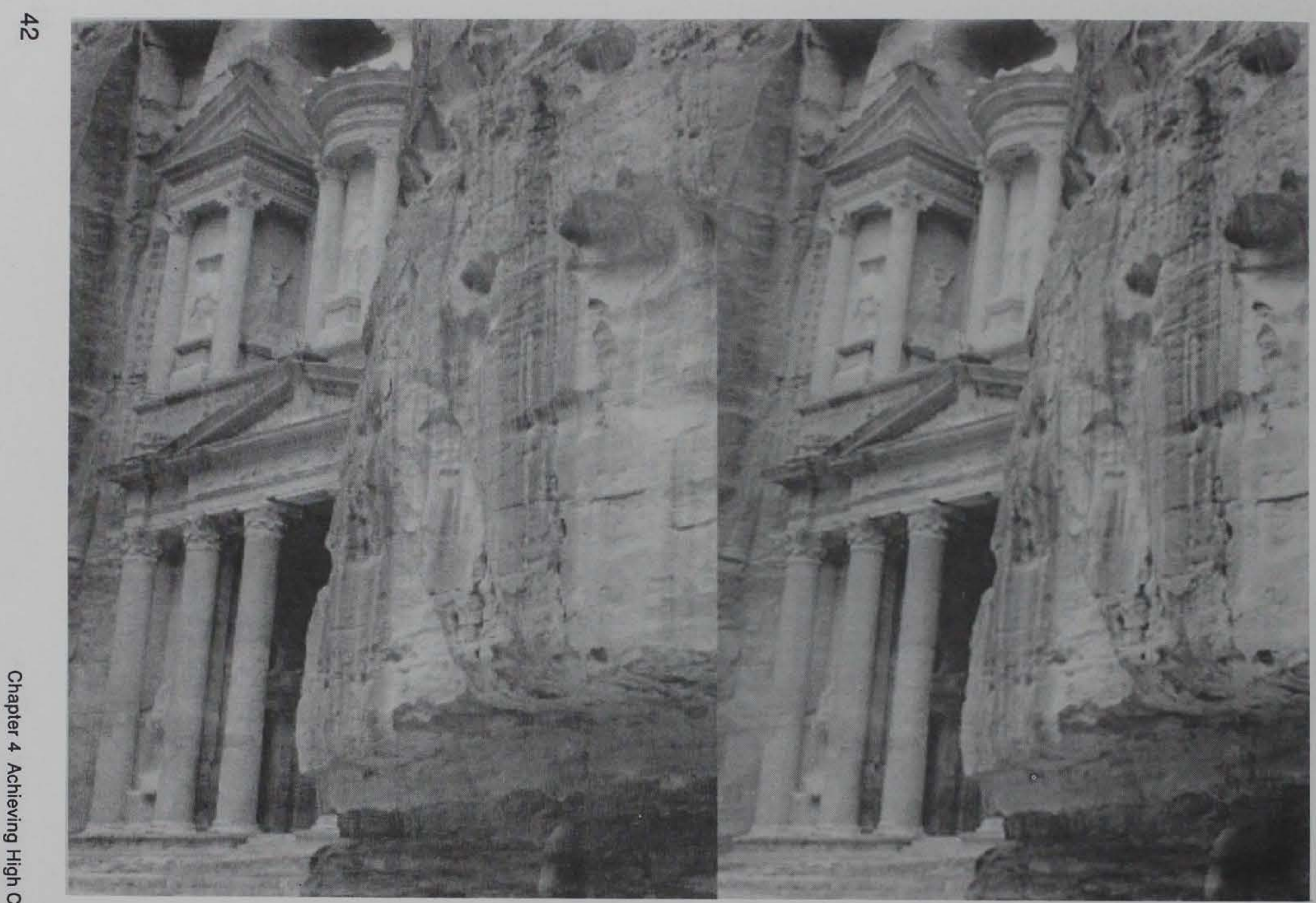

Figure 13. Petra - original image (left) and compressed 91.91:1 and restored (right) 

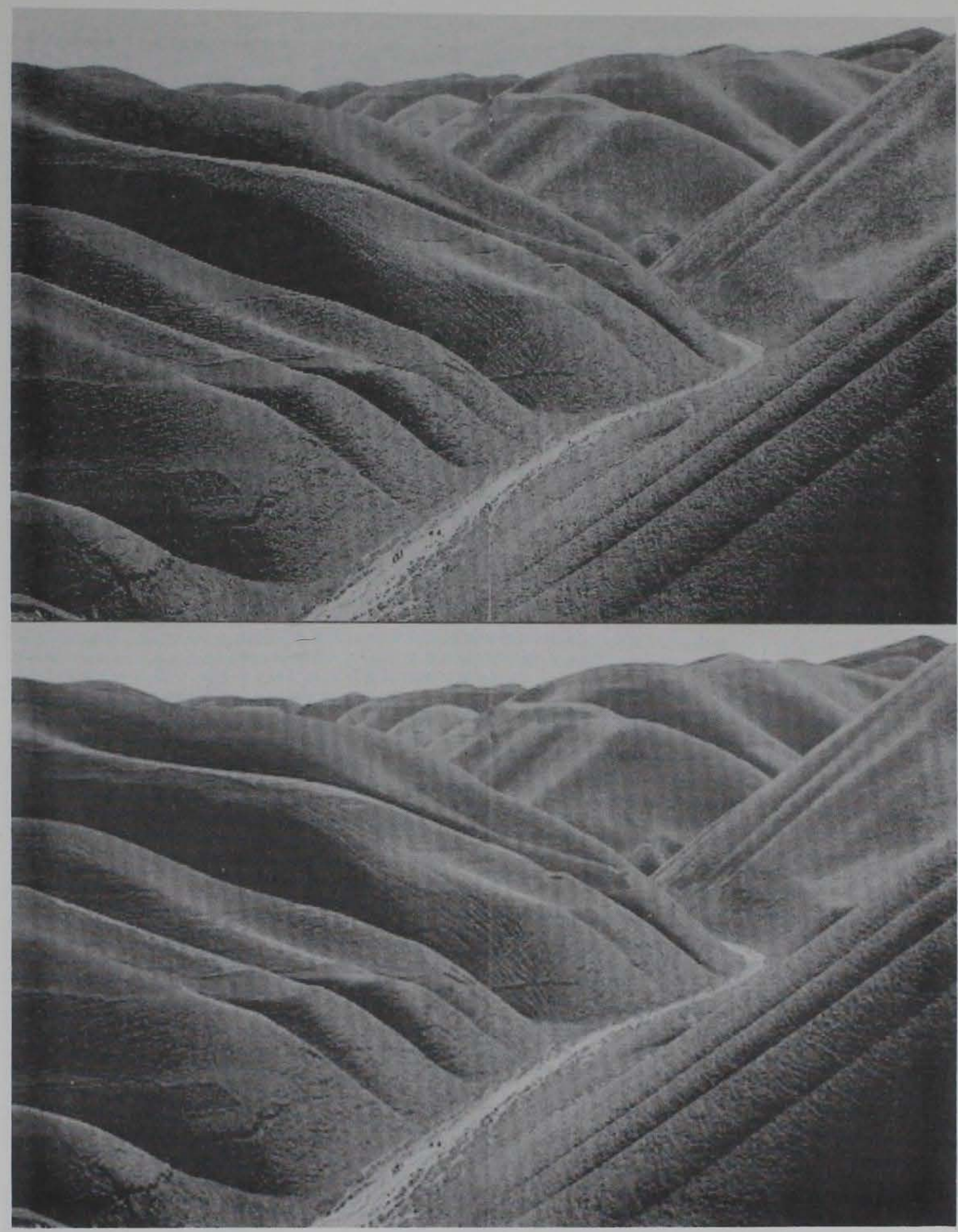

Figure 14. Vale - original image (top) and compressed 120.97:1 and restored (bottom) 


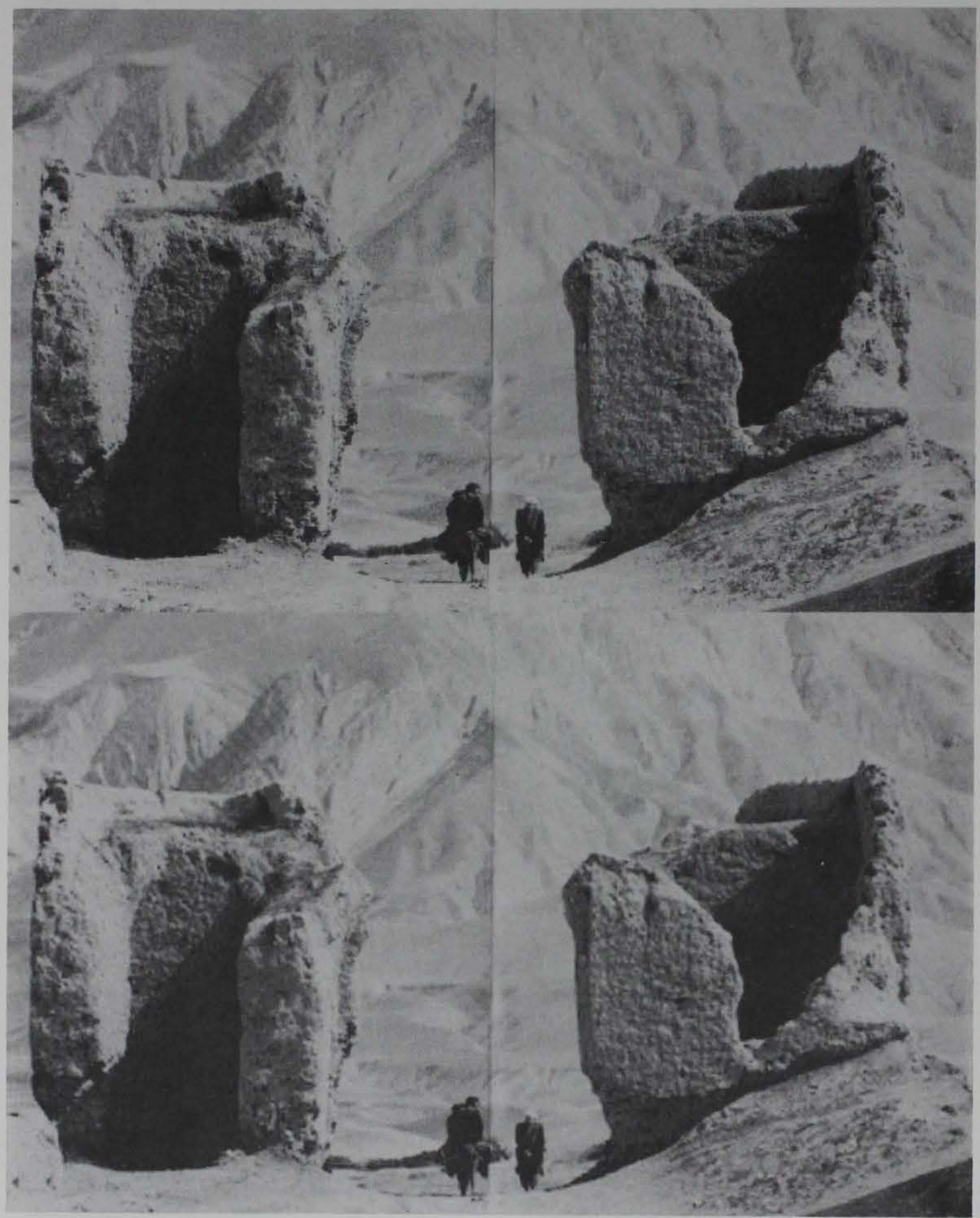

Figure 15. Twins - original image (top) and compressed $89.31: 1$ and restored (bottom) 


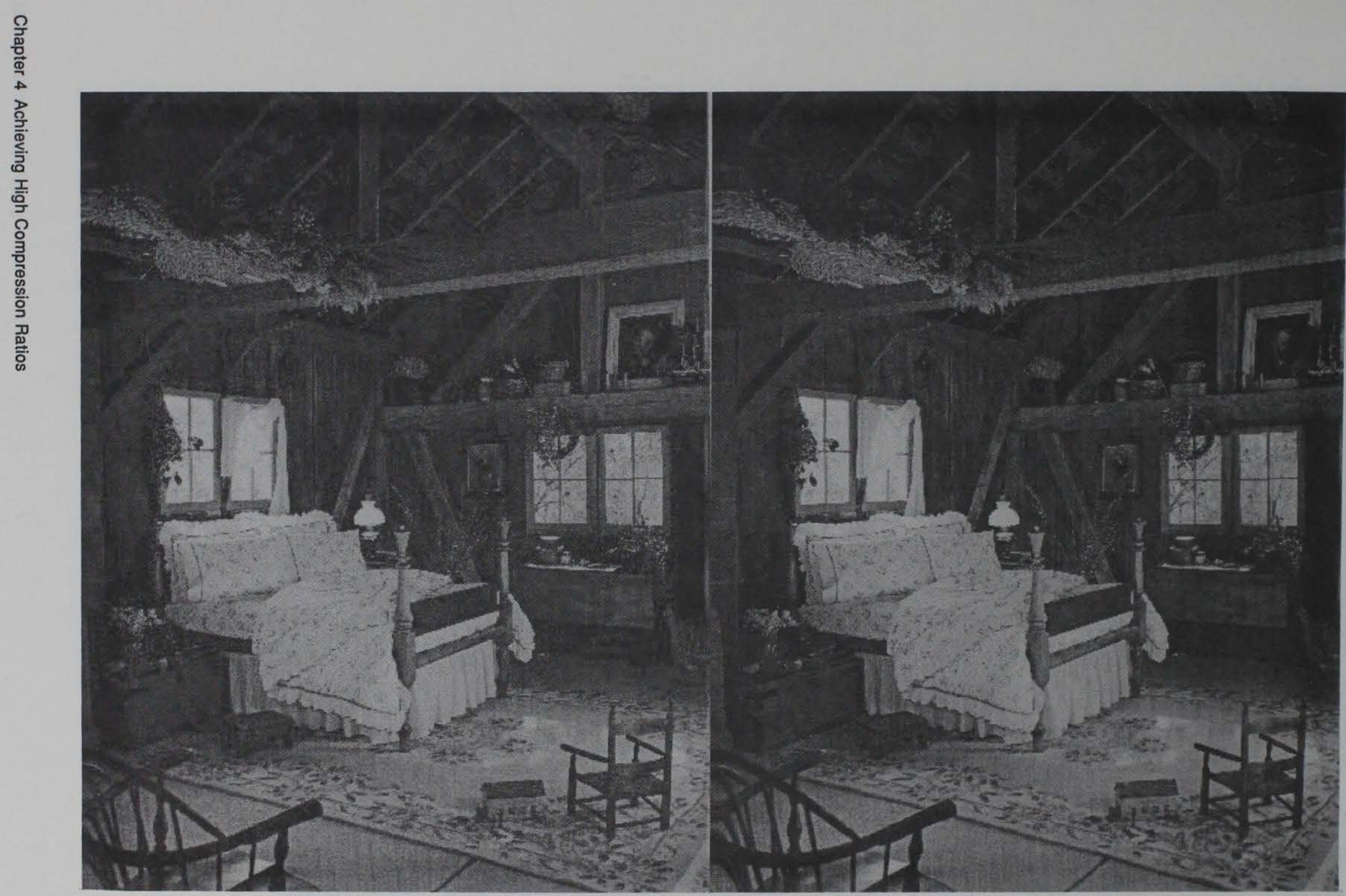

of Figure 16. Room - compressed 159:1 and restored image (left) and original (right) 


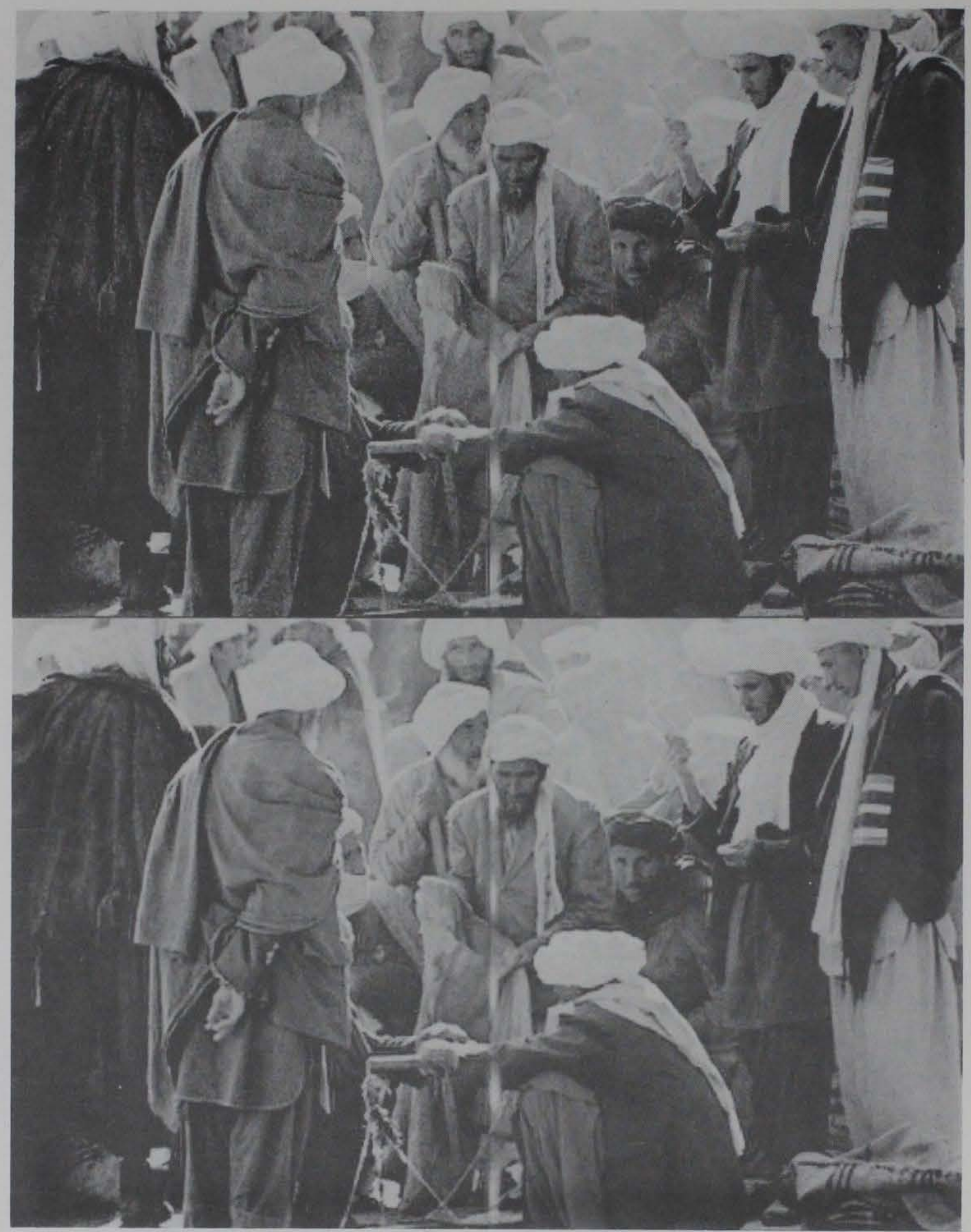

Figure 17. Turbans - original image (top) and compressed 100.9:1 and restored (bottom) 


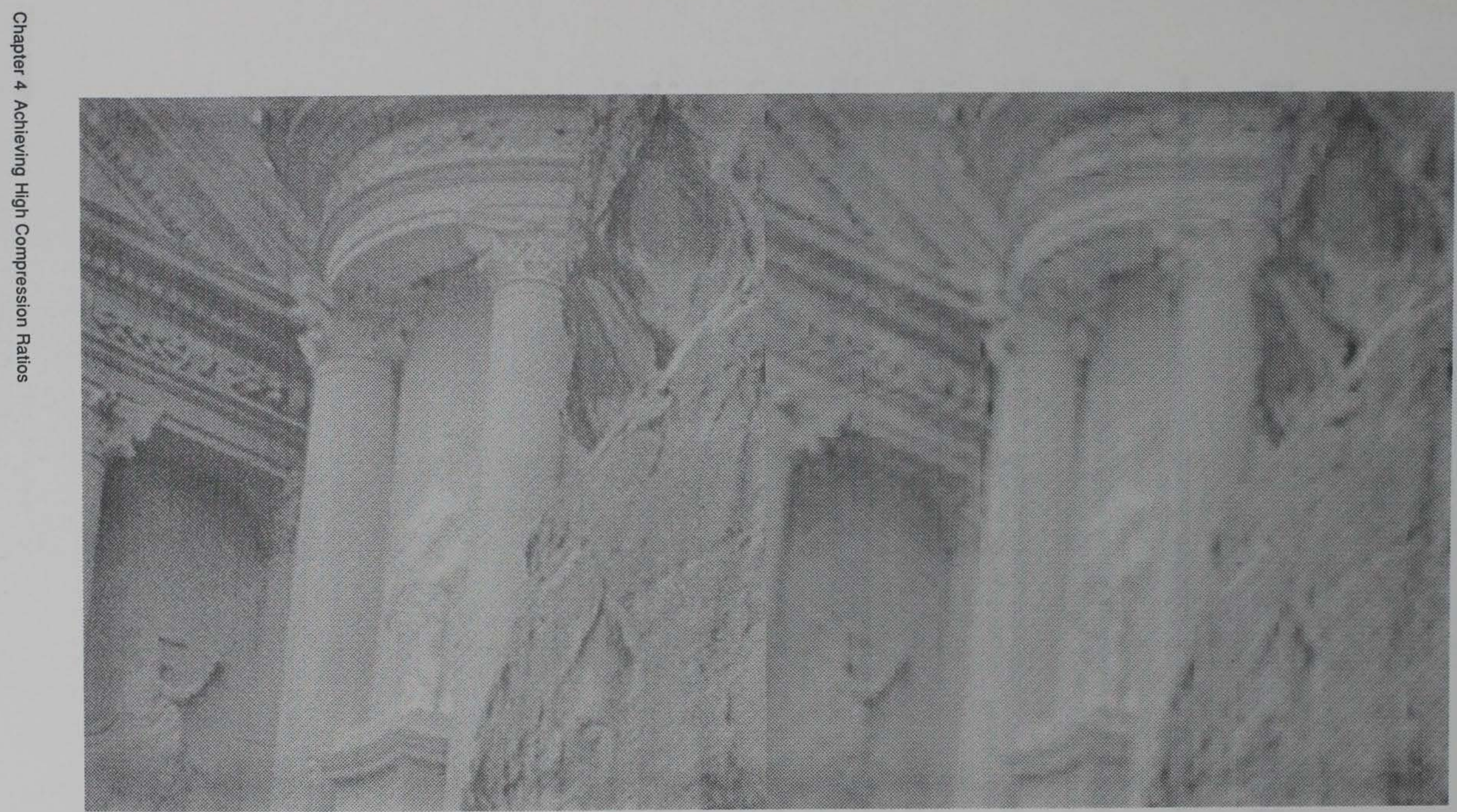

Figure 18. Zoom-in of the original PETRA (left); zoom-in of reconstucted image (right) 


\section{References}

Barnsley, M. (1988). Fractals everywhere, Academic Press, Inc., Norcross, GA.

Couch, L. (1983). Digital and analog communication systems, Macmillan Publishing Co., New York.

Jain, A. K. (1989). Fundamentals of digital image processing, PrenticeHall, Englewood Cliffs, NJ.

Nelson, M. (1991). The data compression book, M\&T Books, Redwood City, CA.

"Software listings," Dr. Dobb's Journal. (1991). M\&T Books, Redwood City, CA. 


\section{Bibliography}

Barnsley, M. (1992). "Methods and apparatus for image compression by iterated function system," U.S. Patent 4,941,193.

Ferraro, R. F. (1990). Programmer's guide to the EGA and VGA cards, 2nd ed., Addison-Wesley, Reading, MA.

Jacob, Lempel, A., and Ziv, J. (1977). "A universal algorithm for sequential data compression," IEEE Transactions on Information Theory.

. (1978). "Compression of individual sequences via variablerate coding," IEEE Transactions of Information Theory.

"NCSA image 3.1," National Center for Supercomputing Applications, University of Illinois, Urbana-Champaign.

“386-MATLAB user's guide.” (1990). The Mathworks, Inc. Natick, MA.

Welch, T. (1984). "A technique for high-performance data compression," IEEE Computer, 17(6), 8-19. 


\section{Appendix A \\ Compressions with the Cosine Transform and Singular Value Decomposition (SVD)}

The following set of images demonstrates increasing distortion because of increasing compression rates for two methods - the Cosine Transform and SVD. This display is by no means a comparison of the two methods. The Cosine Transform is performed in $8 \times 8$ blocks; whereas, SVD is performed over the entire image. If SVD were executed in $8 \times 8$ blocks, the method's ratio of compression to distortion would most likely improve.

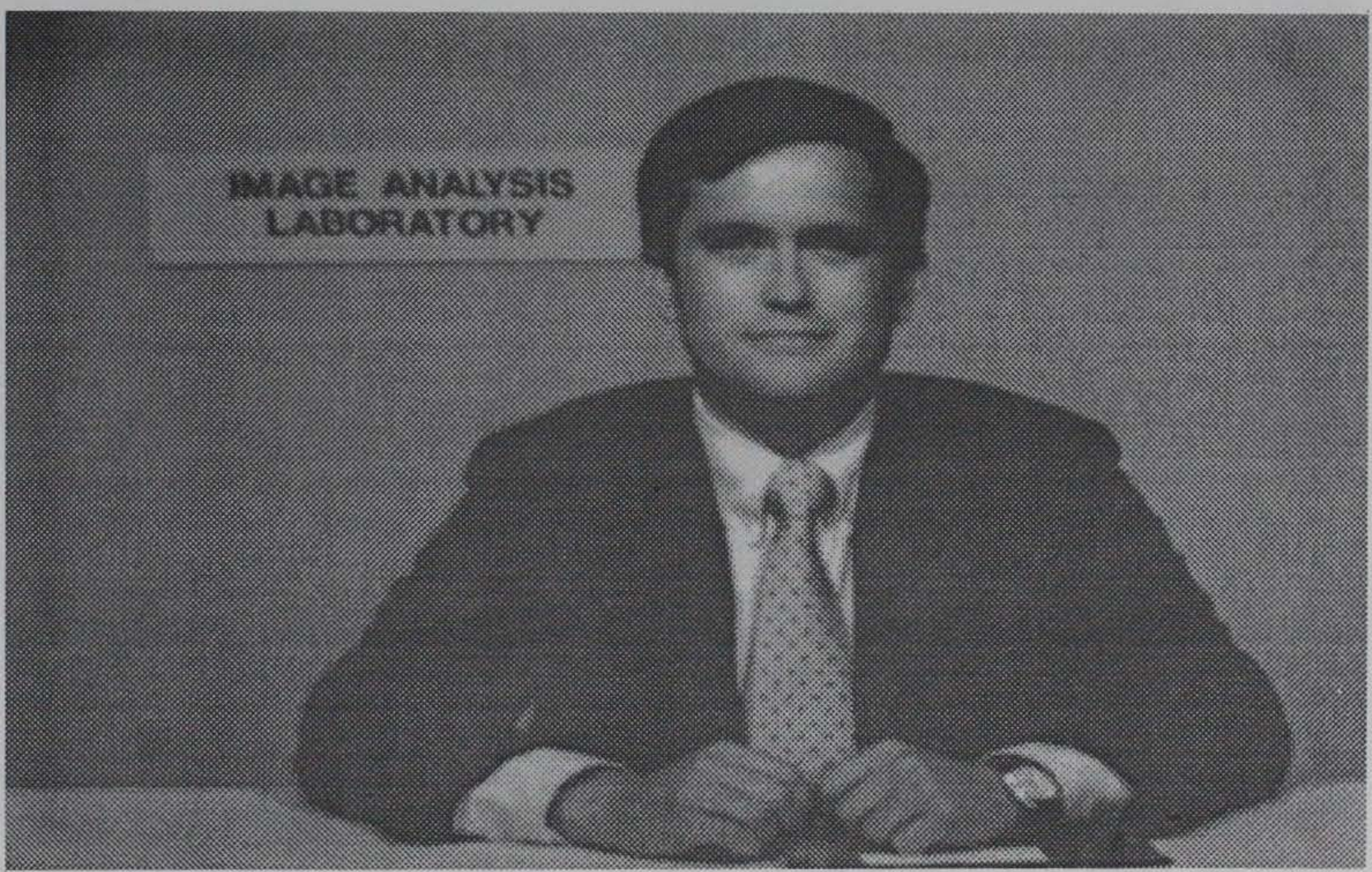

Figure A1. Original image 


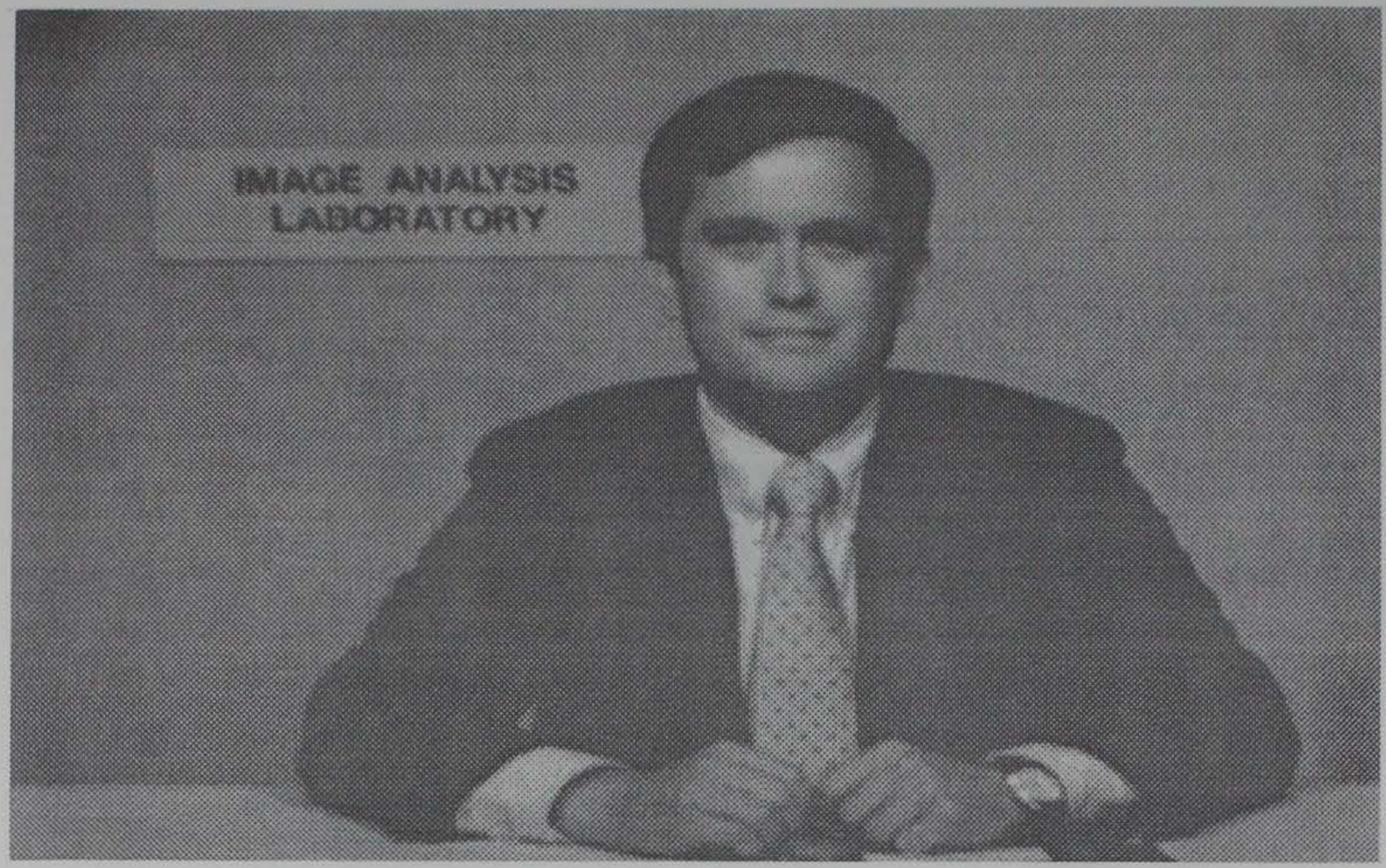

Figure A2. DCT encoding followed by Huffman encoding then Huffman decoding and inverse DCT (MSE $=2.734$; compression with Huffman encoding of DCT coefficients $=7.186: 1)$

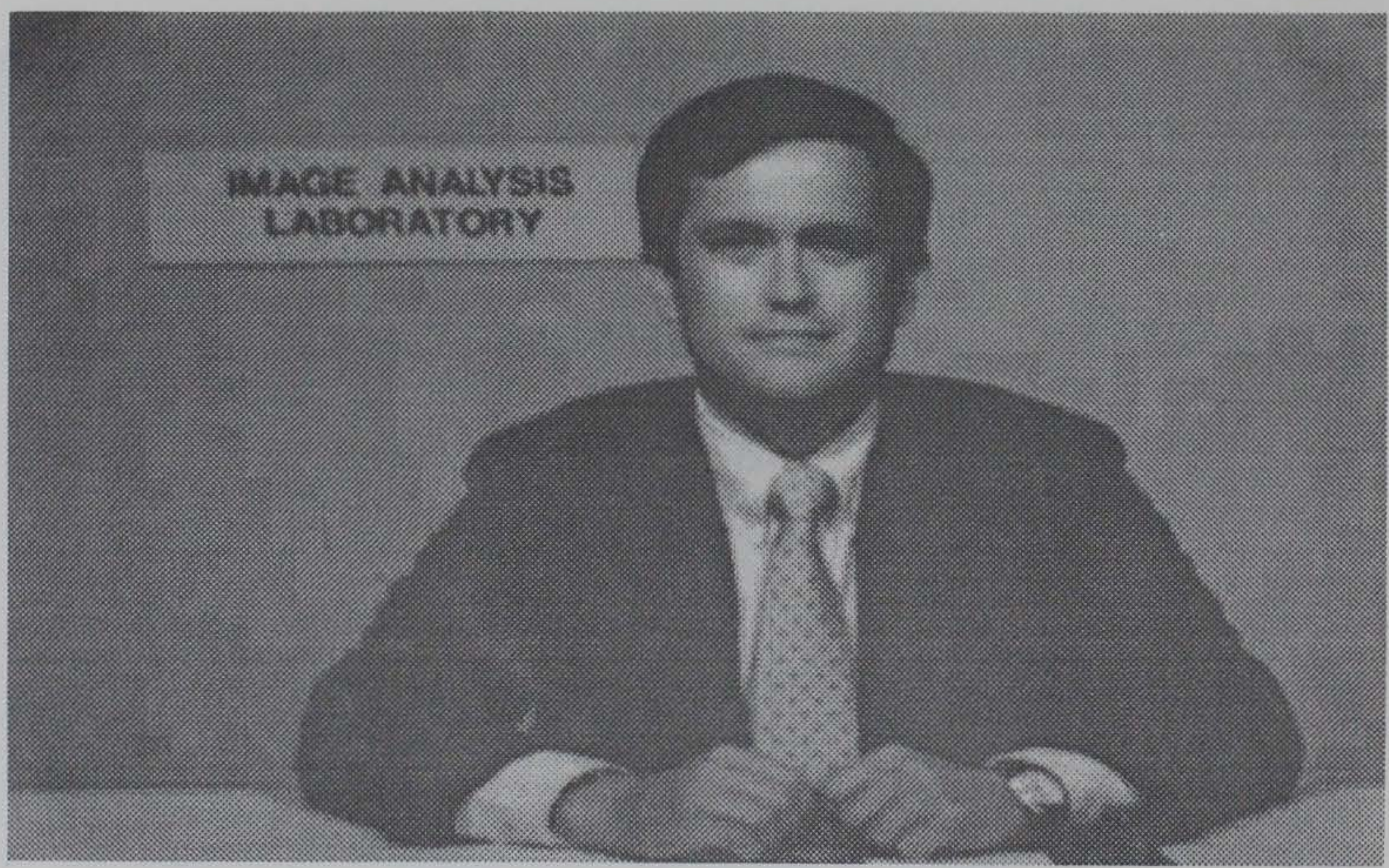

Figure A3. DCT encoding with a threshold of 1 followed by Huffman encoding, then Huffman decoding and inverse DCT (MSE $=5.859$; compression with Huffman encoding of DCT coefficients $=12.326: 1$ ) 


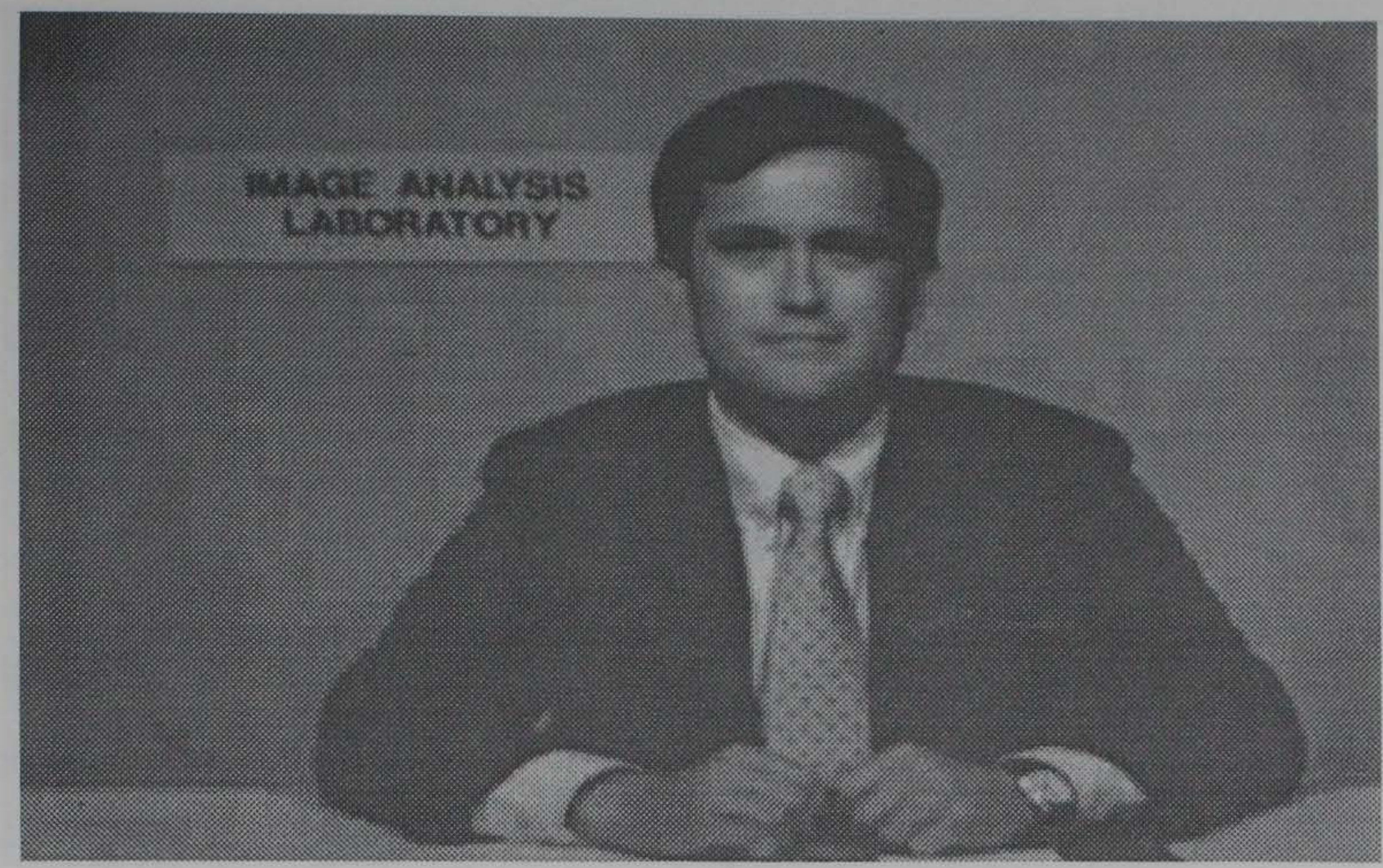

Figure A4. DCT encoding with a threshold of 2 followed by Huffman encoding, then Huffman decoding and inverse DCT (MSE $=9.818$; compression with Huffman encoding of DCT coefficients $=15.659: 1$ )

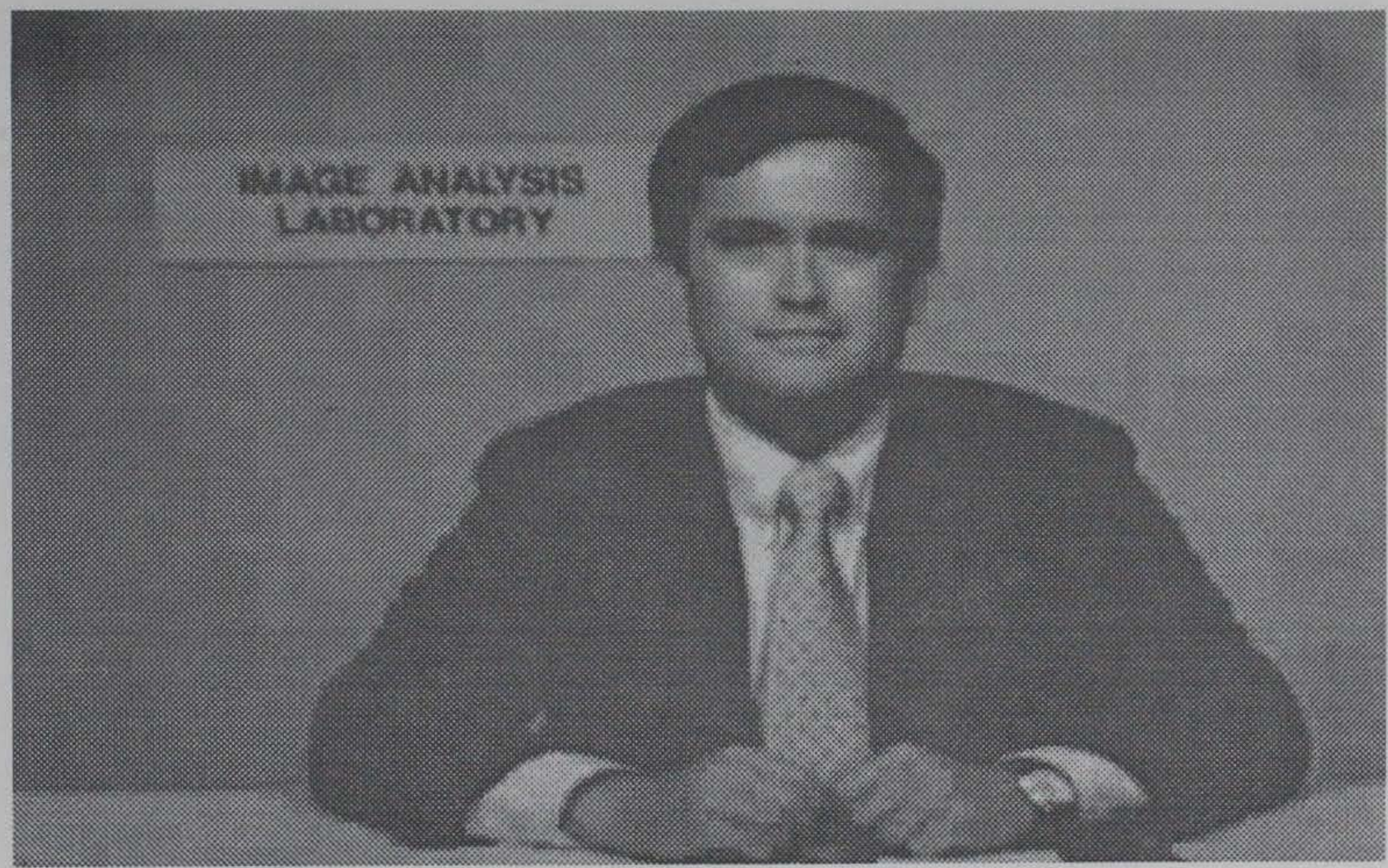

Figure A5. DCT encoding with a threshold of 3 followed by Huffman encoding, then Huffman decoding and inverse DCT (MSE = 14.544; compression with Huffman encoding of DCT coefficients $=18.488: 1$ ) 


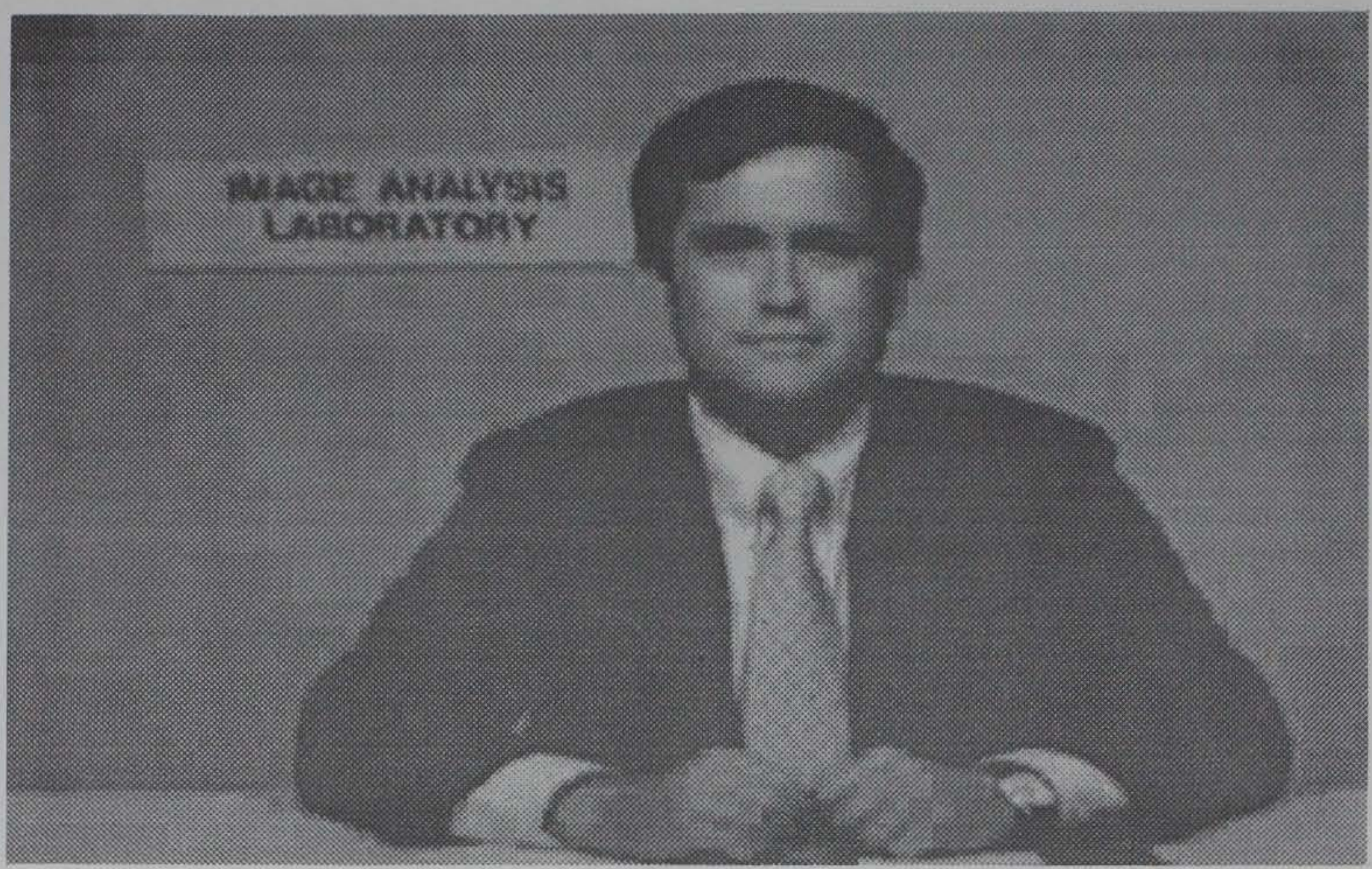

Figure A6. DCT encoding with a threshold of 4 followed by Huffman encoding, then Huffman decoding and inverse DCT (MSE $=20.047$; compression with Huffman encoding of DCT coefficients $=21.13: 1$ )

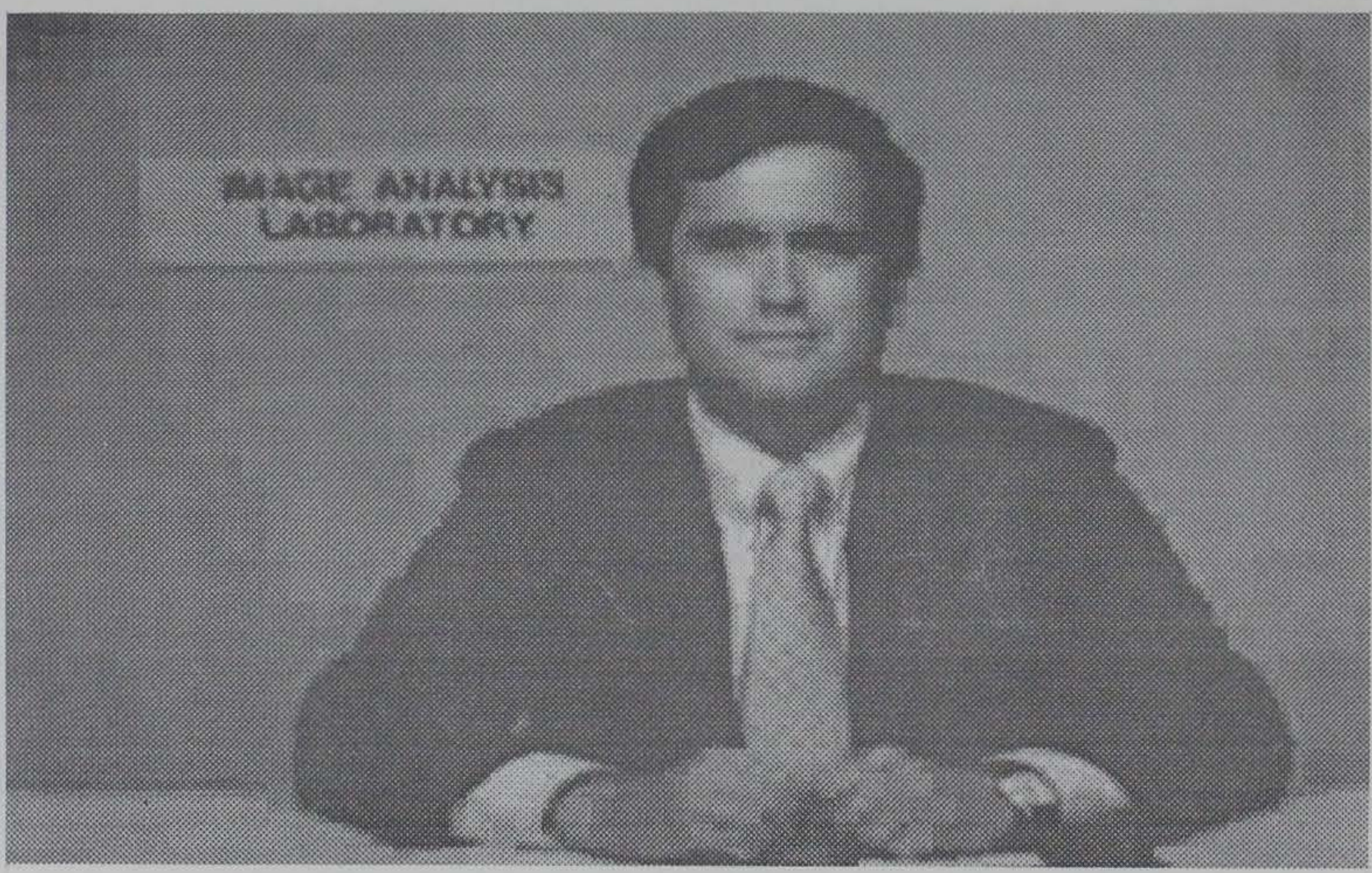

Figure A7. DCT encoding with a threshold of 5 followed by Huffman encoding, then Huffman decoding and inverse DCT (MSE $=25.39$; compression with Huffman encoding of DCT coefficients $=23.34: 1$ ) 


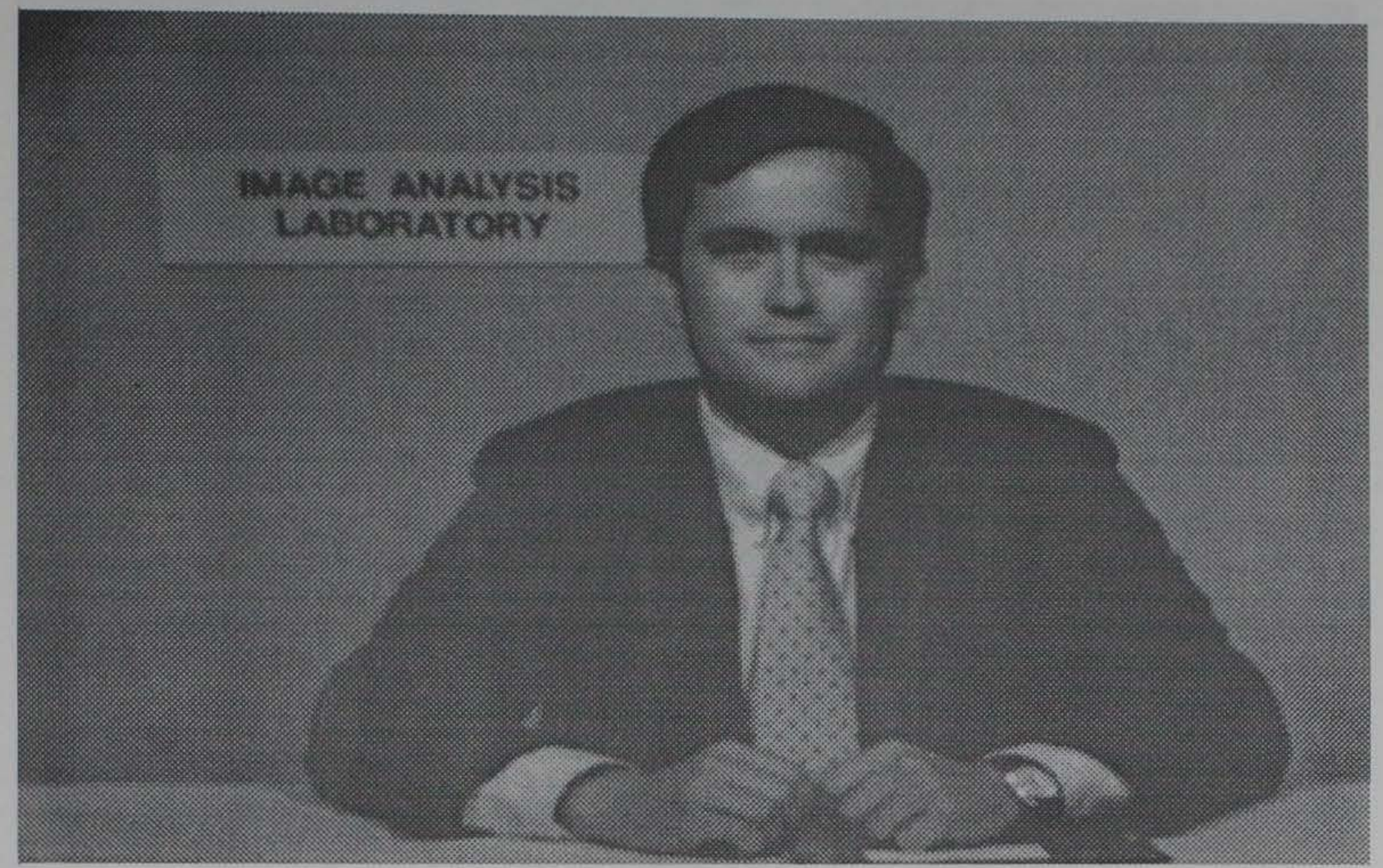

Figure A8. A 2:1 compression with SVD (MSE $=0.765$ )

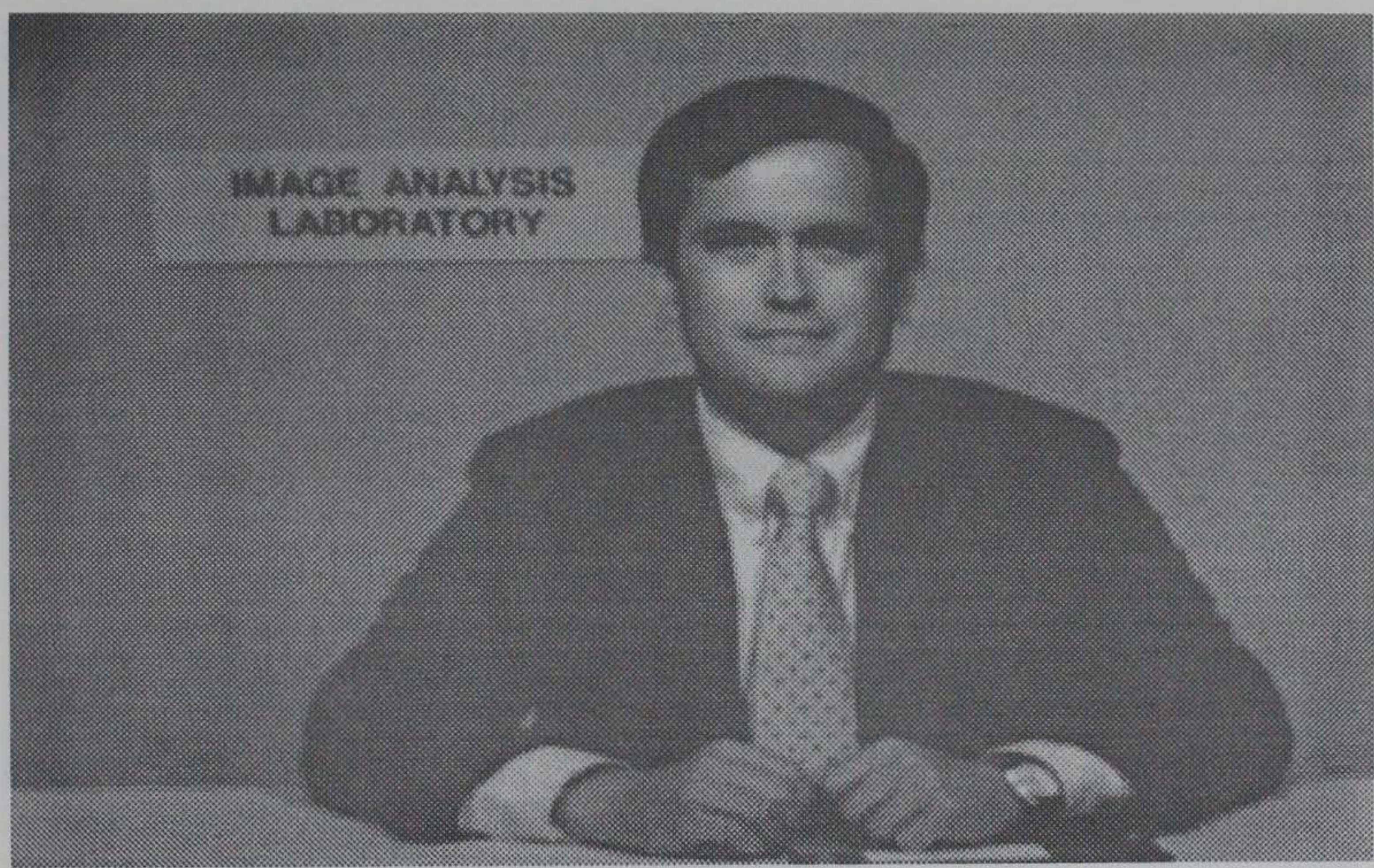

Figure A9. A 4:1 compression with SVD (MSE $=3.802$ ) 


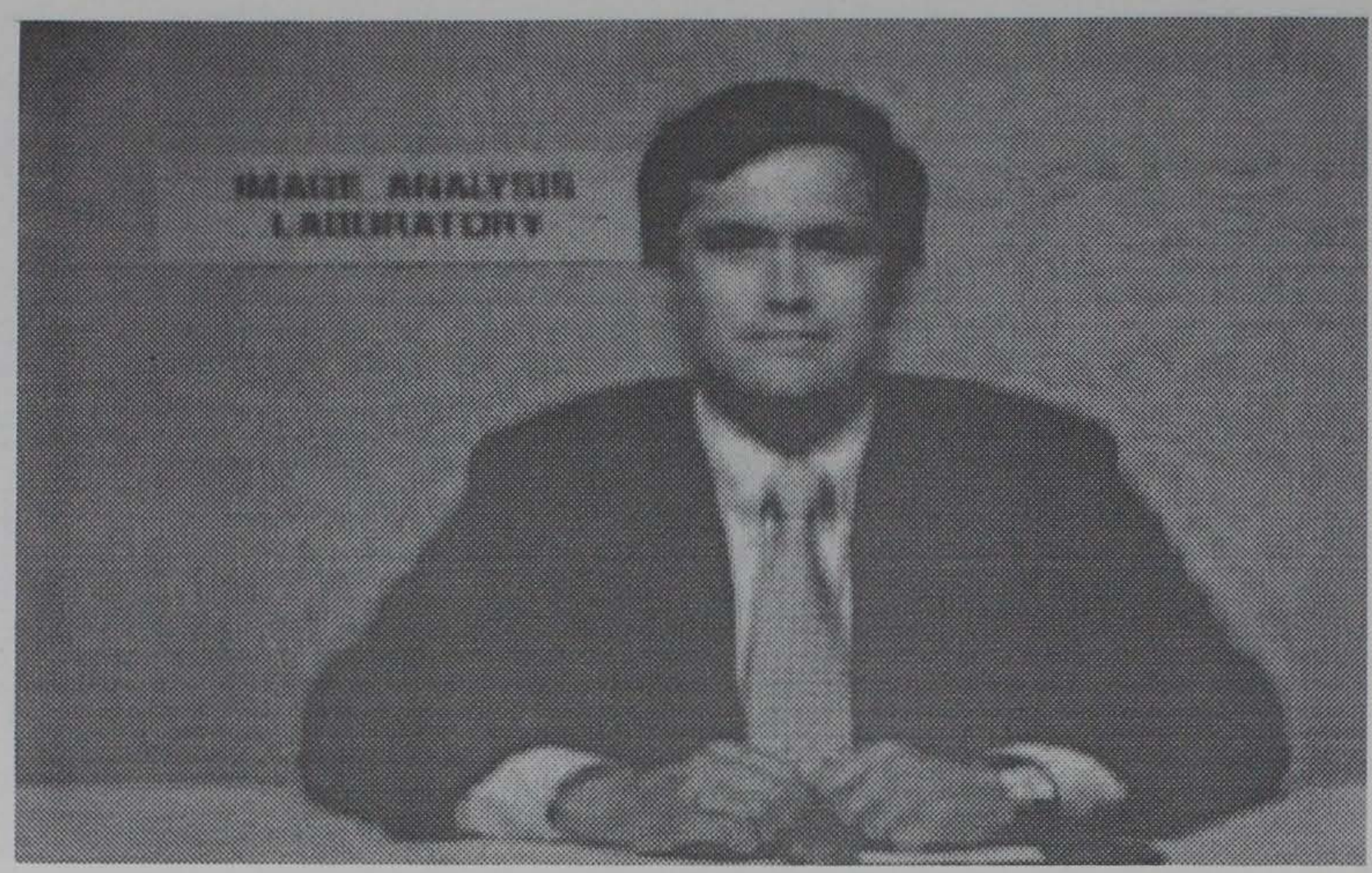

Figure A10. A 10:1 compression with SVD (MSE $=29.584$ )

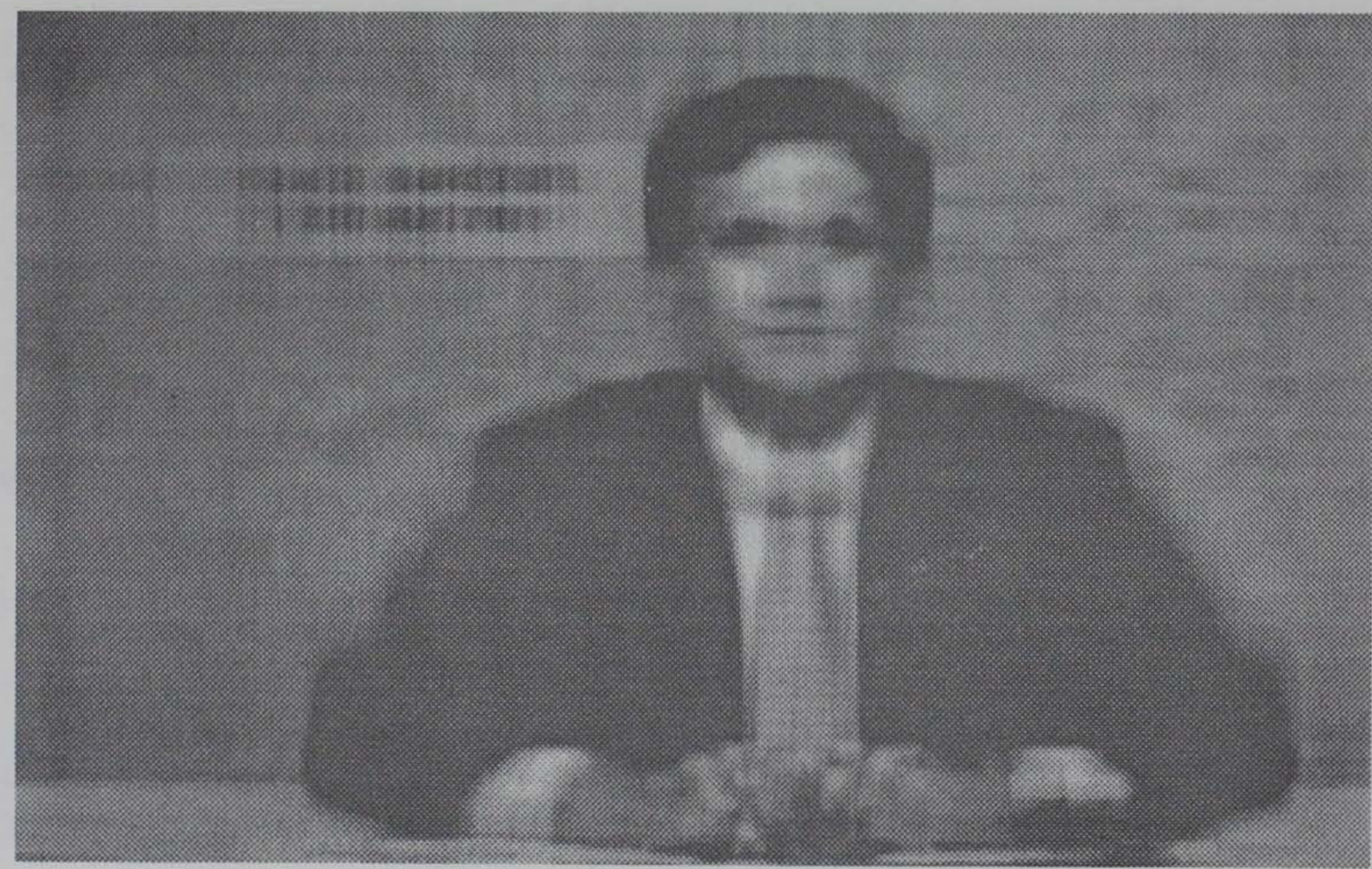

Figure A11. A 25:1 compression with SVD (MSE = 124.2) 


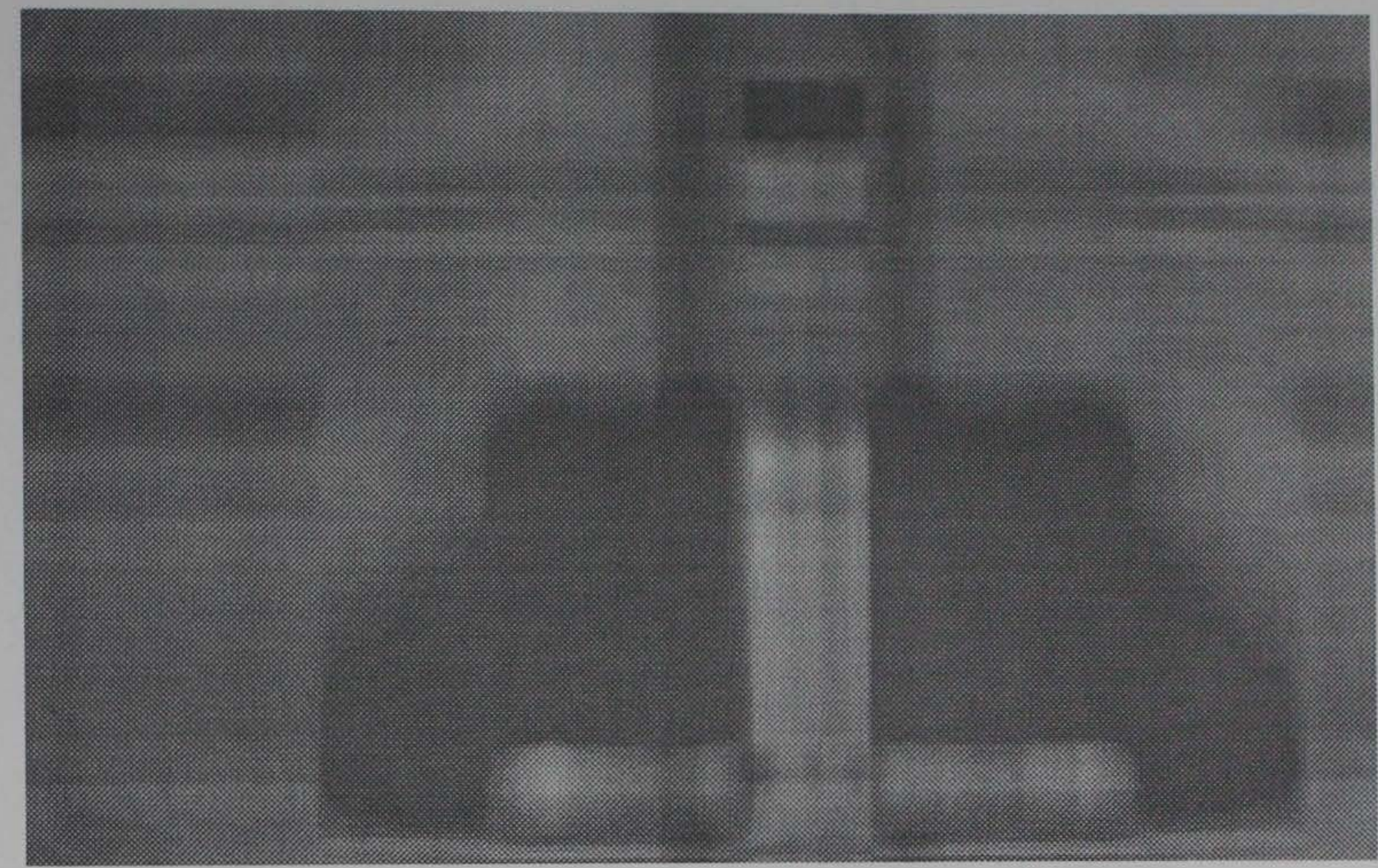

Figure A12. A 100:1 compression with SVD (MSE $=552.95)$ 


\section{Appendix B "Image Lab" Software User's Guide}

File

Copy File, Delete File, and Rename File perform similarly to their Disk Operating System (DOS) equivalents. Copy File copies file information from one file to another. Delete File deletes a file. Rename File moves file information from one file to another.

Load File requests a image file name in either the RGB format or the greyscale format. Image width and height are requested. This selection is a prerequisite to displaying an image. Once an image file is loaded, it does not need to be reloaded; the most recent image file and its dimensions are stored in global memory. For example, if a different display mode is desired, change the mode in "Options," and then select Display Full Image under "View"; the image will then be redisplayed without requiring the user to reload the image information.

Load Palette requests an RGB or greyscale palette name. An RGB palette must list the 256 intensities for (a) red, (b) green, and (c) blue for a total of 768 entries. A RGB pixel is translated by the following mask: $R$ R R G G G B B. Bits 5-7 indicate the intensity of red, bit 7 being the MSB. Bits 2-4 indicate the intensity of green, bit 4 being the MSB. Bits $0-1$ indicate the intensity of blue, bit 1 being the MSB. A greyscale palette must list the 256 grey intensities and repeat the list twice for a total of 768 entries. (Note: To obtain any shade of grey, the intensities of red, green, and blue must be equal.) All palettes must be 768 bytes in size. No stray bits are allowed! Once a palette is loaded, it remains in global memory; therefore, the palette does not require reloading from one image to the next. The default palette is GRAYTEST.PAL.

Quit ends the use of "Image Lab." 


\section{View}

Show Red shows only the red intensities. Show Green shows only the green intensities. Show Blue shows only the blue intensities. Show Bit Plane 1-7 masks out all bits except the requested one. If the bit is present in a pixel, white will be displayed, else black will be displayed. Display Full Image redisplays the original image.

\section{Analysis}

Calculate Entropy calculates the entropy and predicts the theoretical compression ratio for an image or processed file. The theoretical compression ratio is based on Huffman-0. Chop Image File allows a subimage to be created from an image file. The original file name and dimensions are requested along with the output file name, starting and ending horizontal coordinates, and the starting and ending vertical coordinates. The top left pixel in the original file is located at $X=1, Y=1$. The starting and ending bounds are inclusive. Compare Files compares the pixels of two identically sized files. The threshold is the lowest difference that is to be flagged. The output will display four aspects of the differing pixels. Pixel 1 is the value of the pixel in the first file. Pixel 2 is the value of the pixel in the second file. $\mathrm{X}$ is the horizontal coordinate. $\mathrm{Y}$ is the vertical coordinate. The top left pixel is located at $X=0, Y=0$. This selection is helpful in analyzing images processed by lossy techniques. The original and reconstructed images can be compared on a pixel-by-pixel basis.

Compression Ratio tells the original file size, the compressed file size, and the ratio. Difference Images creates a difference image between original and reconstructed images. Zero error is represented by gray or intensity 128 . Increasing positive error is represented by increasing intensity, and increasing negative error is represented by decreasing intensity. Histogram makes a text histogram of the pixels in an file. Mean Squared Error finds the MSE between two image files. The two files do not have to be the same size. For color processed images, this selection gives distorted results since the color procedures work with RED bits, GREEN bits, and BLUE bits instead of full 8-bit pixels. Paste Image Files: Horizontally pastes two images side by side; Paste Image Files: Vertically pastes one image above the other. The two images do not have to be the same height or width. This selection is helpful in visually comparing original and reconstructed images.

\section{Lossless}

Four options are available for compression and decompression: (a) Huffman-0, (b) Adaptive Huffman, (c) LZW, and (d) Arithmetic. If a 
file is compressed in one option, it must be decompressed in the same option.

\section{Lossy}

Three transforms and inverse transforms are available for compression and decompression: (a) Cosine, (b) Hadamard, and (c) Sine. If a file is compressed in one option, it must be decompressed in the same option. Black \& White processes the image as a greyscale image. For compression, the reconstructible DCT will be located in *.1*; the displayable DCT will be located in the output file specified. For decompression, an *.1* file must exist to reconstruct, or an *.d* file must exist assuming that the image can be cut evenly into blocks specified by the lossy block mode being used. Color processes the image as an RGB image by translating to the $Y, C_{b}, C_{r}$ format as shown in Equations B1-B3.

$$
\begin{aligned}
& Y=0.299 * R+0.587 * G+0.114 * B \\
& C_{b}=-0.16874 * R-0.33126 * G+0.5 * B \\
& C_{r}=0.5 * R-0.41869 * G-0.08131 * B
\end{aligned}
$$

RGB format is regained with Equations B4-B6.

$$
\begin{aligned}
& R=Y+1.402 * C_{r} \\
& G=Y-0.34414 * C_{b}-0.71414 * C_{r} \\
& B=Y+1.772 * C_{b}
\end{aligned}
$$

For compression, the displayable DCT's will be located in *.yyy, *.ccb, and *.ccr; the reconstructible DCT's will be located in *.1yy, *.1cb, and $* .1 \mathrm{cr}$. For decompression, *.1yy, *.1cb, and *.1cr files must exist to reconstruct. File names are not a problem as long as all processing is done by this software!

In compression, the DCT is manipulated by two factors: thresholding and zonal filtering. If the absolute value of a DCT entry is less than or equal to the threshold, that entry is set to zero. Zonal filtering is more complicated. The zonal filter level tells what coefficients to keep in each DCT square. For example, if the lossy mode is set to $8 \times 8$ and the zonal filter level equals 4 , each $8 \times 8$ block of the DCT will retain the 16 entries in the top left corner that make a $4 \times 4$ square. All coefficients outside the $4 \times 4$ are set to zero. To disable zonal filtering, select level 8 for $8 \times 8$ mode, 16 for $16 \times 16$ mode, and 32 for $32 \times 32$ mode. 


\section{Options}

Set Video Mode displays the possible video modes and allows one to be selected. The default mode is set to the highest resolution that the software can find. If the PC's graphics card is not a Super VGA card, most likely, the only mode that will work is $320 \times 200$. If images will not display, set the video mode to $320 \times 200$.

Set Lossy Mode allows three different lossy modes to be selected: $8 \times 8,16 \times 16$, and $32 \times 32$. Each mode represents the block size that the image will be cut into to be processed. The image file does not have to be cut evenly into squares of the selected block size. For example, a 17x17 image can be processed in any of the lossy modes. The $8 \times 8$ and $16 \times 16$ modes can process image files with a maximum width of 1,024 pixels. The $32 \times 32$ mode is restricted to a maximum width of 640 pixels. The height is boundless. The $8 \times 8$ processes files in one pass. If the image width is less than or equal to 512 pixels, the $16 \times 16$ processes files in one pass, else it processes files in two passes. The $32 \times 32$ requires a pass for each 160 -pixel-wide strip. The last strip is not necessarily 160 pixels wide. For example, an image that is 350 pixels wide would require two 160 -pixel-wide strips and a third 30-pixel-wide strip. All strips are pasted together at the end of the procedure. As the files are processed, dots are displayed. Each continuous row of dots represents a strip. Lossy color procedures process $Y$ strips first, $C_{b}$ strips next, and $C_{r}$ strips last. The default lossy mode is $8 \times 8$.

Set Menu Color provides four menu color options: (a) Red, (b) Green, (c) Blue, and (d) Black and White.

Make Palette allows the user to create an RGB palette. The red, green, and blue intensities should be entered in ascending order. Once entered, the intensities are scrambled into an RGB palette. 


\section{Appendix C "Image View" Software User's Guide}

\section{File}

Load Series File uses information from a file to display a sequential set of images. The file format is as follows:

number_of_images mode1 palette_name1 image_name1 $x 1$ y1 mode2 ...

The modes are abbreviated as follows: $1=320 \times 200,2=640 \times 480$, $3=800 \times 600,4=1024 \times 768$. This file must be an ASCII file; if WP is used to generate this file, use CNTL F5 to save the file as ASCII text. Any combination of RGB and/or grayscale images may be displayed. Once a series is loaded, it is stored in global memory; therefore, the series does not need to be reloaded to perform "View" selections.

Quit ends the use of "Image View."

\section{View}

Show Red shows only the red intensities. Show Green shows only the green intensities. Show Blue shows only the blue intensities. Show Bit Plane 1-7 masks out all bits except the requested one. If the bit is present in a pixel, white will be displayed, else black will be displayed. Display Full Image redisplays the original series.

\section{Options}

Set Menu Color provides four menu color options: (a) Red, (b) Green, (c) Blue, and (d) Black and White. 


\section{Appendix D \\ Source Code for Individual Programs}

ENTROPY.BAS

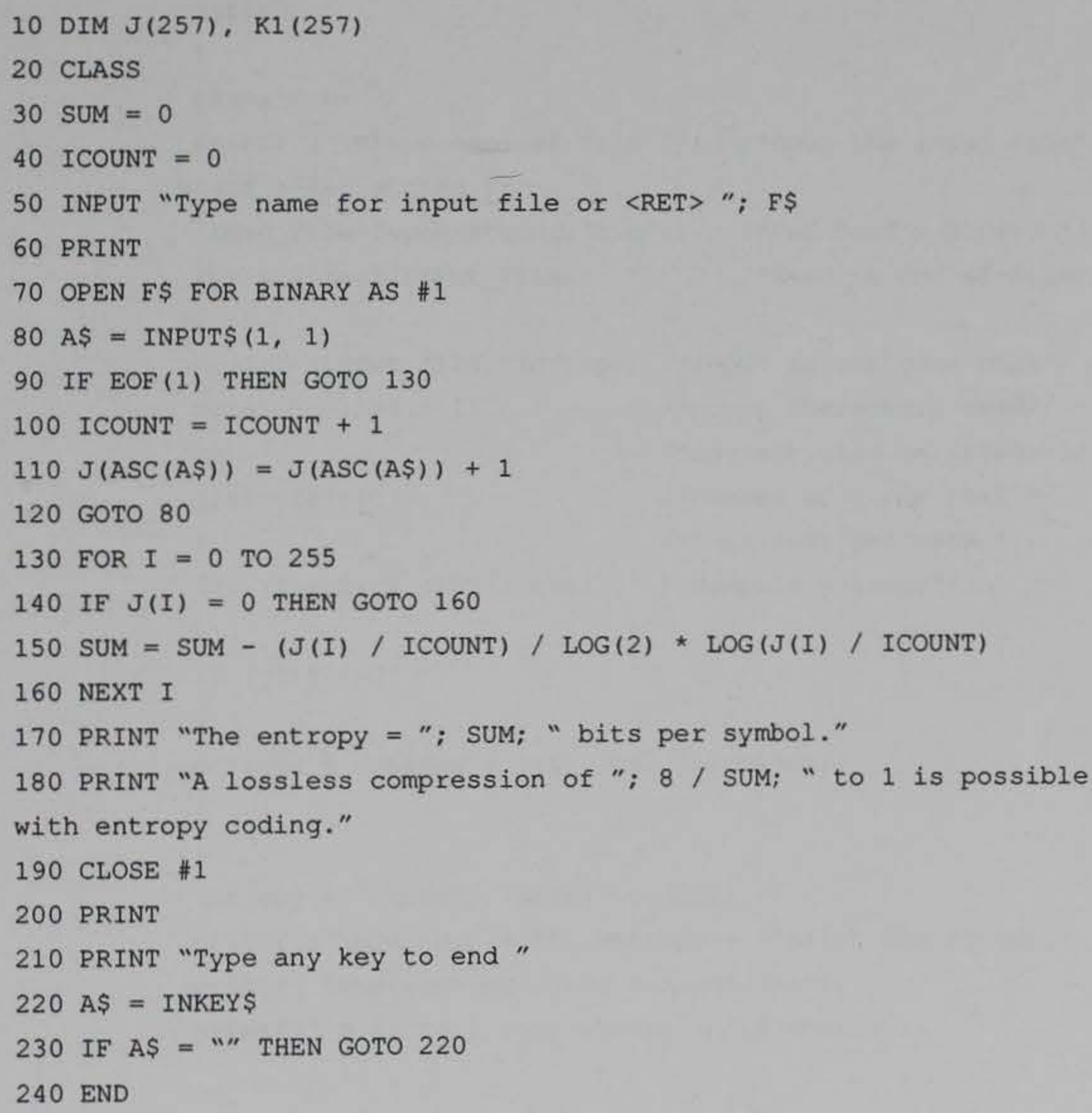




\section{REM files}

$30 \mathrm{n}=1$

40 CLASS

50 INPUT "Type filename \#1 "; $F \$$

60 INPUT "Type filename \#2 "; G\$

70 OPEN F\$ FOR BINARY AS \#1

80 OPEN GS FOR BINARY AS \#2

90 AS $=\operatorname{INPUT} \$(1,1)$

$100 \mathrm{~B} \$=\operatorname{INPUT} \$(1,2)$

110 IF EOF (1) THEN GOTO 1000

120 SUM $=\operatorname{SUM}+(A S C(A \$)-A S C(B \$)) \wedge 2$

$130 \mathrm{n}=\mathrm{n}+1$

140 GOTO 90

1000 SUM $=$ SUM $/ \mathrm{n}$

1010 PRINT "The mean square error $=$ "; SUM 


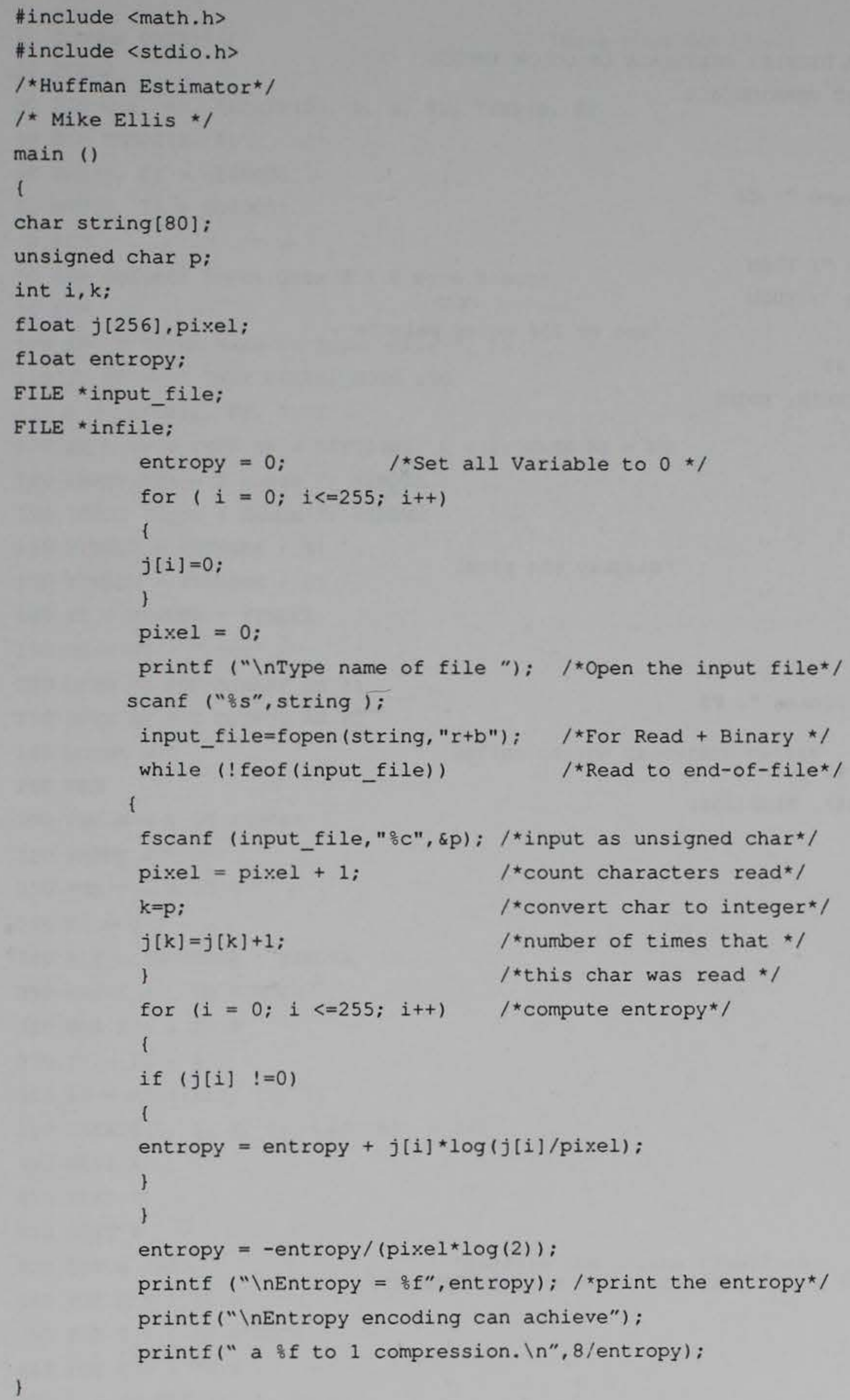


IMAGE. BAS

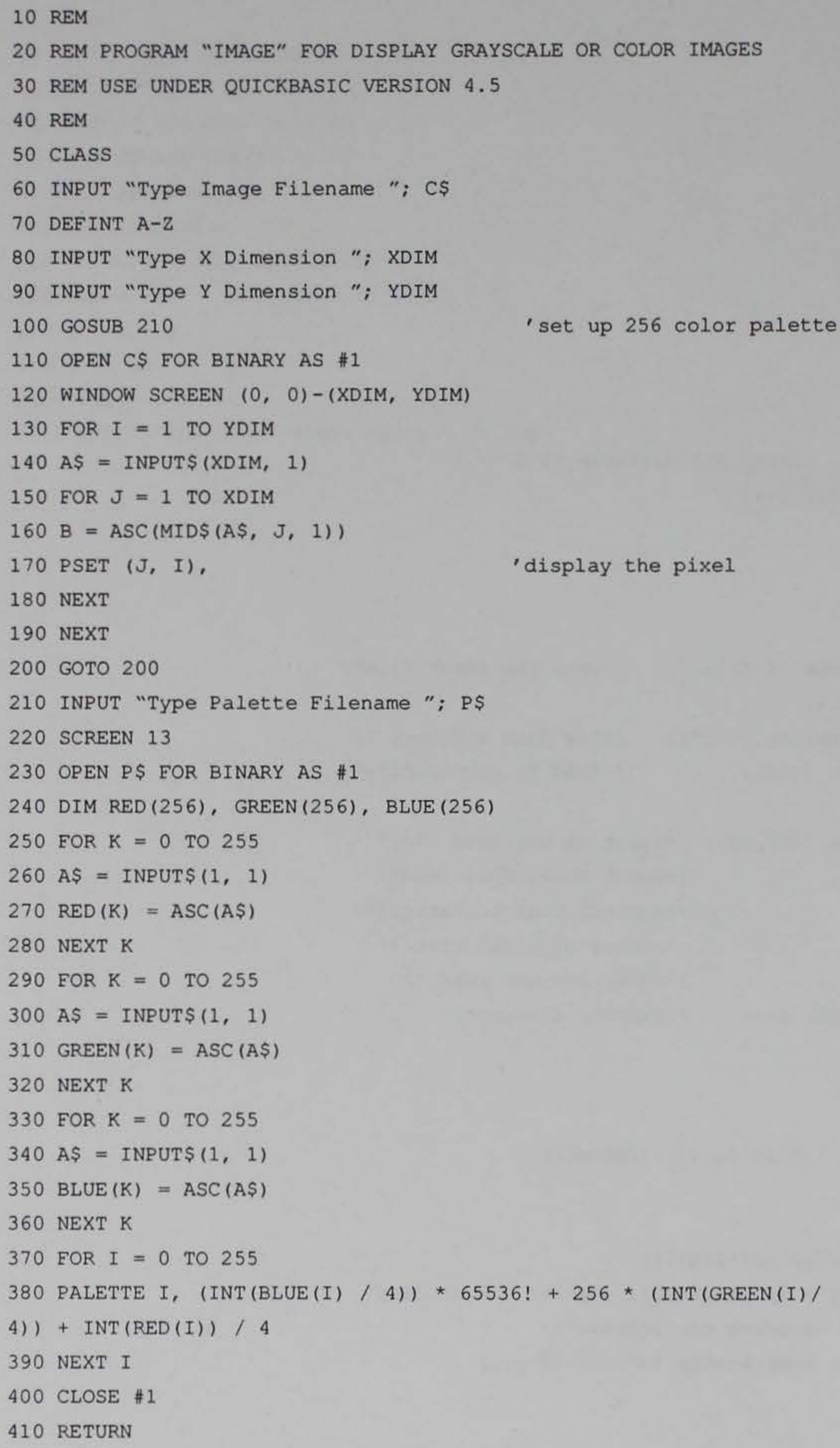




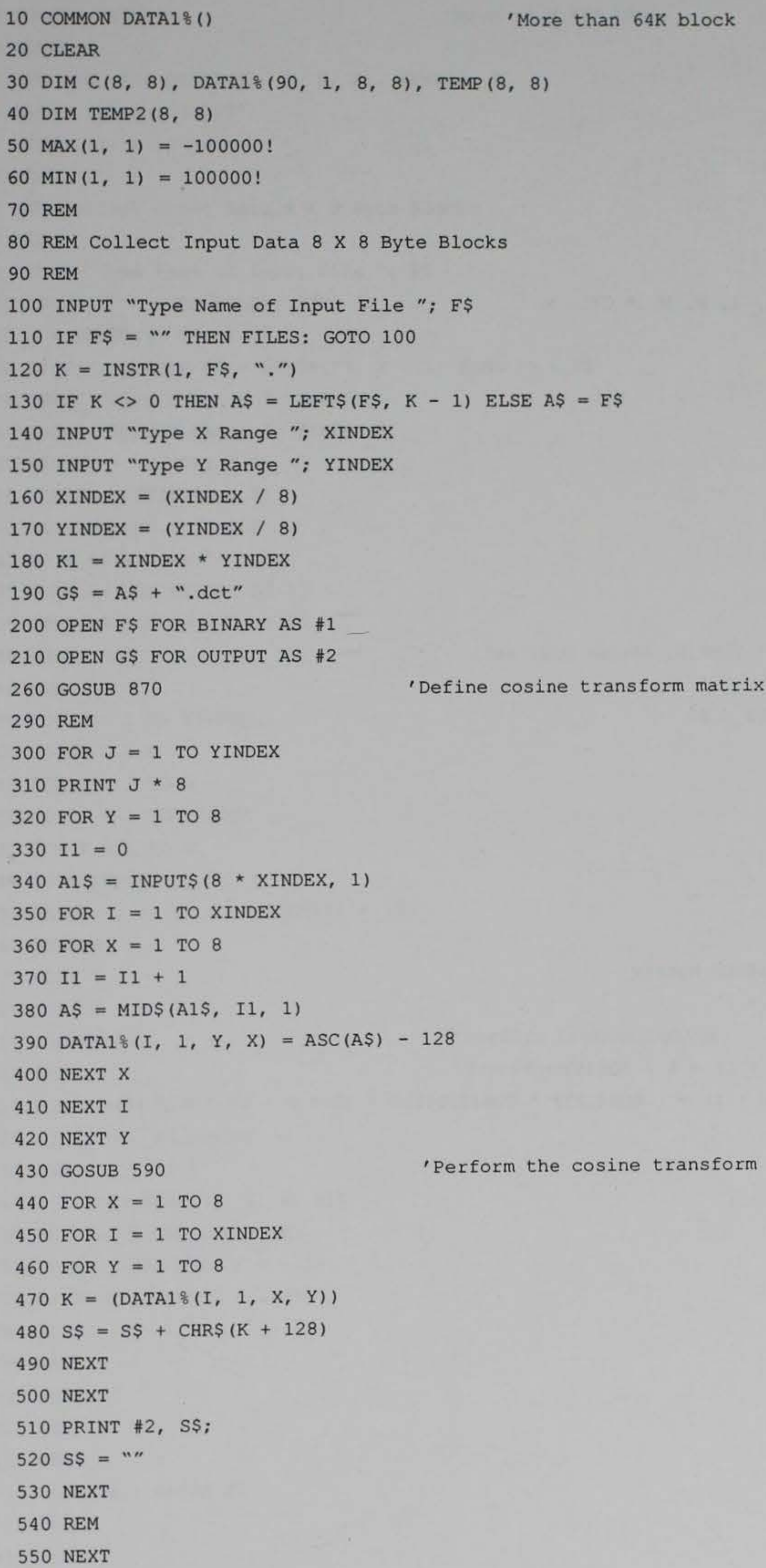




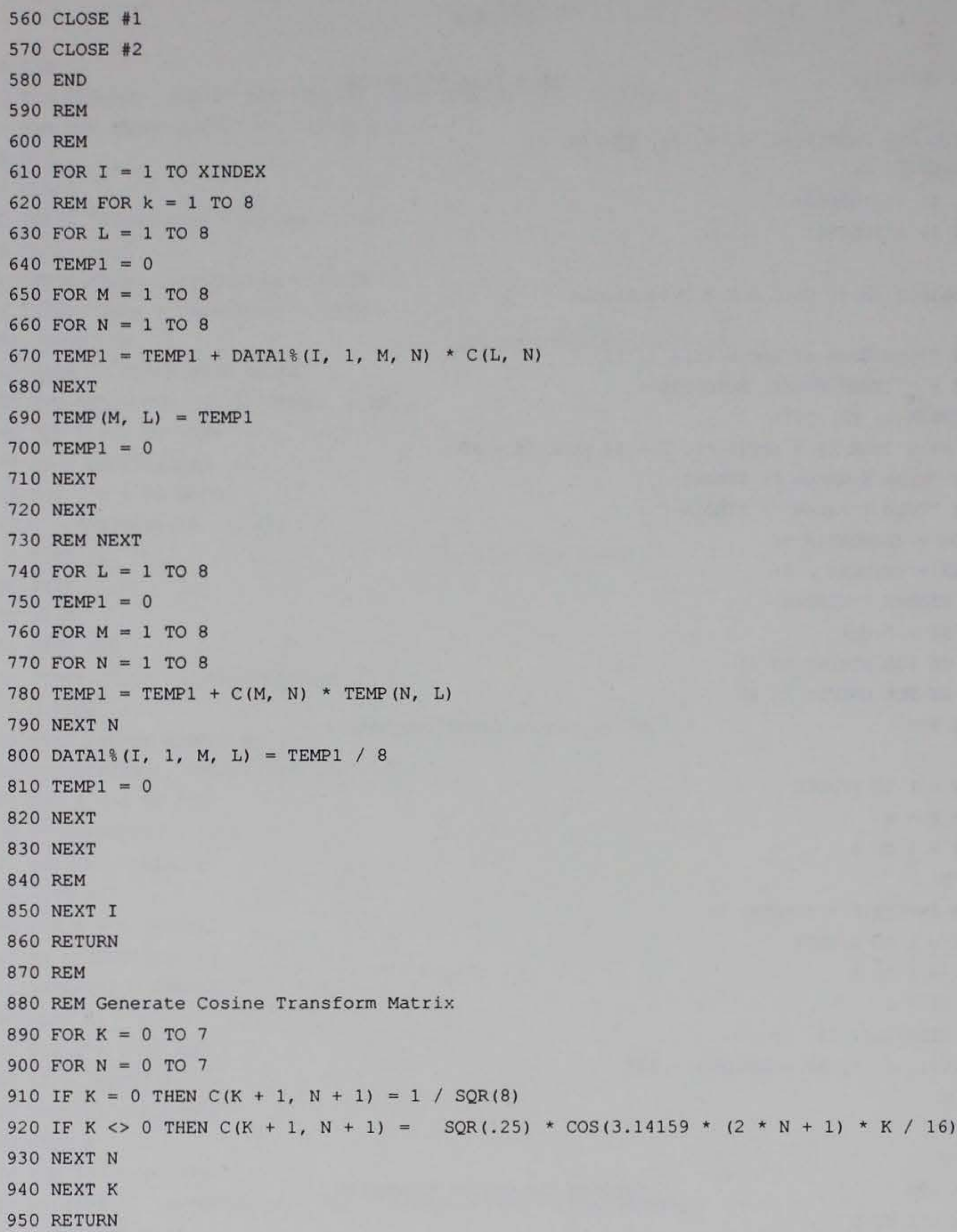




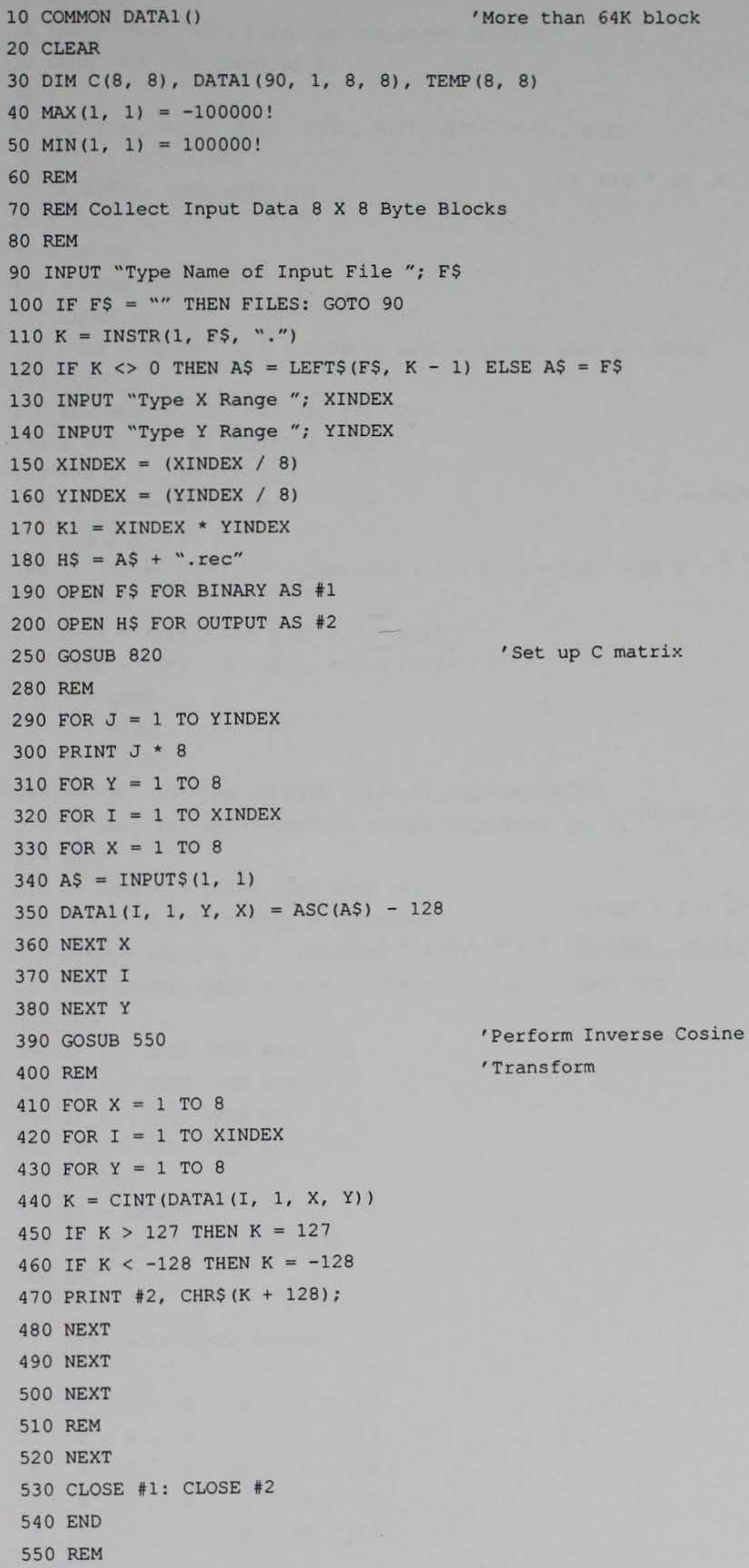




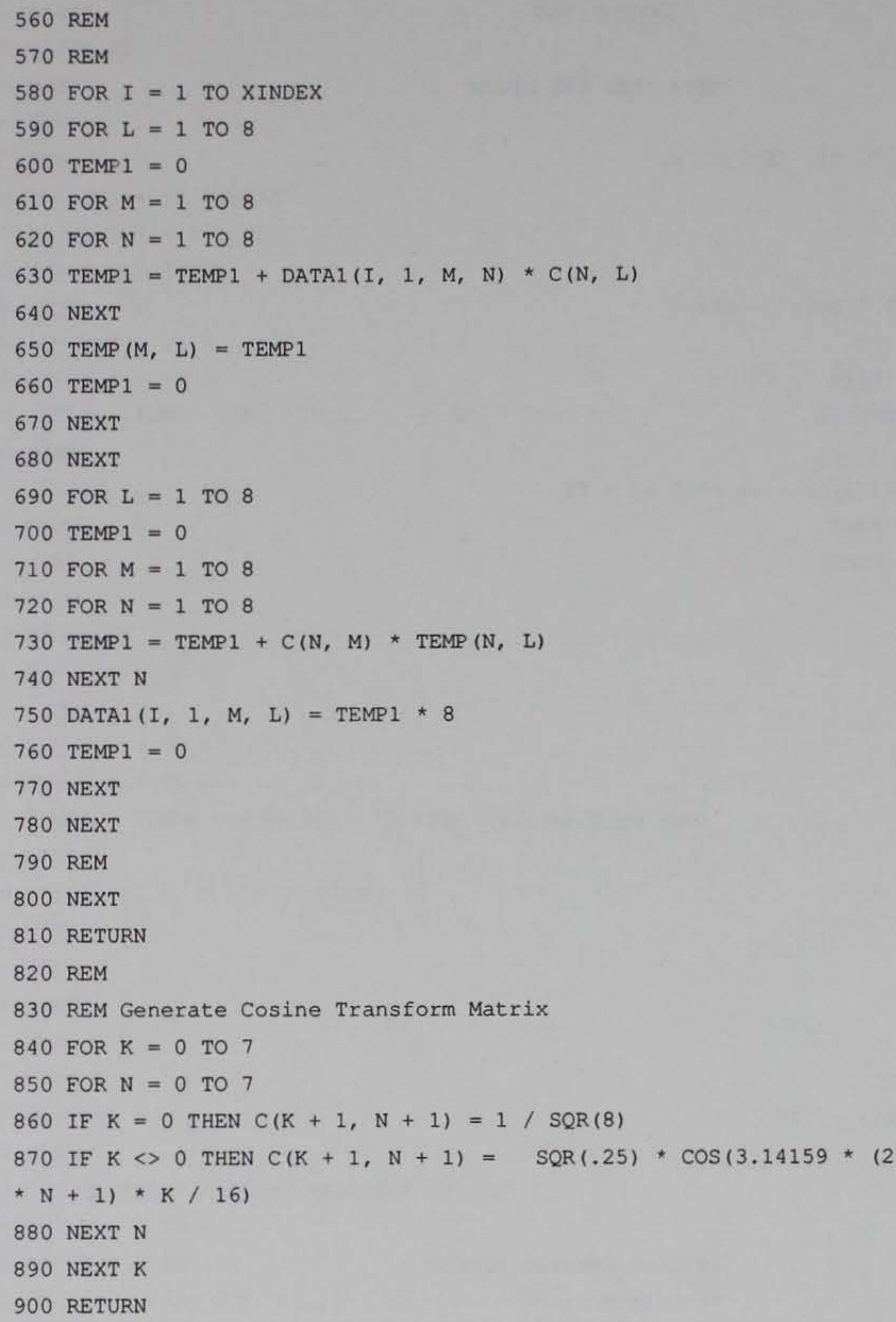




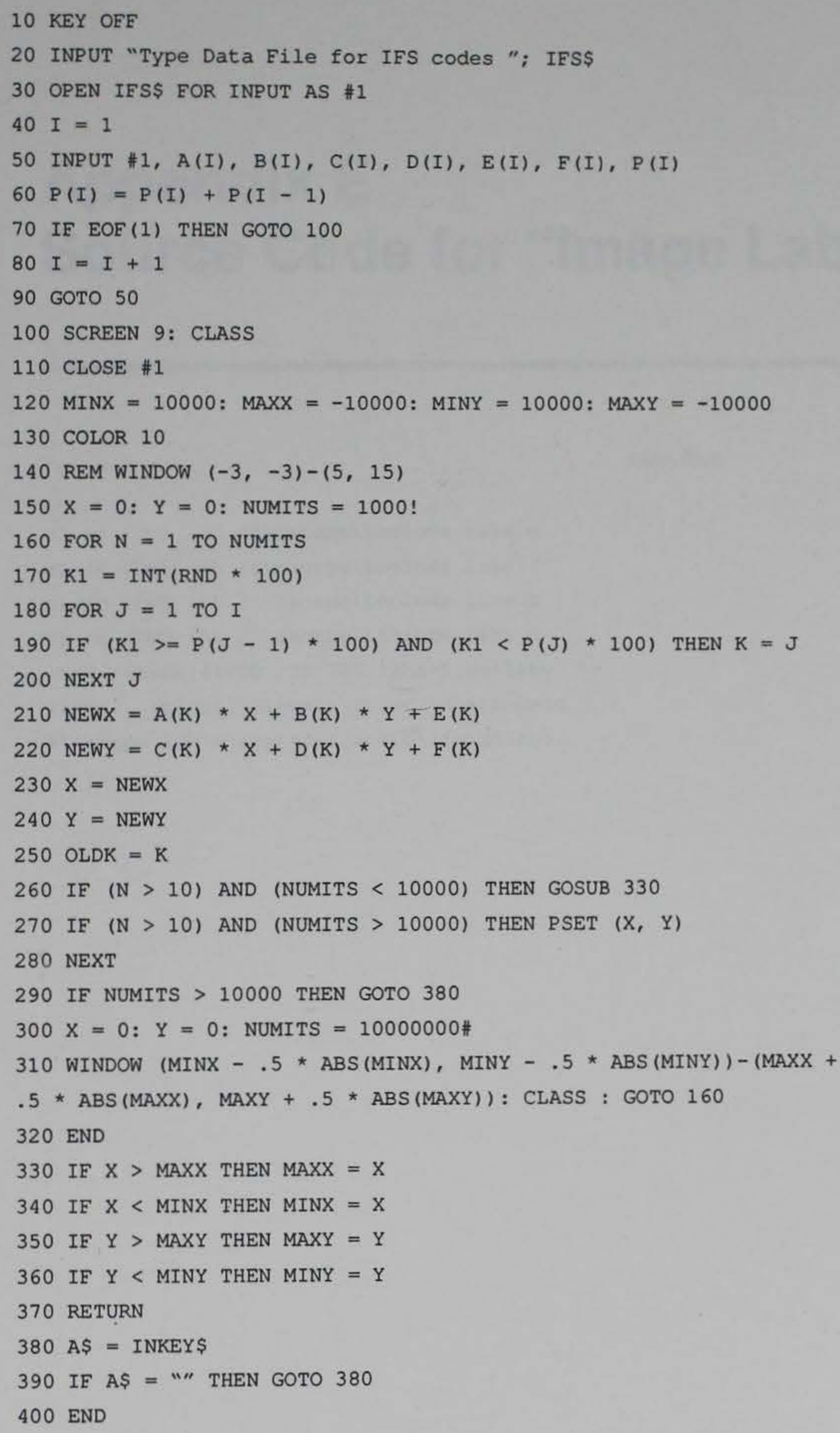




\title{
Appendix E Source Code for "Image Lab" Software
}

\begin{abstract}
cl /C /DM5 /AL /I \cscapelinclude labs.c
cl /c /DM5 /AL /I lcscapelinclude labx,c

cl /c /DM5 /AL /I \cscapelinclude laby.c cl /C /DM5 /AL /I \cscapelinclude labz.c link/stack:44000/SE: 300 labs+labx+laby +1 abz, , , Iglobal \global+\standard $\backslash$ standard + \cscape $\backslash$ lib $\backslash$ m1lcscap+|cscape $\backslash l i b \backslash m 1 l o w l$;
\end{abstract}




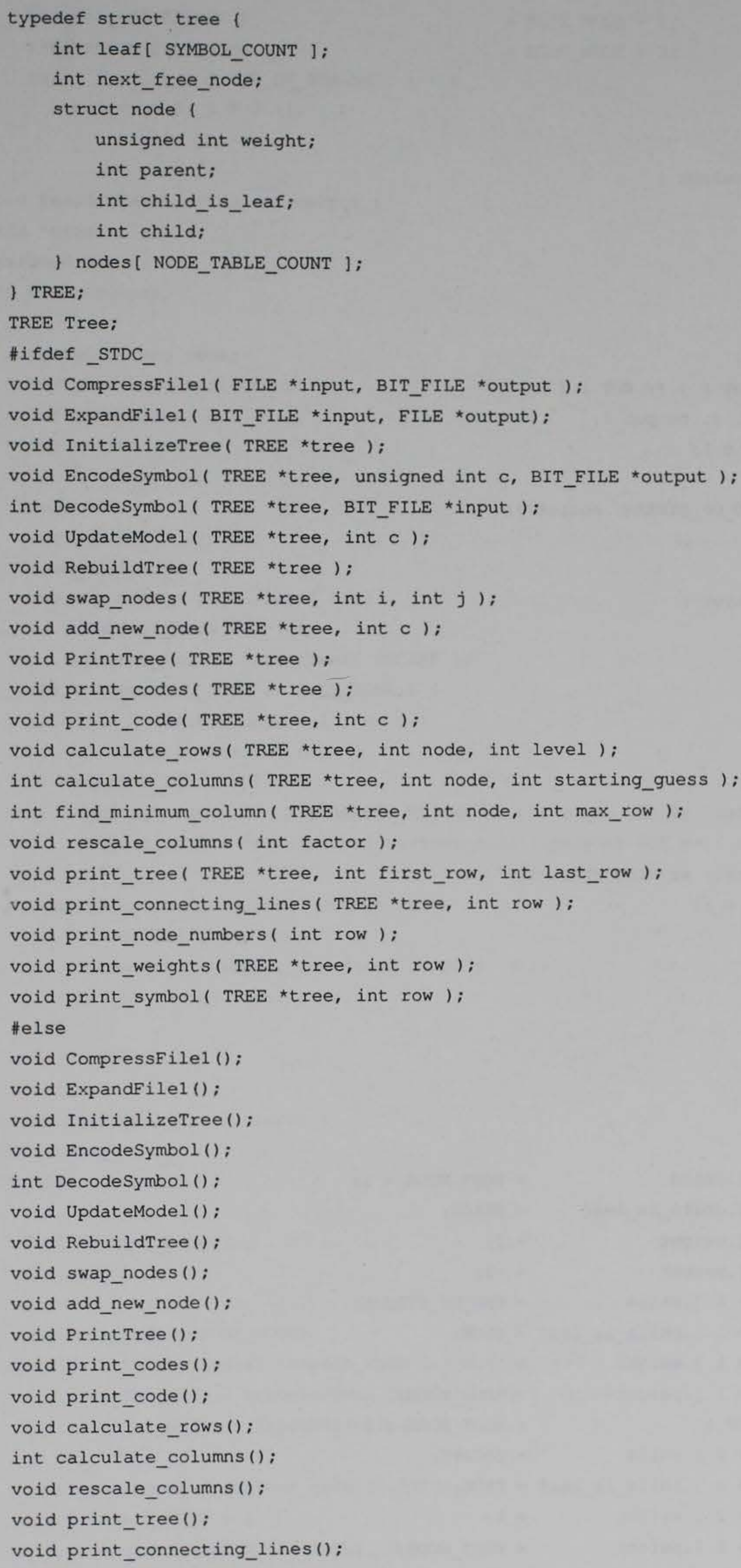




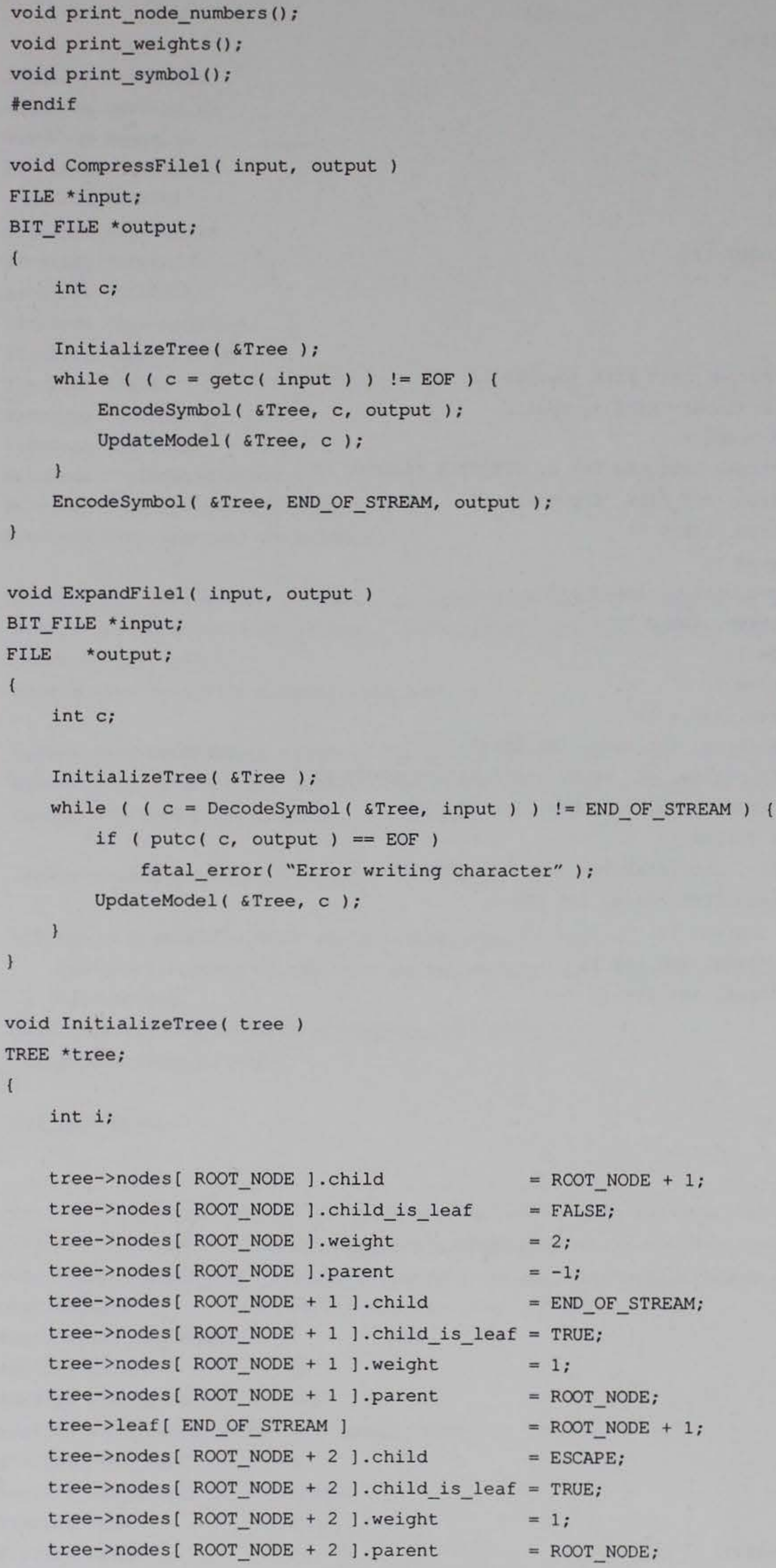




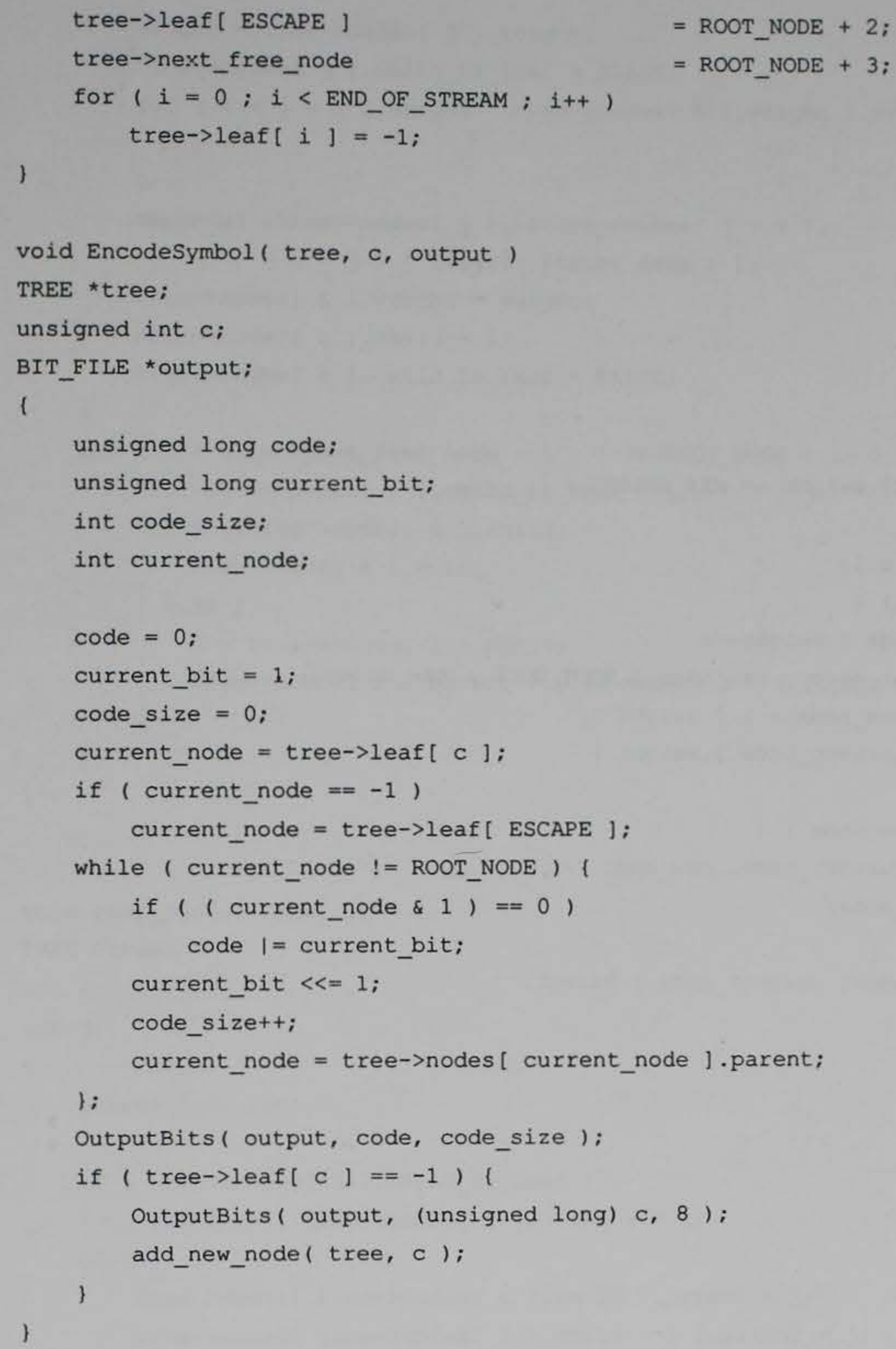




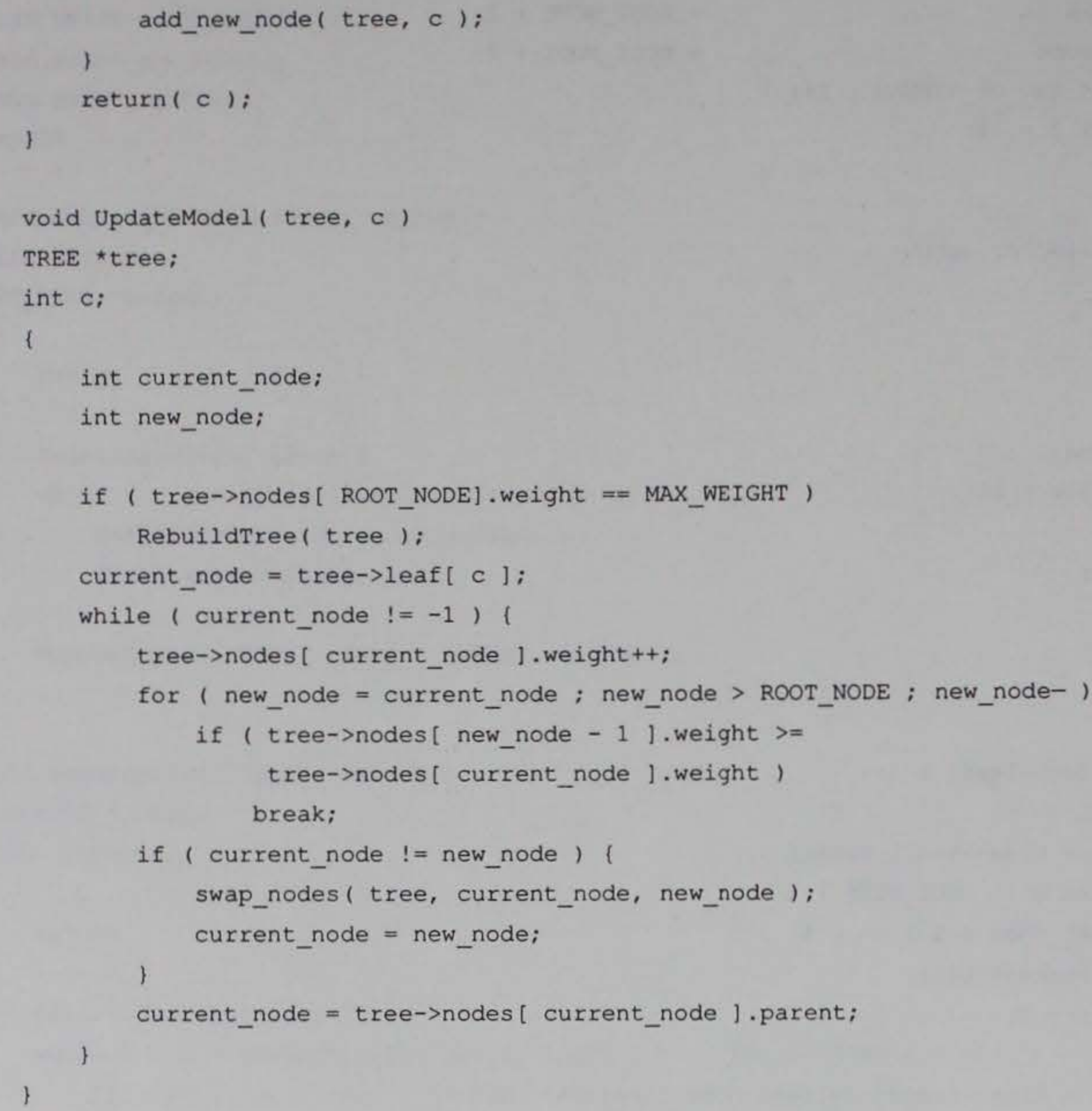


int $c$;

i

int lightest_node;

int new_node;

int zero_weight_node;

lightest_node $=$ tree->next_free_node -1 ;

new_node $=$ tree->next_free_node;

zero_weight_node $=$ tree->next_free_node +1 ;

tree->next_free_node $+=2$;

tree->nodes [ new_node ] $=$ tree->nodes [ lightest_node ];

tree->nodes [ new_node ] $\cdot$ parent $=1$ ightest_node;

tree->leaf [ tree->nodes [ new_node ].child ] = new_node;

tree->nodes [ lightest_node ].child = new_node;

tree->nodes [ lightest_node ].child_is_leaf = FALSE;

tree->nodes [ zero_weight_node ].child $=\mathrm{c}$;

tree->nodes[ zero_weight_node ].child_is_leaf = TRUE;

tree->nodes [ zero_weight_node ].weight $\quad=0$;

tree->nodes[ zero_weight_node ].parent = lightest_node;

tree->leaf [ c ] = zero weight node;

struct row \{

int first_member;

int count;

\} rows [ 32$]$;

struct location

int row;

int next_member;

int column;

l positions[ NODE_TABLE_COUNT ];

void PrintTree ( tree )

TREE *tree;

1

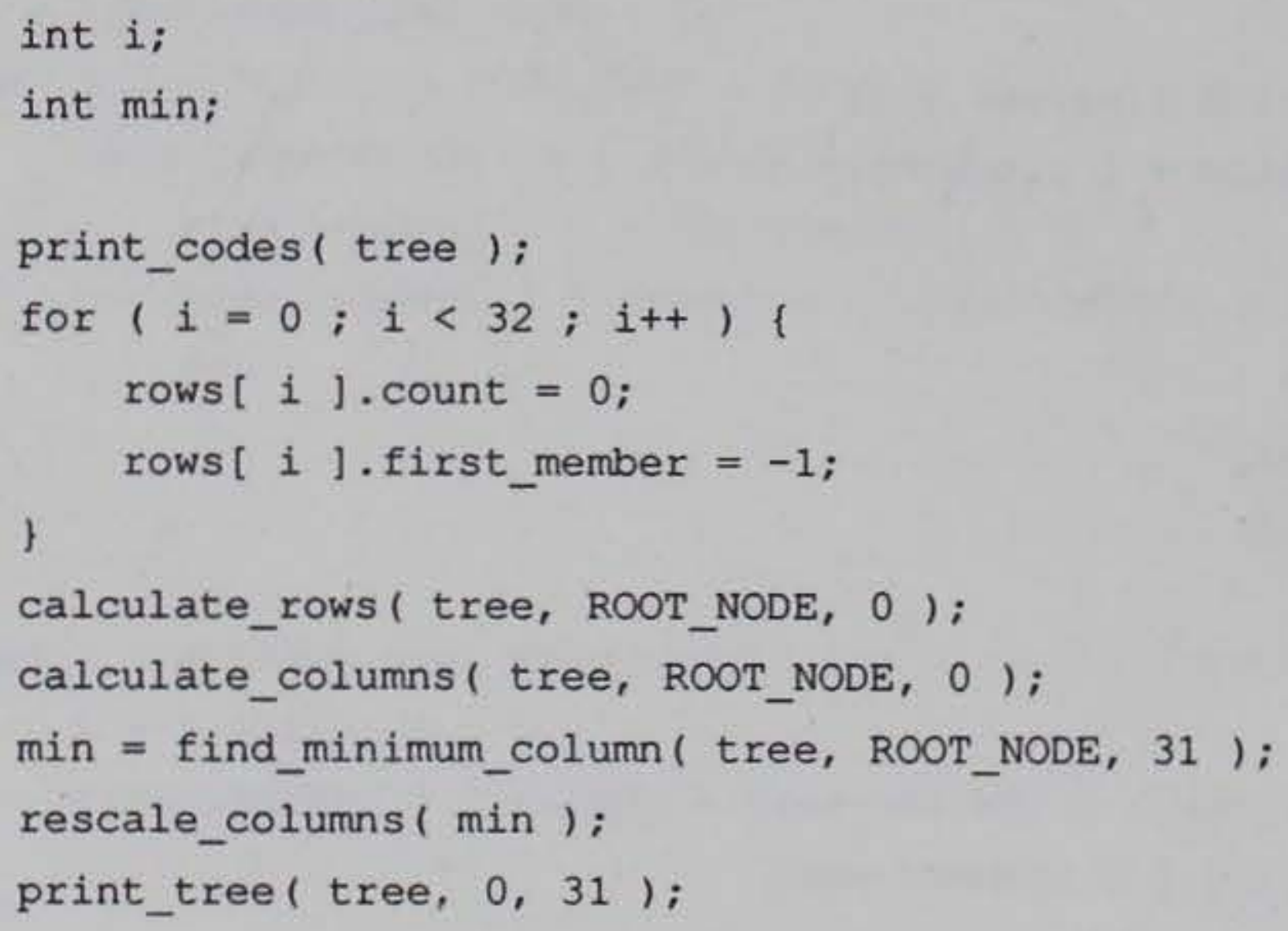




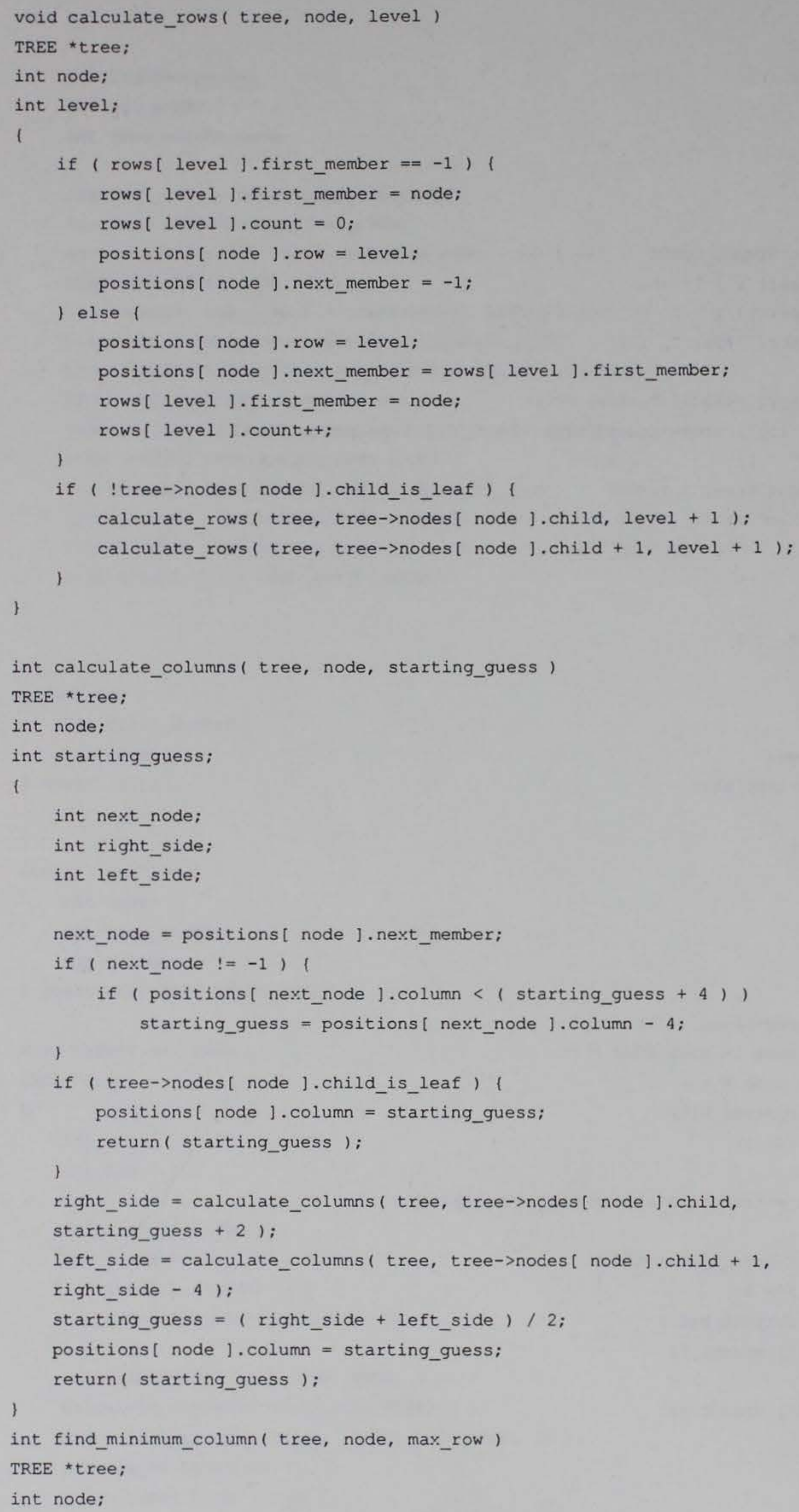




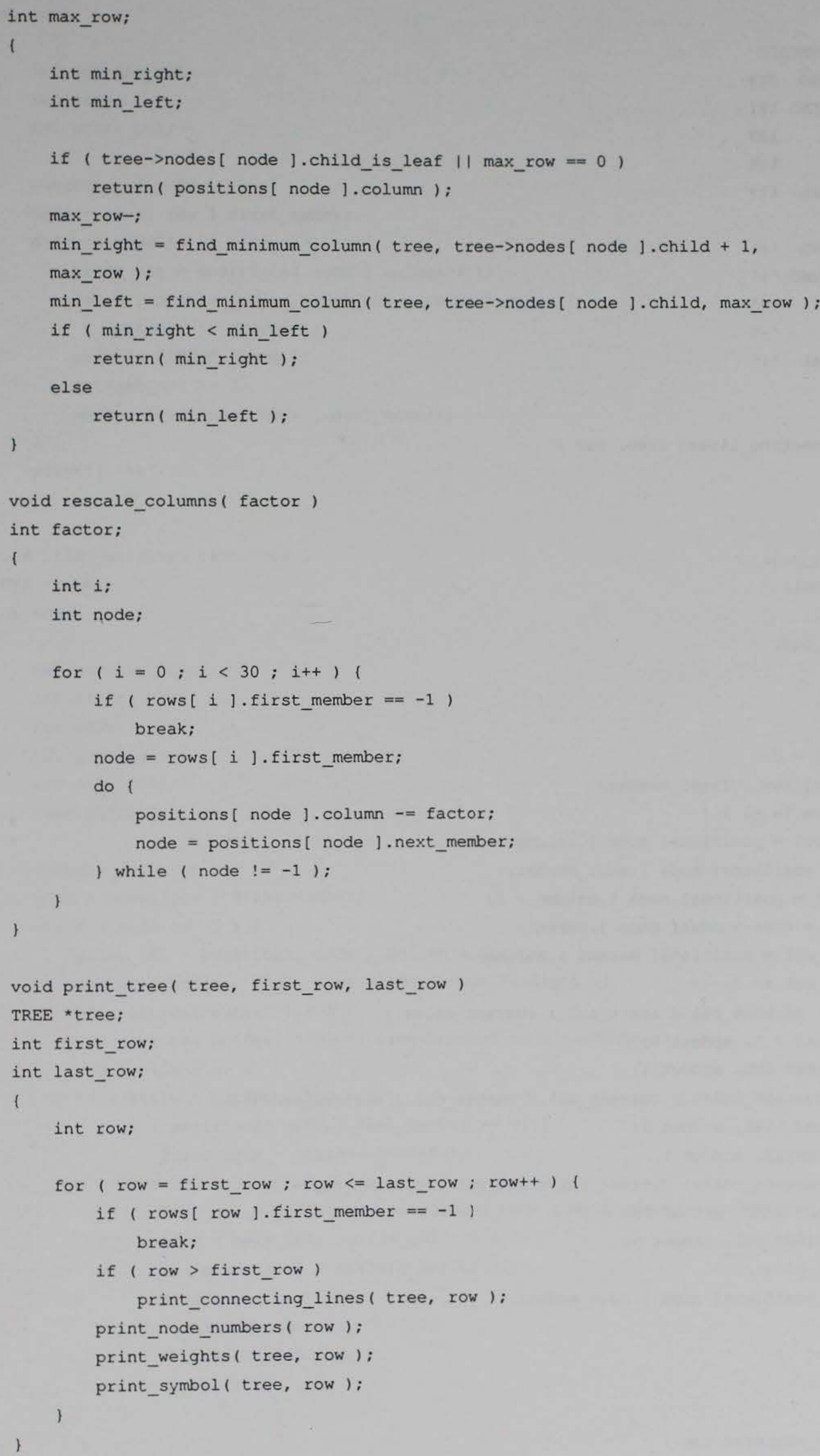




\section{\#ifndef ALPHANUMERIC \\ \#define LEFT_END 218 \\ \#define RIGHT_END 191 \\ \#define CENTER 193 \\ \#define LINE 196 \\ \#define VERTICAL 179 \\ \#else \\ \#define LEFT_END ' + ' \\ \#define RIGHT_END ' + ' \\ \#define CENTER ' + ' \\ \#define LINE ' -' \\ \#define VERTICAL ' $\mid$ ' \\ \#endif}

void print_connecting_lines ( tree, row )

TREE *tree;

int row;

\{

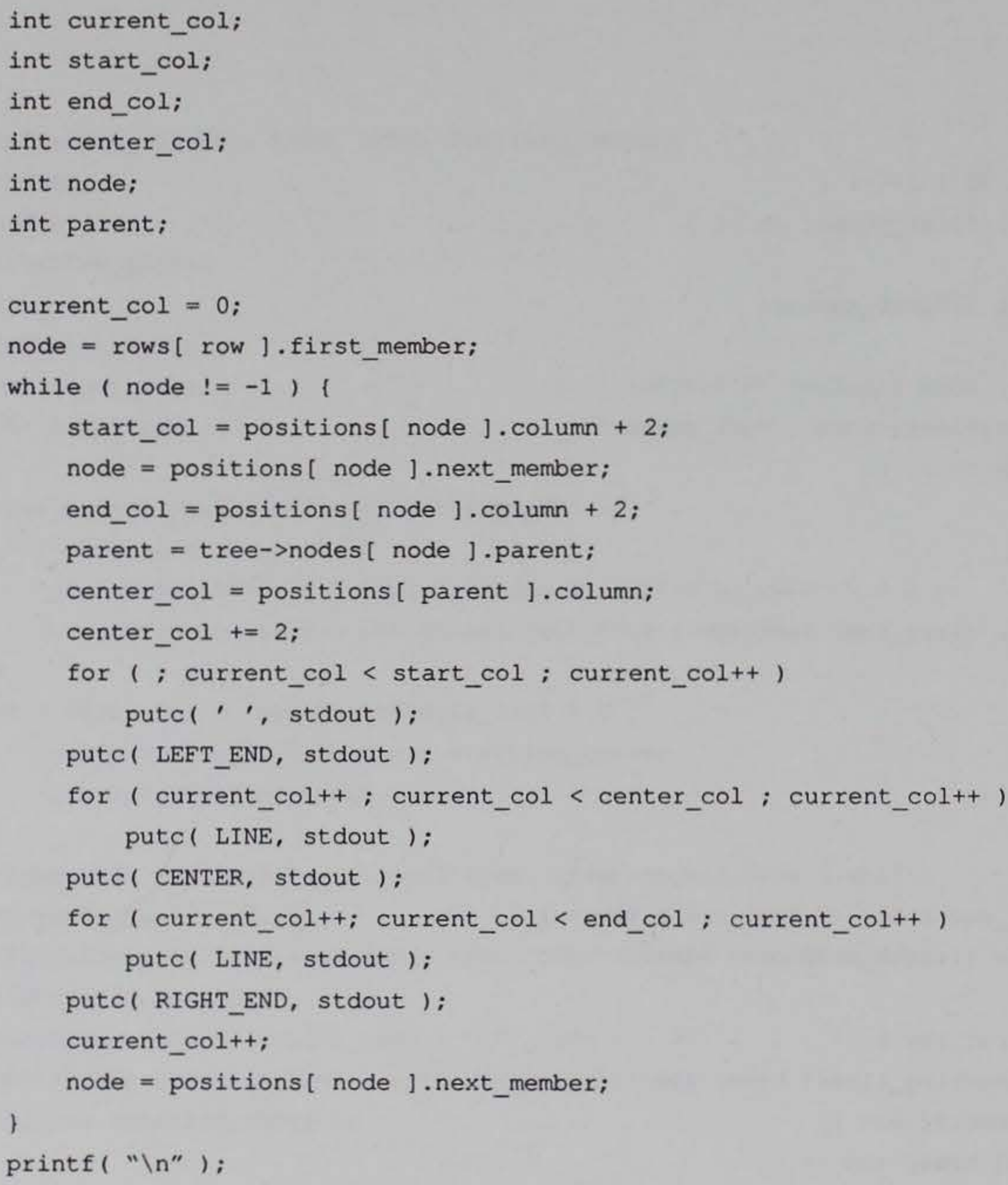




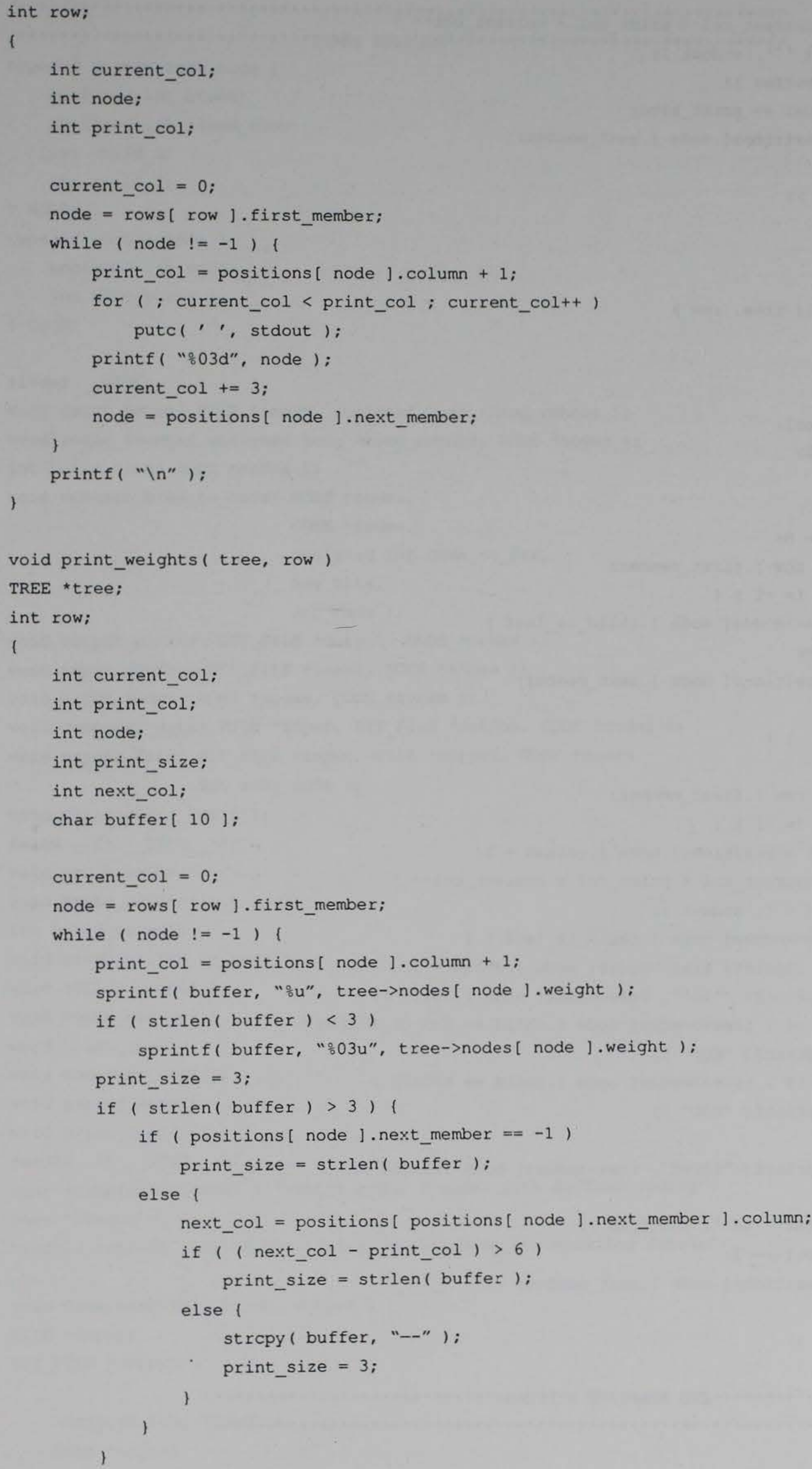




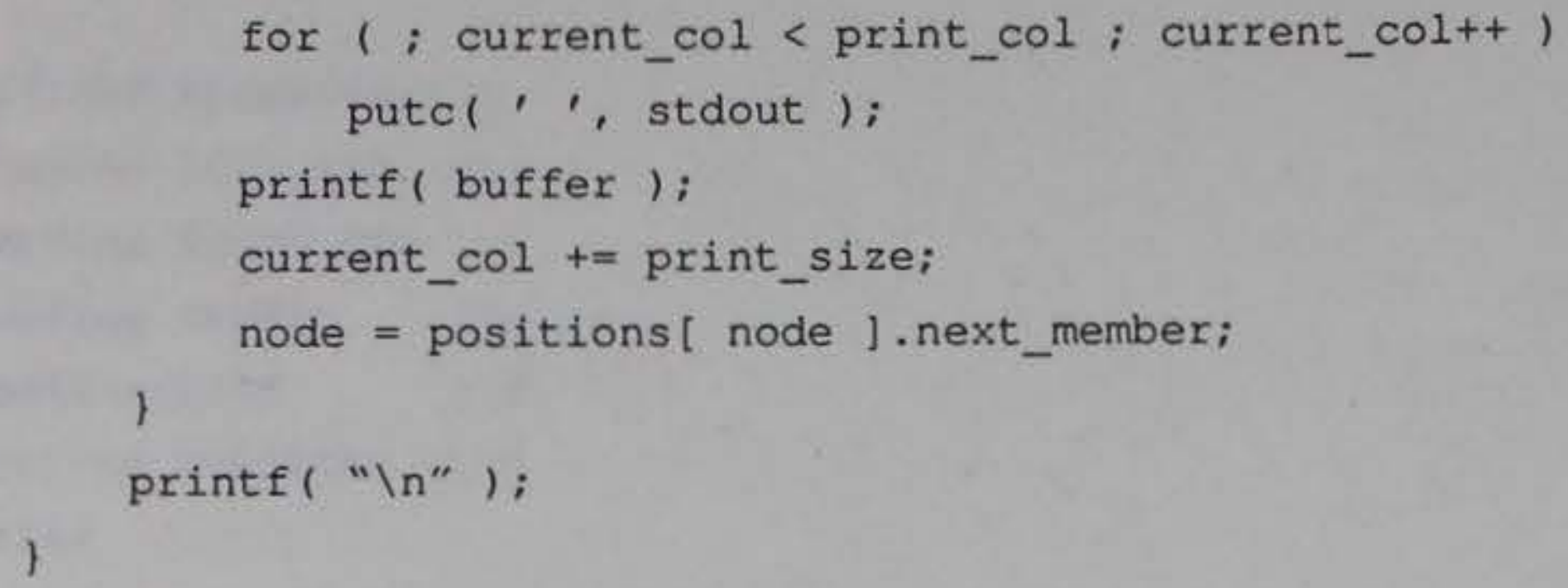




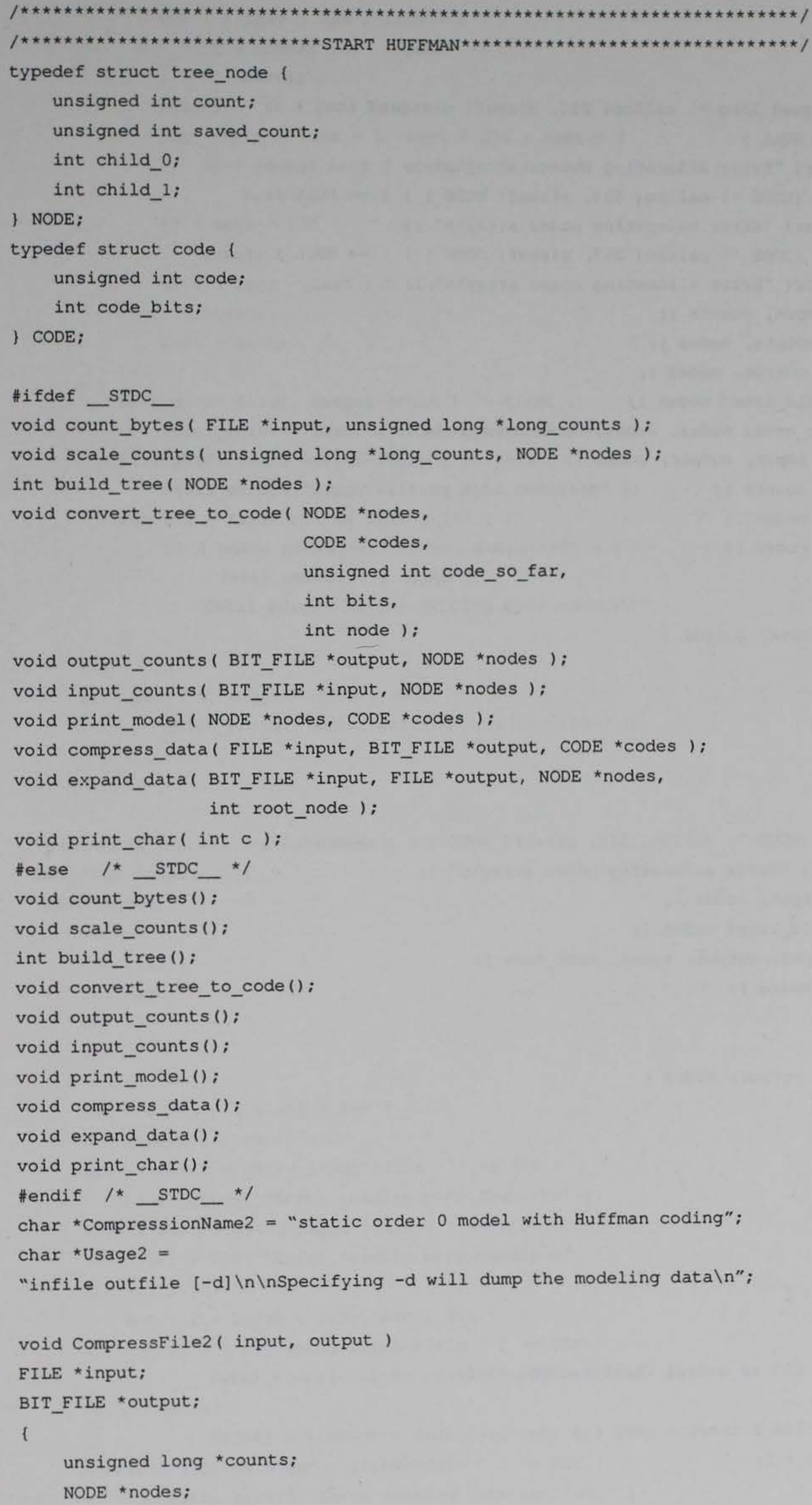




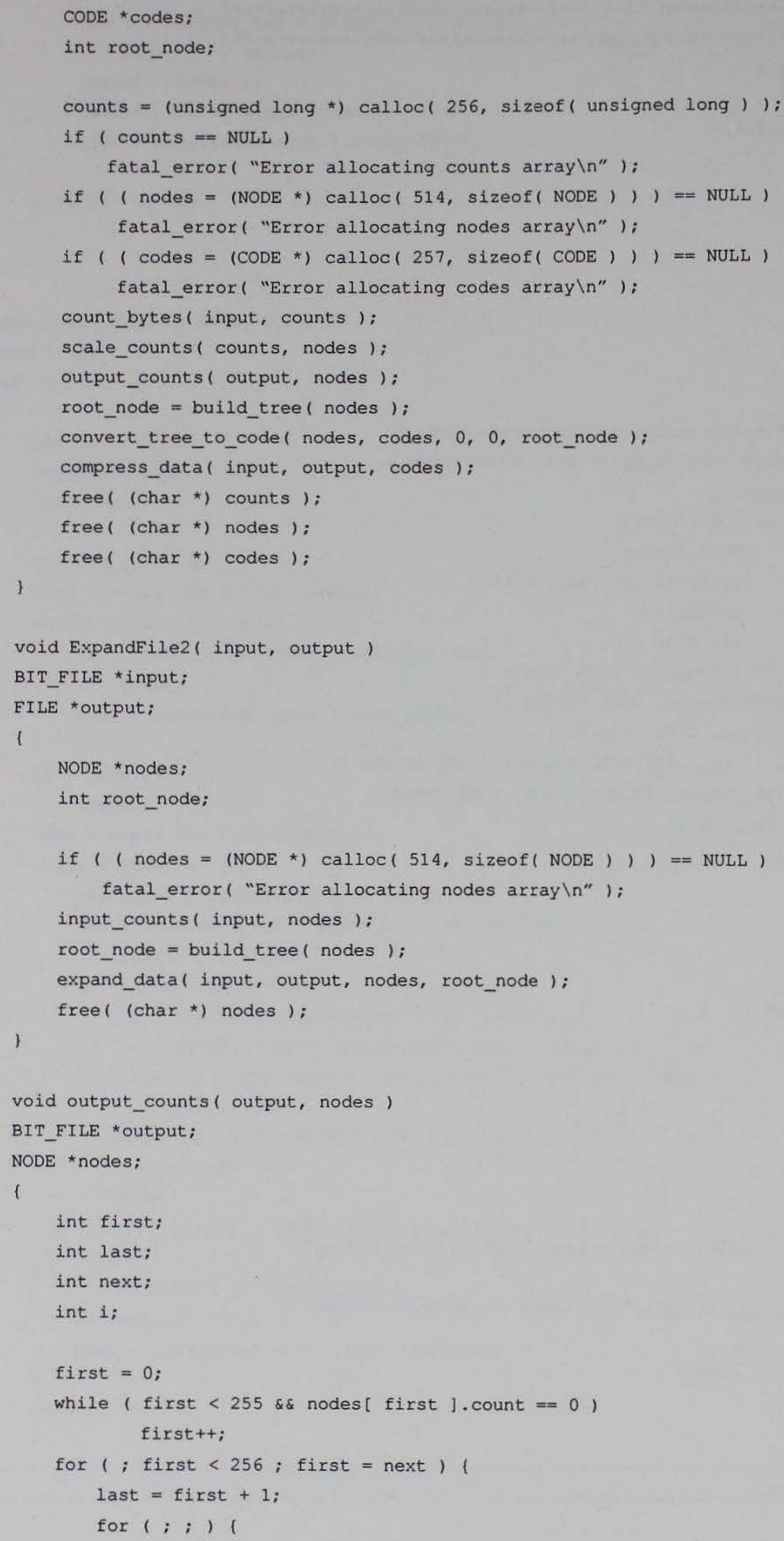




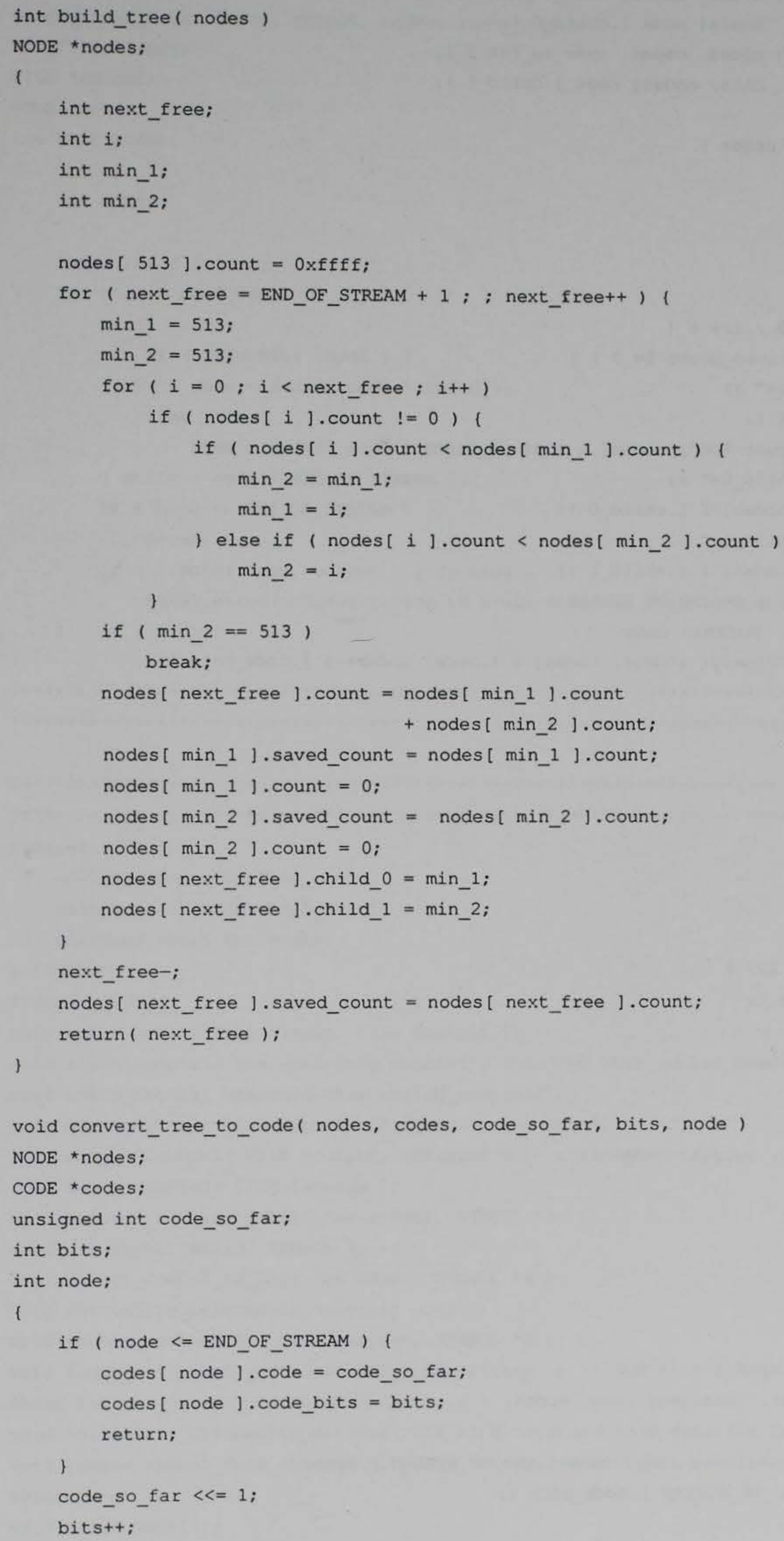




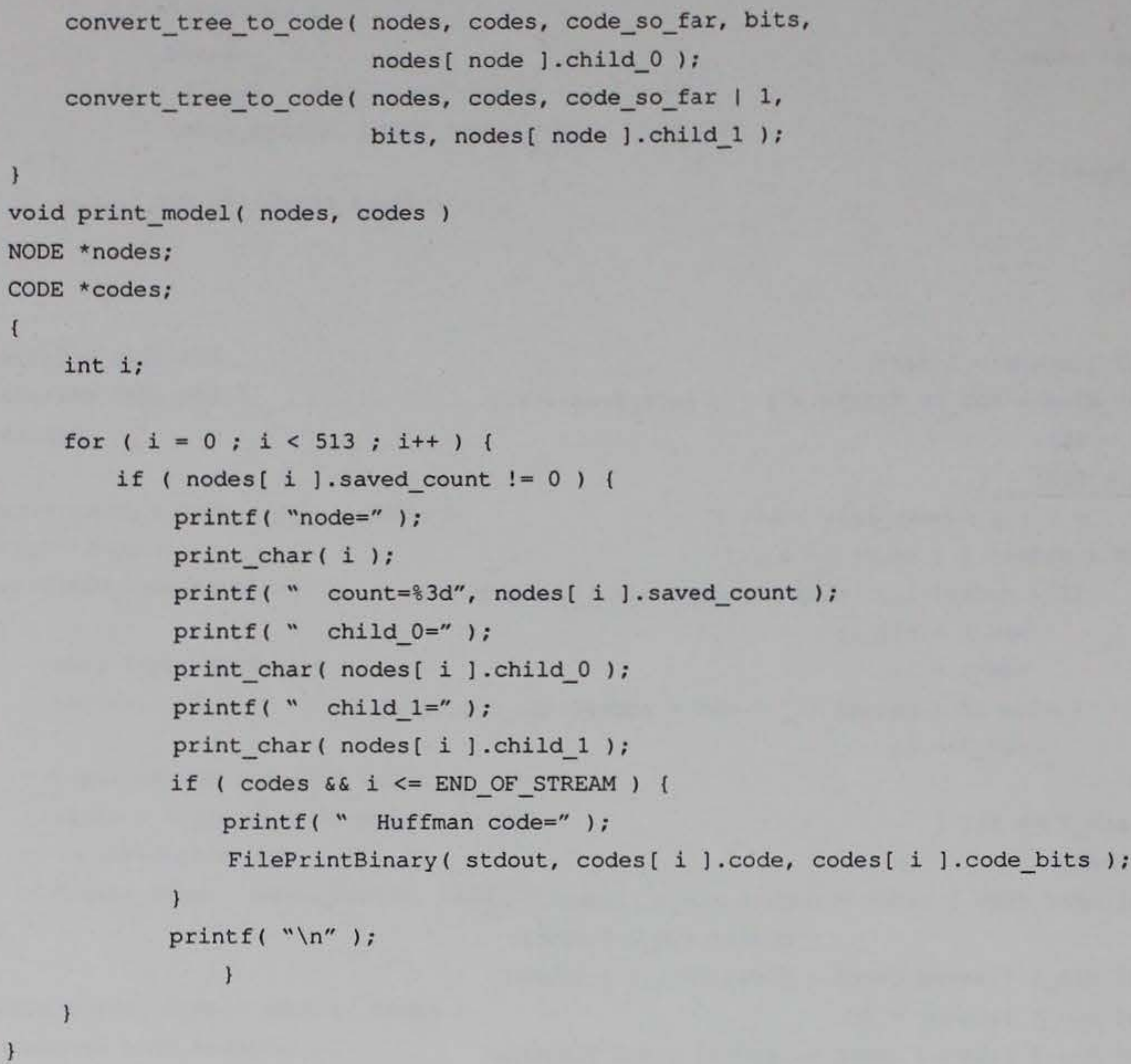




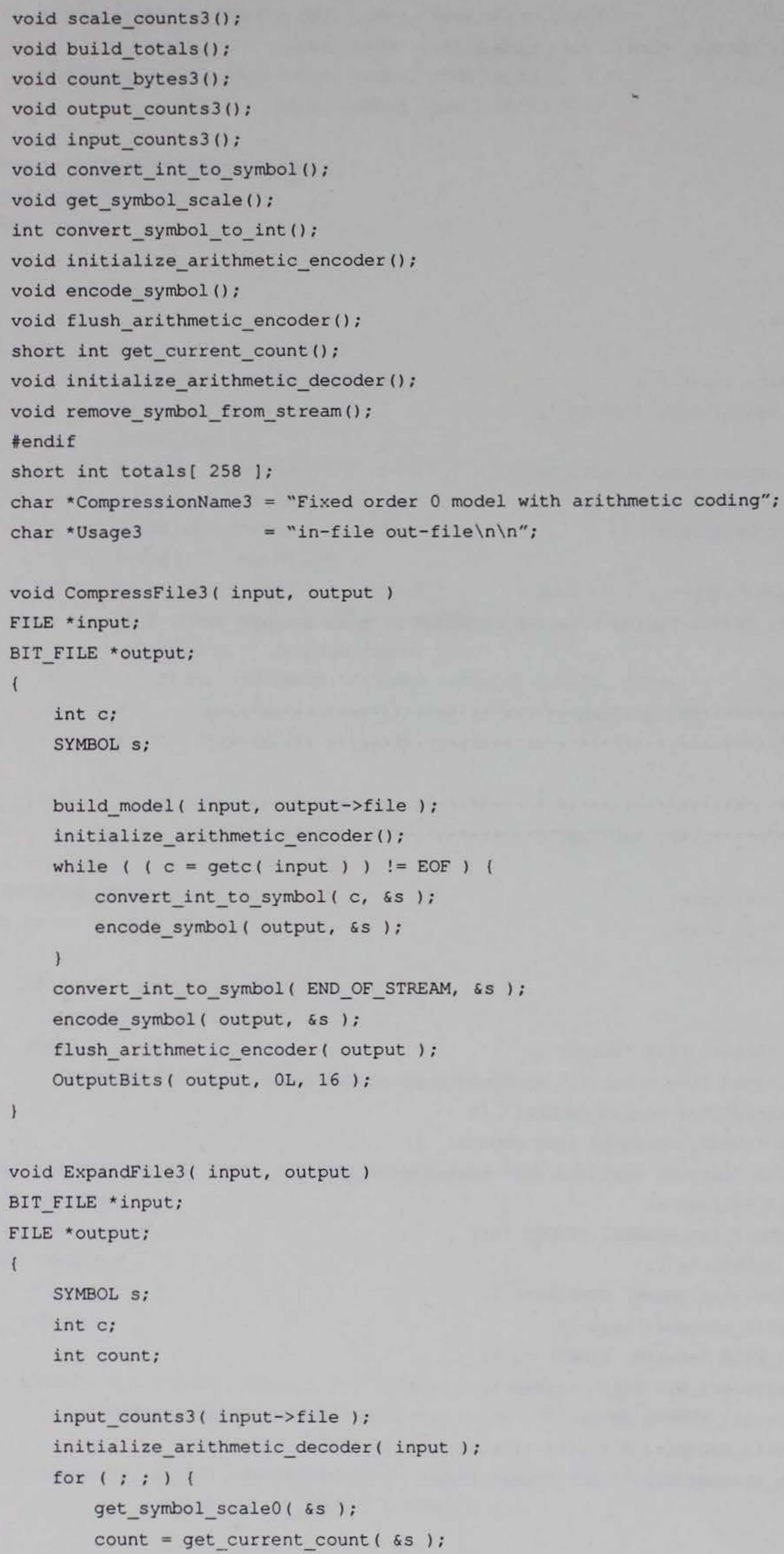




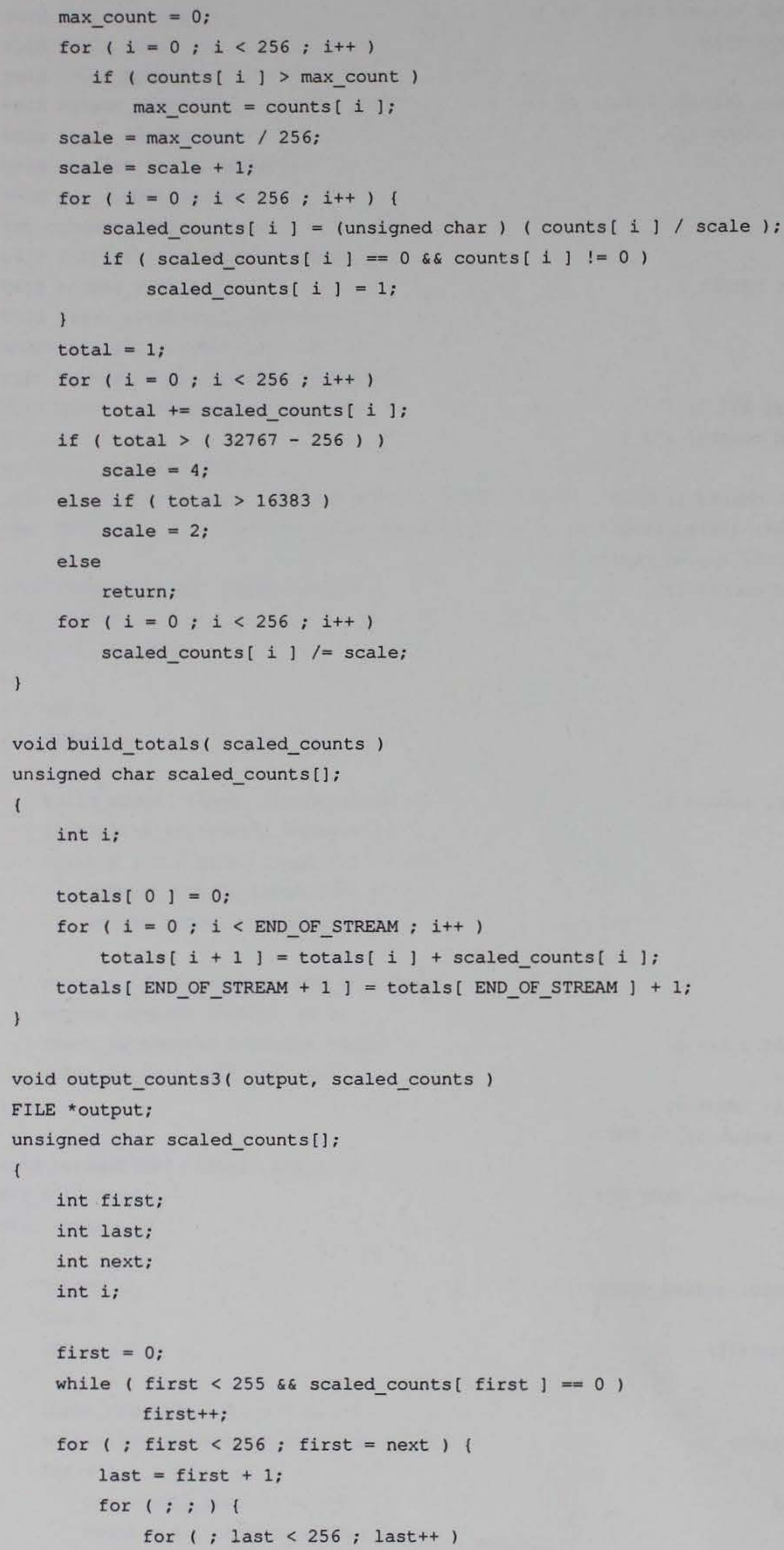




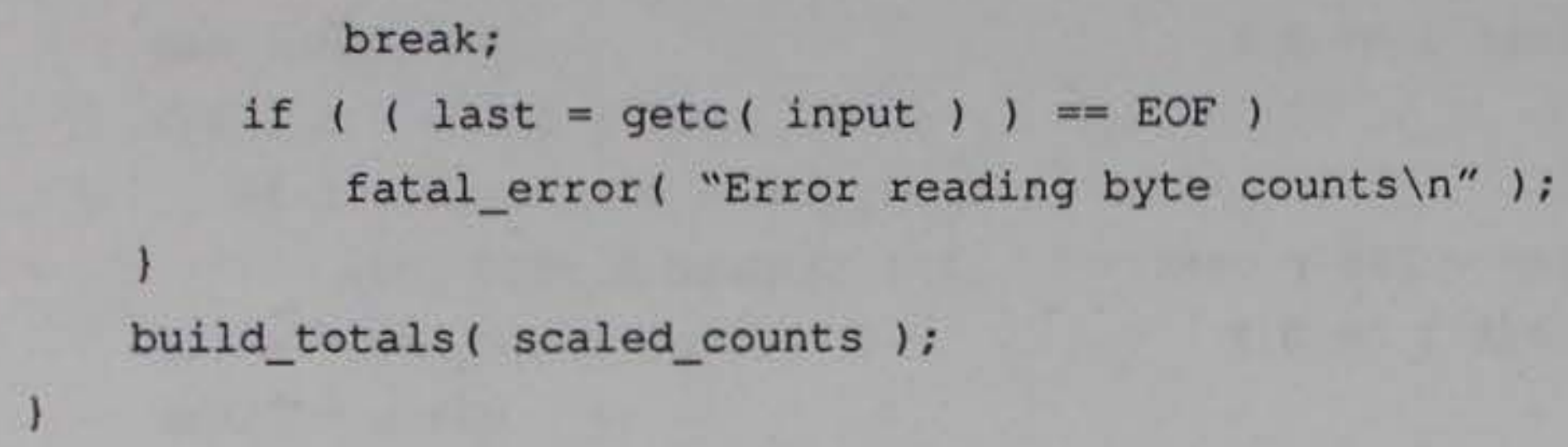




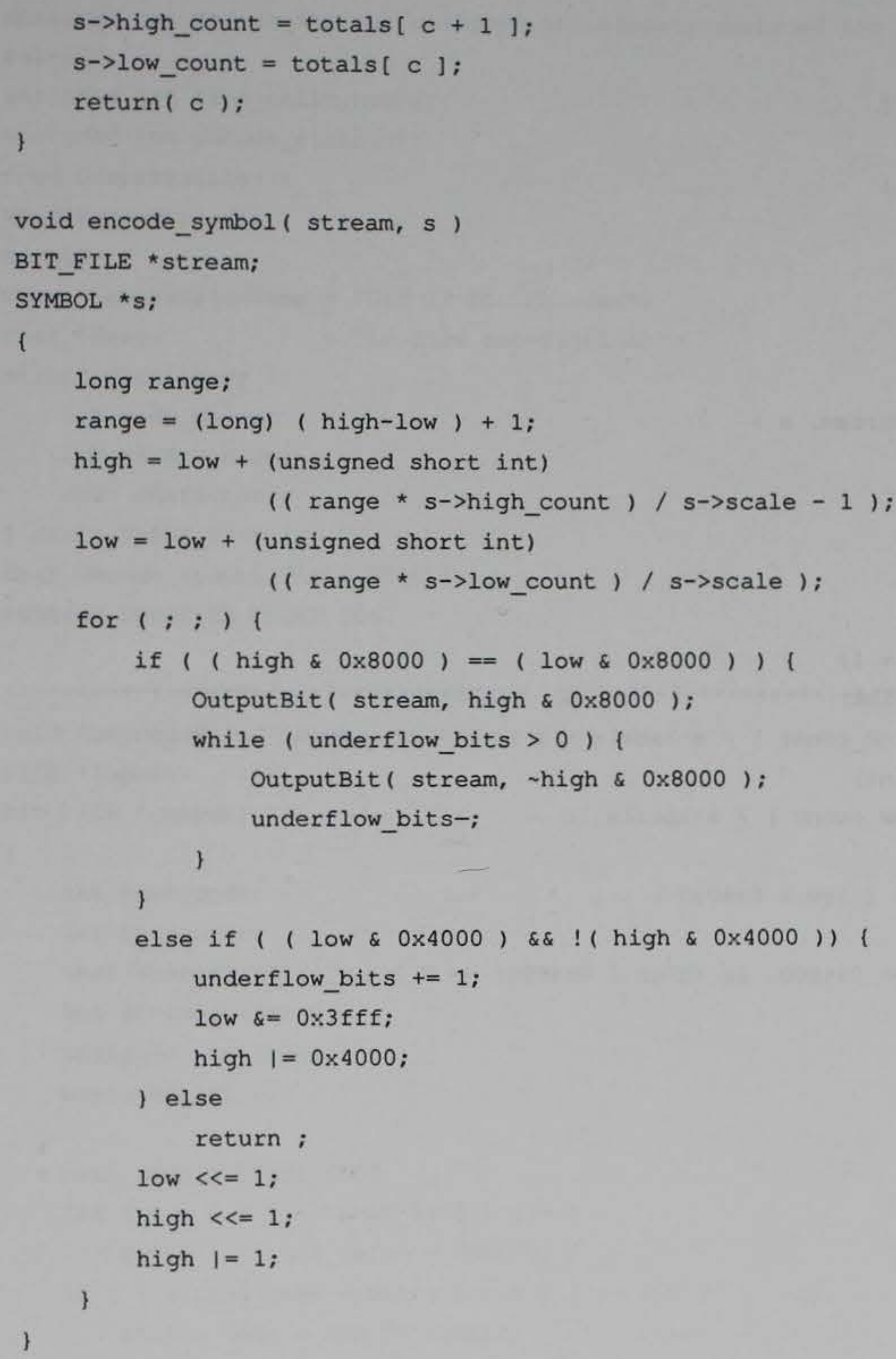



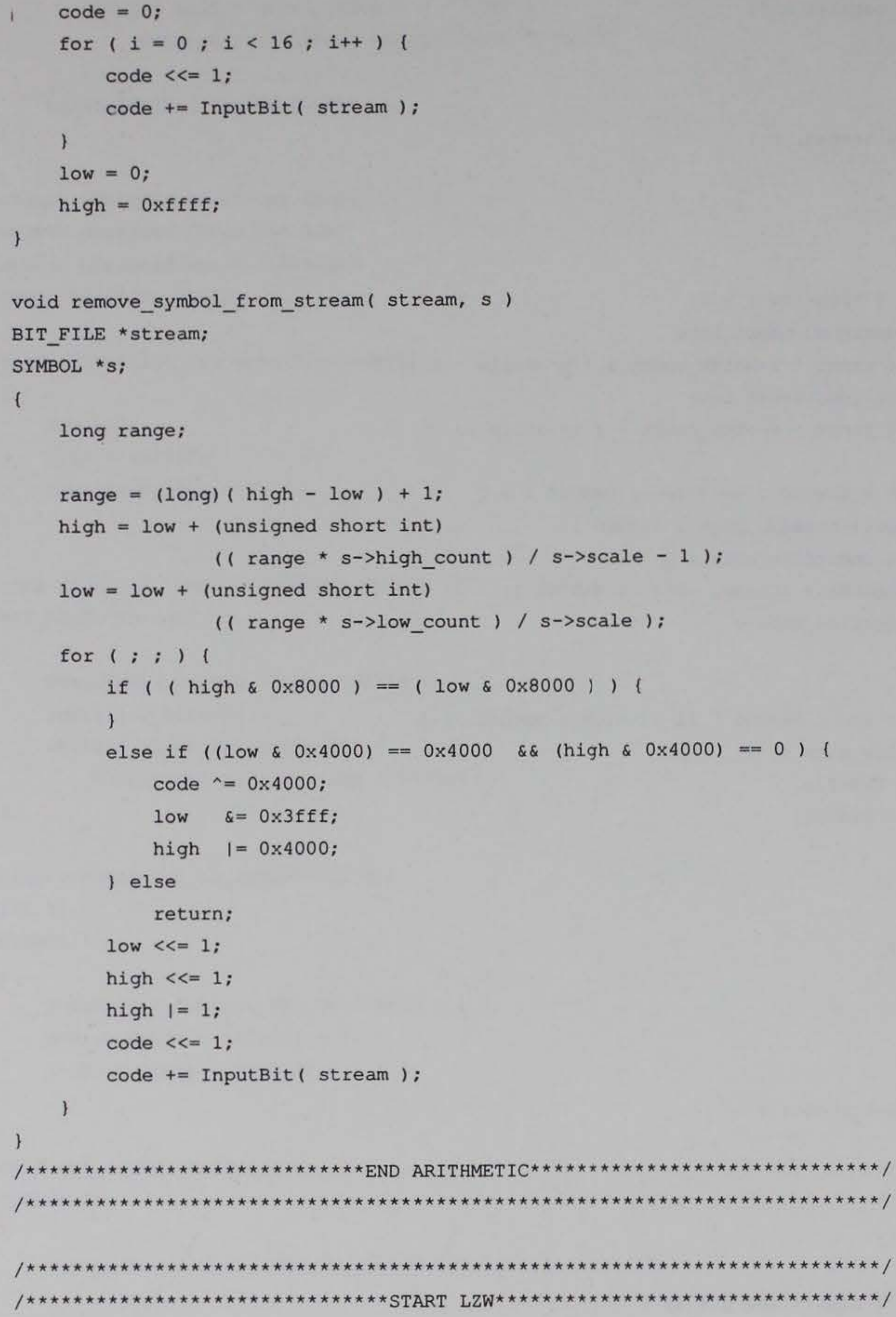

\section{\#define BITS}

\#define MAX_CODE

\#define TABLE_SIZE

\#define EIRST_CODE

\#define UNUSED

\#ifdef _ STDC

void CompressEile (FILE *input, BIT_EILE *output);

void ExpandFile( BIT_EILE *input, FILE *output);

unsigned int find_child_node( int parent_code, int child_character );

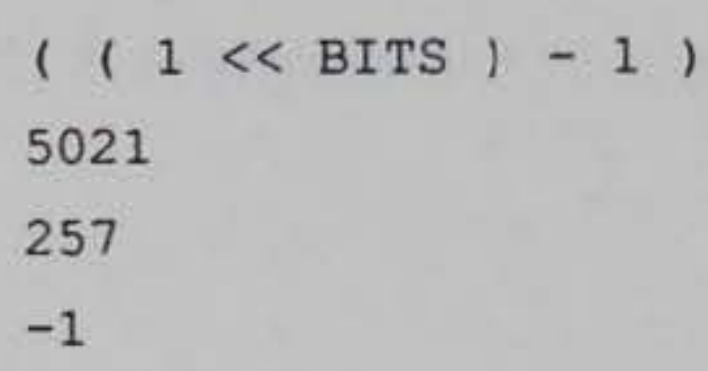




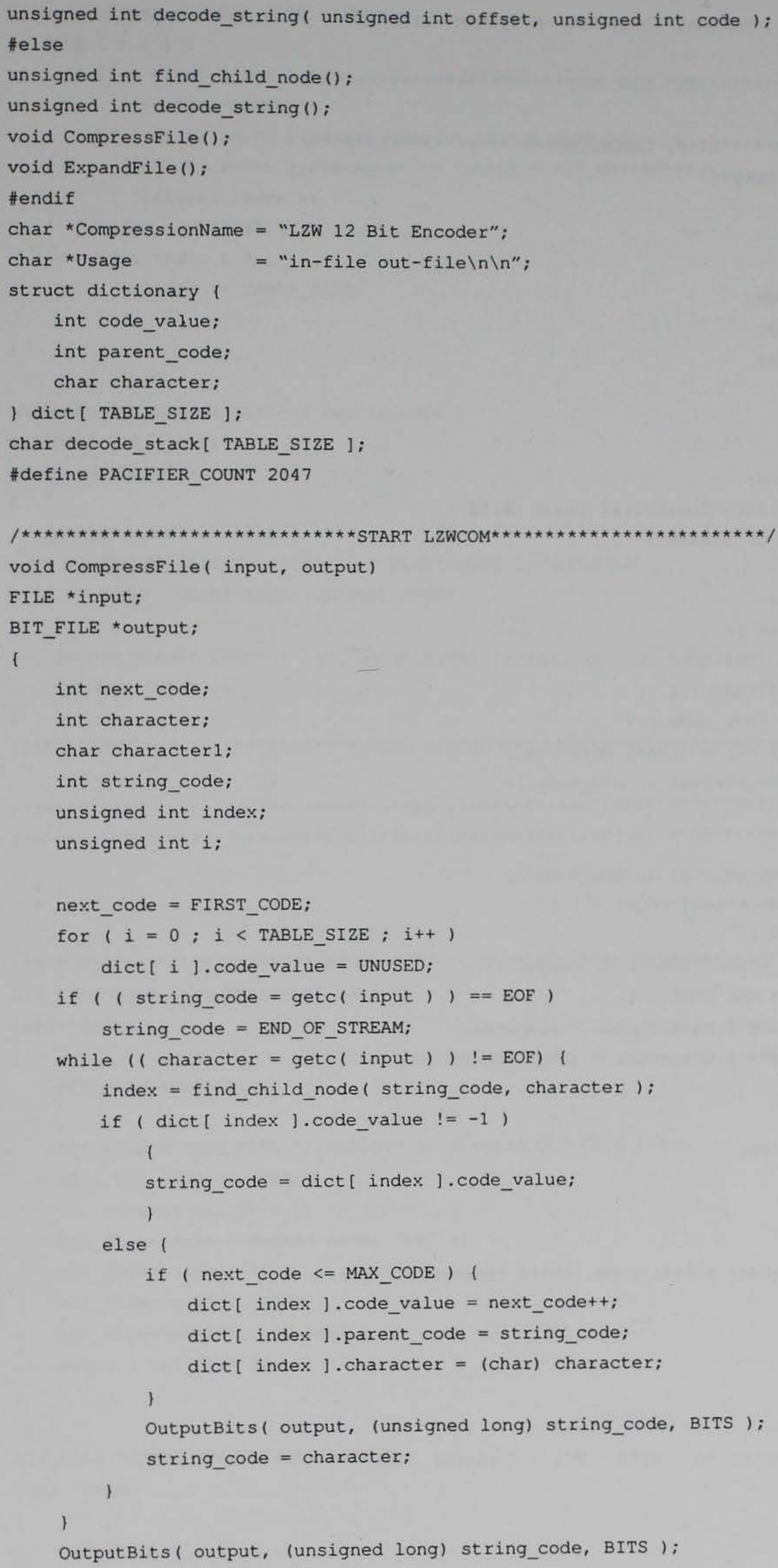




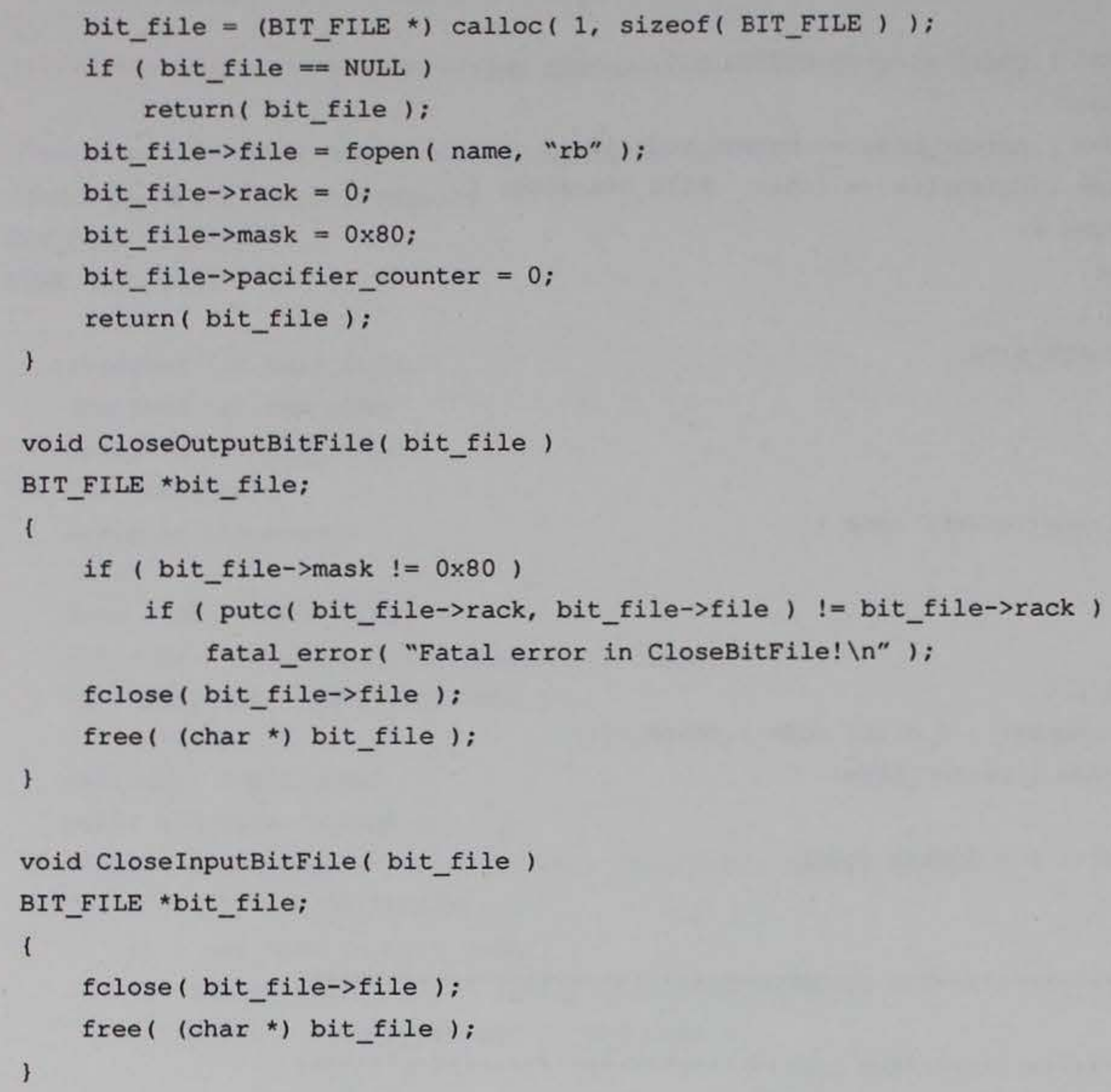




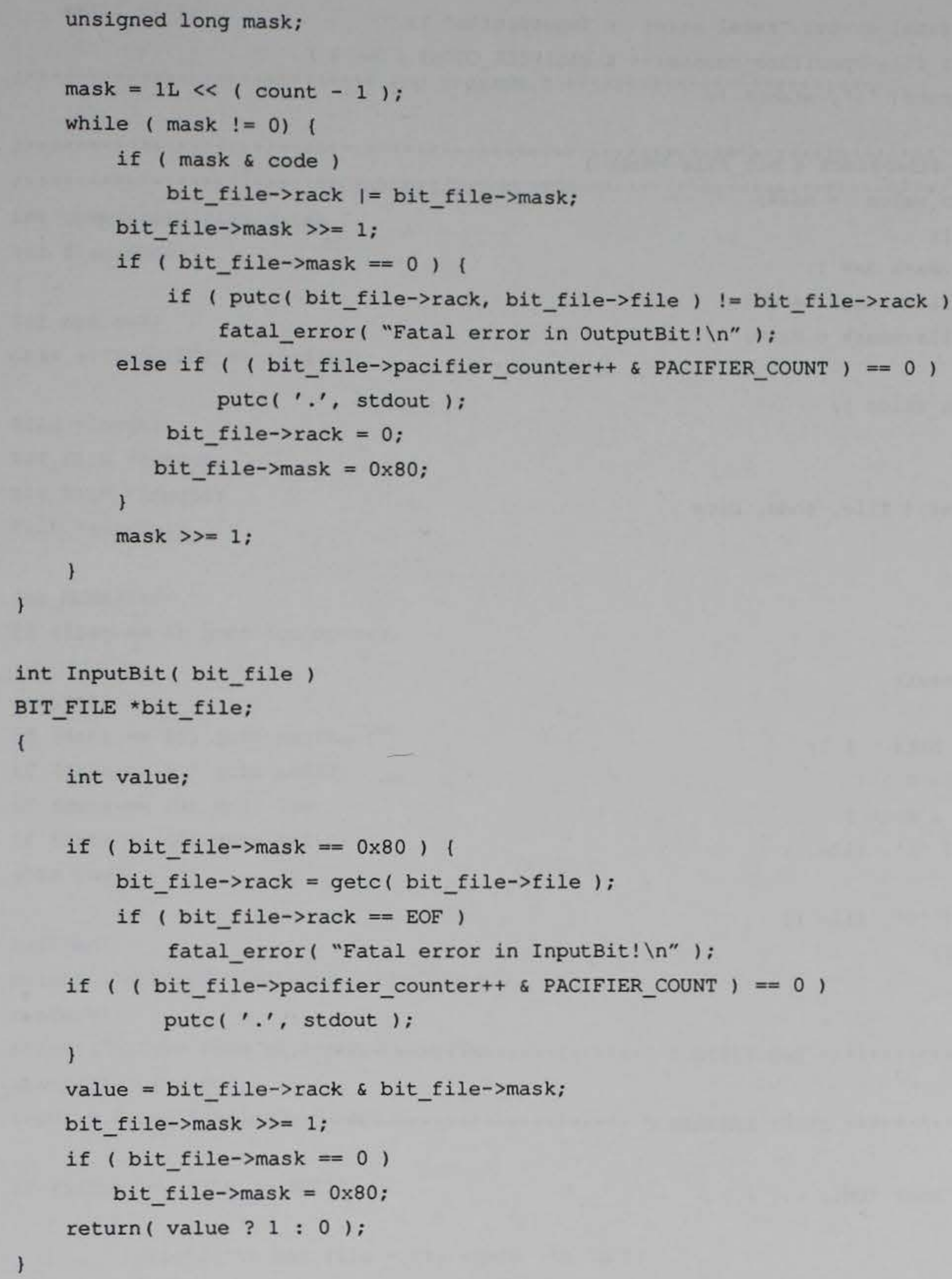




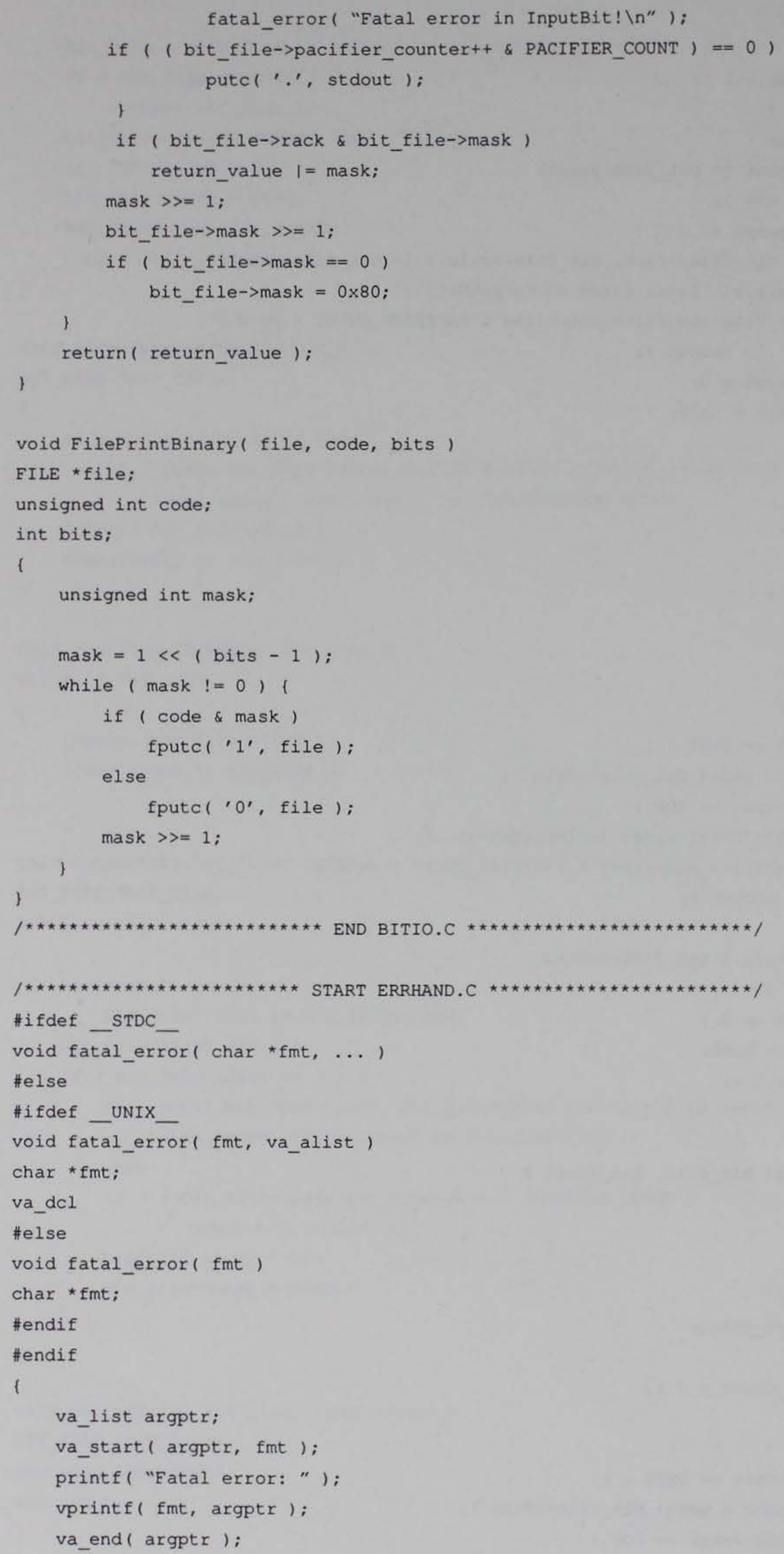




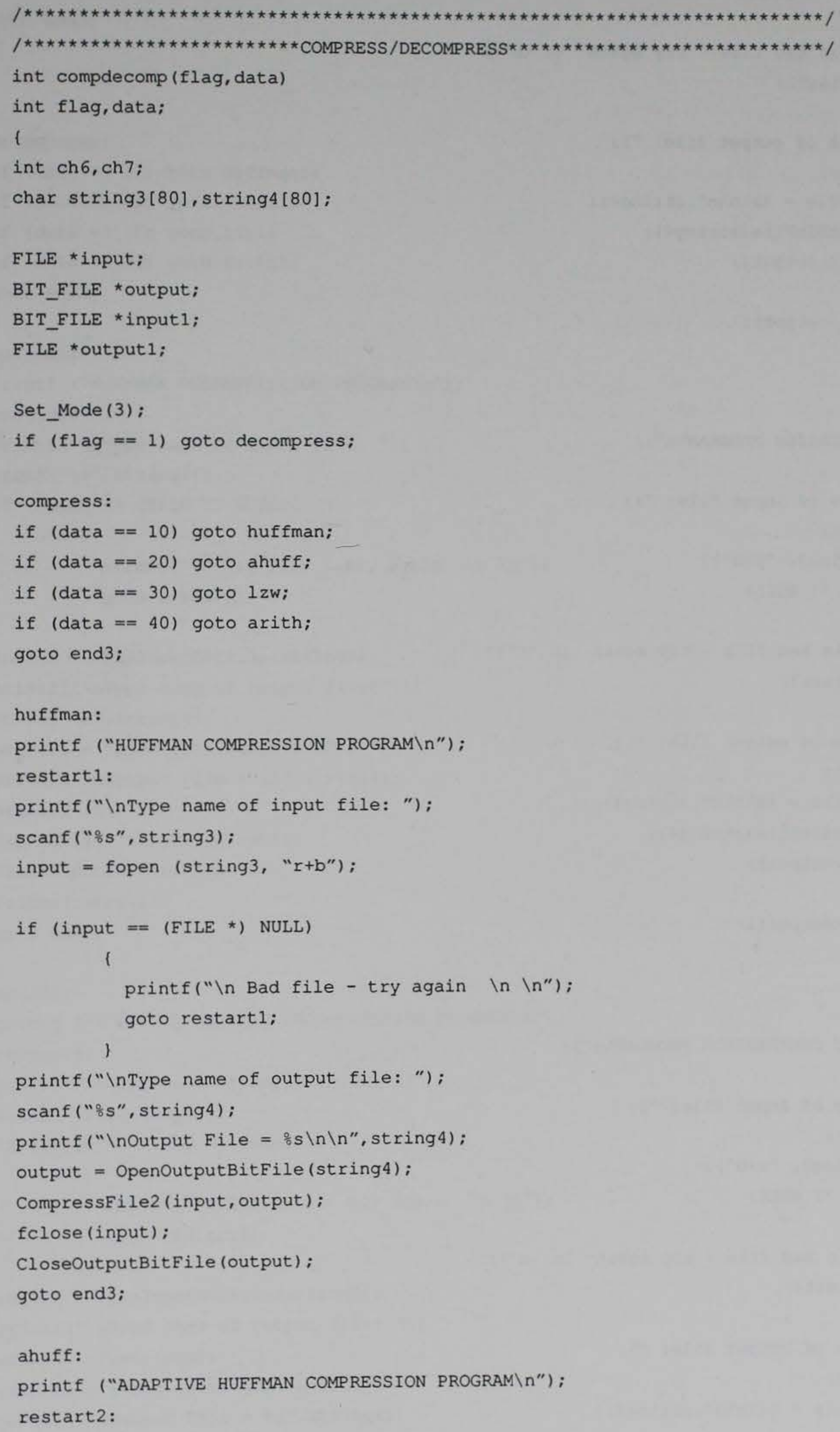




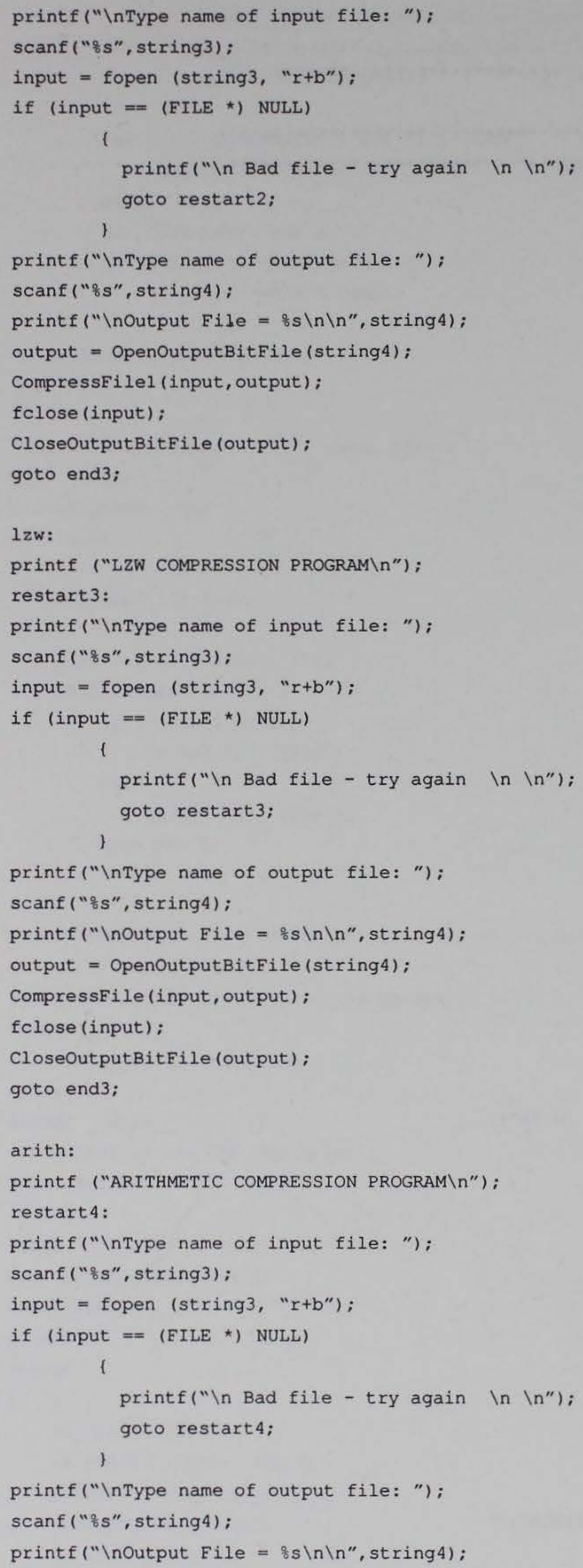


output = OpenOutputBitFile (string 4$)$;

CompressFile3 (input, output);

fclose (input) :

CloseOutputBitFile (output);

goto end3;

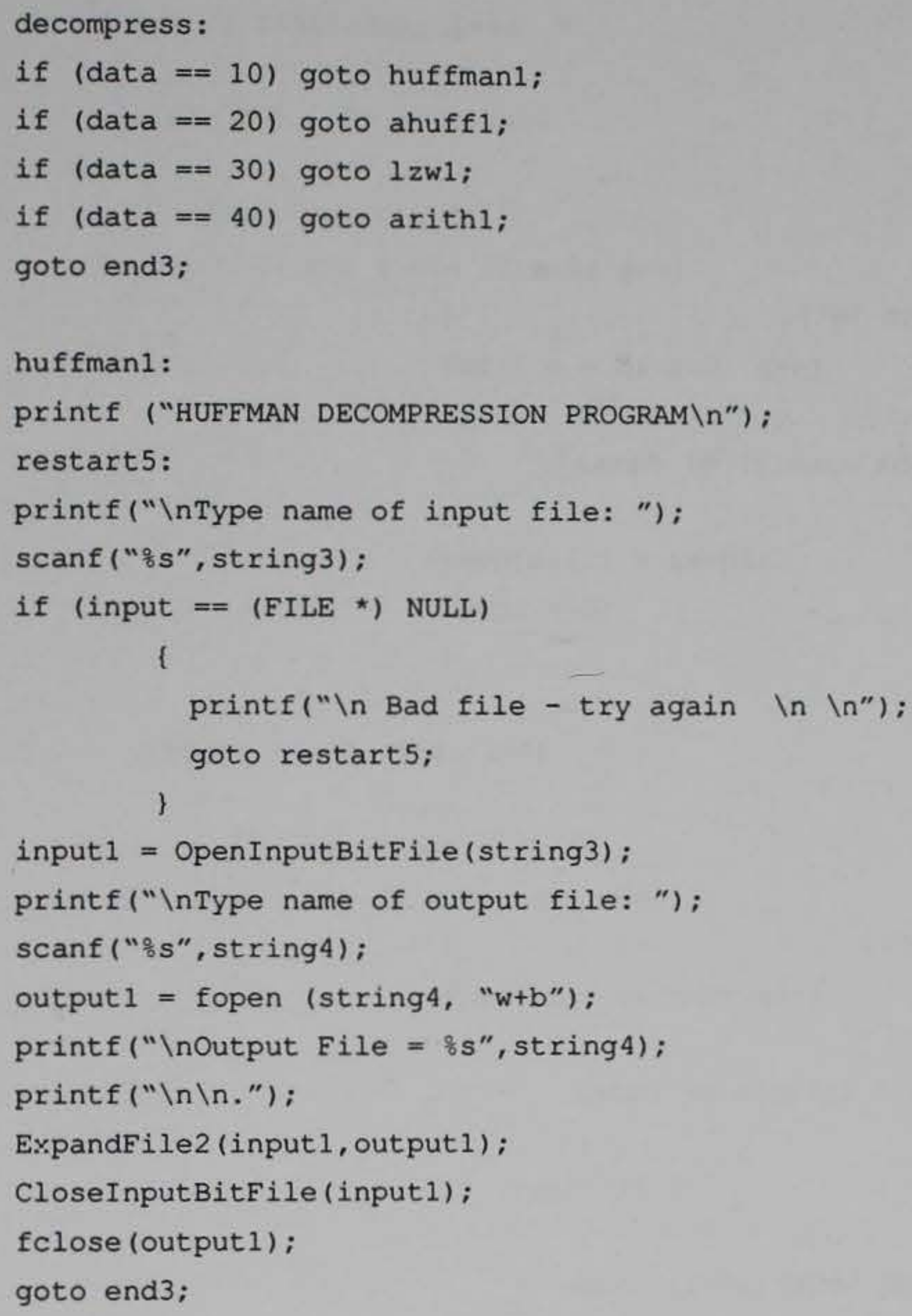




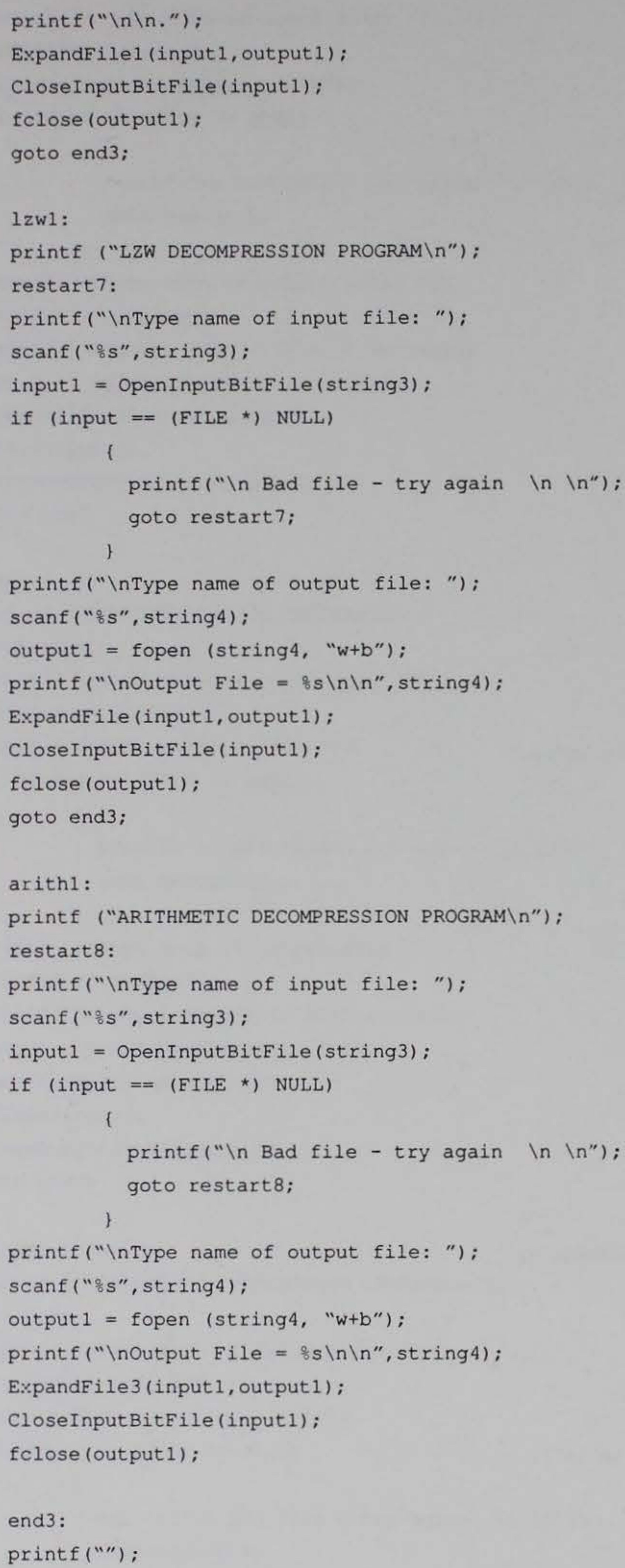




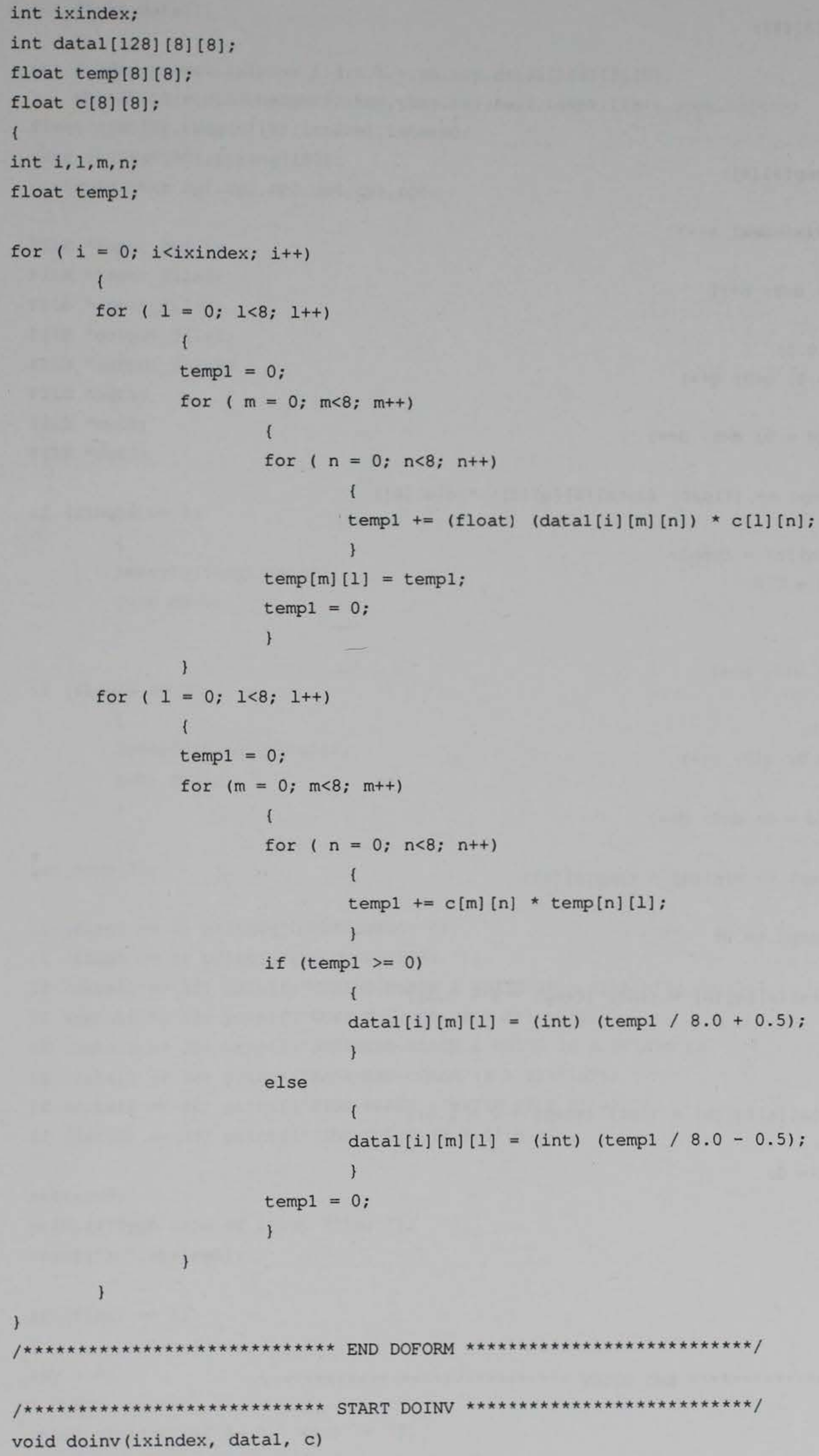

void doinv(ixindex, datal, c)

int ixindex; 


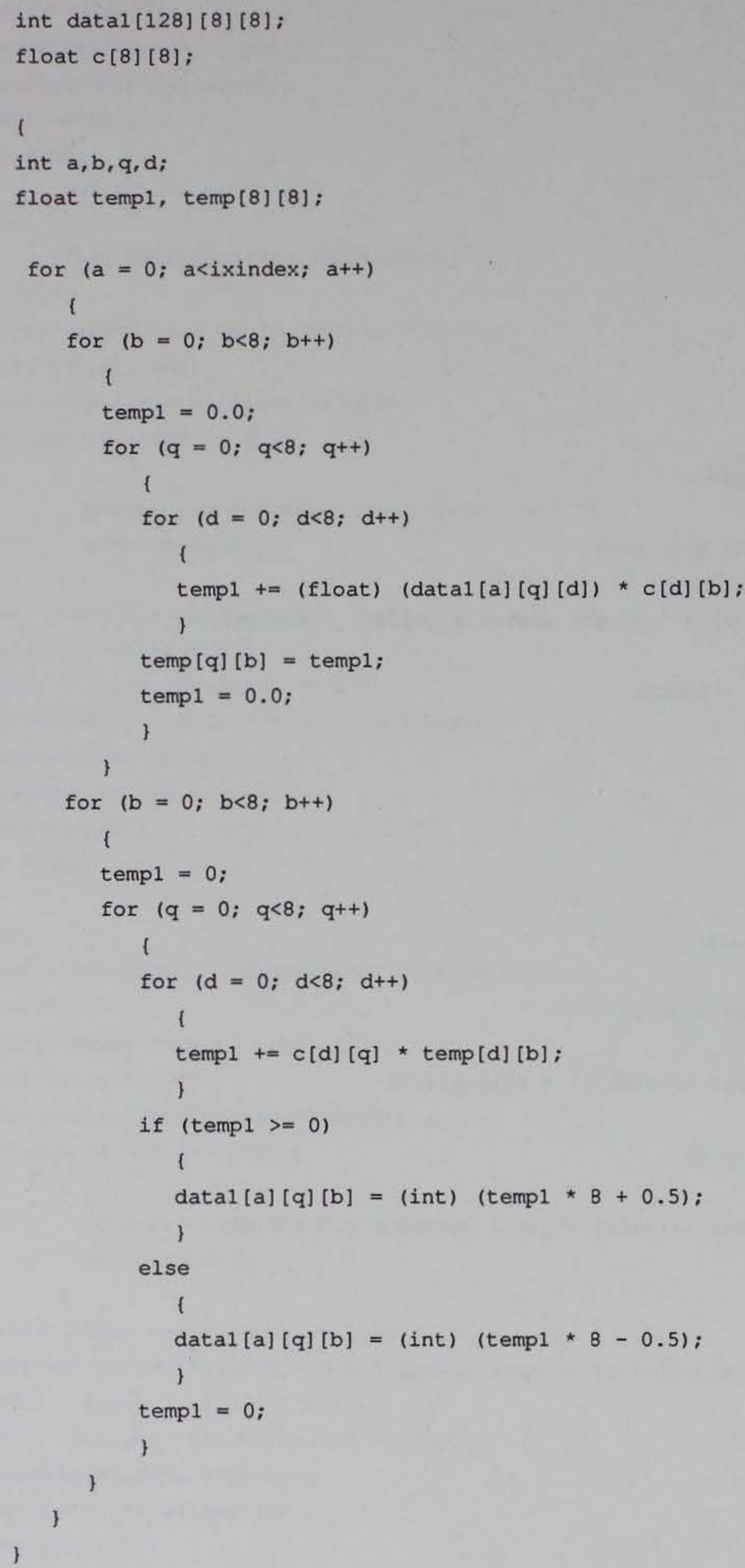




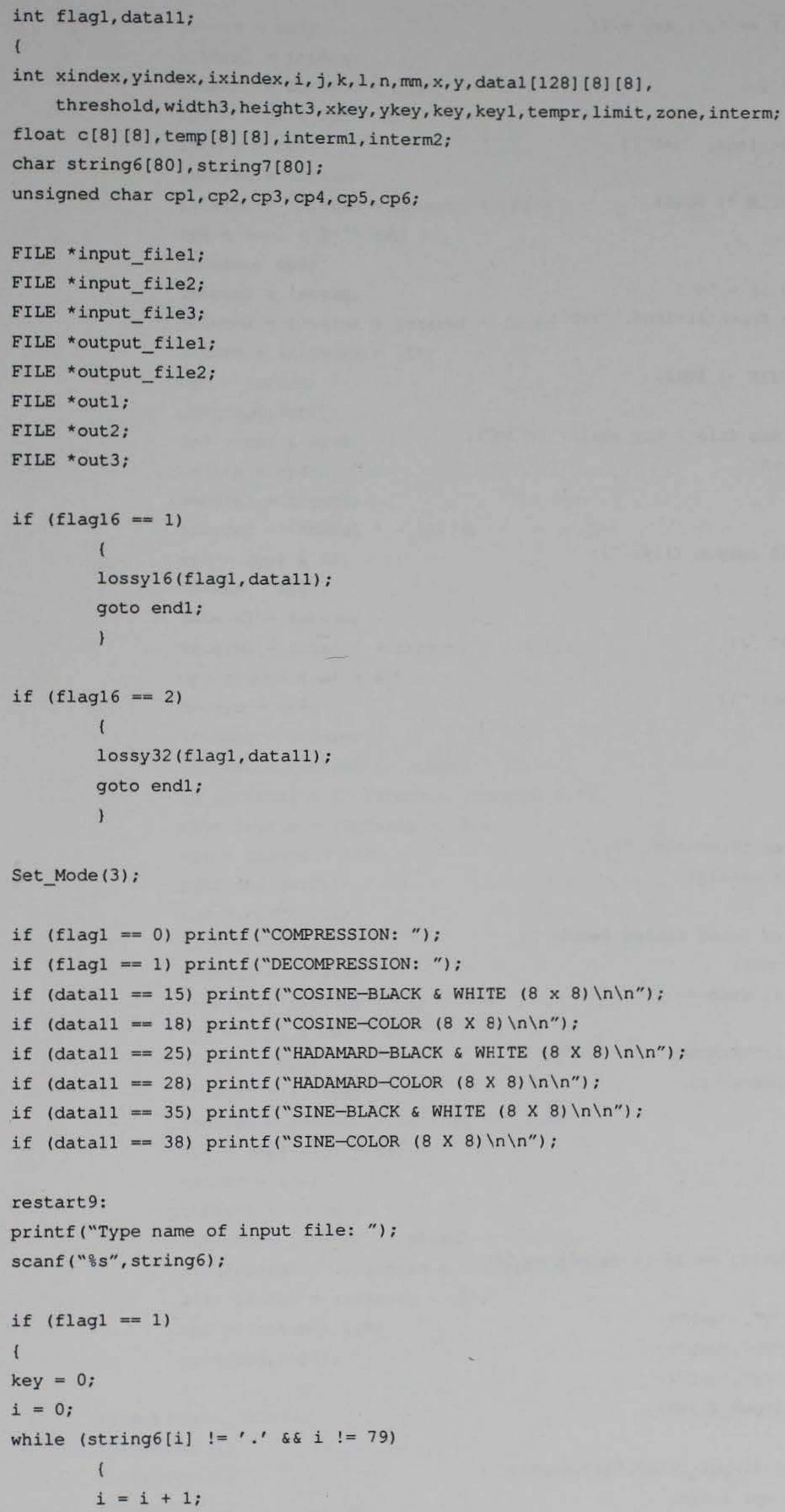




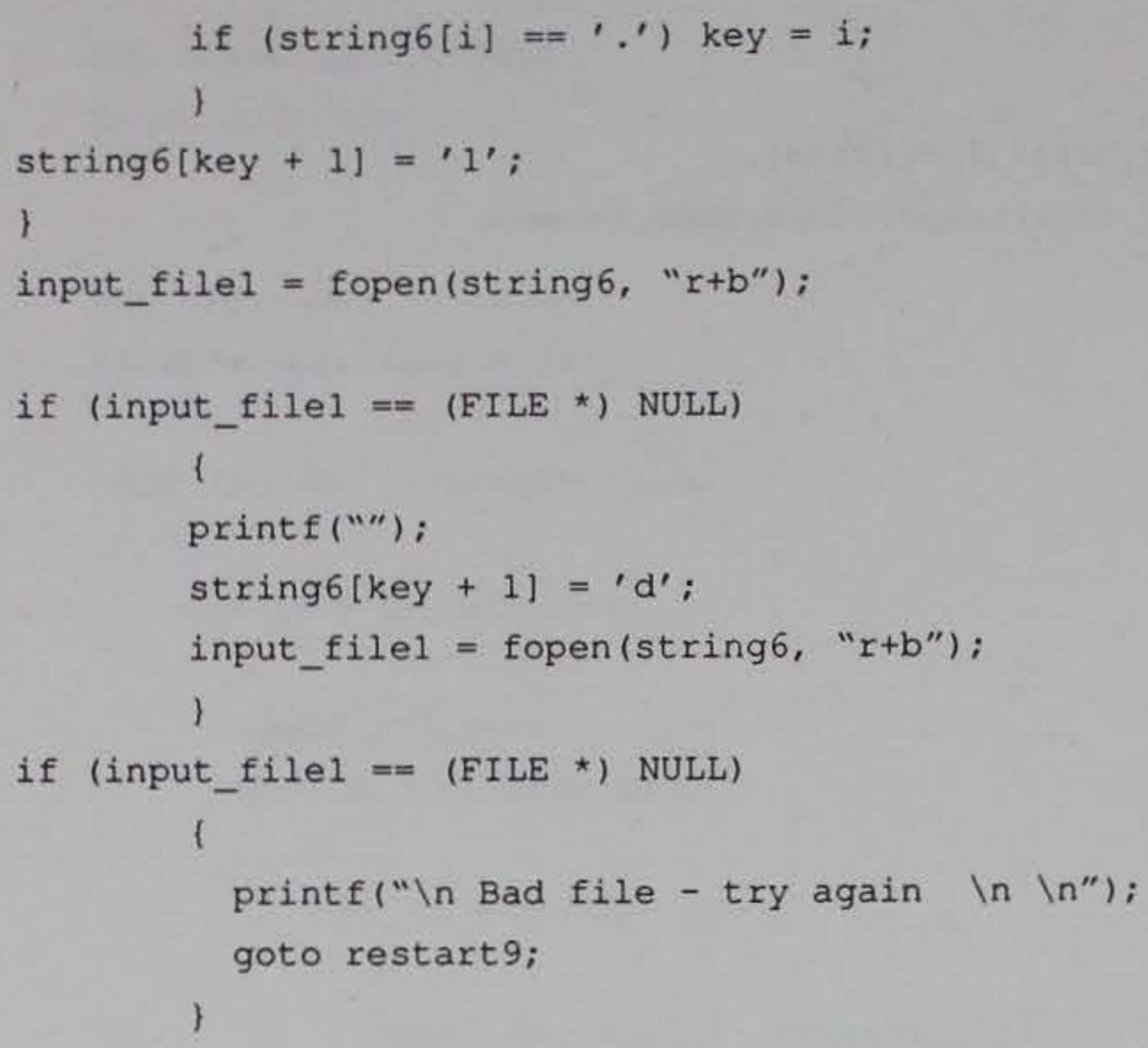




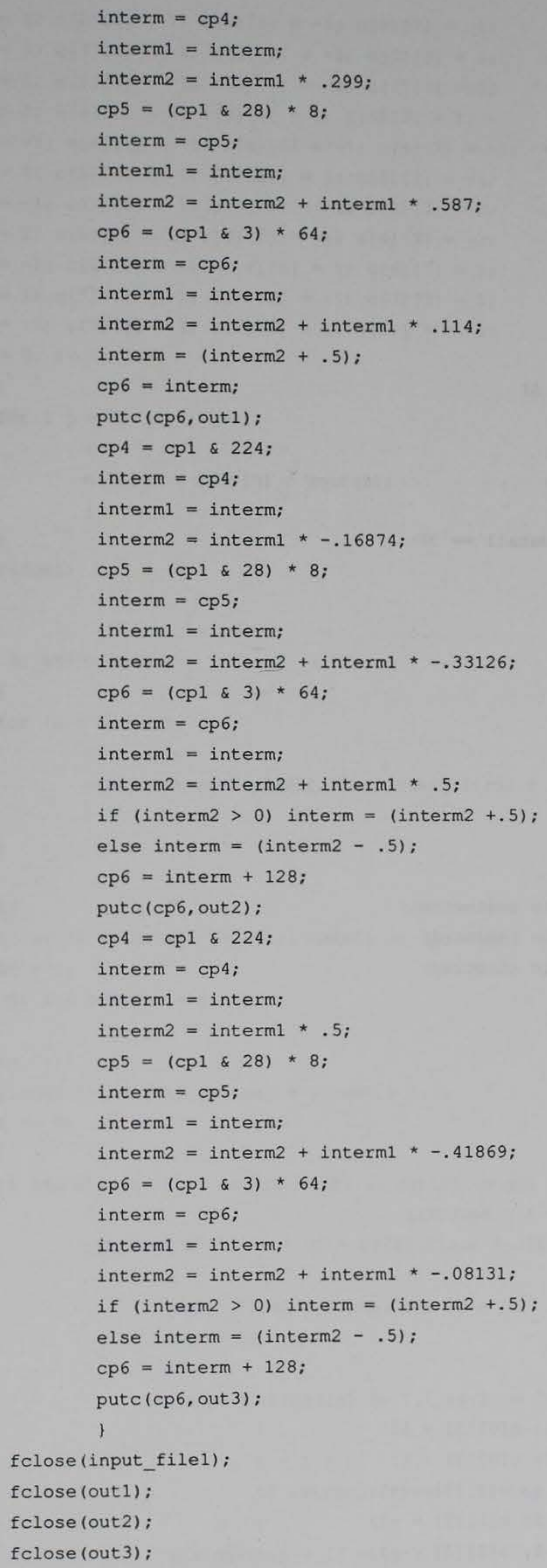




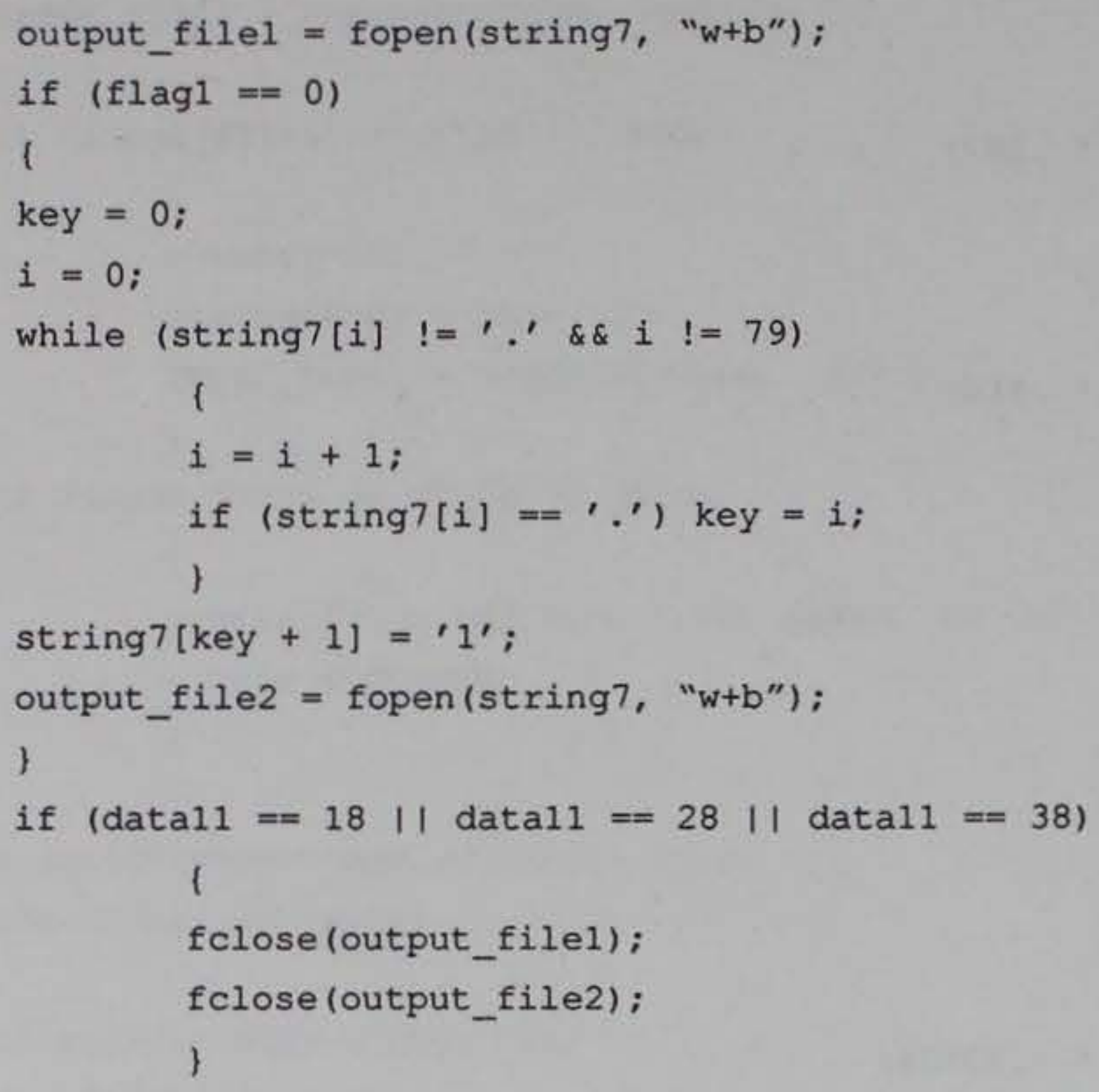

cosinetran:

for $(\mathrm{k}=0 ; \mathrm{k}<=7 ; \mathrm{k}++)$

1

for $(\mathrm{n}=0 ; \mathrm{n}<=7 ; \mathrm{n++})$

1

if $(k==0) c[k][n]=1 / \operatorname{sqrt}(8)$;

else $c[k][n]=\operatorname{sqrt}(.25) * \cos (3.14159 *(2 * n+1) \star k / 16)$;

\}

\}

goto continuel;

hadamard:

$c[0][0]=1 ; c[0][1]=1 ; c[0][2]=1 ; c[0][3]=1 ;$

$c[0][4]=1 ; c[0][5]=1 ; c[0][6]=1 ; c[0][7]=1 ;$

$c[1][0]=1 ; c[1][1]=-1 ; c[1][2]=1 ; c[1][3]=-1 ;$

$c[1][4]=1 ; c[1][5]=-1 ; c[1][6]=1 ; c[1][7]=-1 ;$

$c[2][0]=1 ; c[2][1]=1 ; c[2][2]=-1 ; c[2][3]=-1 ;$ 


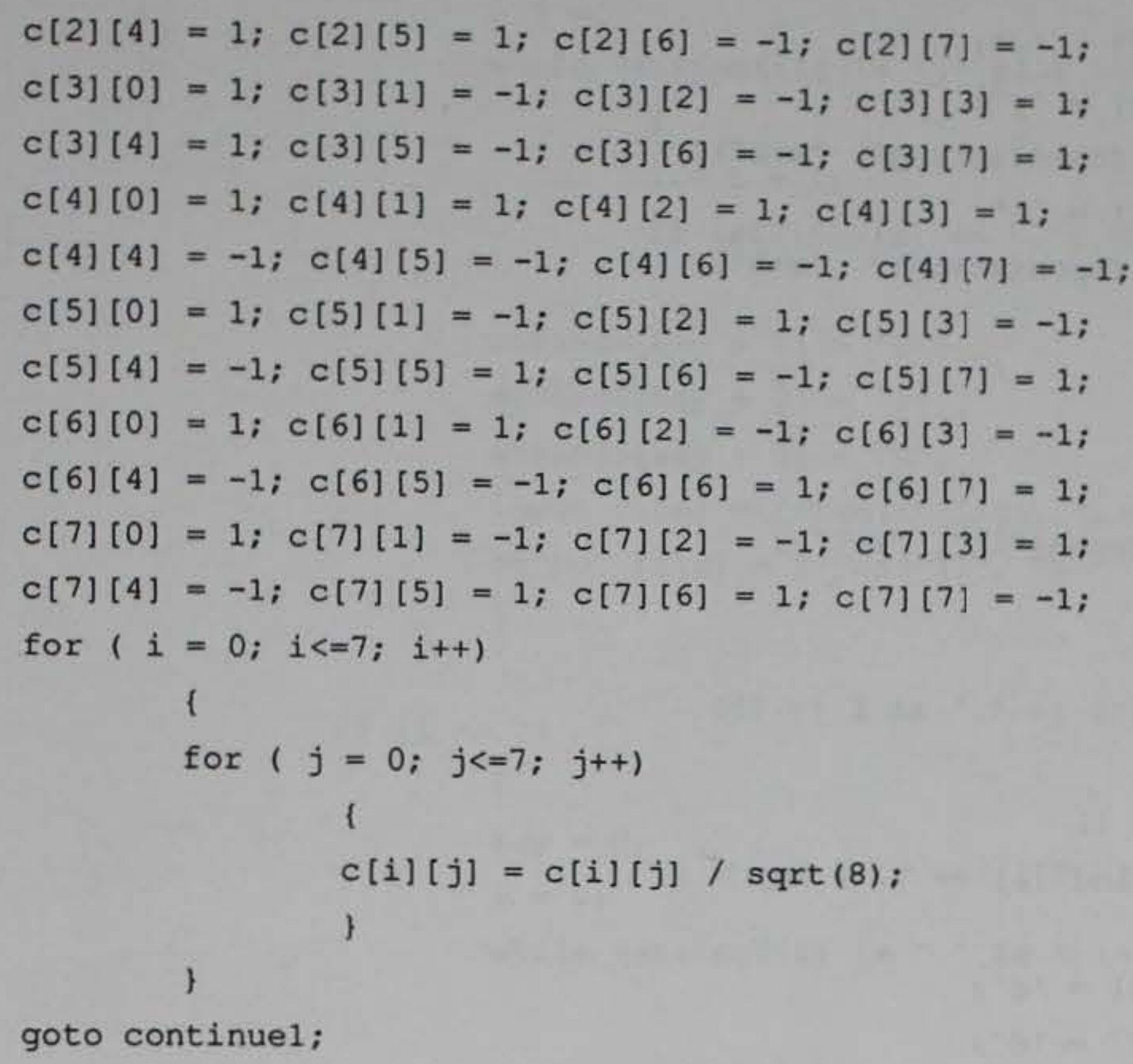




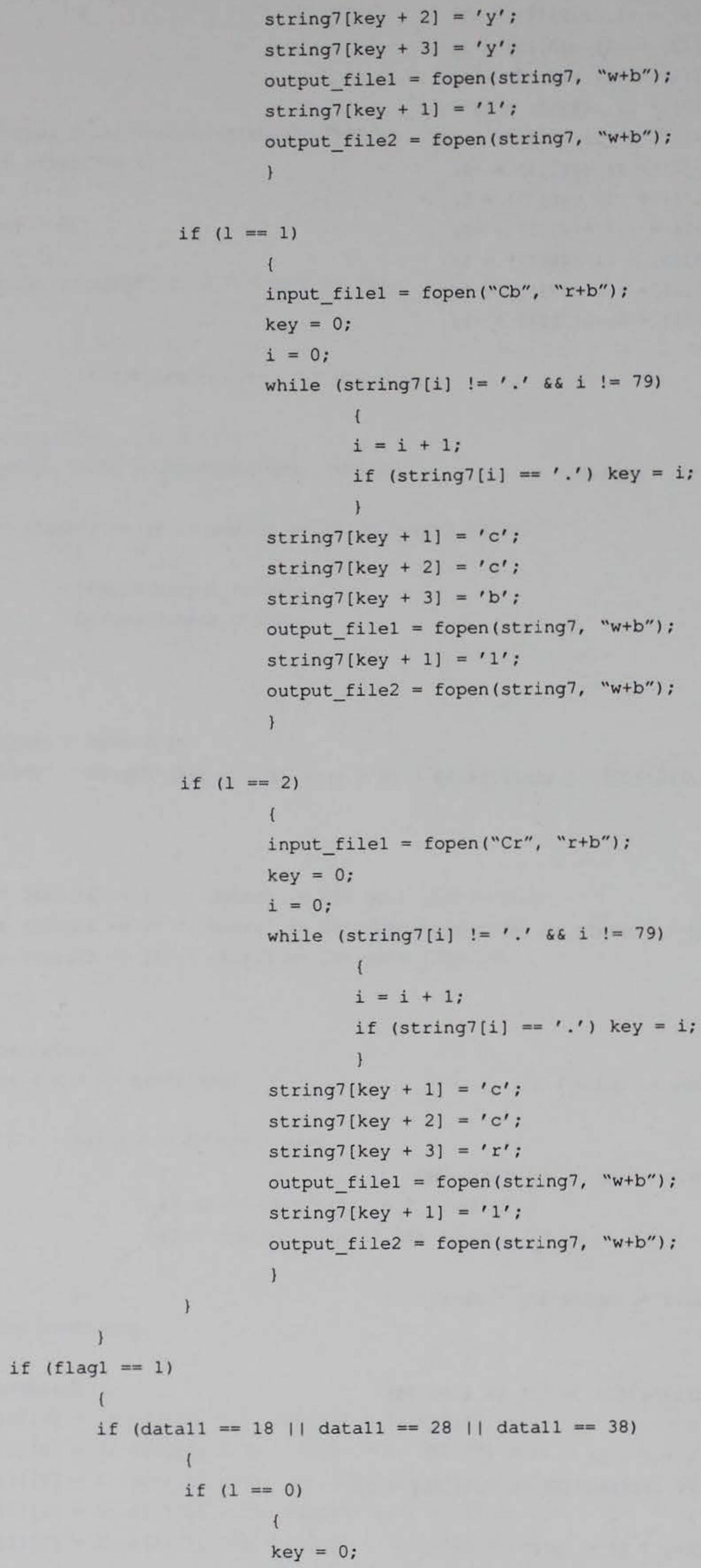




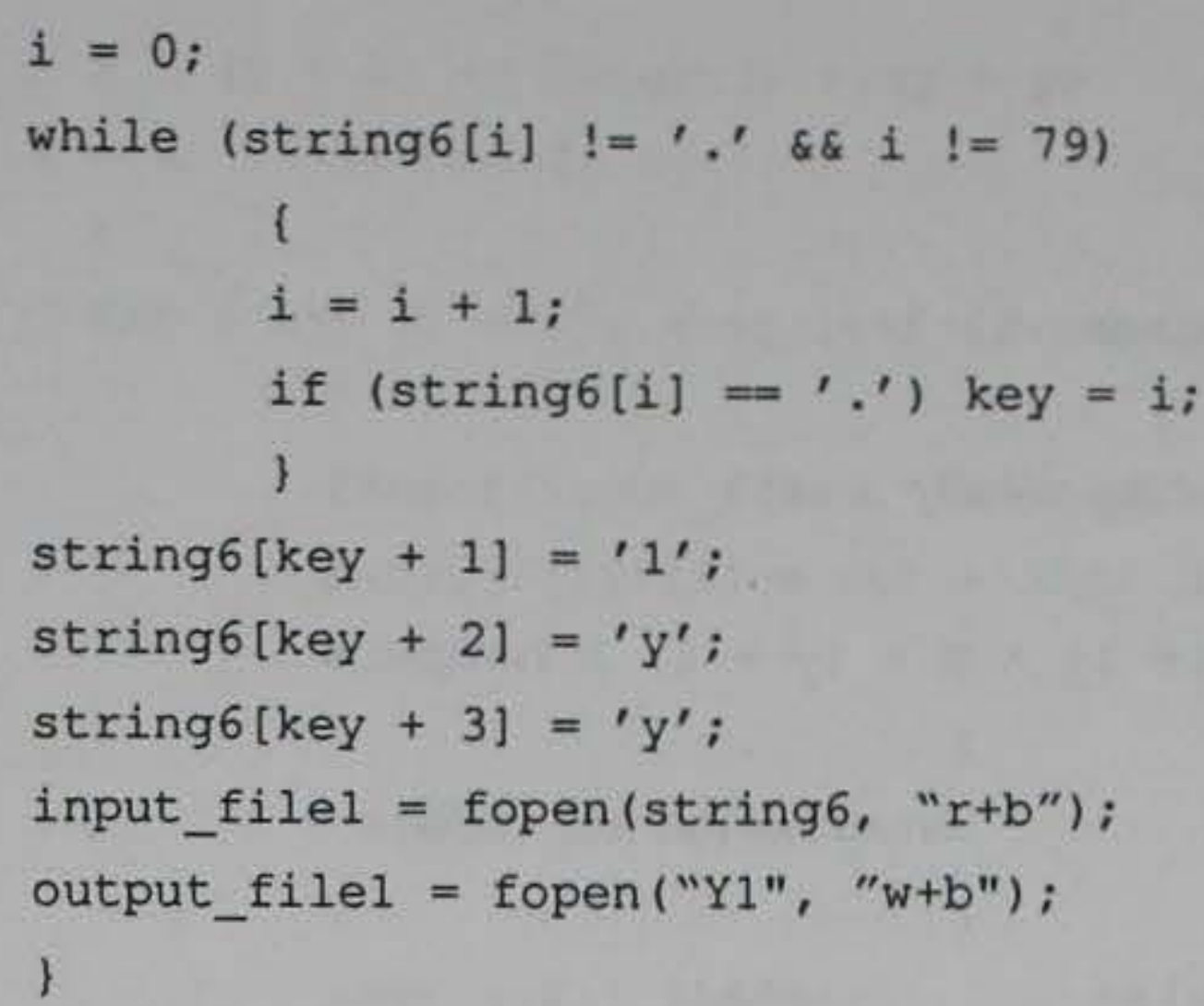

1

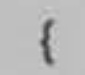




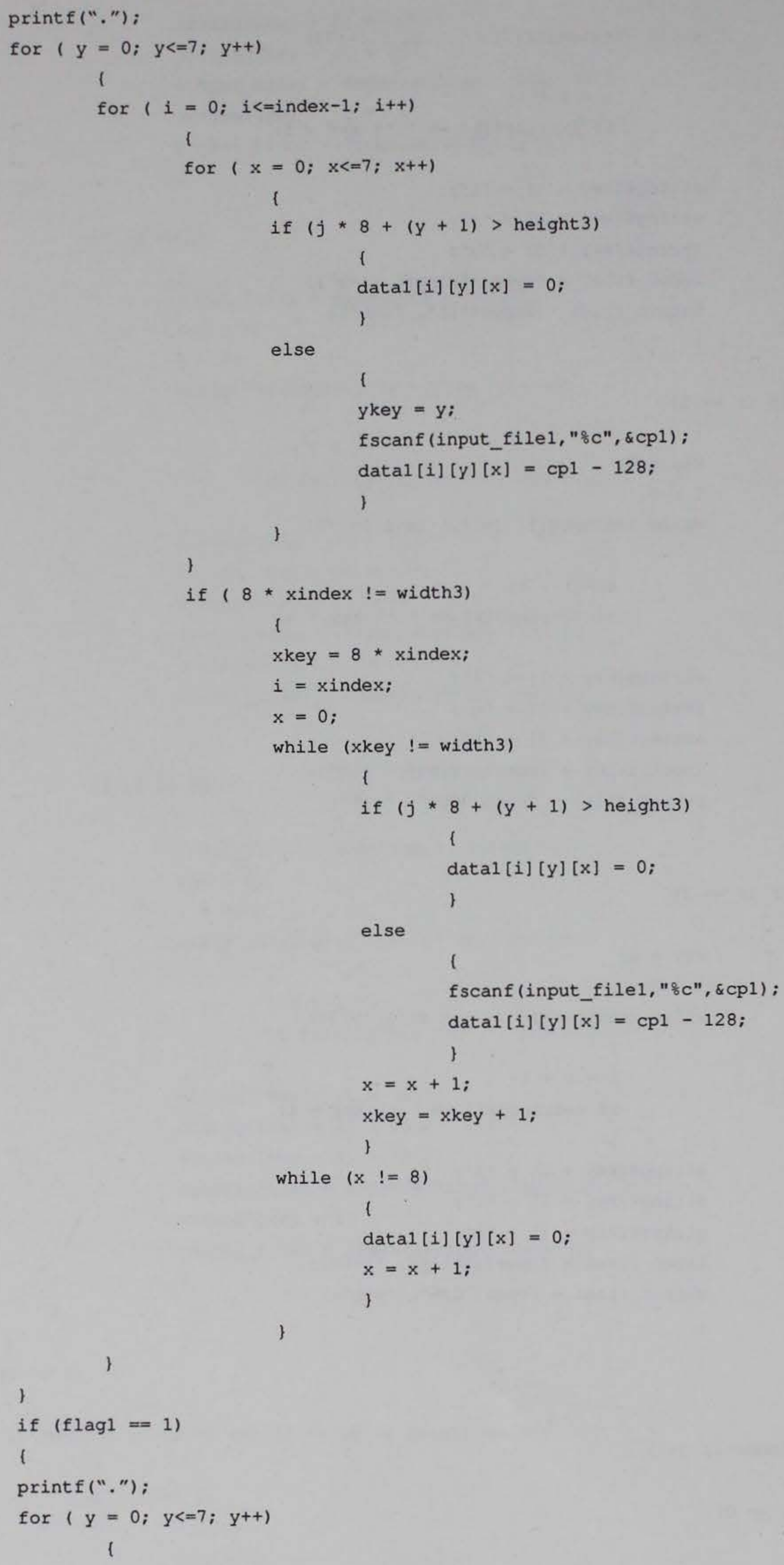




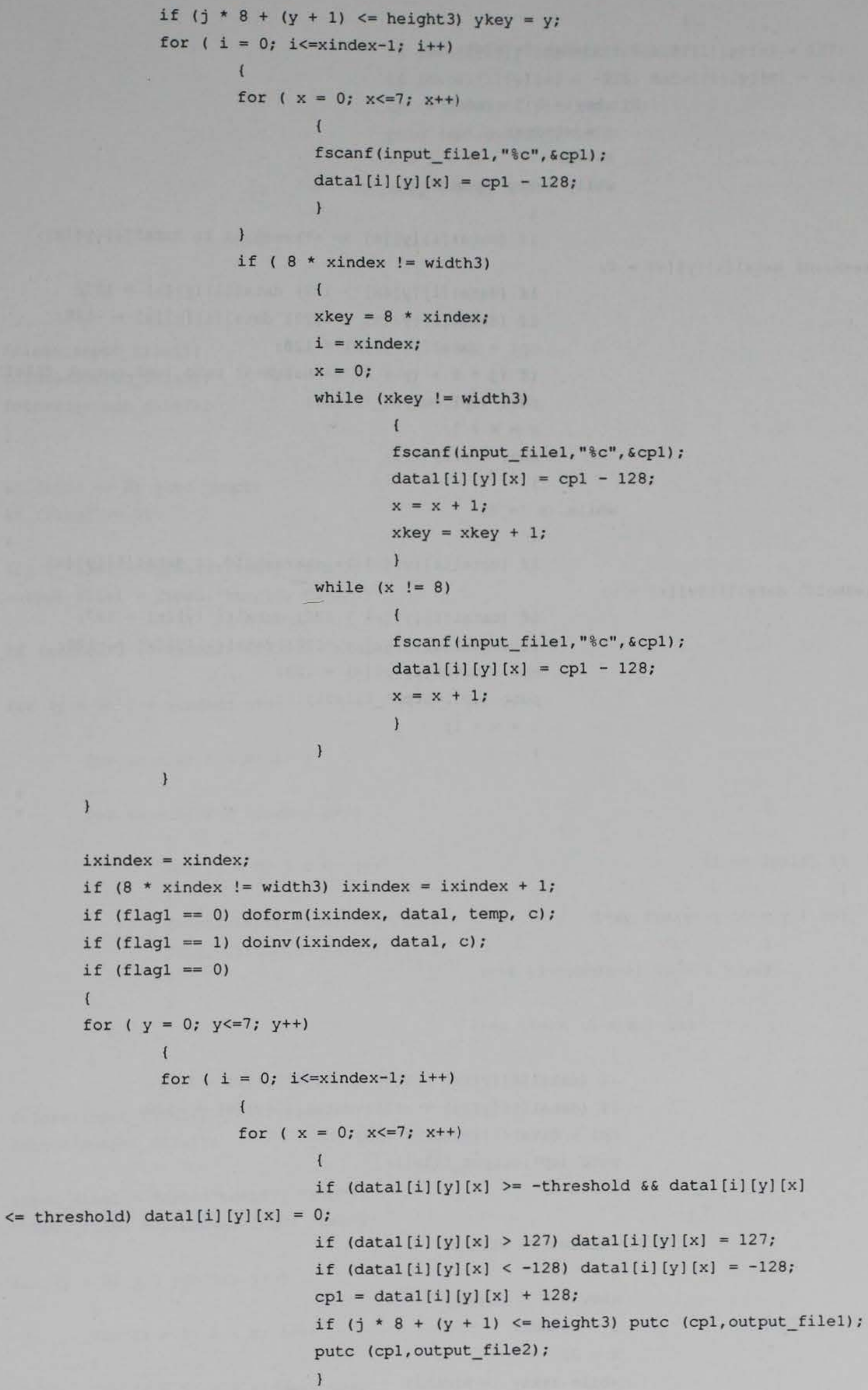


)

if $(8 *$ xindex $!=$ width 3$)$

f

xkey $=8$ * xindex;

$i=x$ index;

$\mathrm{x}=0$;

while (xkey $!=$ width 3 )

1

if (datal $[i][y][x]>=-$ threshold \&\& datal $[i][y][x]$

$<=$ threshold) $\operatorname{datal}[i][y][x]=0$;

if (datal[i] $[y][x]>127)$ datal[i][y] $[x]=127$;

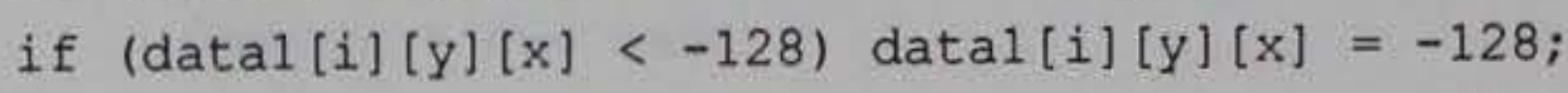

$\mathrm{cp} 1=\operatorname{datal}[\mathrm{i}][\mathrm{y}][\mathrm{x}]+128$;

if $(j * 8+(y+1)<=$ height 3$)$ putc (cpl, output_file 1$)$;

putc (cpl, output_file2);

$\mathrm{x}=\mathrm{x}+1$;

$\mathrm{xkey}=\mathrm{xkey}+1$;

\}

while $(x:=8)$

1

if (datal[i] $[y][x]>=-$ threshold $\& \&$ datal $[i][y][x]$

threshold) $\operatorname{datal}[i][y][x]=0$;

if (datal[i][y][x]>127) datal[i][y] $[\mathrm{x}]=127$;

if (datal $[i][y][x]<-128)$ datal $[i][y][x]=-128$;

$\mathrm{cp} 1=\operatorname{data}[1][y][\mathrm{x}]+128$;

putc (cpl, output_file2);

$x=x+1$

\}

\}

)

1

if $(\mathrm{flag} 1==1)$

1

for $\left(\mathrm{y}=0 ; \mathrm{y}\right.$ <=ykey; $\left.\mathrm{y}^{++}\right)$

1

for $(i=0$; $i<=x i n d e x-1 ; i++)$

i

for $(x=0 ; x<=7 ; x++)$

1

if (datal[i][y] $[\mathrm{x}]>127)$ datal[i][y] $[\mathrm{x}]=127$;

if (datal[i] $[y][x]<-128)$ datal[i][y] $[x]=-128$;

$\mathrm{cp} 1=\operatorname{data}[i][y][x]+128$;

putc (cpl, output_filel);

।

\}

if $(8 *$ xindex $!=$ width 3$)$

\{

xkey $=8 * x$ index;

$i=x$ index;

$x=0$;

while (xkey $!=$ width 3 ) 


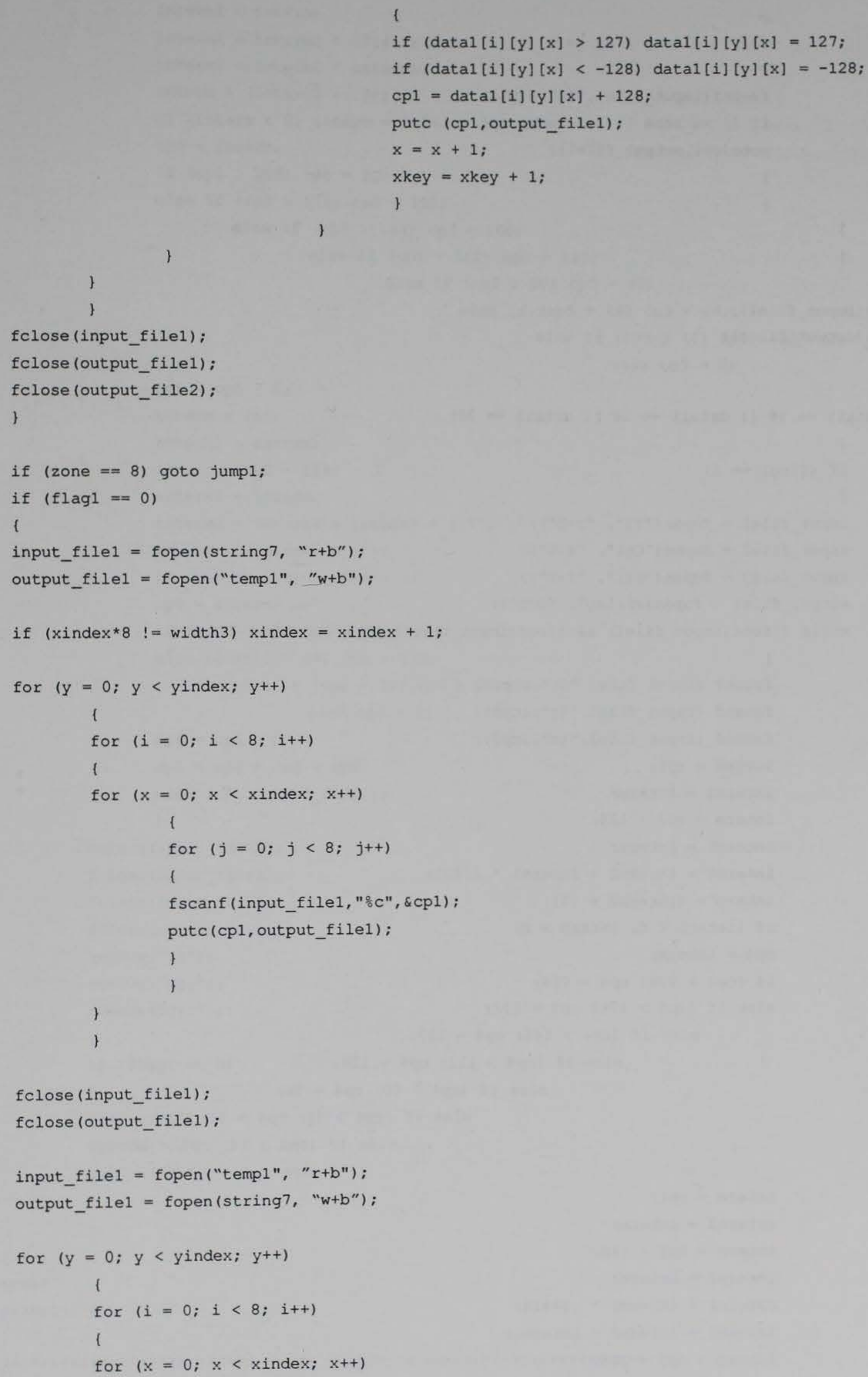


1

for $(j=0 ; j<8 ; j++)$

1

fscanf(input_file1, "\%c", \&cpl) ;

if ( $\mathrm{i}>=$ zone || $\mathrm{j}>=$ zone) $\mathrm{cp} 1=128$;

putc (cpl, output_file1);

\}

)

)

)

fclose (input_filel);

fclose (output_filel);

)

jump1:

if $($ datal $1==18||$ datall $==28||$ datall $==38$ )

1

if $(f \operatorname{lag} 1==1)$

f

input_file1 = fopen ("Y1", " $r+b ")$;

input_file2 = fopen ("Cbl", " $r+b ")$;

input_file3 = fopen ("Crl", " $r+b ")$;

output_filel = fopen (string7, " $w+b ")$;

while (!feof(input_file1) \&\& !feof(input_file2) \&\& !feof(input_file3))

1

fscanf (input_filel," \%c", \&cp1);

fscanf (input_file2," $\% "$ " \&cp 2 );

fscanf (input_file $3, " \% c ", \& c p 3)$;

interm $=\mathrm{cp} 1$;

interm2 = interm;

interm $=\mathrm{cp} 3-128$;

interm1 = interm;

interm2 $=$ interm2 + interm1 * 1.402 ;

interm $=($ interm $2+.5)$;

if $($ interm $<0$ ) interm $=0$;

$\mathrm{cp} 4=$ interm;

if $(\mathrm{cp} 4>208) \quad \mathrm{cp} 4=224$;

else if $(\mathrm{cp} 4>176) \mathrm{cp} 4=192$;

else if $(\mathrm{cp} 4>144) \mathrm{cp} 4=160$;

else if $(\mathrm{cp} 4>112) \mathrm{cp} 4=128$;

else if $(\mathrm{cp} 4>80) \mathrm{cp} 4=96$;

else if $(\mathrm{cp} 4>48) \mathrm{cp} 4=64$;

else if $(\mathrm{cp} 4>16) \quad \mathrm{cp} 4=32$;

else $\mathrm{cp} 4=0$;

interm $=\mathrm{cpl}$;

interm2 = interm;

interm $=\mathrm{cp} 2-128$;

interm1 = interm;

interm1 $=$ interm 1 * 34414 ;

interm2 = interm2 - interm1;

interm $=\mathrm{cp} 3-128$; 


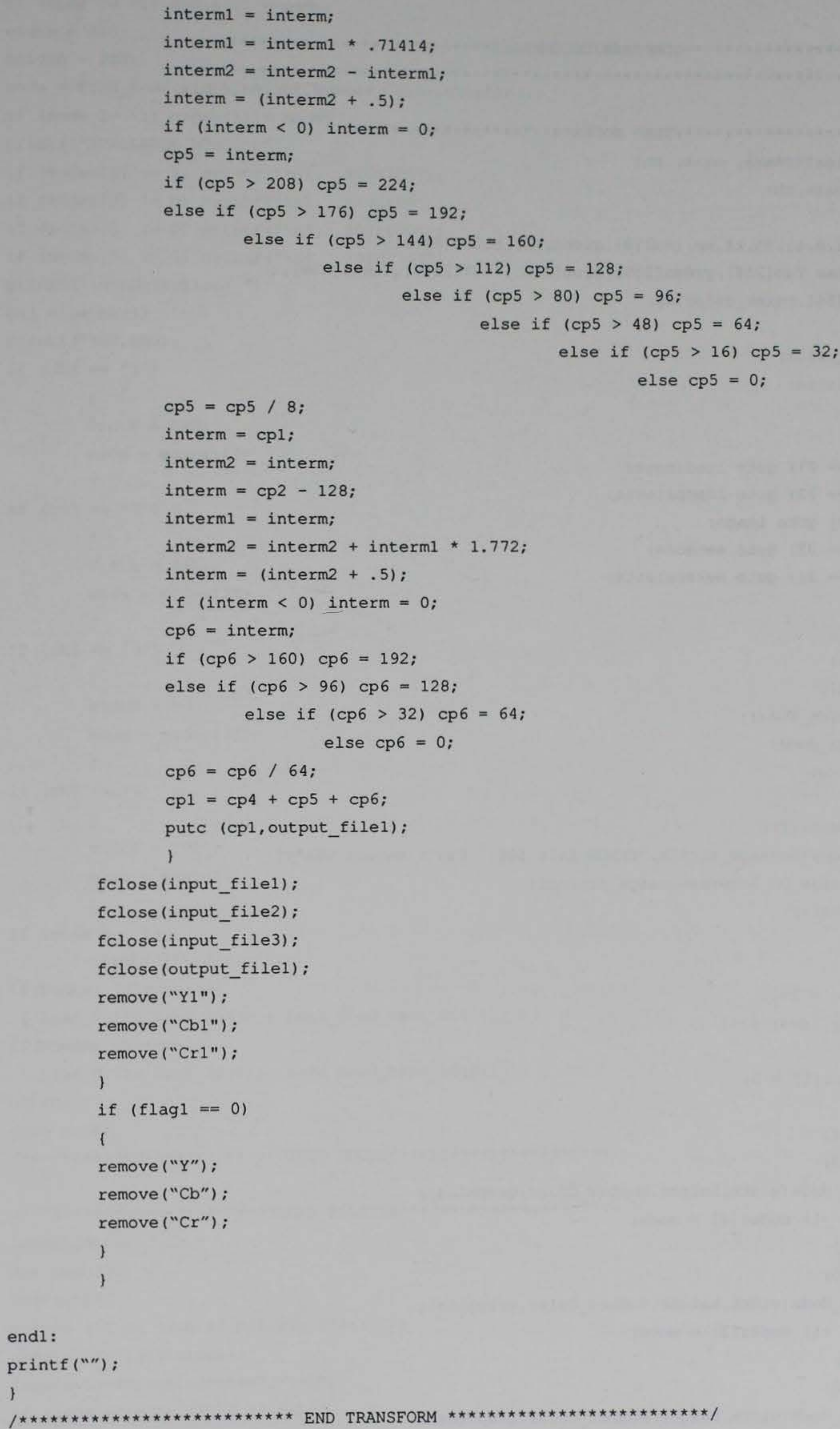




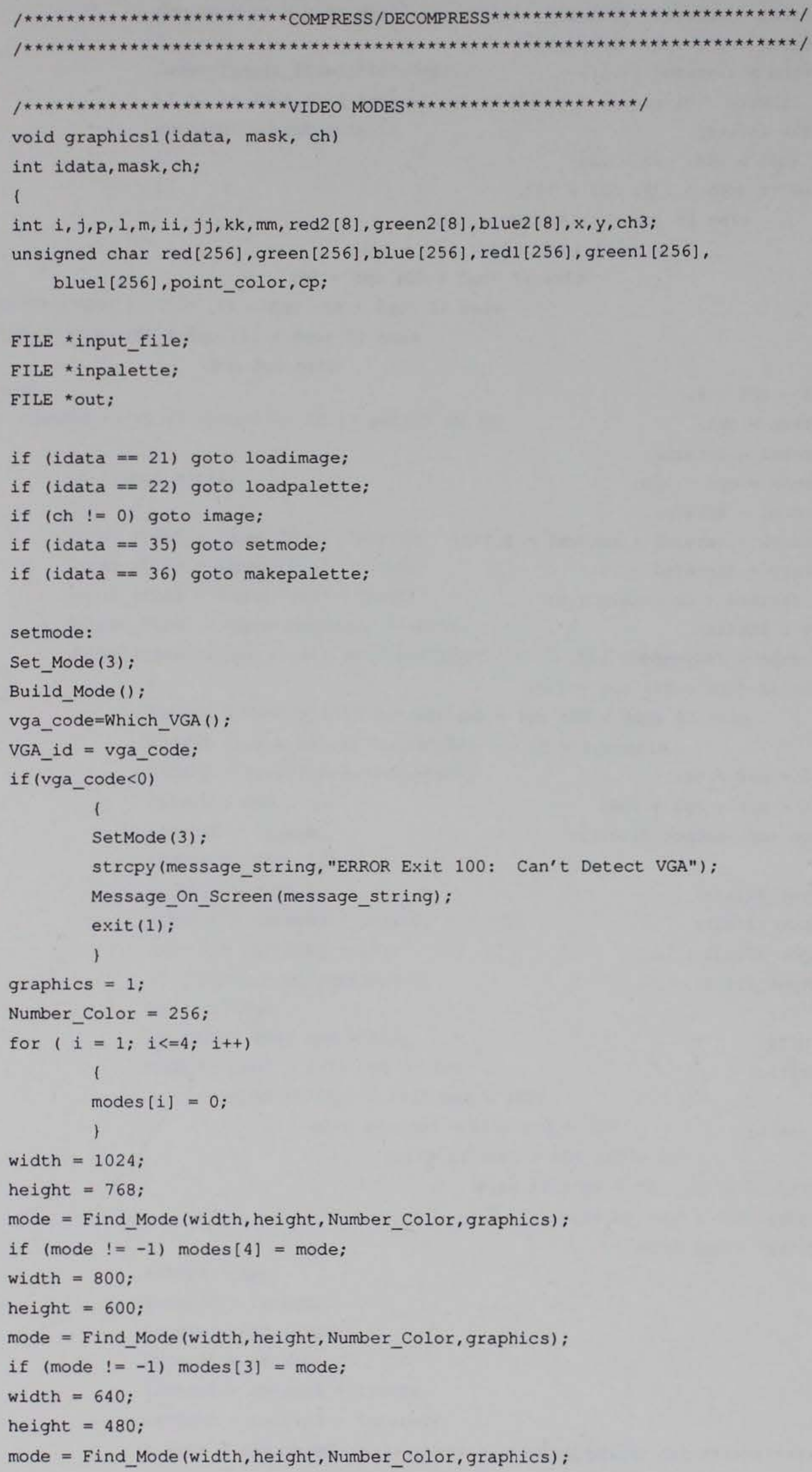




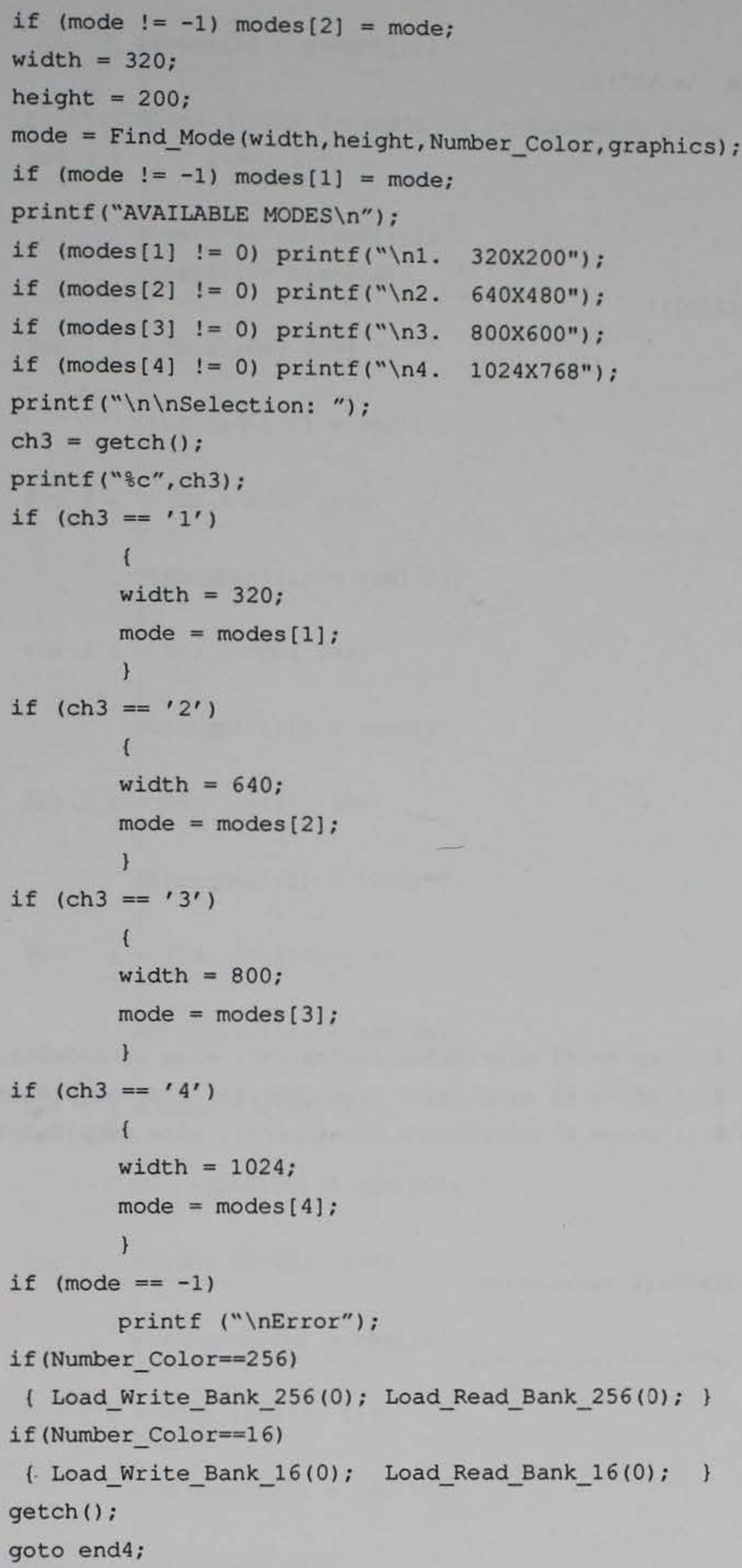




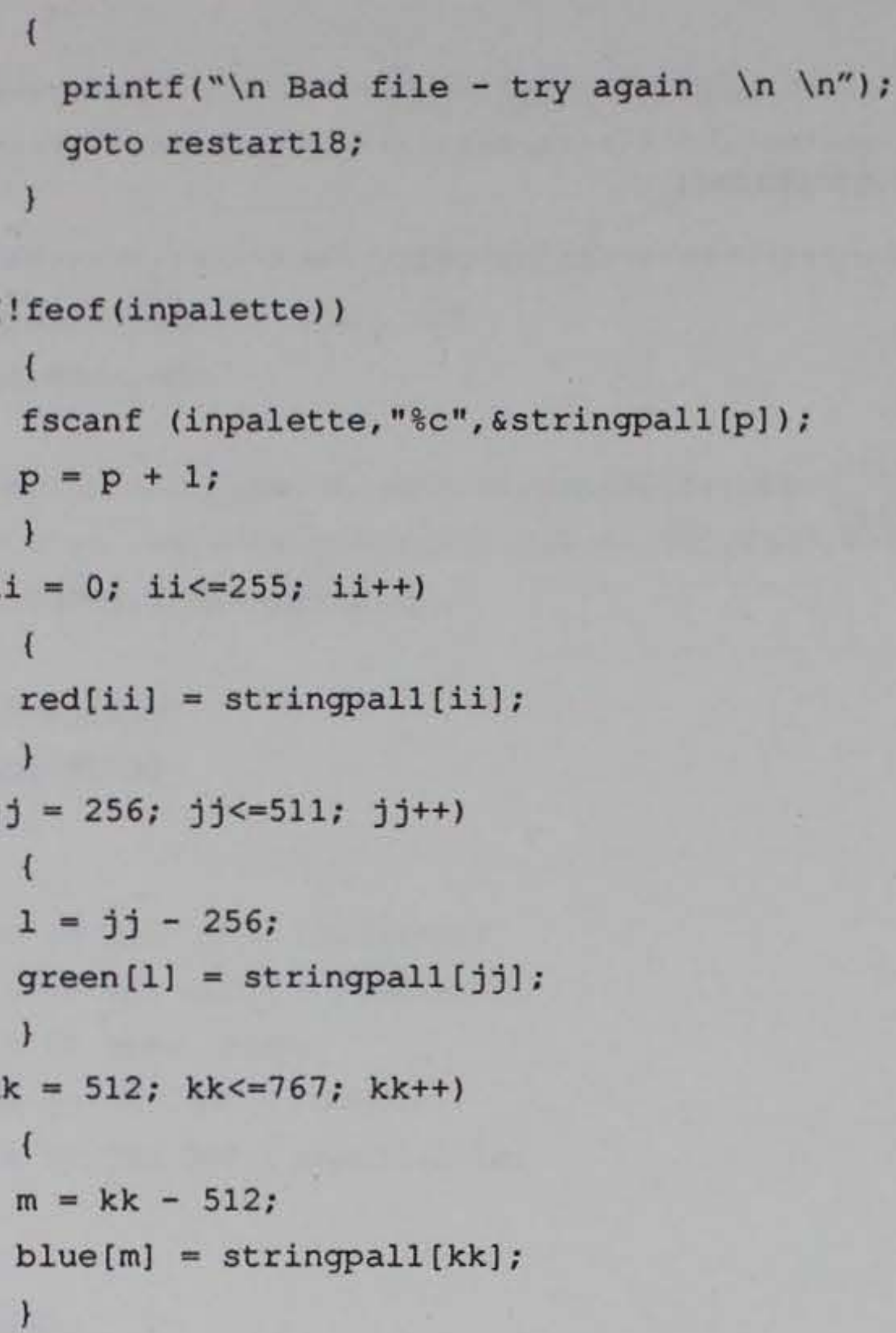




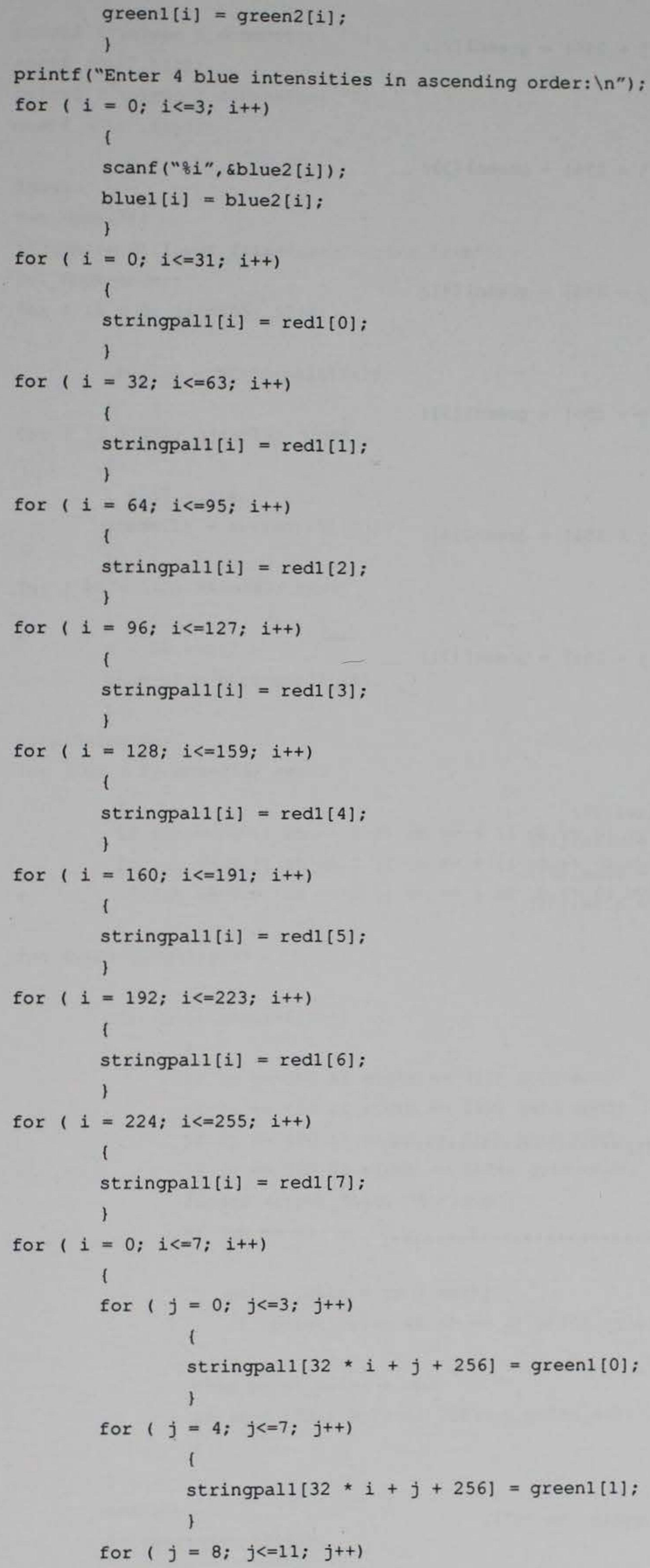




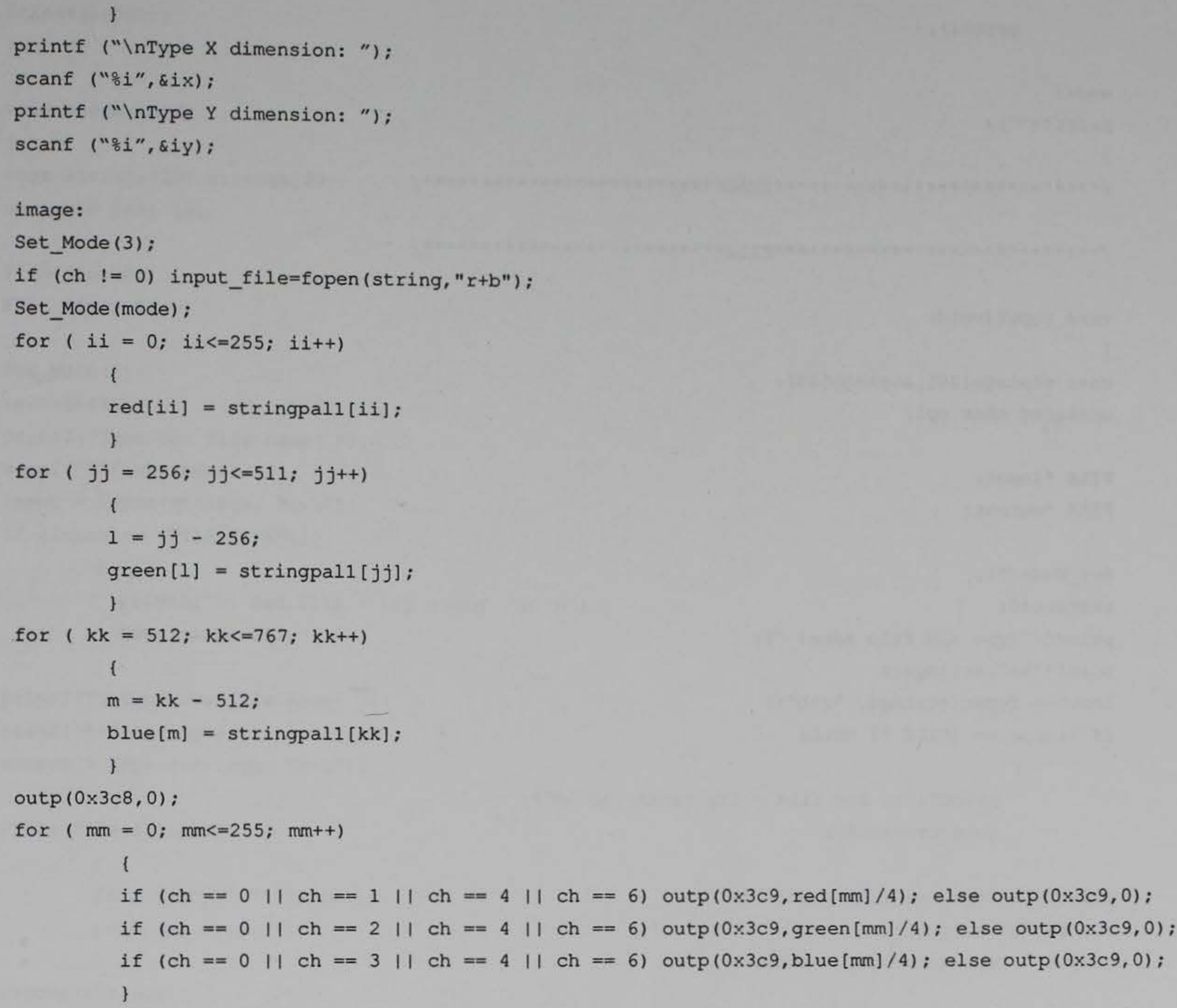


getch () ;

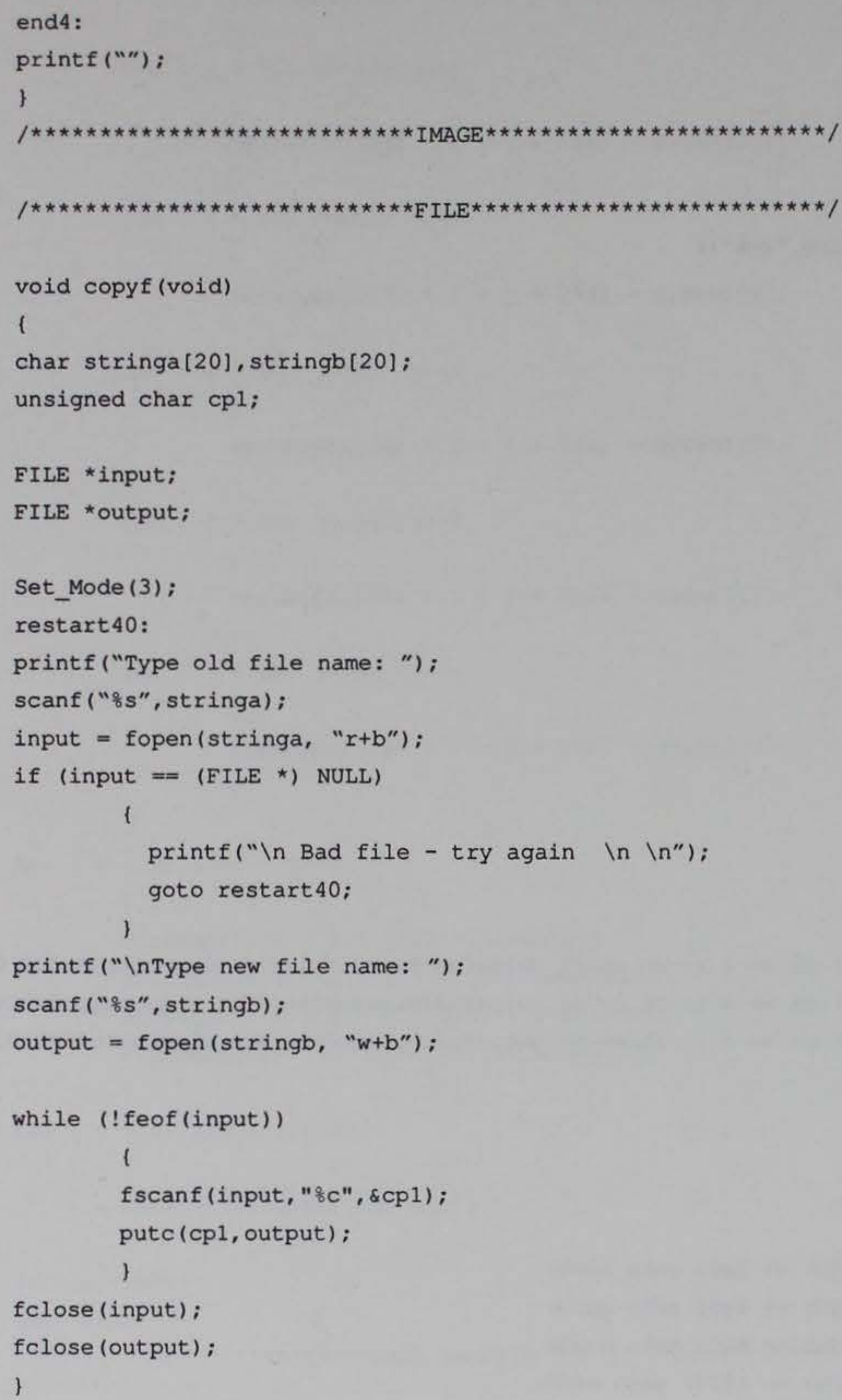



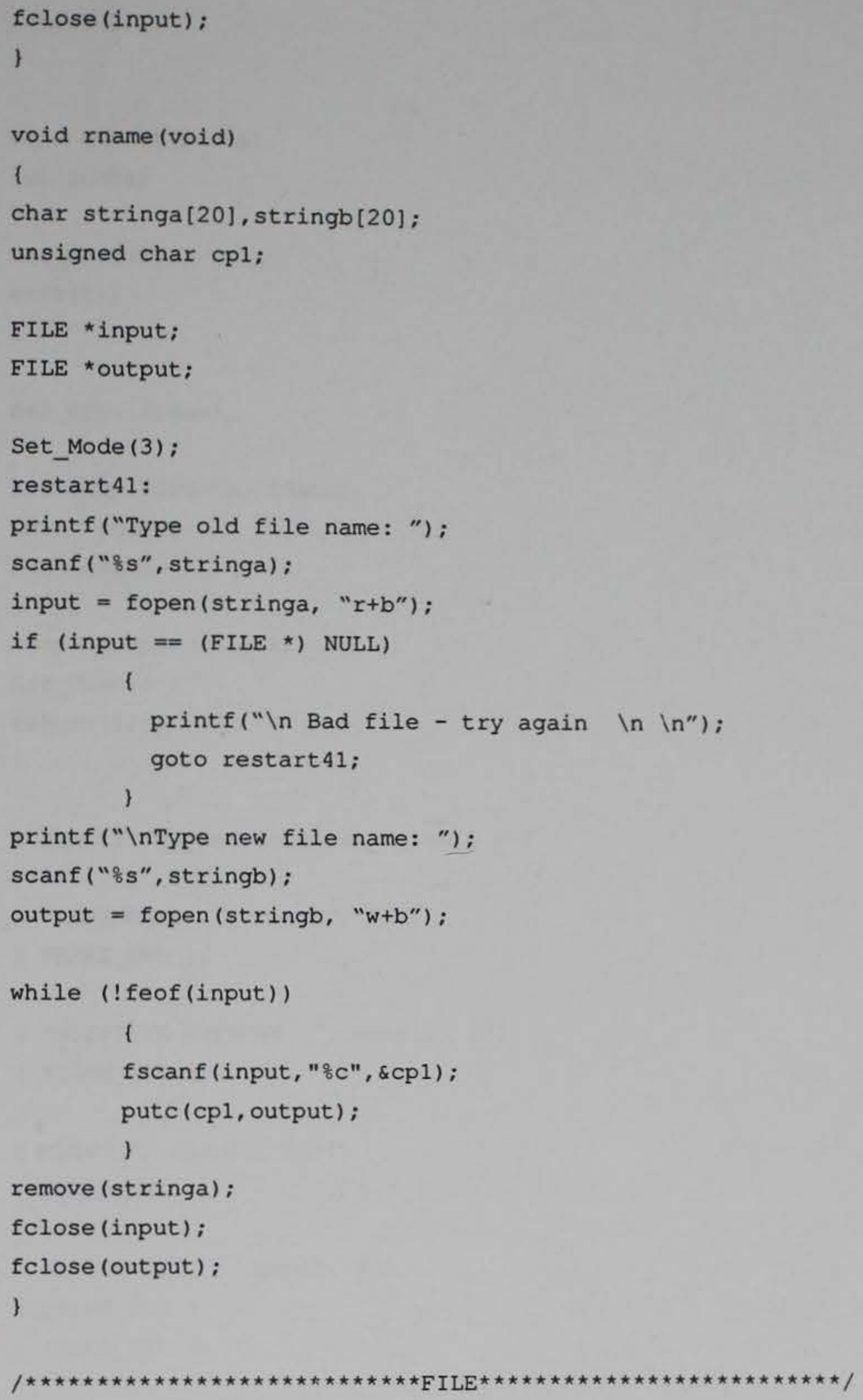

int metro(sdata, data)

char * sdata;

int data;

1

int mask, ch;

mask $=255 ;$

$\mathrm{ch}=0$;

if (data $==23) \mathrm{ch}=1$;

if (data $==24) \mathrm{ch}=2$;

if (data $==25) \mathrm{ch}=3$;

if (data $==26$ )

1

$\mathrm{ch}=4 ;$ 


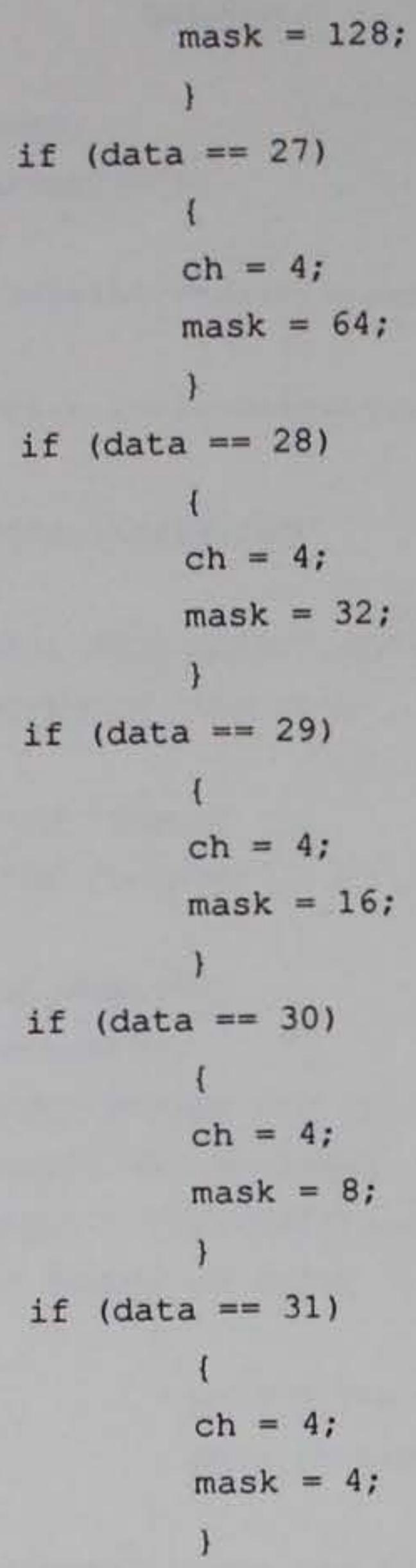




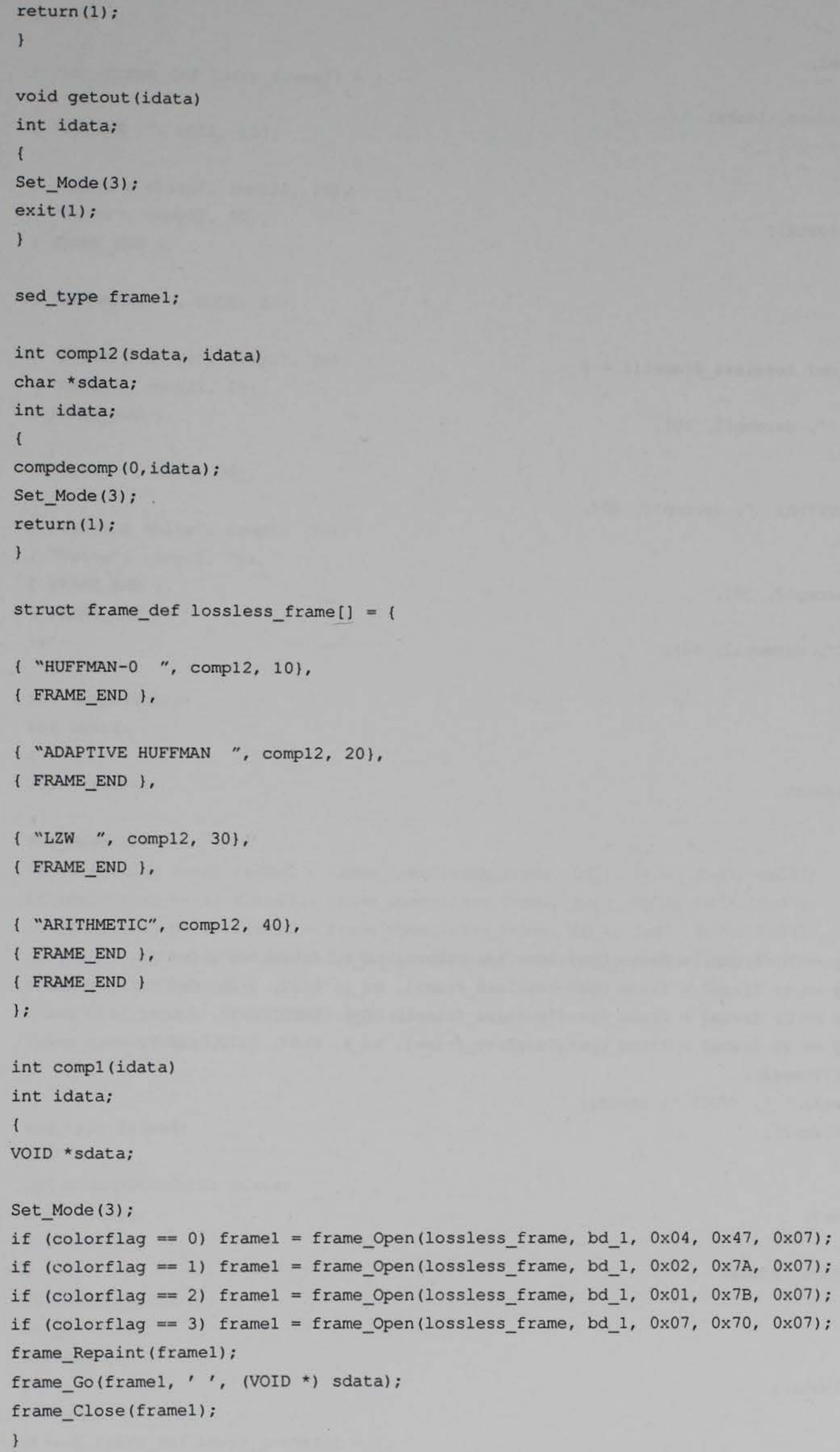




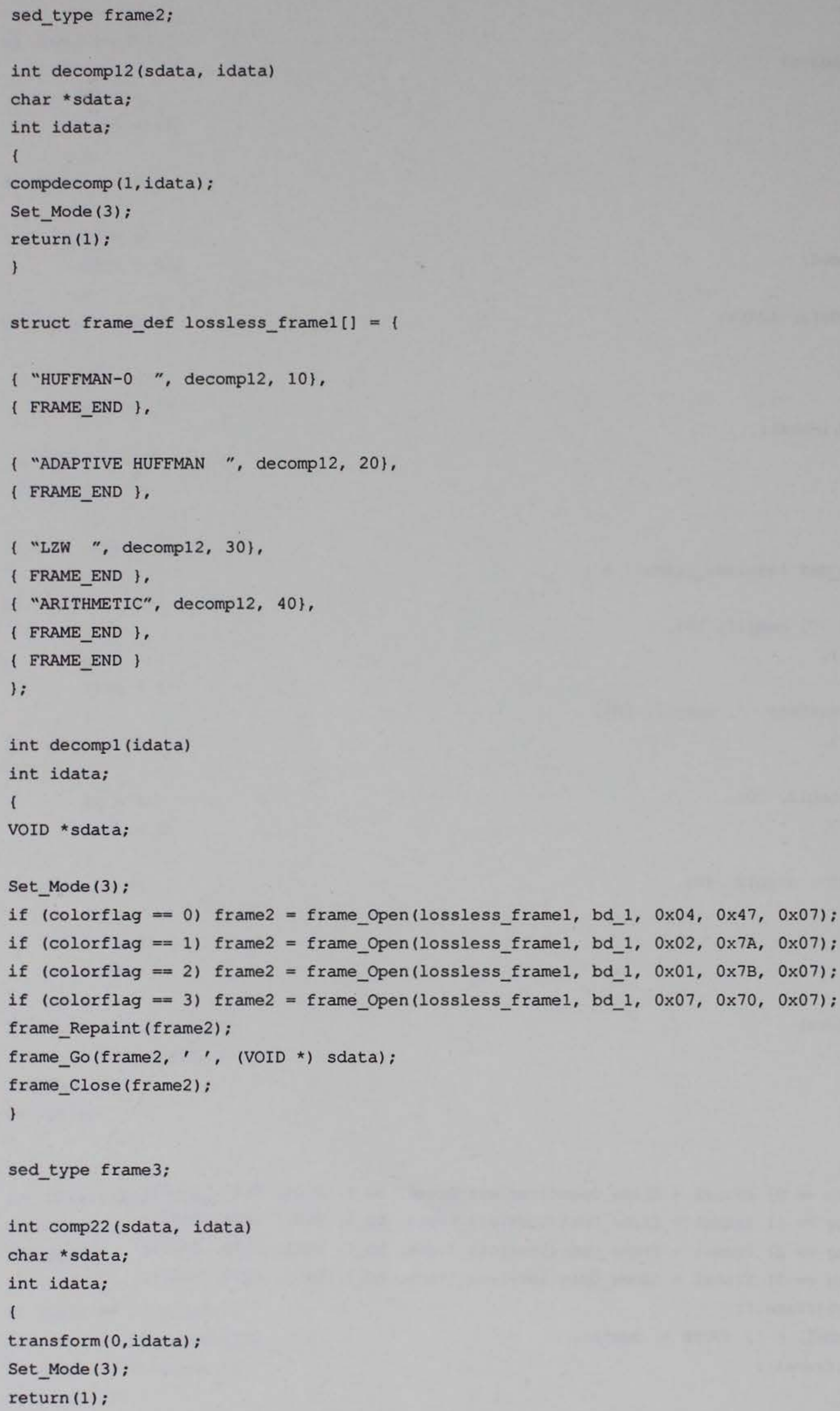




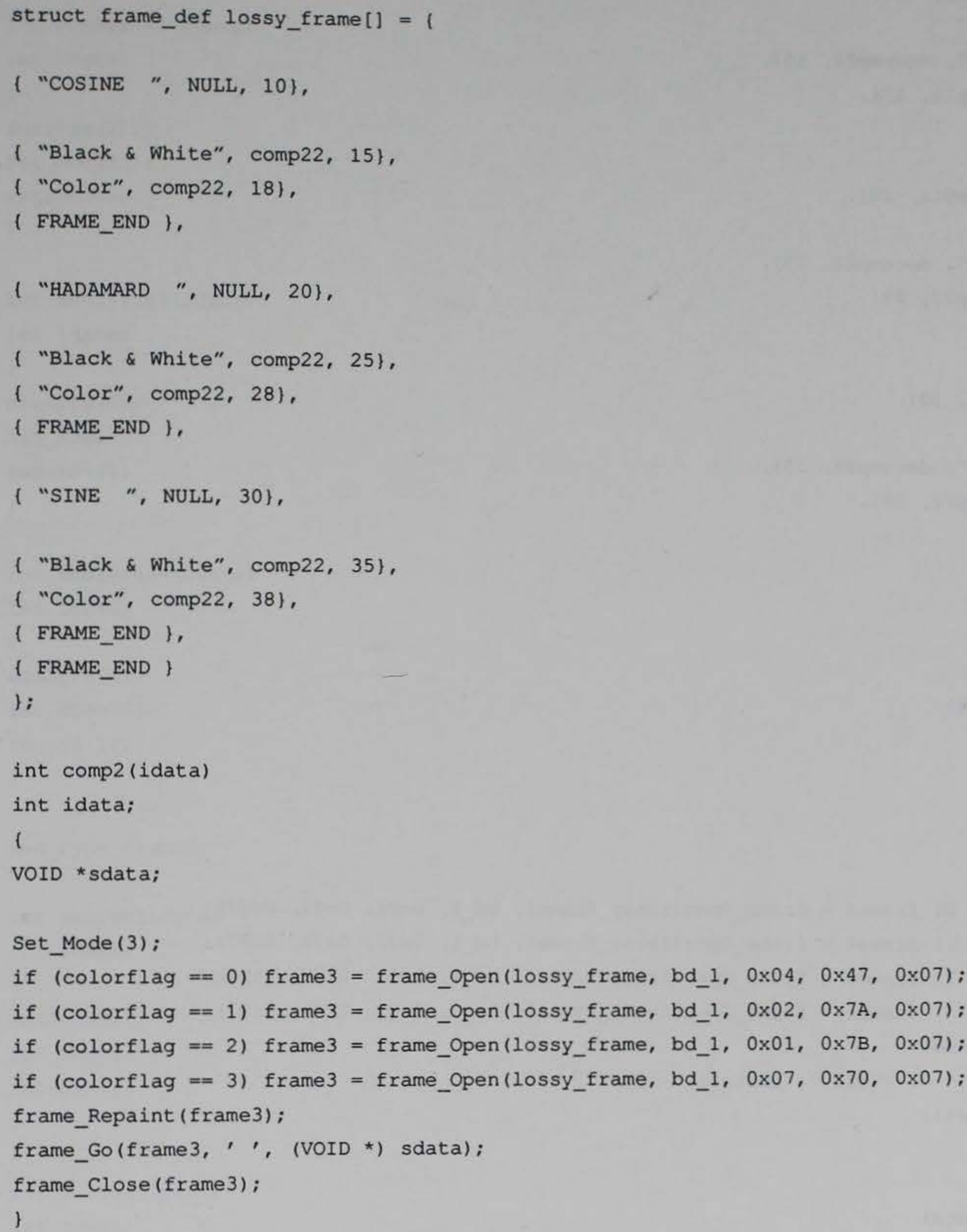




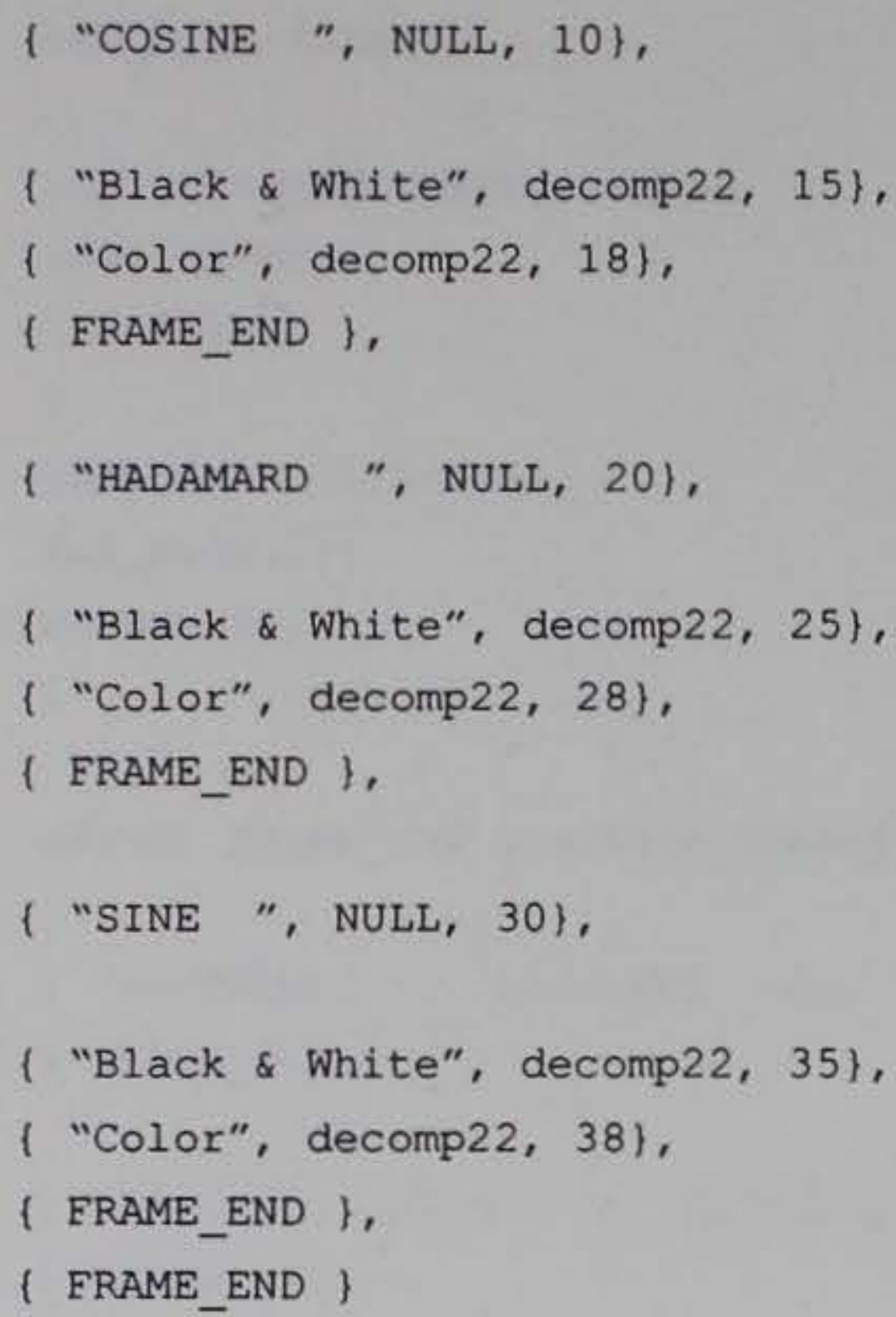


int analysis2(idata)

int idata;

1

analyze (2) ;

Set_Mode (3) ;

return (1);

)

int analysis3 (idata)

int idata;

1

analyze (3) :

Set_Mode (3) ;

return (1) ;

)

int analysis4(idata)

int idata;

i

analyze (4) ;

Set_Mode (3) ;

return (1);

)

sed_type frame5;

int pasteh (idata)

int idata;

i

analyze (6) :

Set_Mode (3) ;

return (1) ;

)

int pastev (idata)

int idata;

i

analyze (7) ;

Set_Mode (3) ;

return (1) ;

)

struct frame_def chop_framel[] $=1$

$\{$ "hORIZONTALly ", pasteh, 10\},

$\{$ FRAME_END $\}$,

$\{$ "Vertically ", pastev, 20\},

$\{$ FRAME_END \}, 


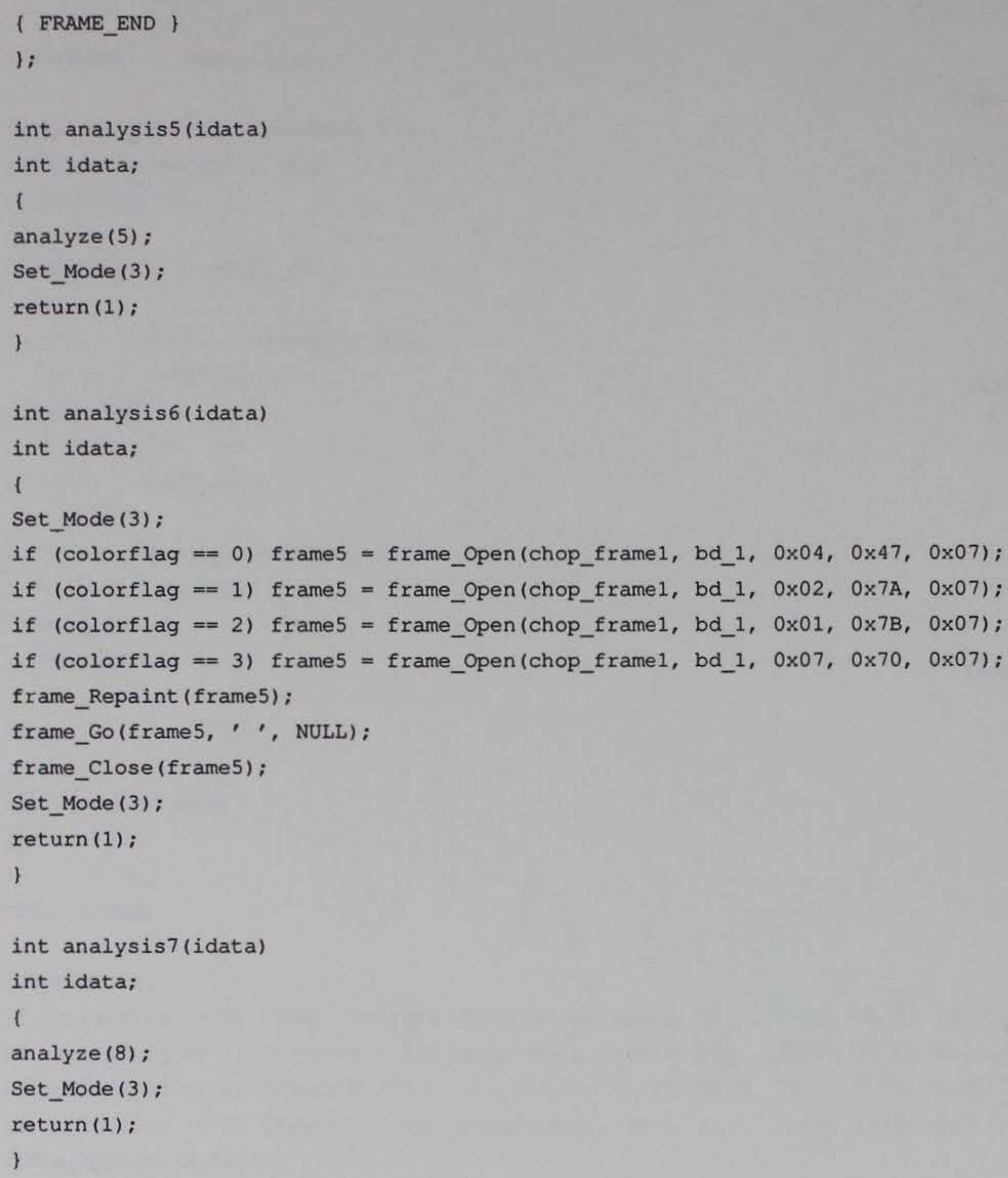




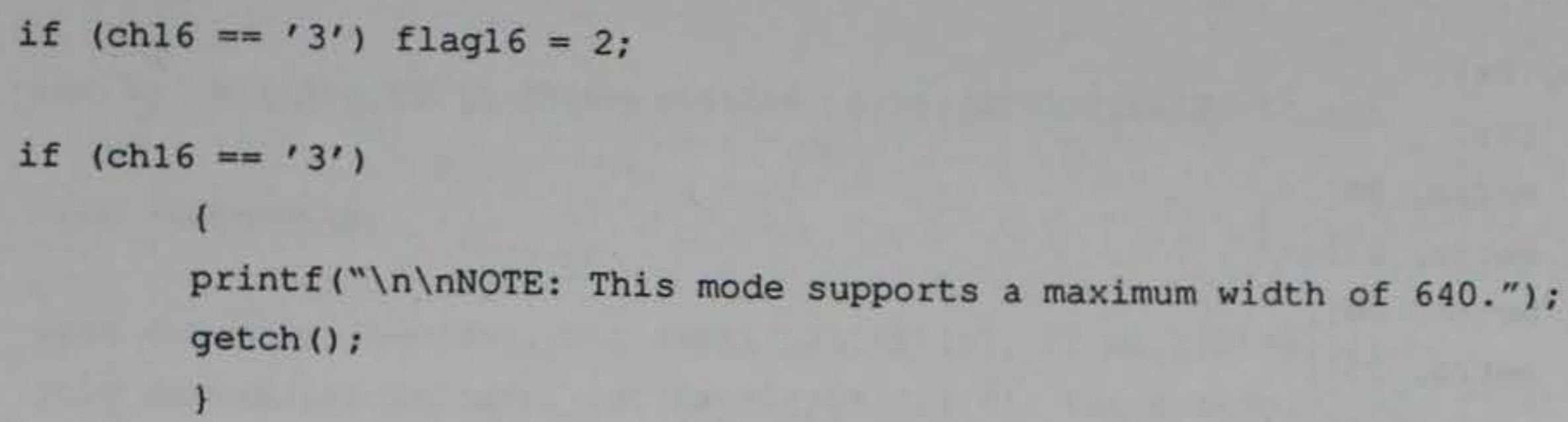




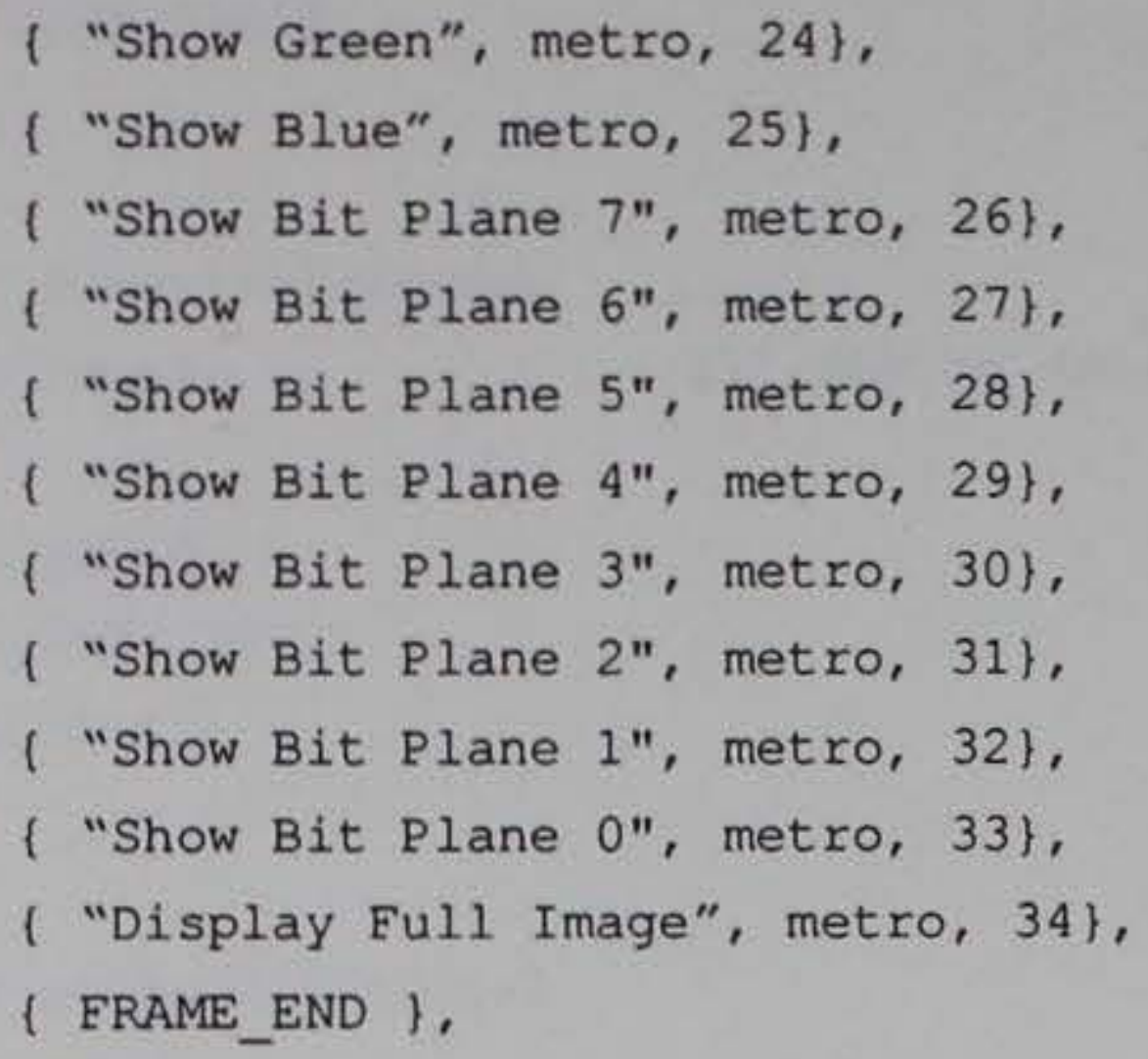




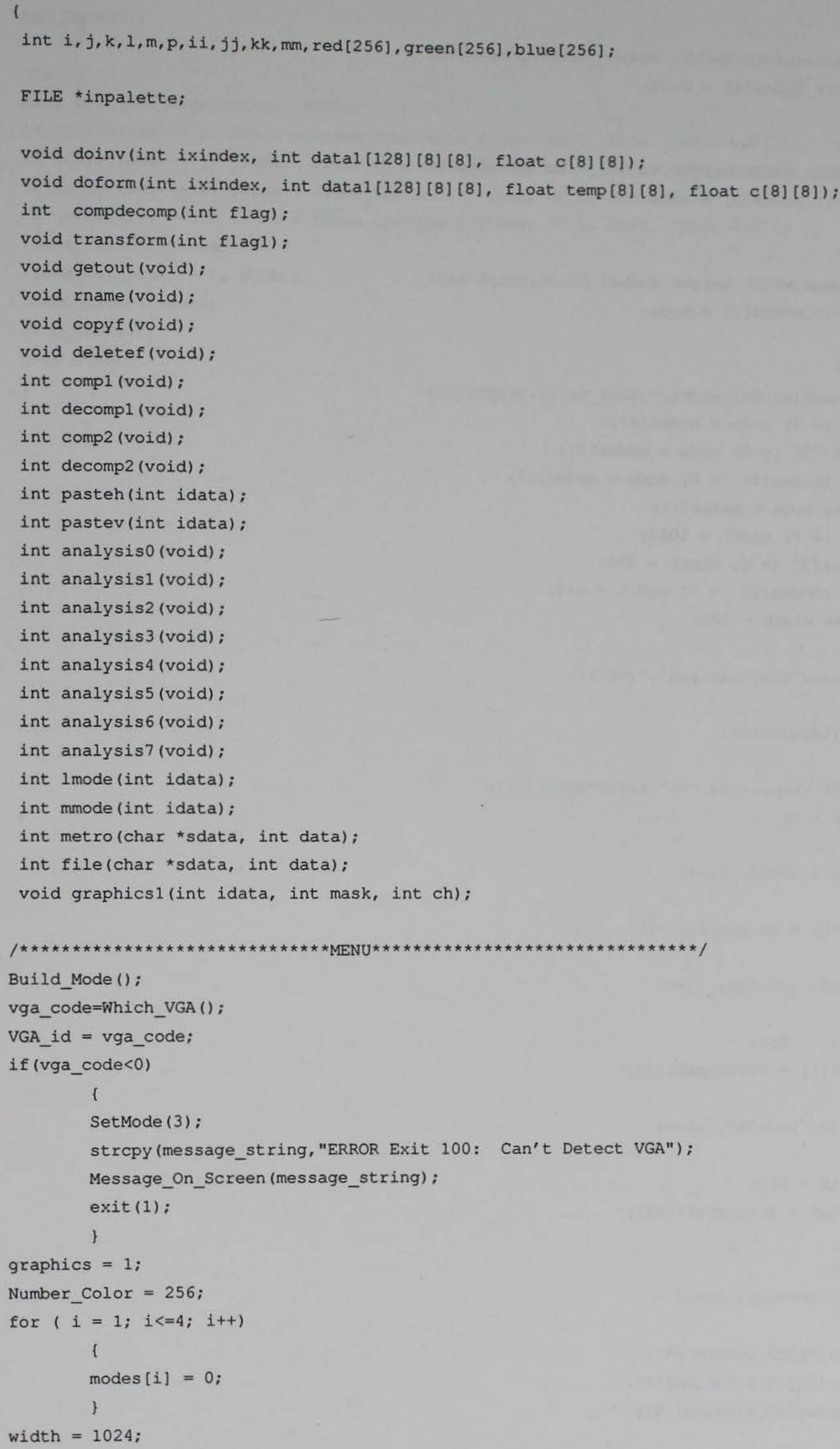




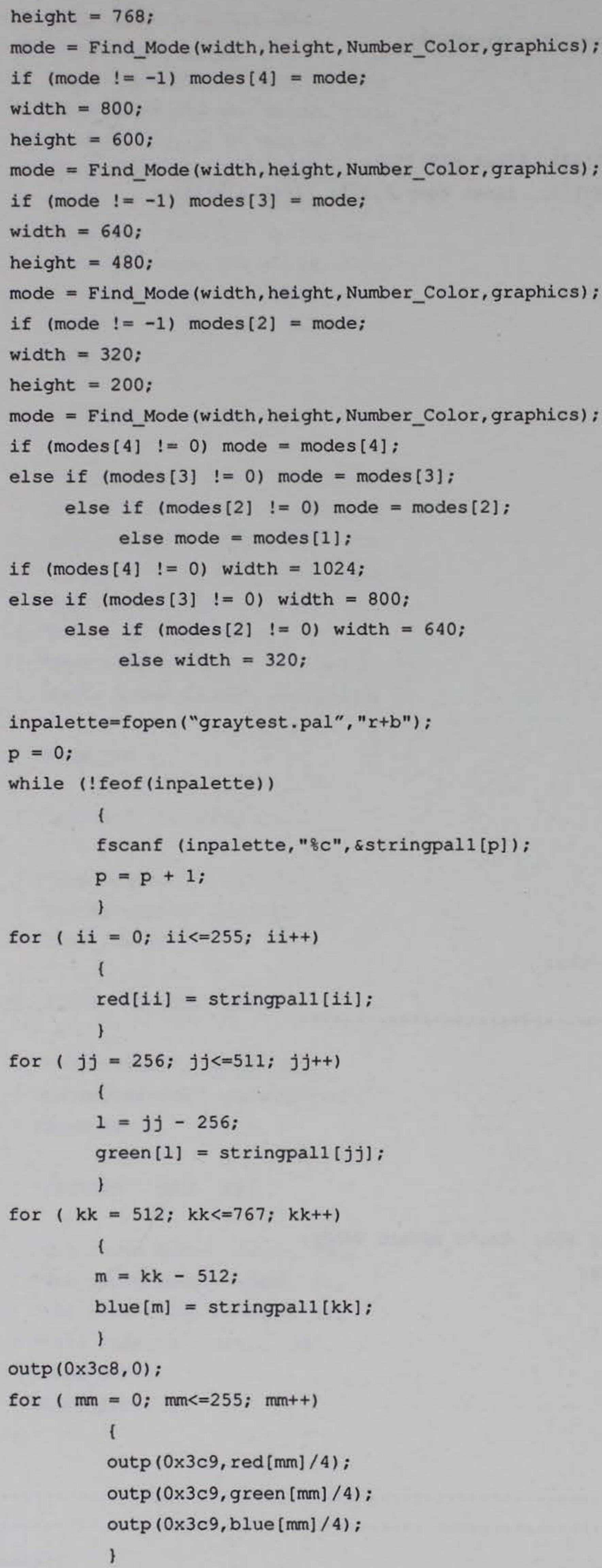


Set_Mode (3) ;

start:

disp_Init(def_ModeCurrent, NULL);

if $($ colorflag $==0$ ) frame $=$ frame_Open (main_frame, bd_1, 0x04, 0x47, 0x07);

if $($ colorflag $==1)$ frame $=$ frame_Open (main_frame, bd_1, 0x02, 0x7A, 0x07);

if $(\operatorname{colorflag}==2)$ frame $=$ frame_Open (main_frame, bd_1, 0x01, 0x7B, 0x07);

if (colorflag $==3$ ) frame $=$ frame_Open (main_frame, bd_1, 0x07,0x70,0x07);

frame_Repaint (frame);

frame_Go (frame, ' ' , NULL) ;

frame_Close (frame) ;

disp_Close () ;

goto start;

\} 
LABX.C

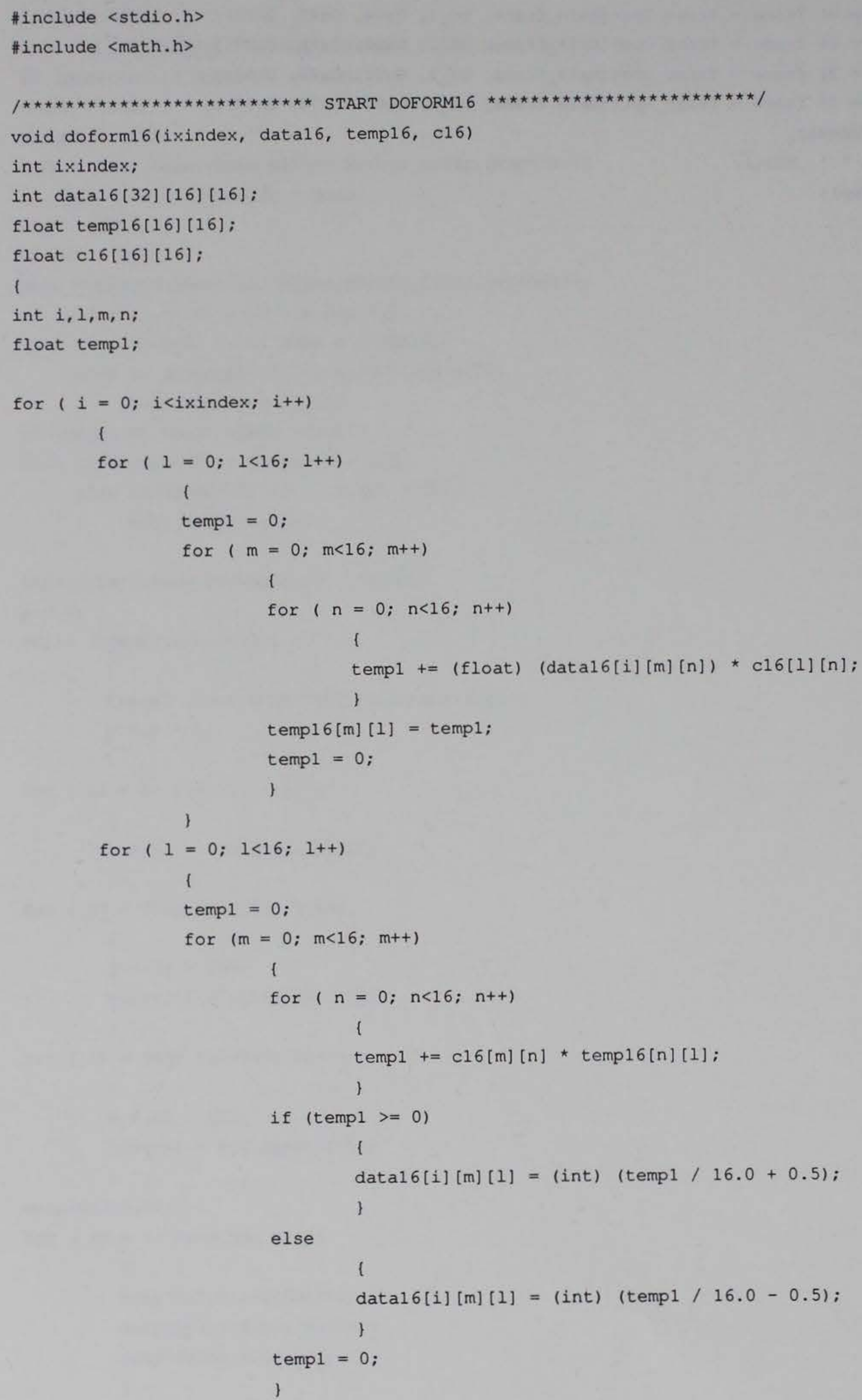


int ixindex;

int data16[32] [16] [16];

float c16[16] [16];

1

int $a, b, q, d$;

float temp1, temp [16][16] ;

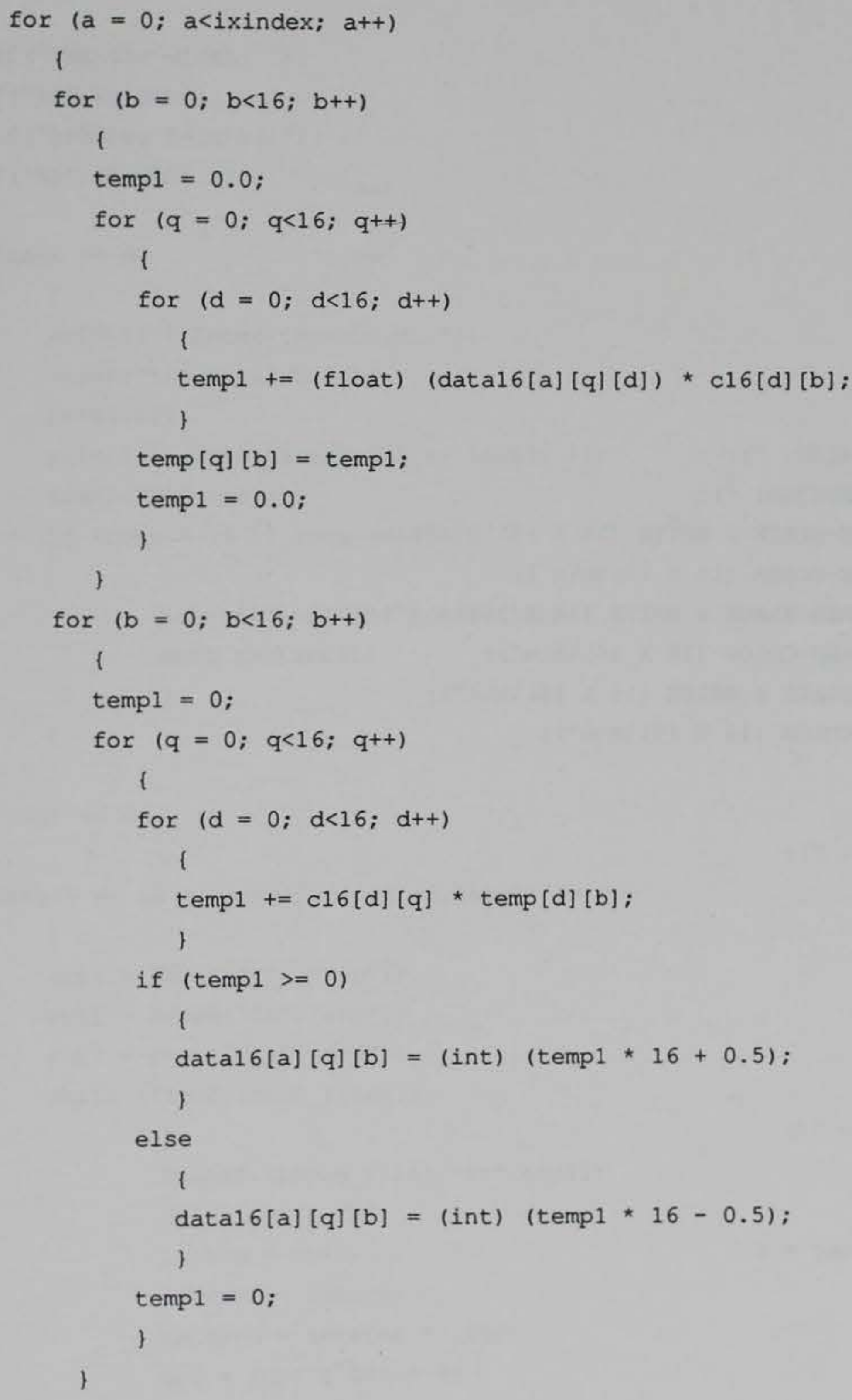




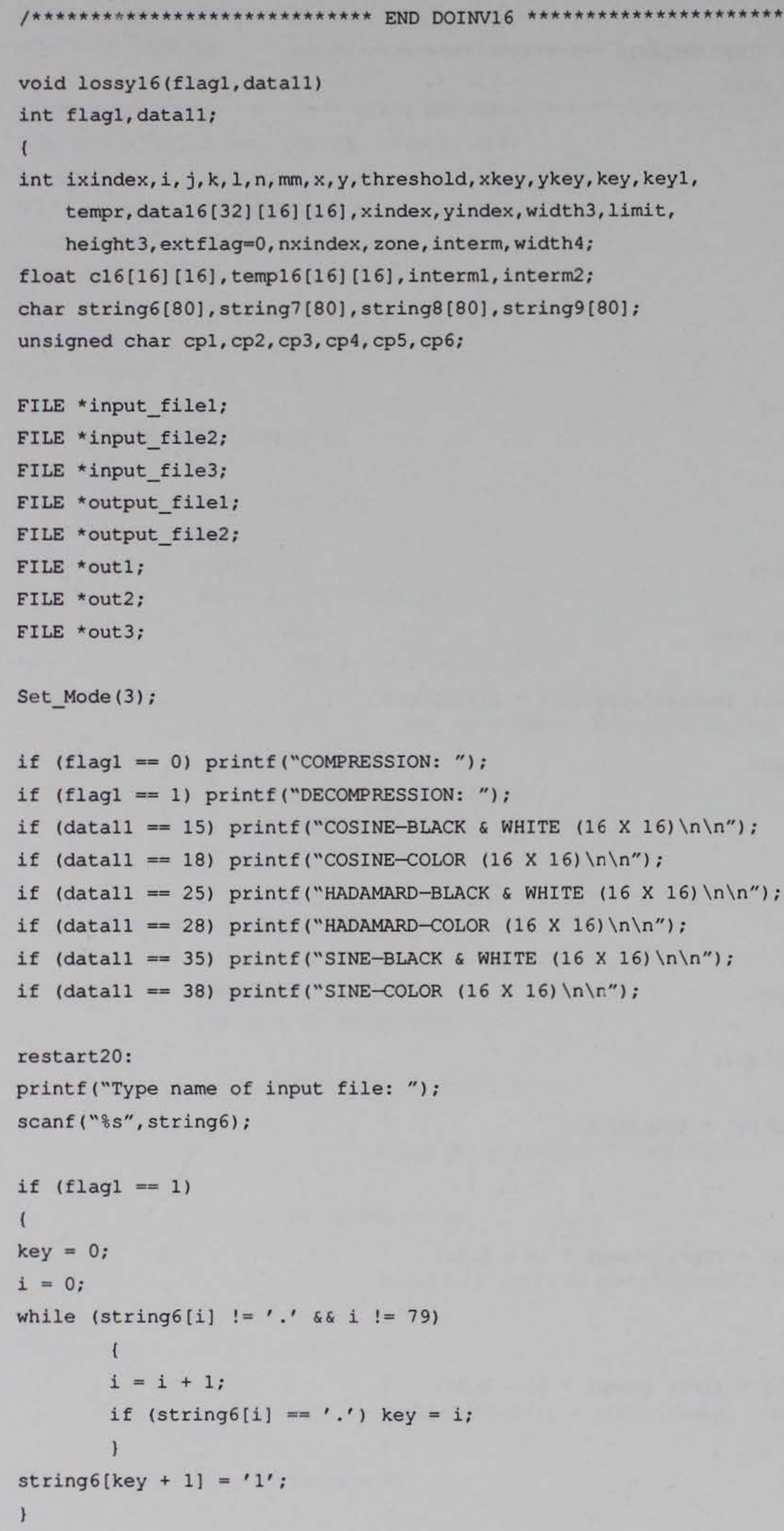




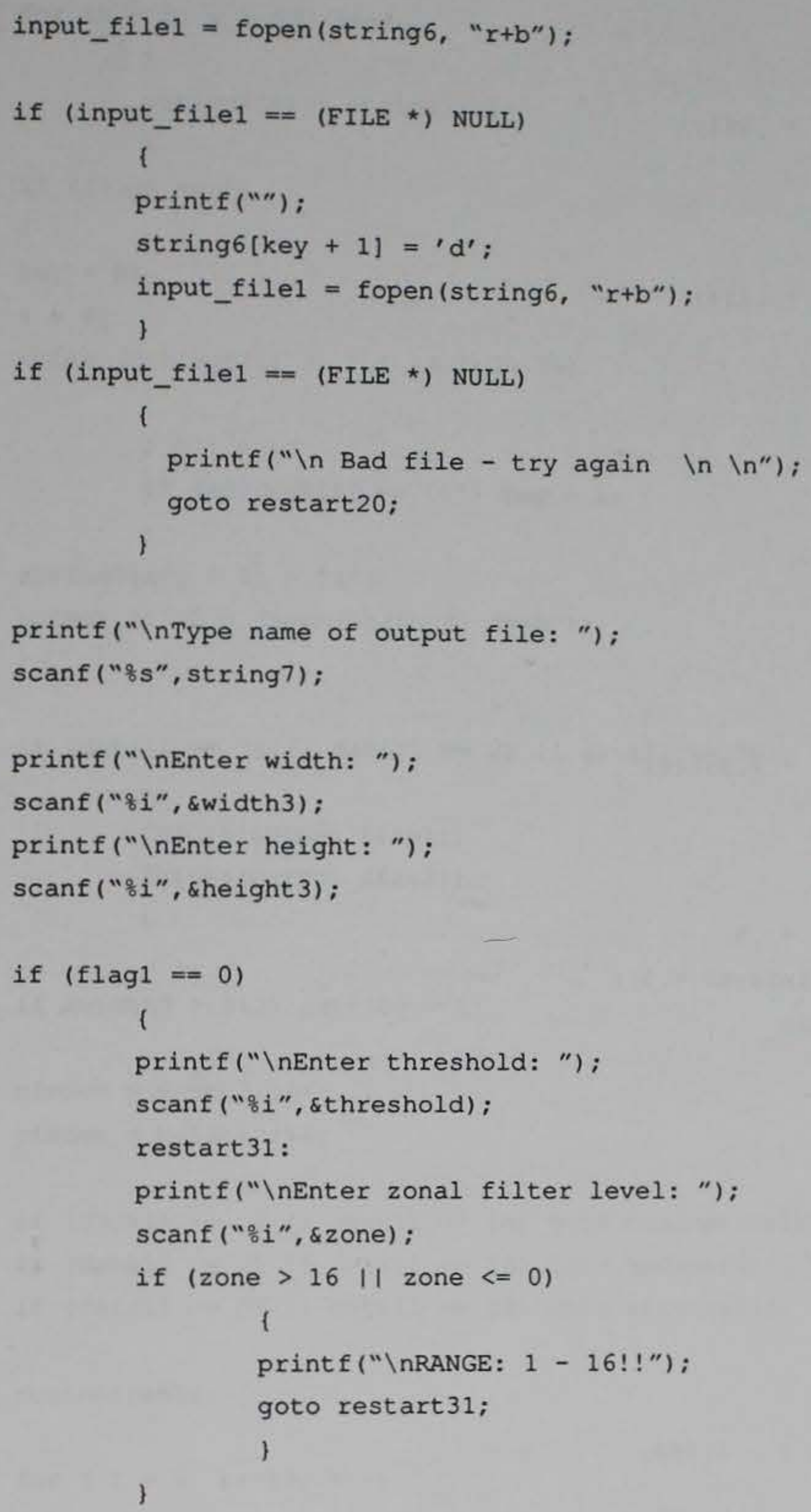




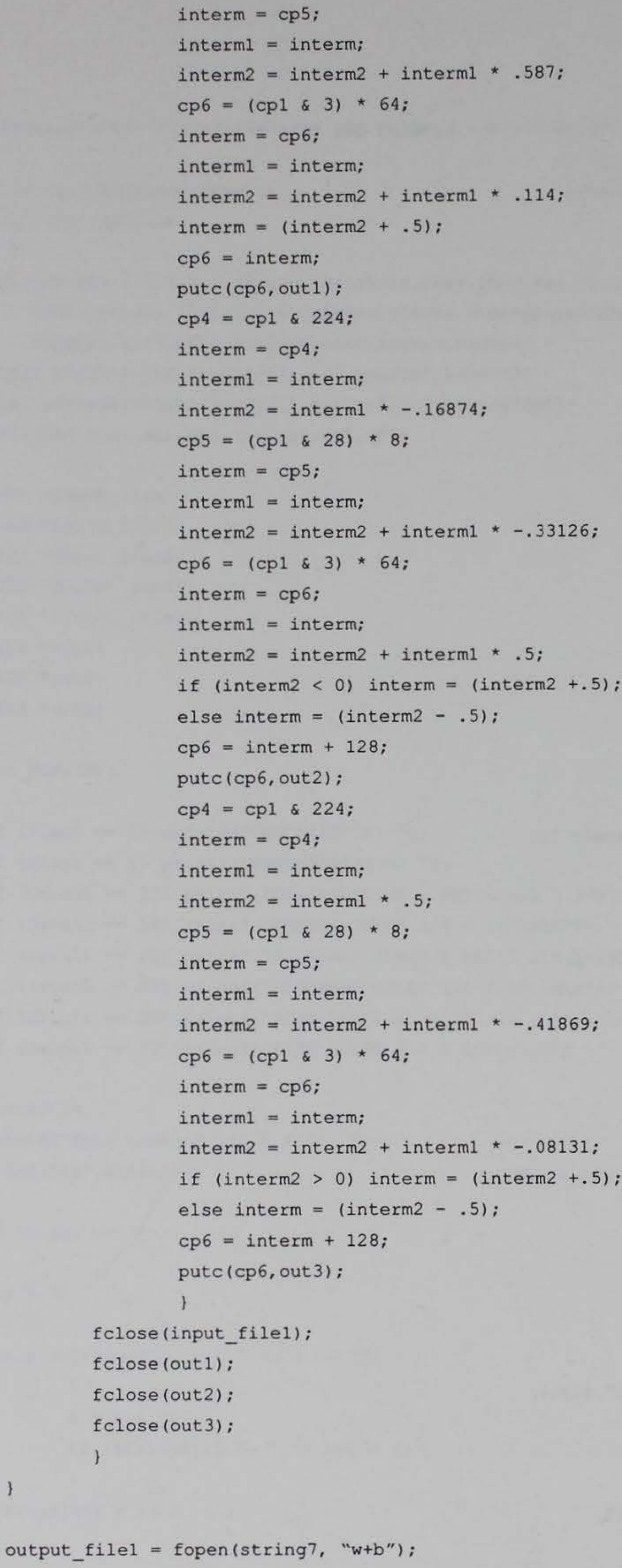




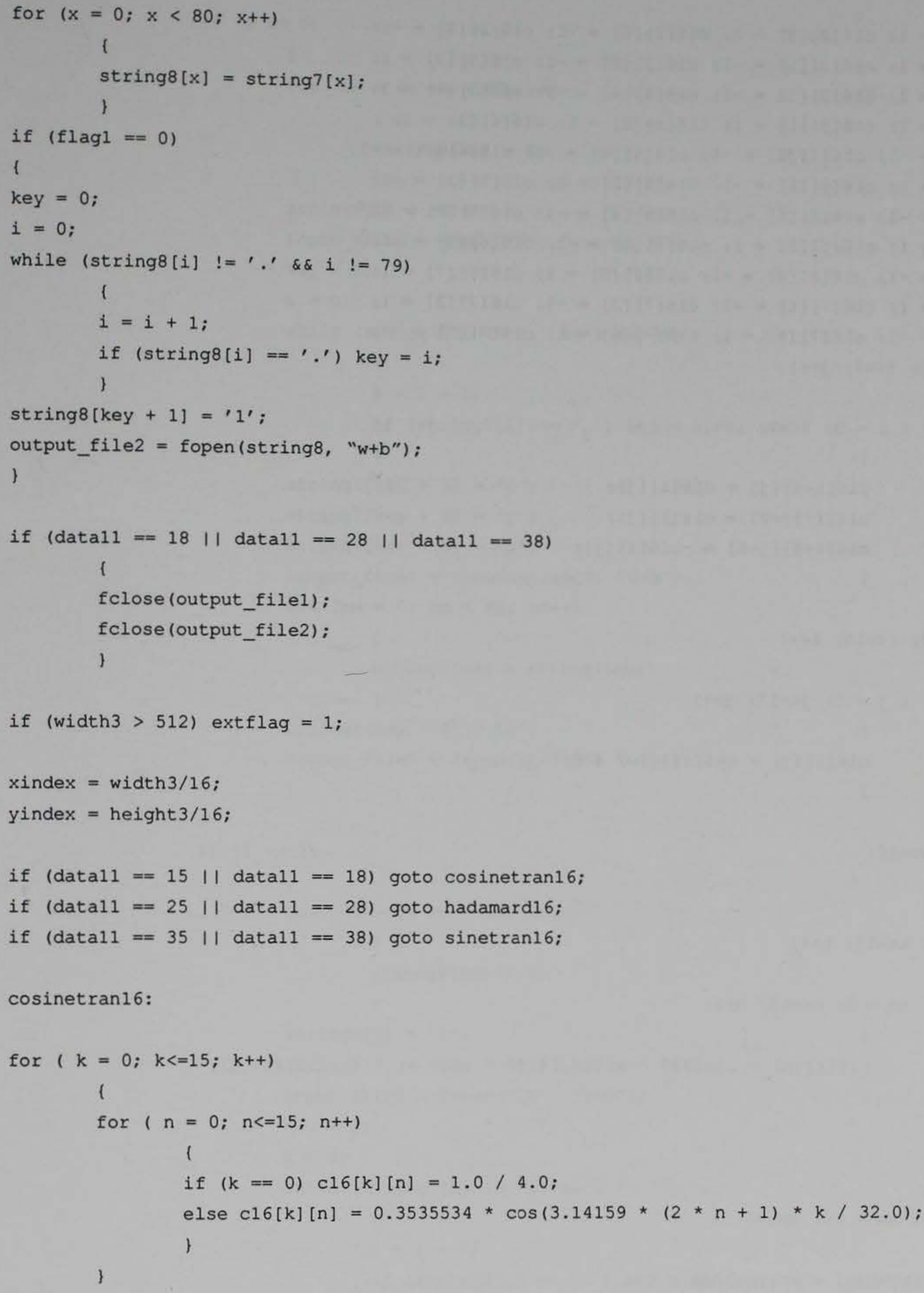




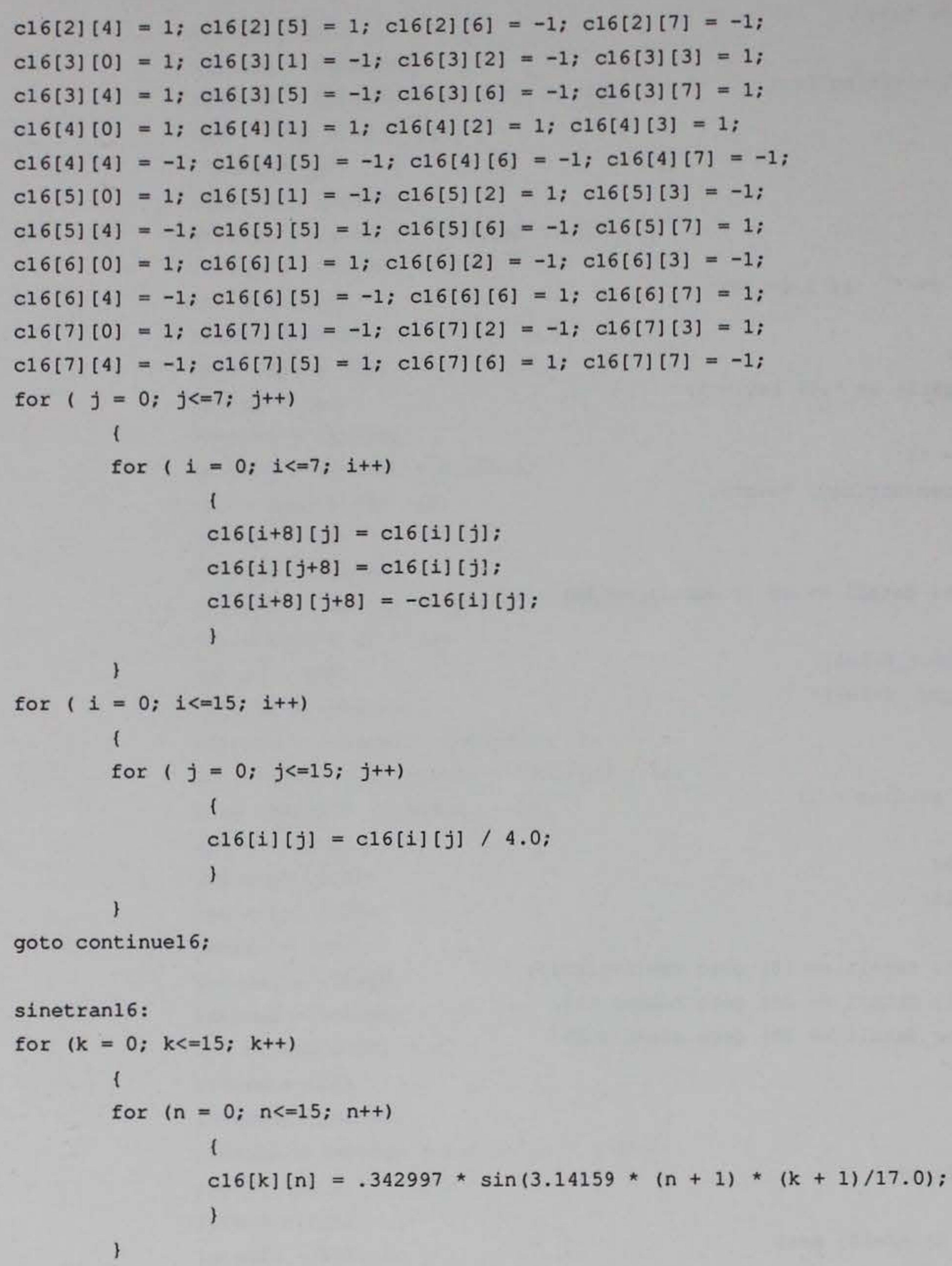




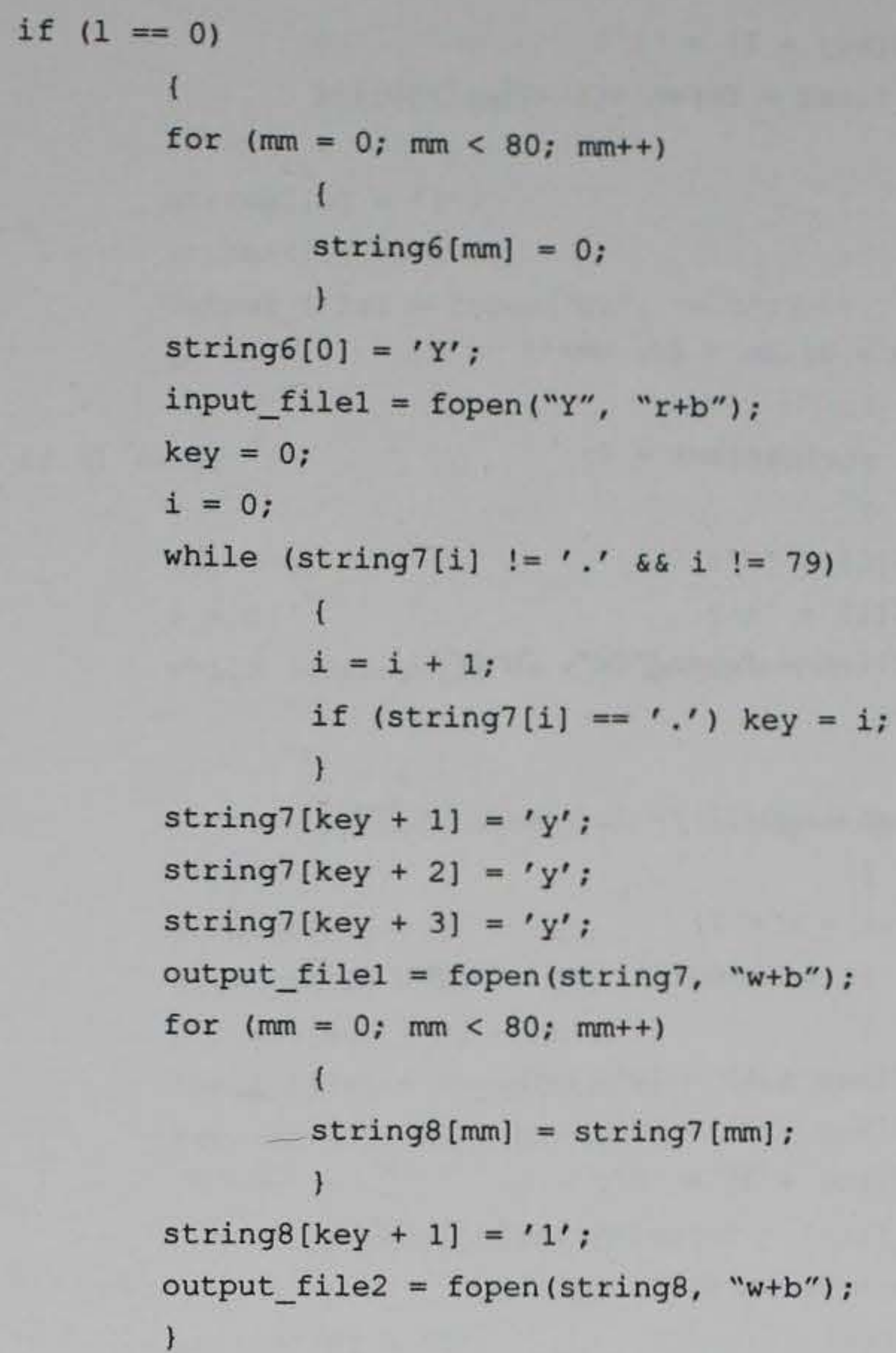




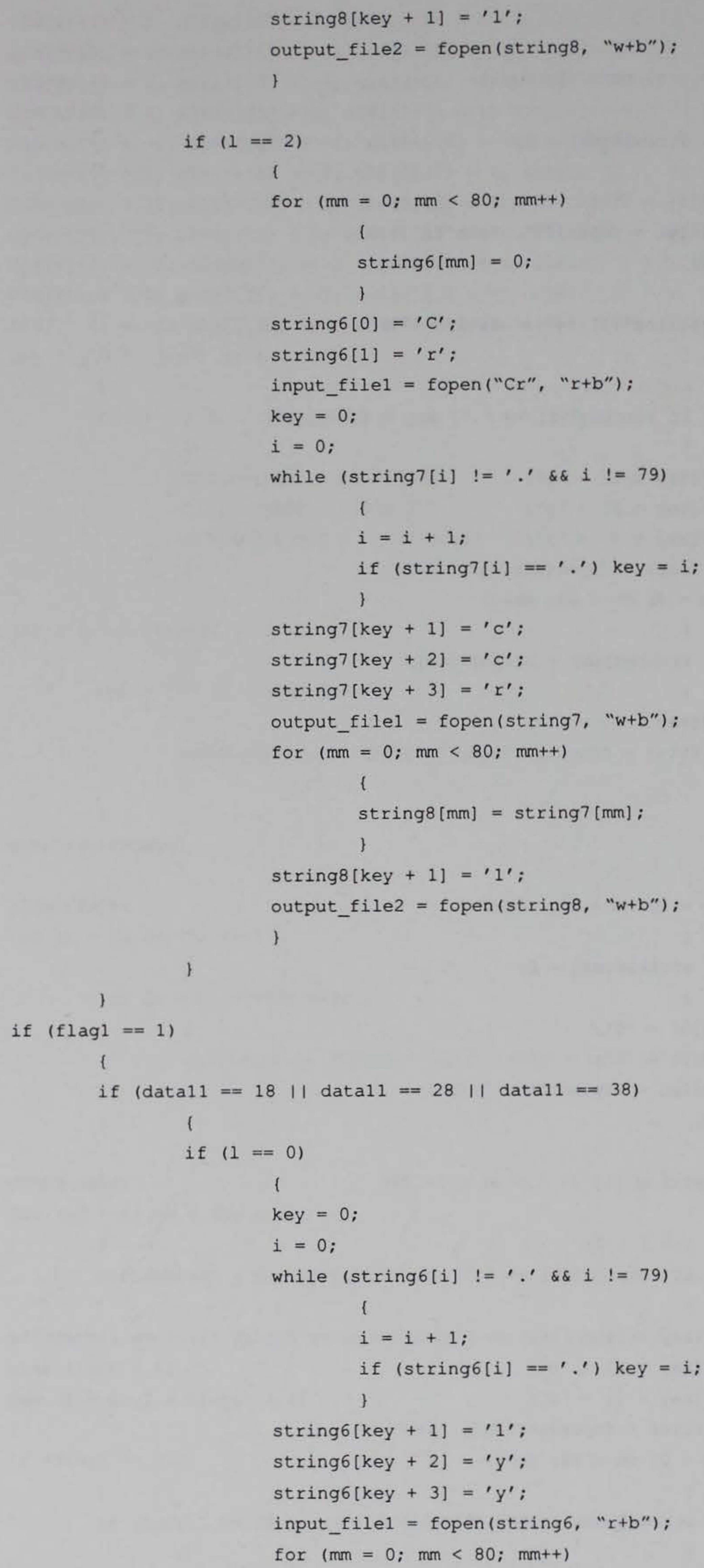




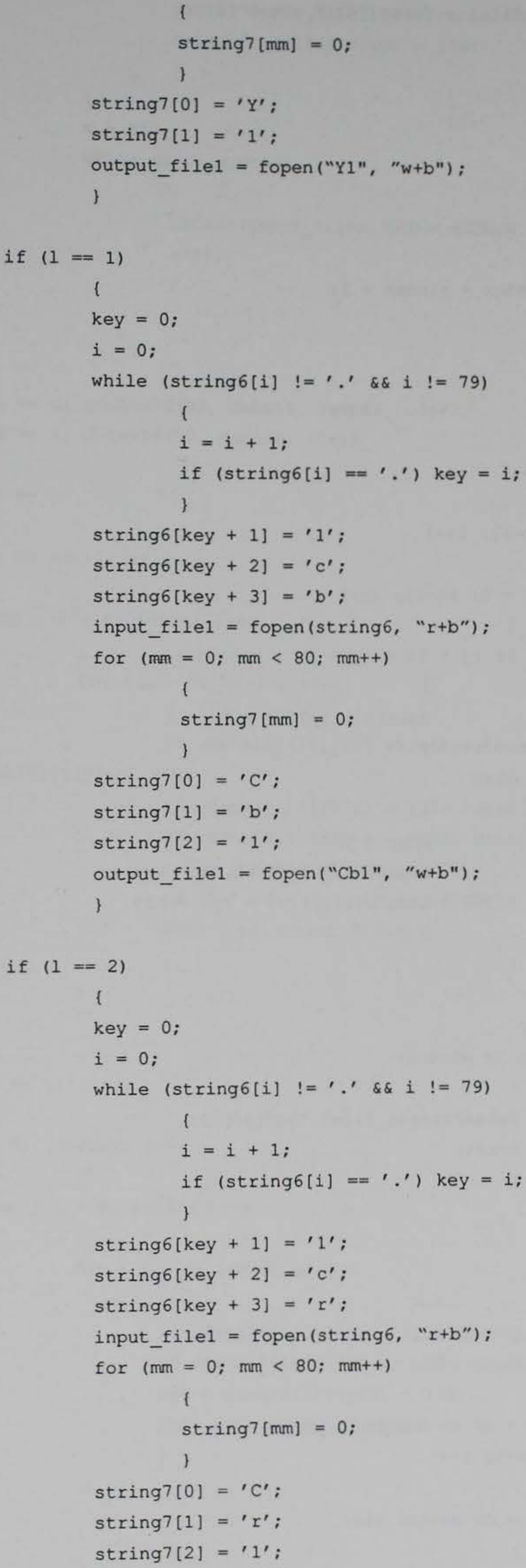




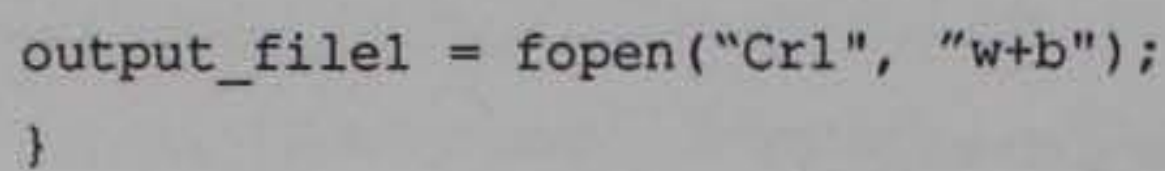




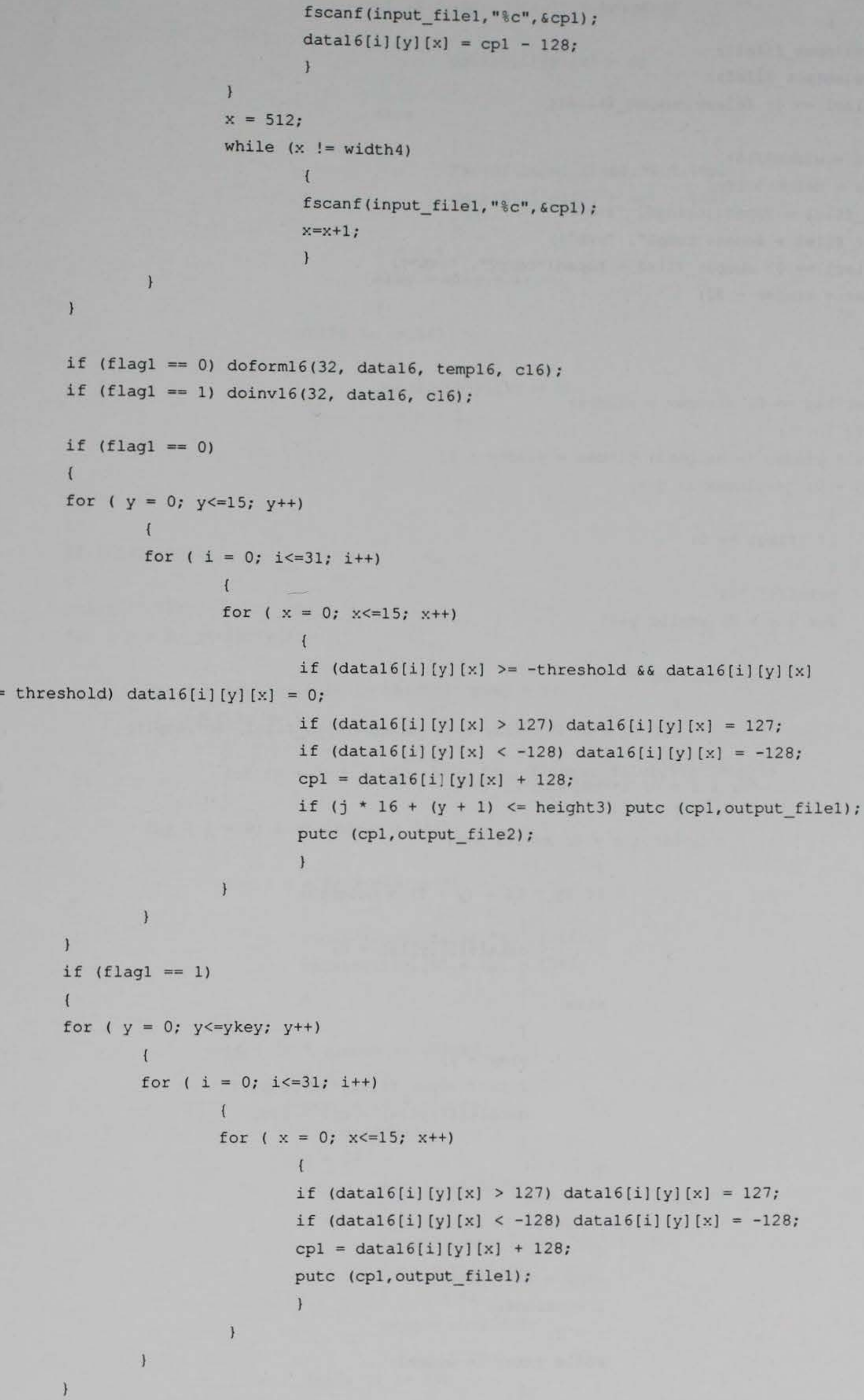




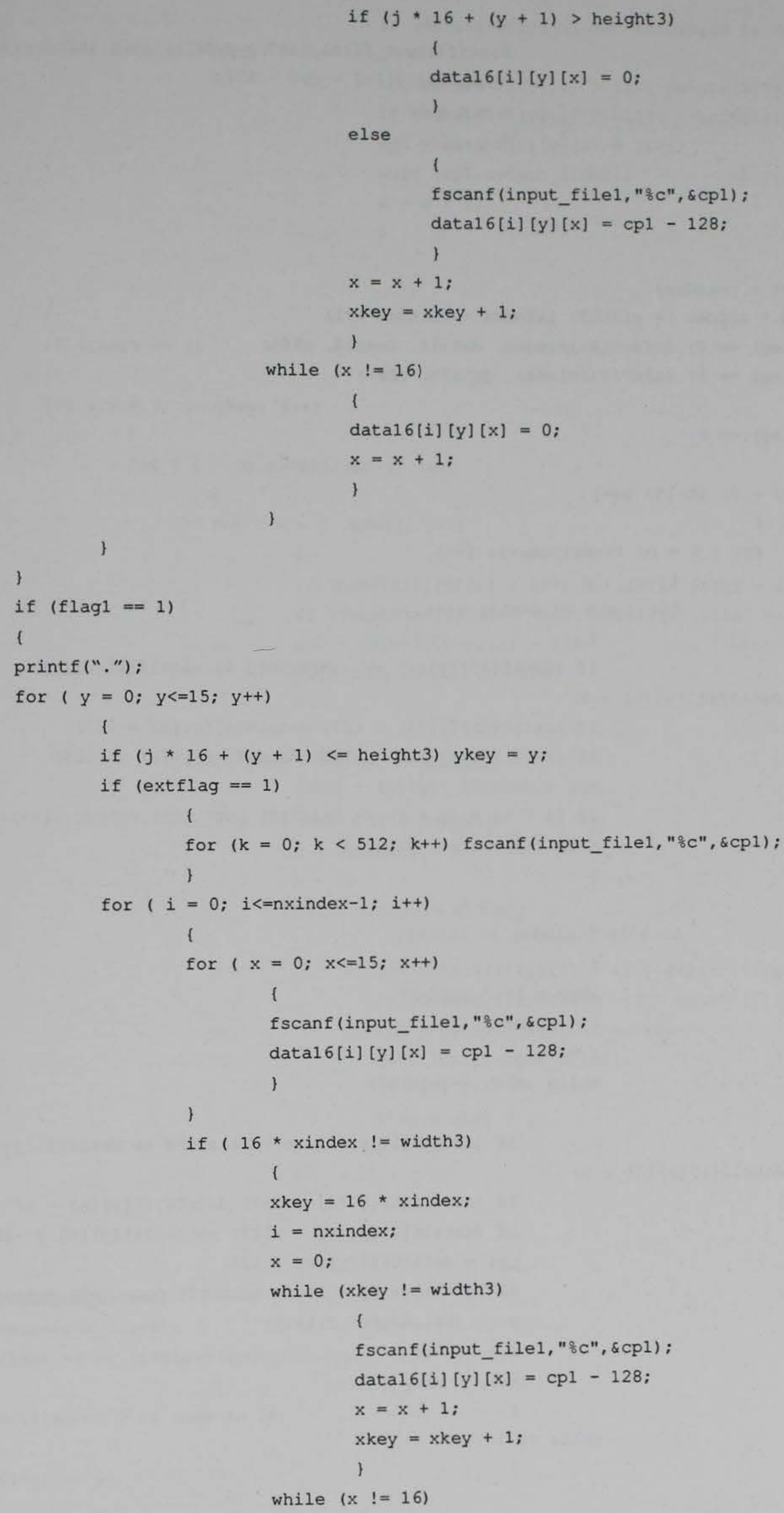




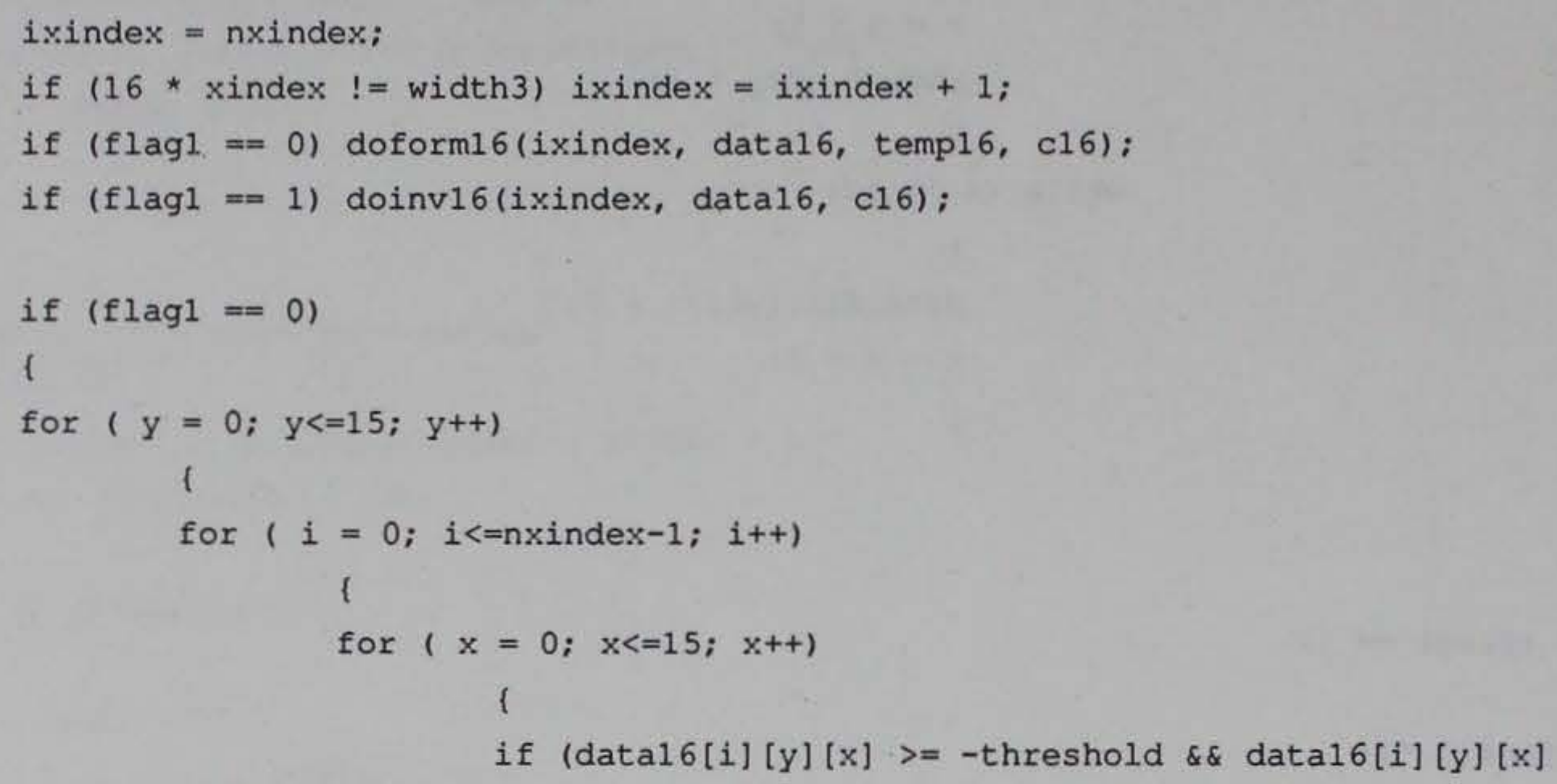


threshold) data16[i][y][x]=0;

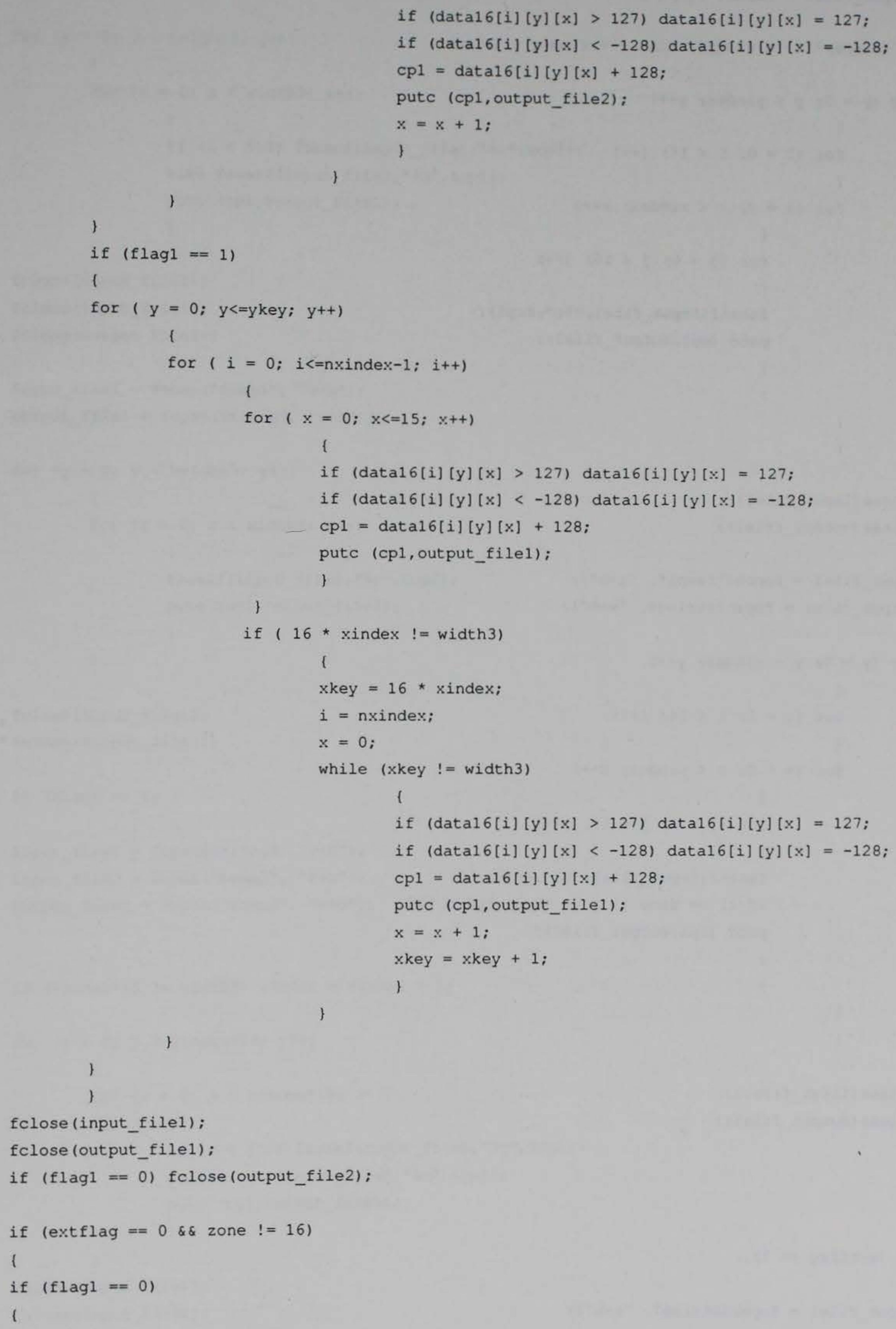




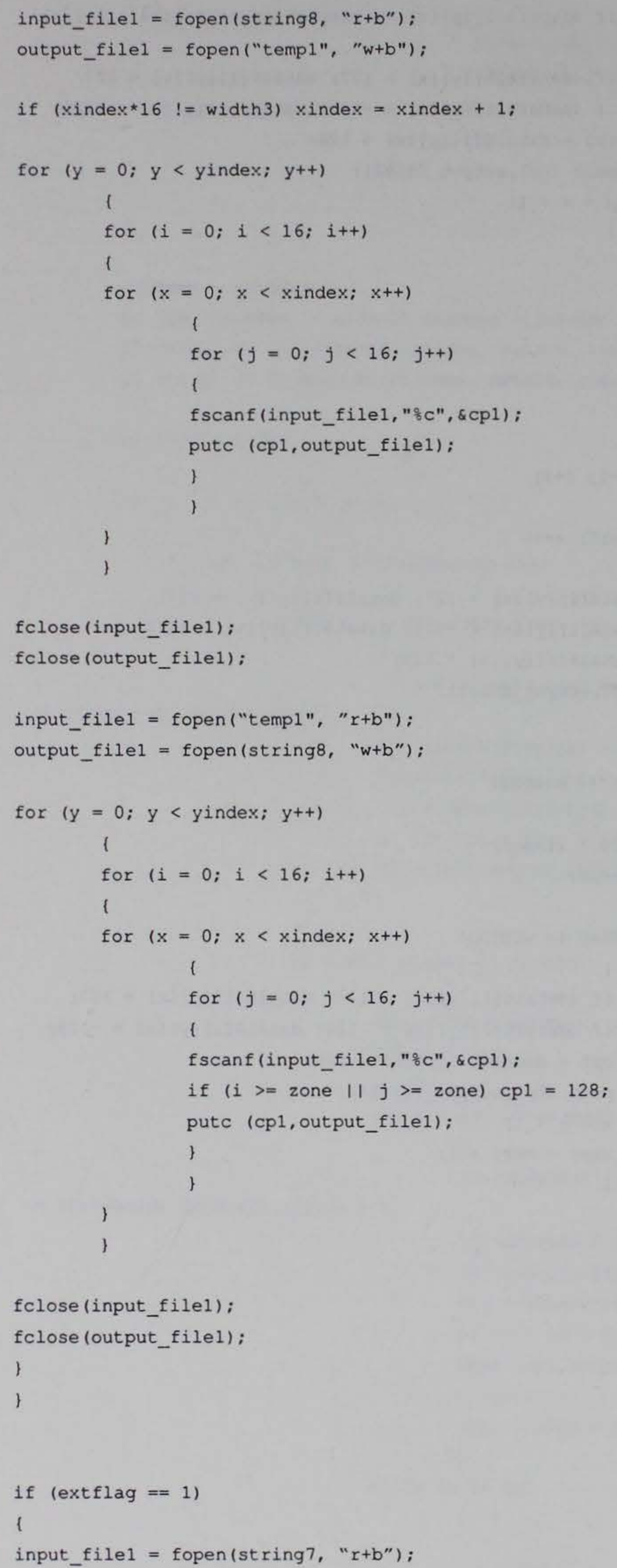




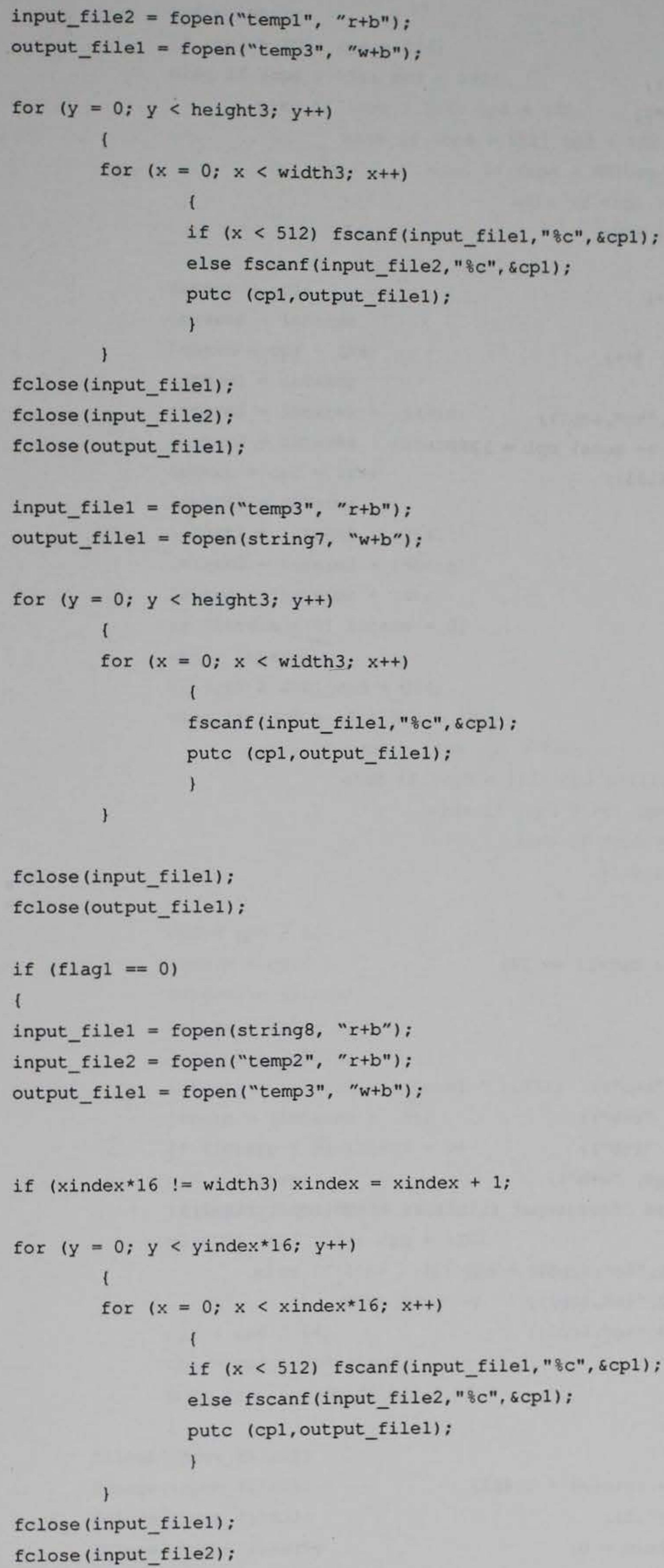




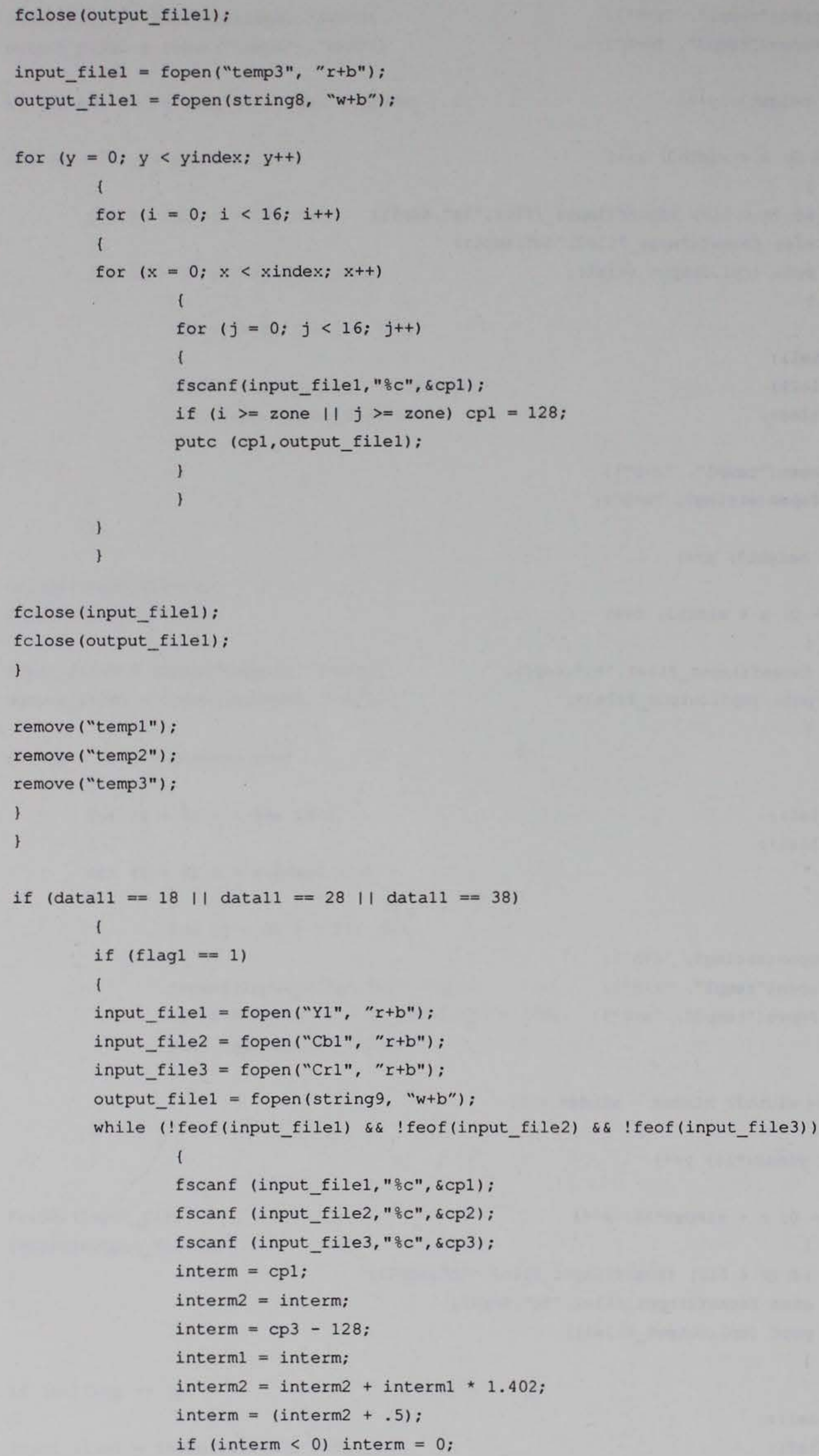




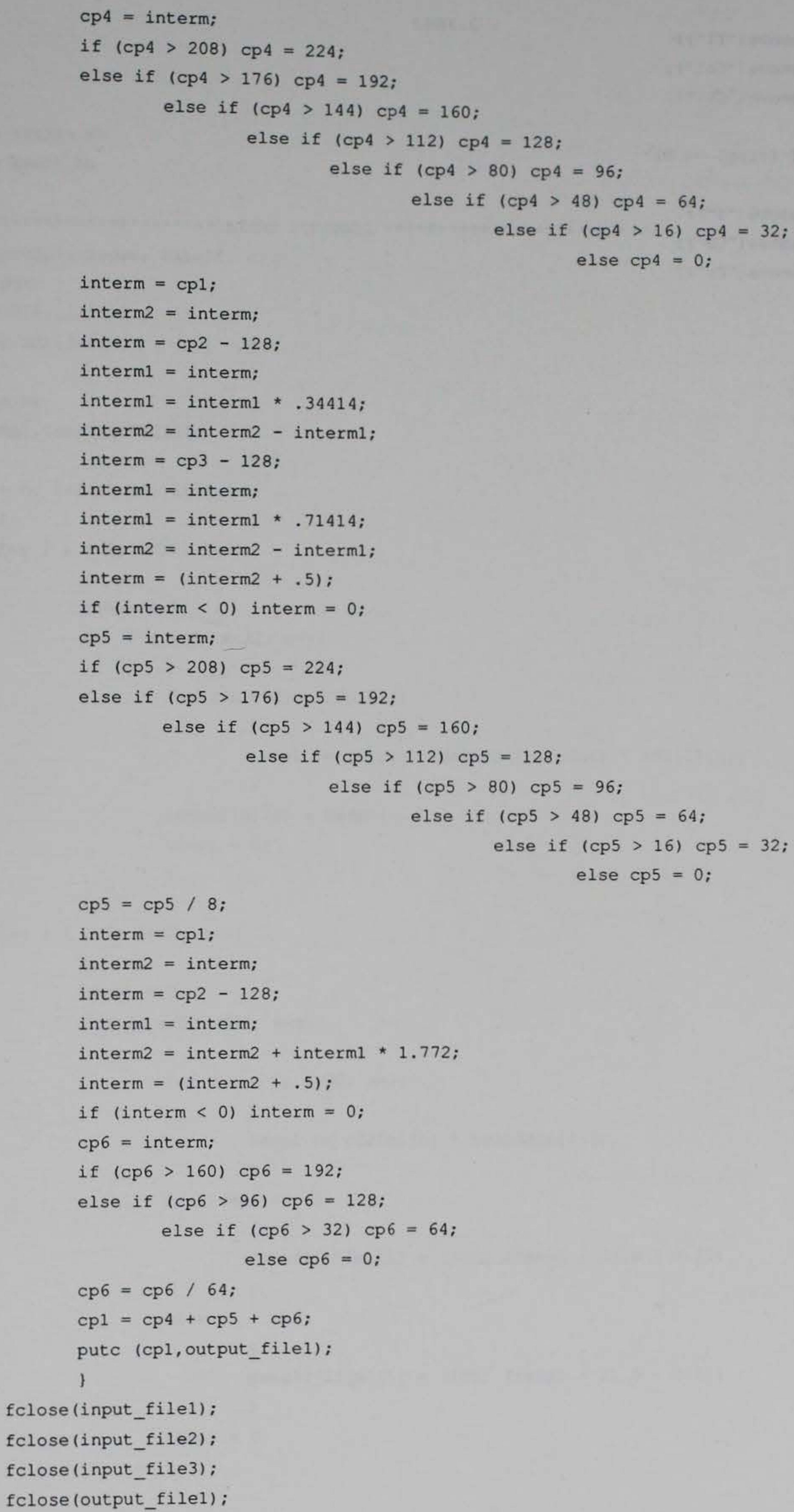




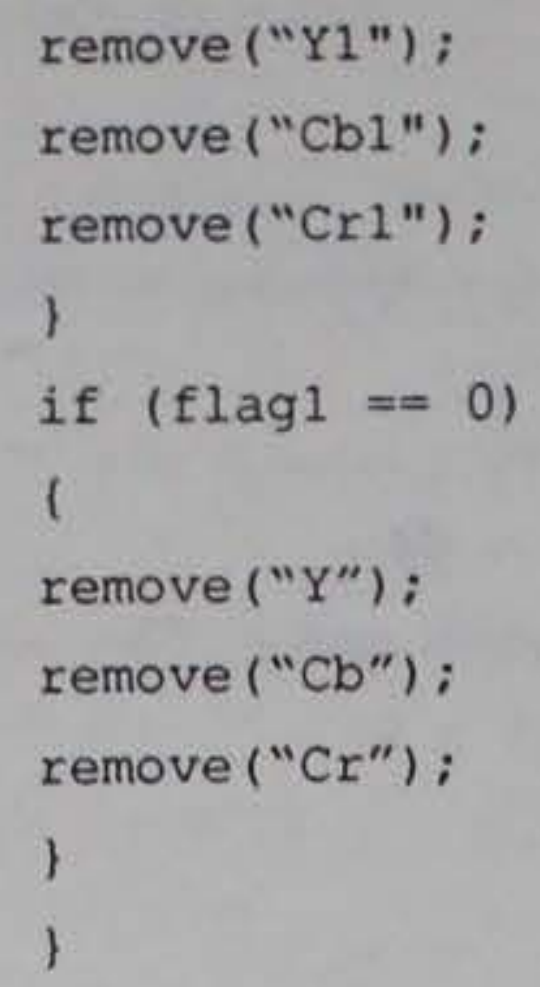

end1:

printf(""); 


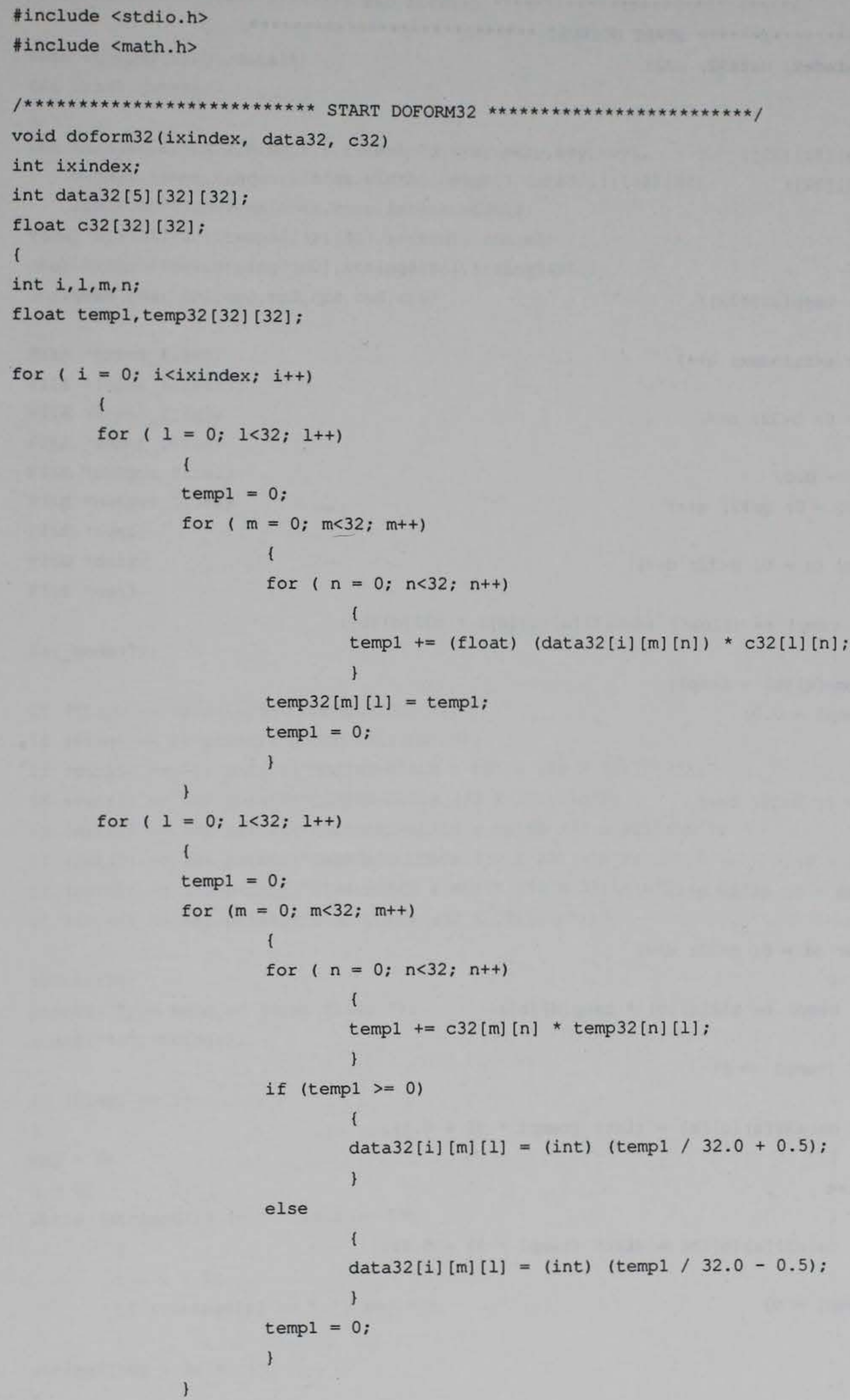




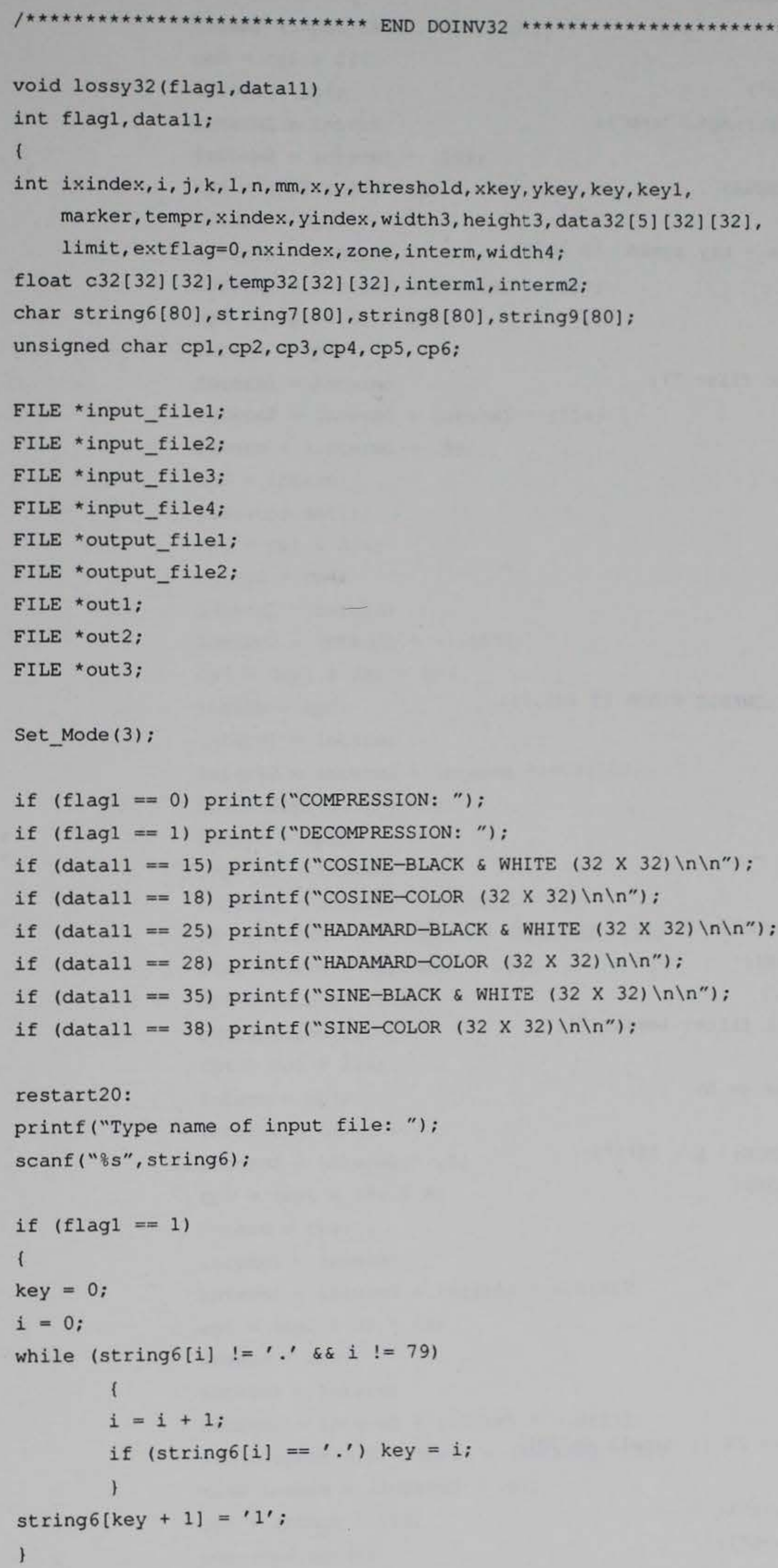




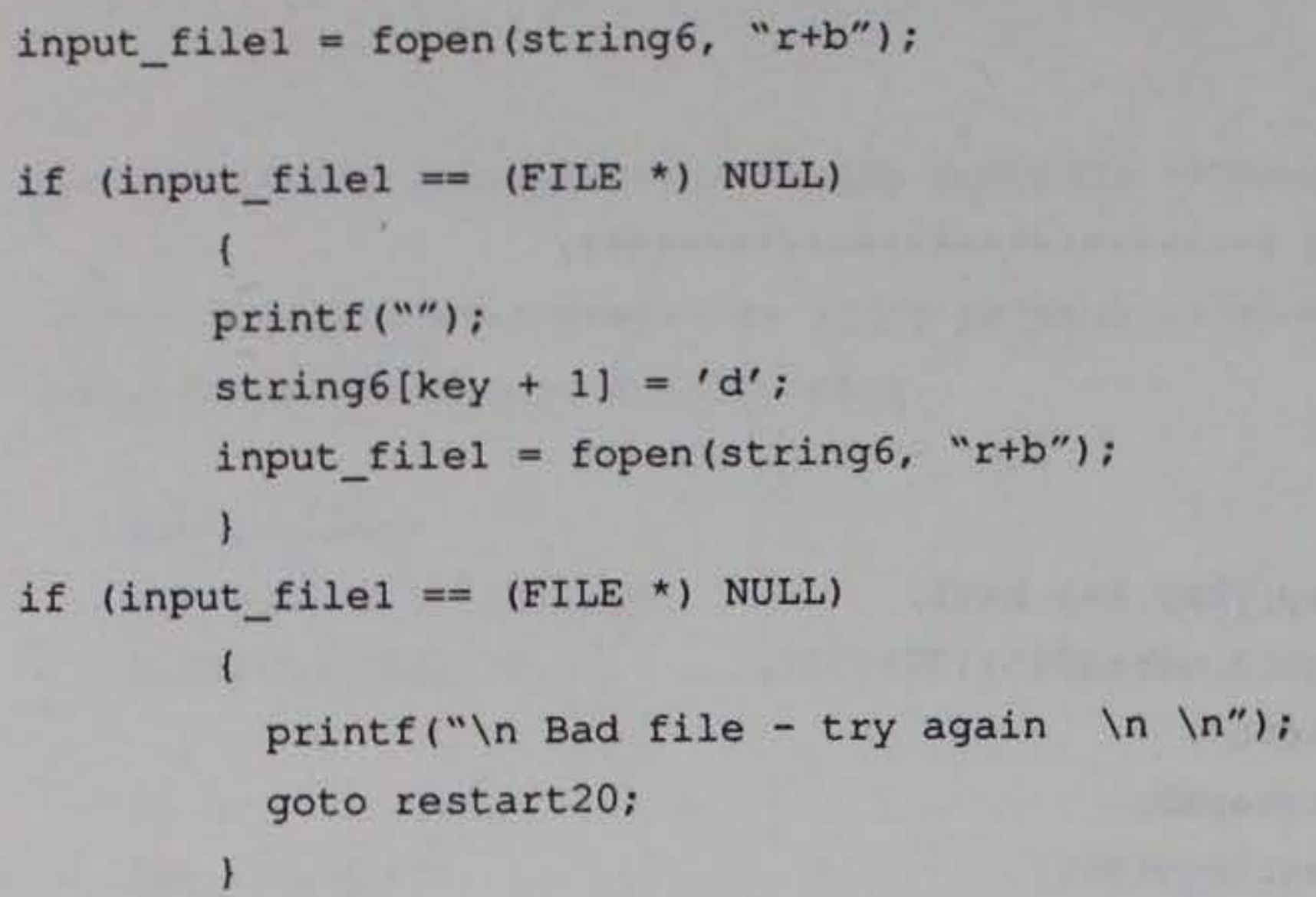

1 printf("\nMAXIMUM ALLOWABLE WIDTH IS 640."); 


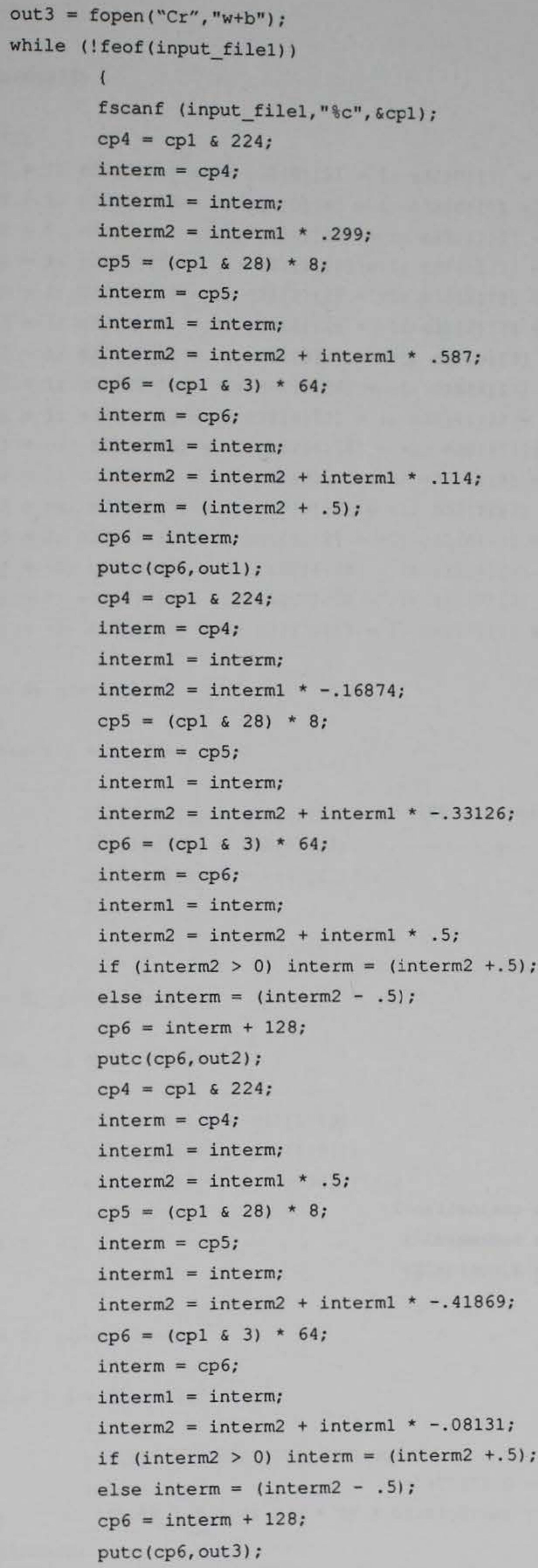


\}

fclose (input_file1) ;

fclose (out1);

fclose (out2);

fclose (out 3 ) ;

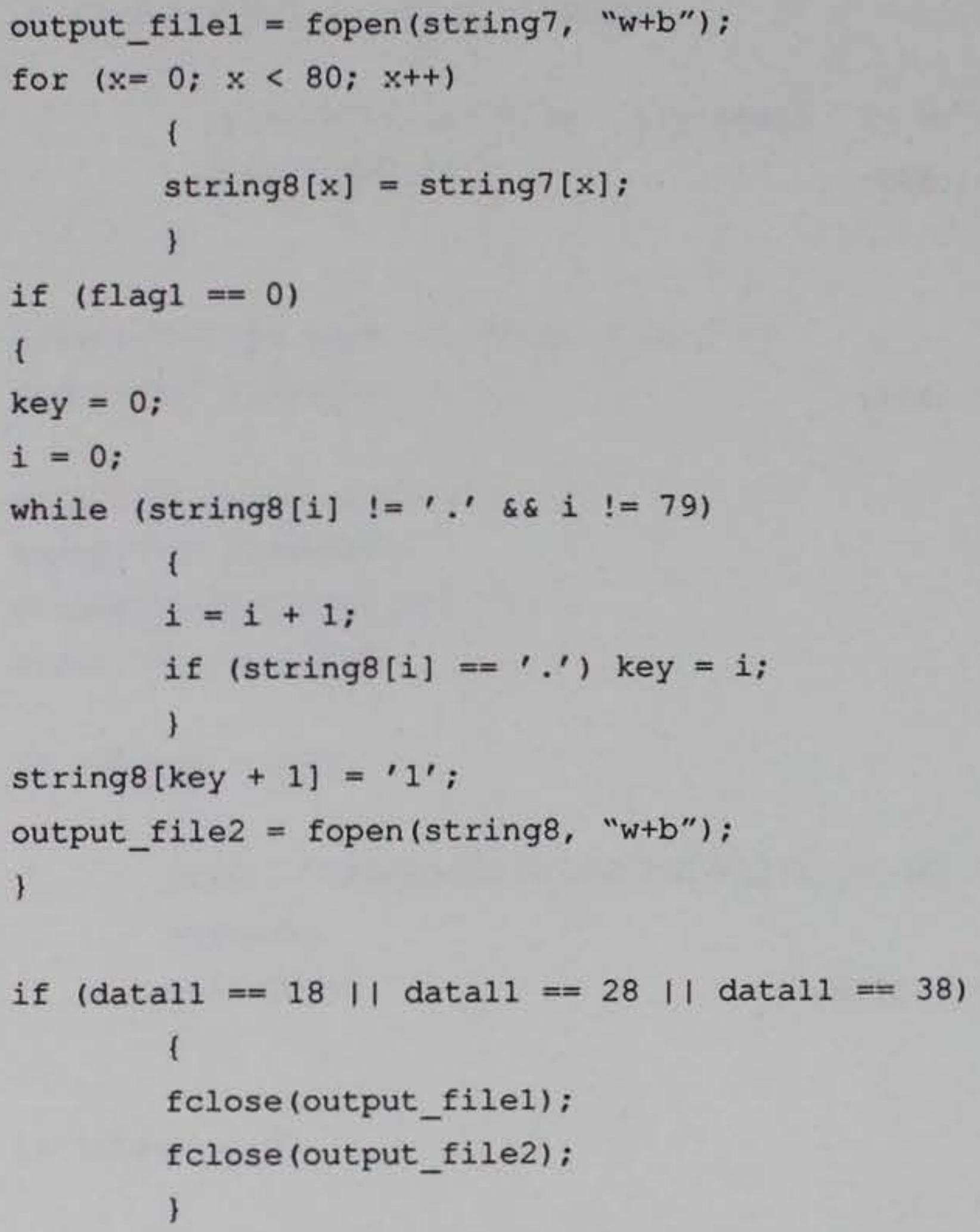


goto continue32;

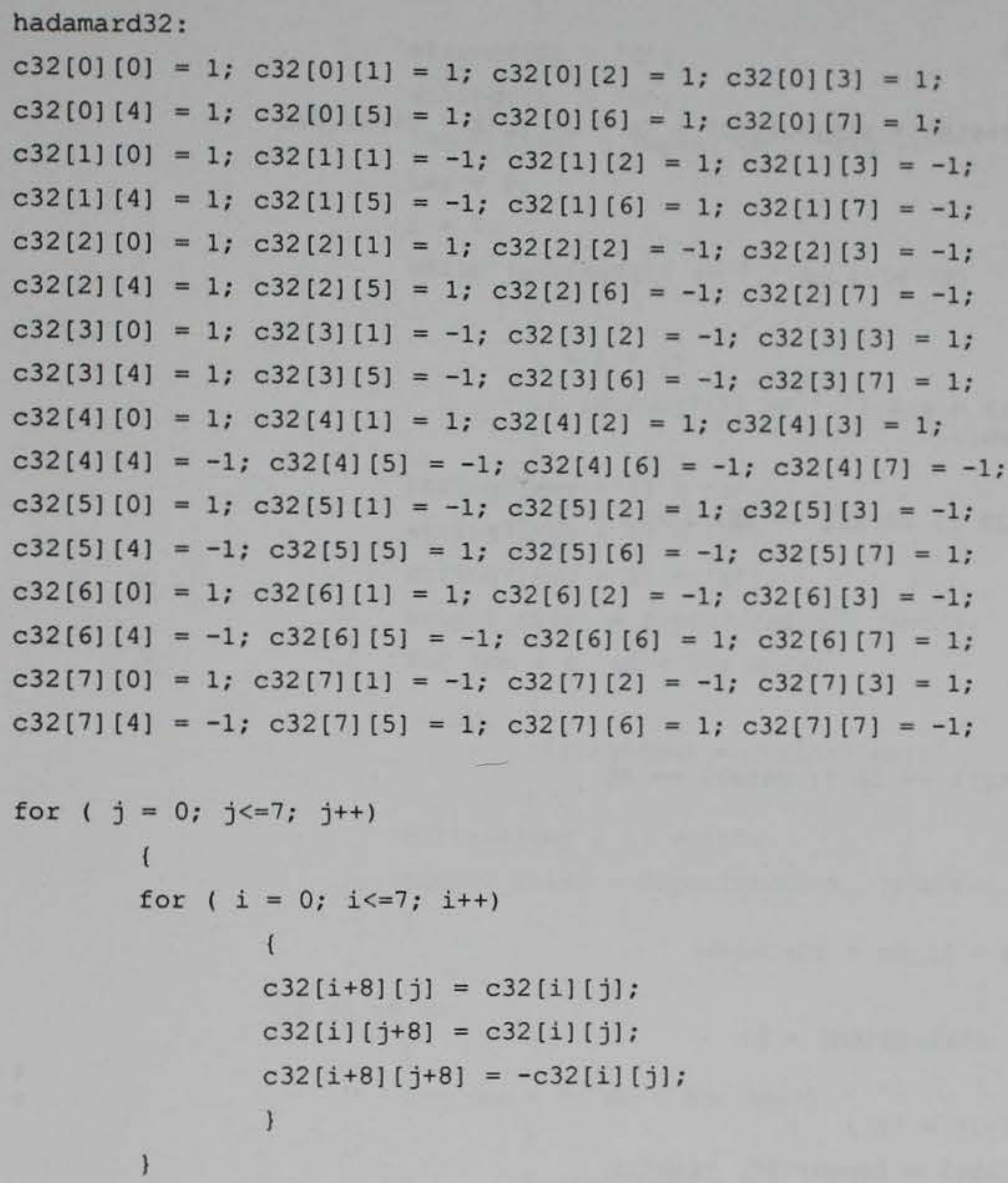

goto continue 32 ; 


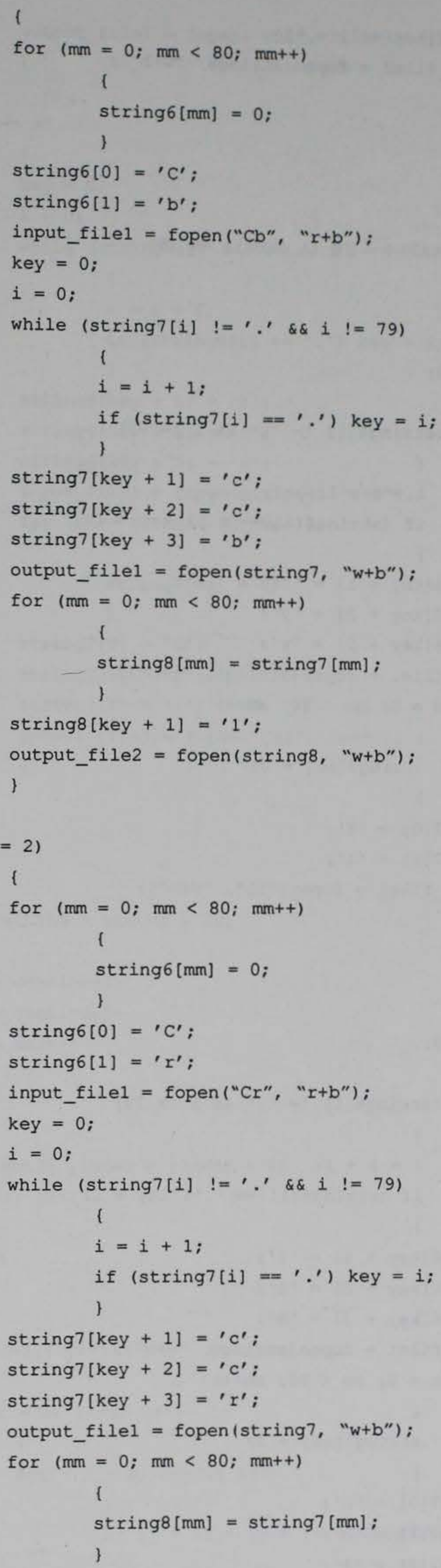




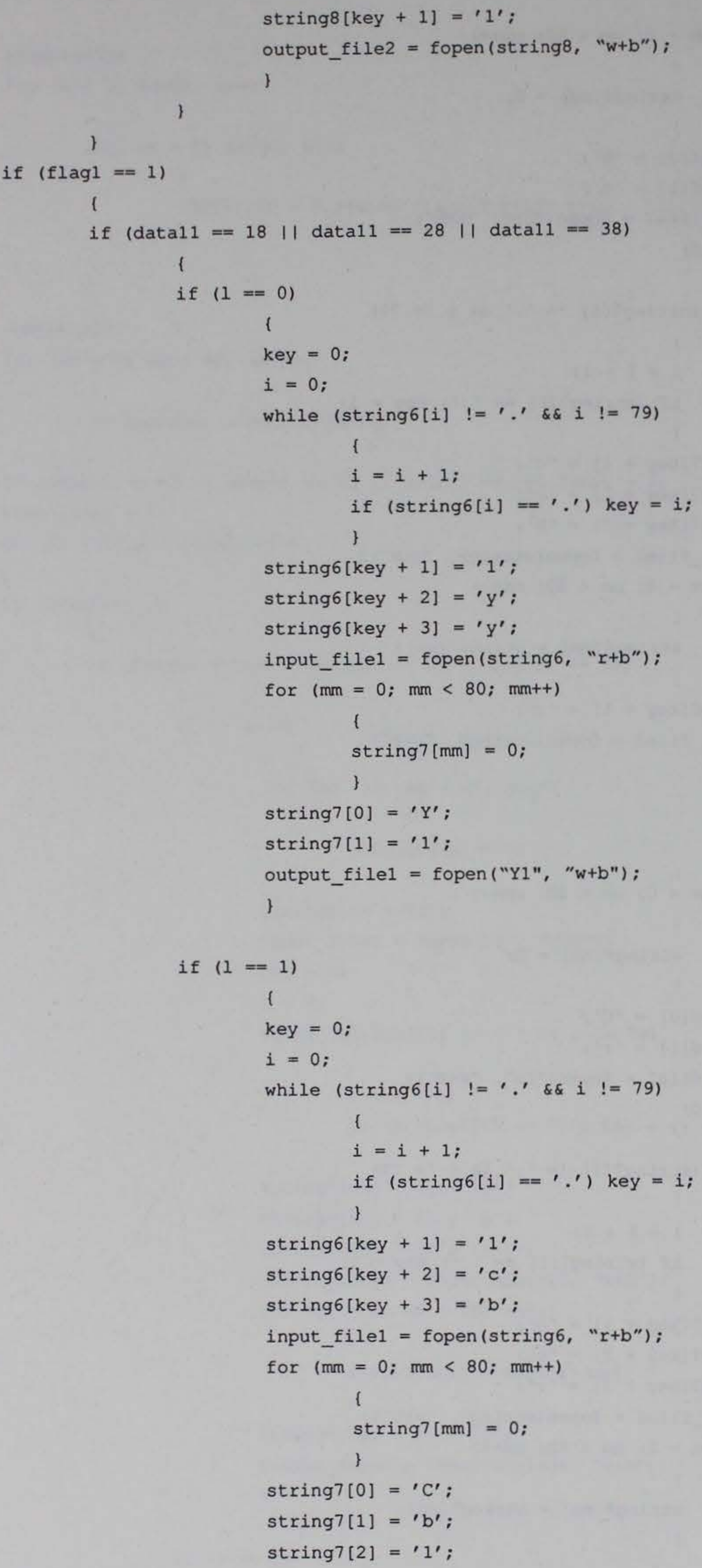


output_filel = fopen ("Cb1", "w+b") ;

)

if $(1==2)$

i

key $=0$;

$i=0$;

while (string6[i] != '.' \&\& $i \quad !=79$ )

1

$i=i+1 ;$

if (string $6[i]=={ }^{\prime} \cdot$ ) key $=i$;

\}

string6 $[$ key +1$]=$ ' 1 ';

string $6[k e y+2]=' c^{\prime}$;

string $6[$ key +3$]=$ ' $r$ ';

input_filel $=$ fopen (string6, " $r+b ")$;

for $(\mathrm{mm}=0 ; \mathrm{mm}<80 ; \mathrm{mm}++)$

\{

string $7[\mathrm{~mm}]=0$;

I

string $7[0]=' C^{\prime}$;

string $7[1]=' r^{\prime}$;

string 72$]=$ ' 1 ';

output_filel = fopen ("Crl", "w+b");

\}

)

\}

width $4=32{ }^{\star} x$ index;

if (width $4=$ width 3 ) width $4=$ width $4+32$;

if (extflag $==2$ ) goto continue2;

if (extflag $==1$ ) goto continuel;

if (extflag $==0$ ) goto next;

continue 3 :

printf ("In.");

if $(32$ * $y$ index $!=$ height 3$)$ yindex $=y$ index +1 ;

for $(j=0 ; j<=y i n d e x-1 ; j++)$

1

if $(f \operatorname{lag} 1=0)$

1

printf(".");

for $(y=0 ; y<=31 ; y++)$

1

for $(i=0 ; i<=4 ; i++)$

1

for $(\mathrm{x}=0 ; \mathrm{x}<=31 ; \mathrm{x}++)$

i

if $(j * 32+(y+1)>$ height 3$)$ 


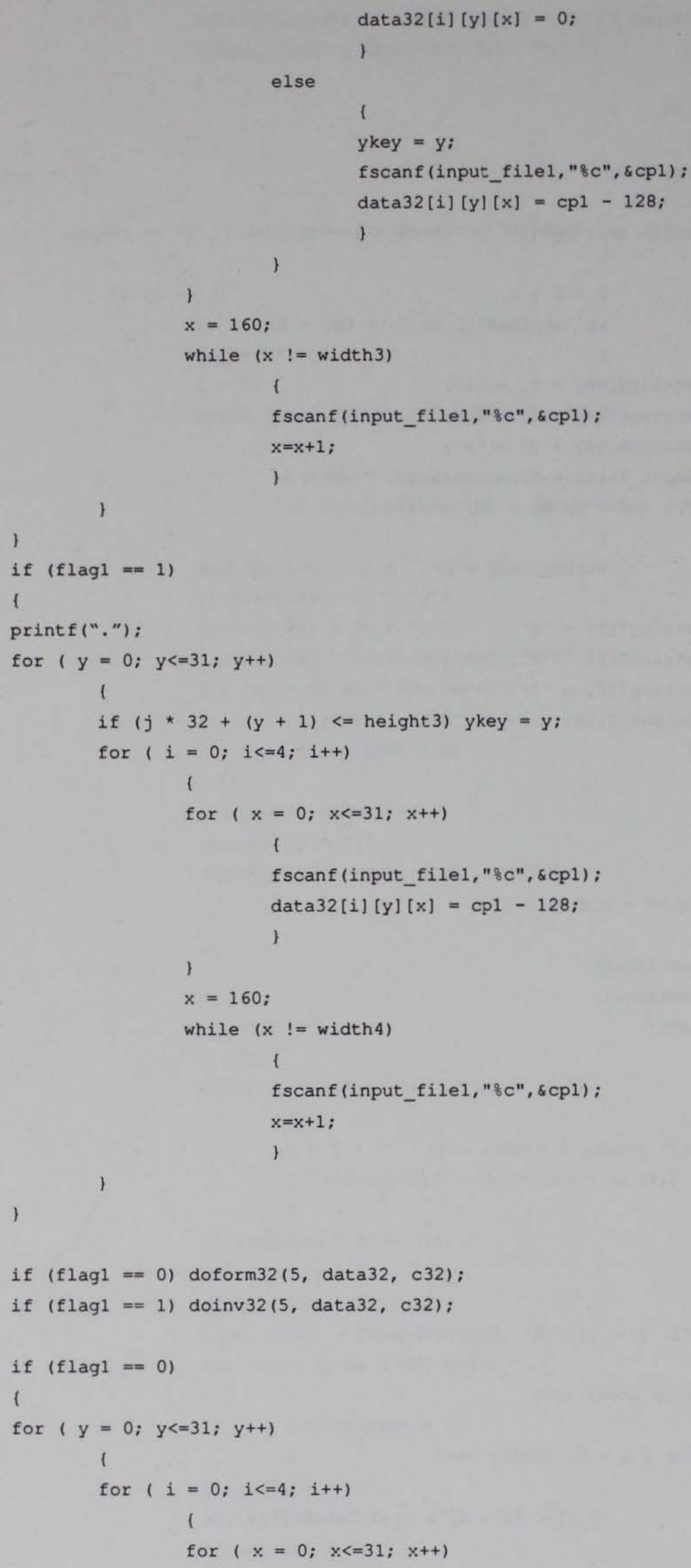




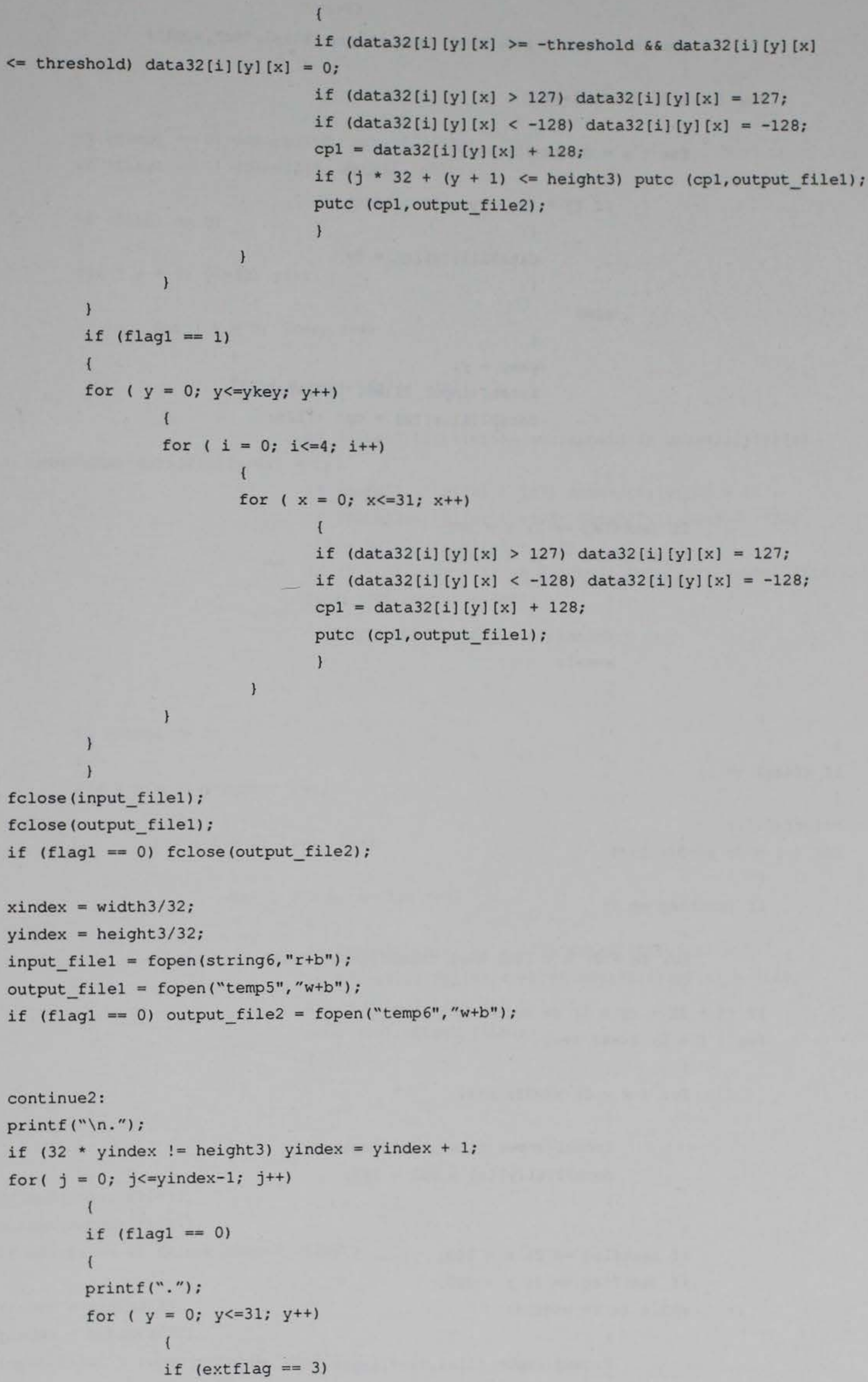




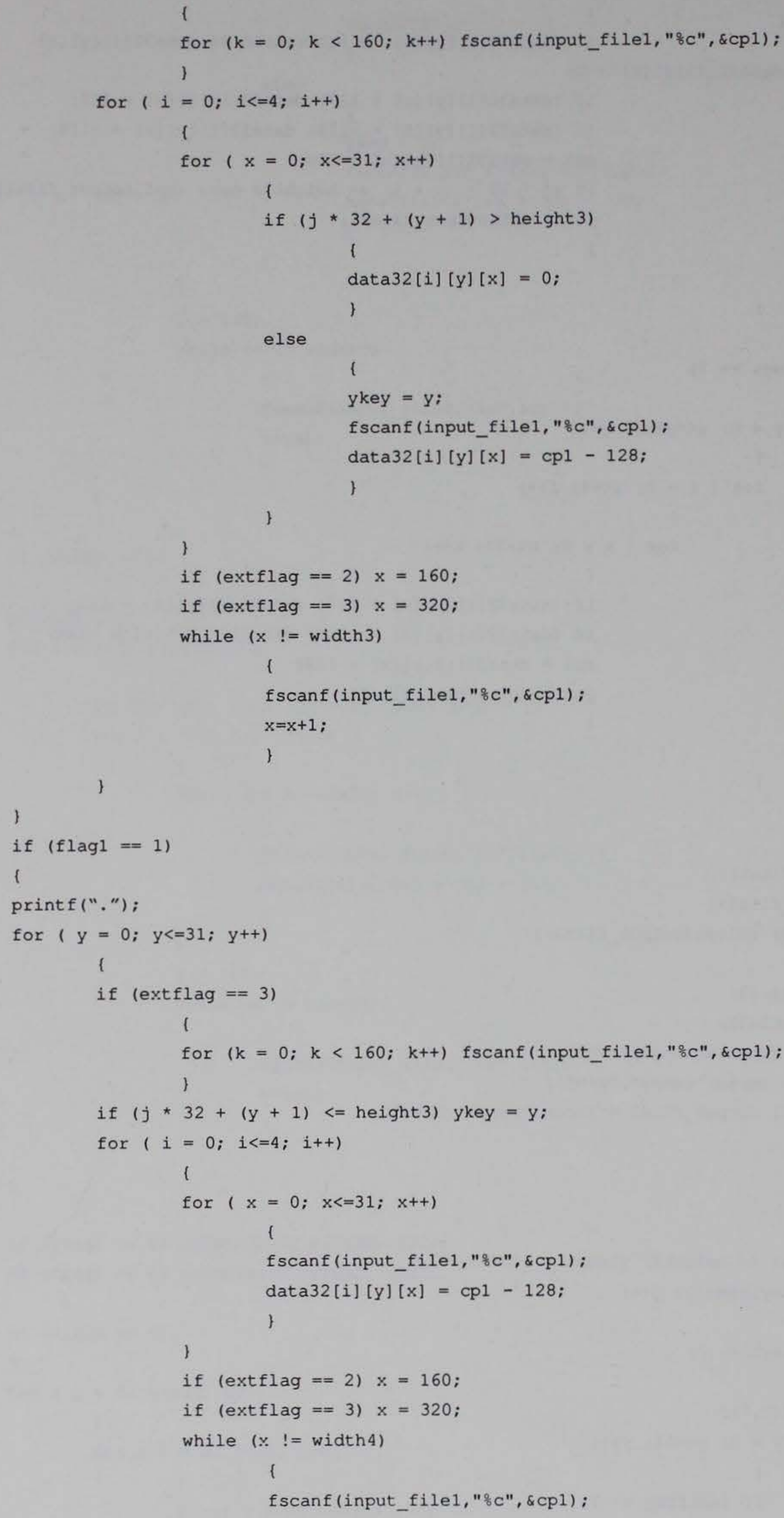




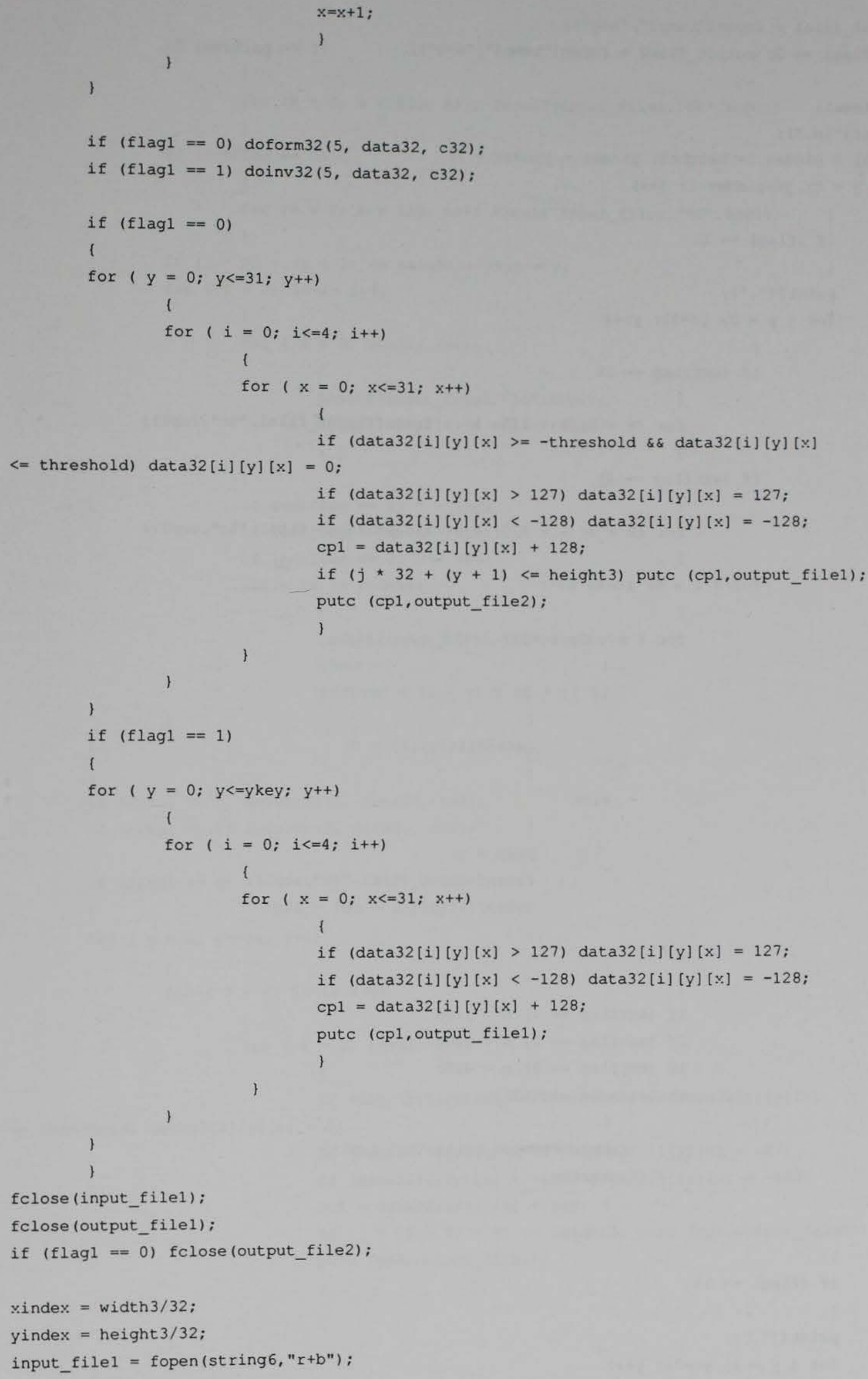




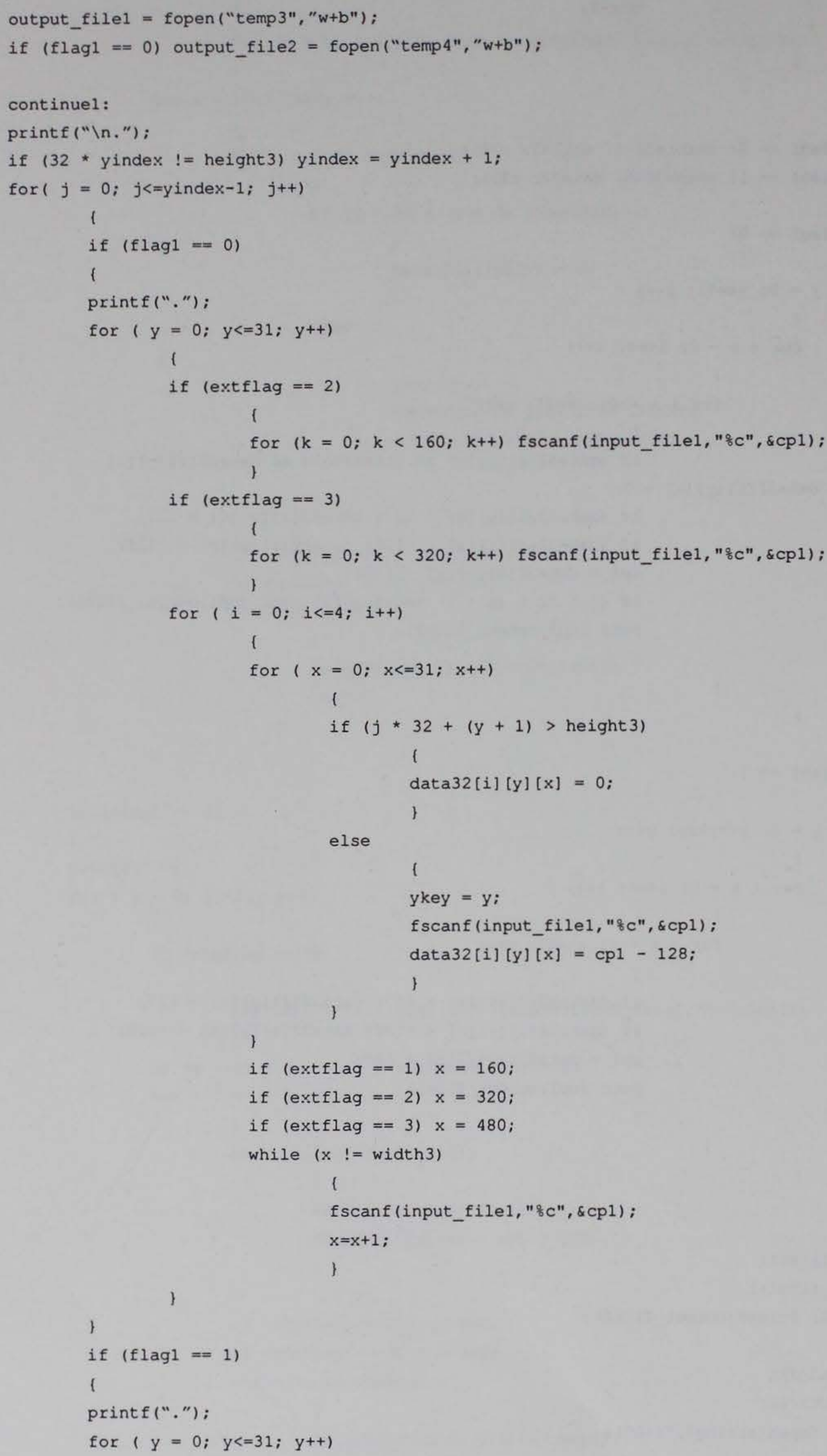




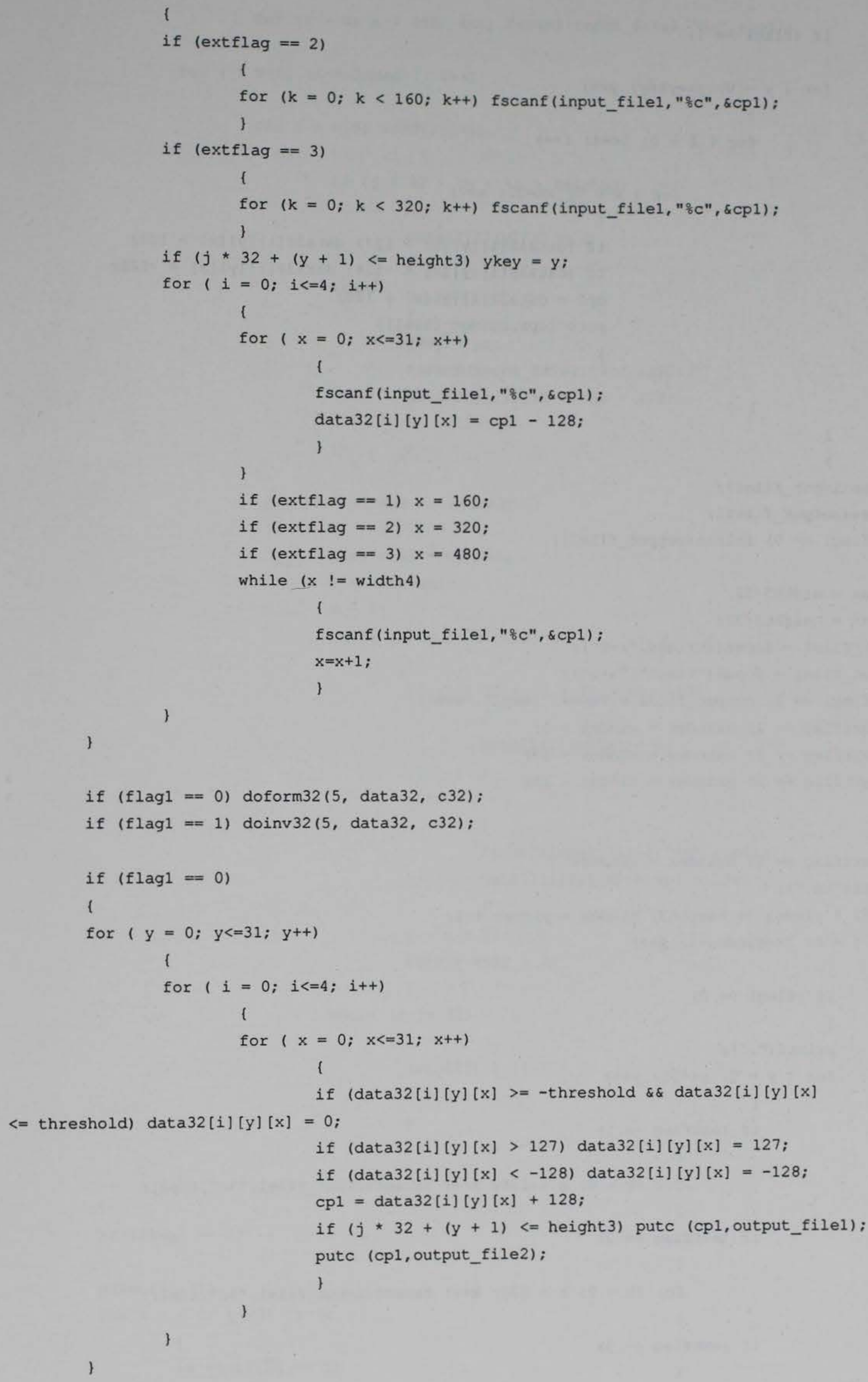




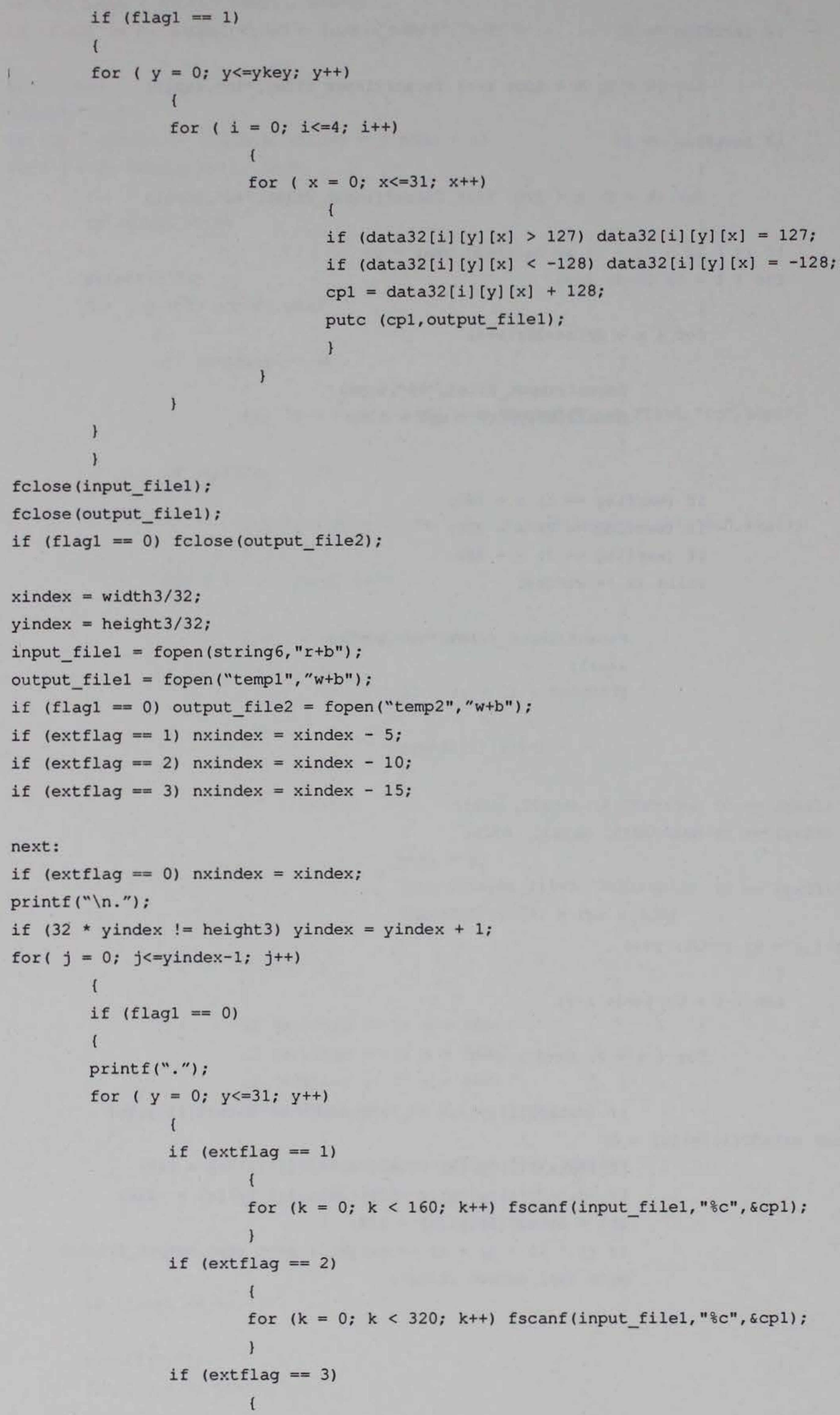




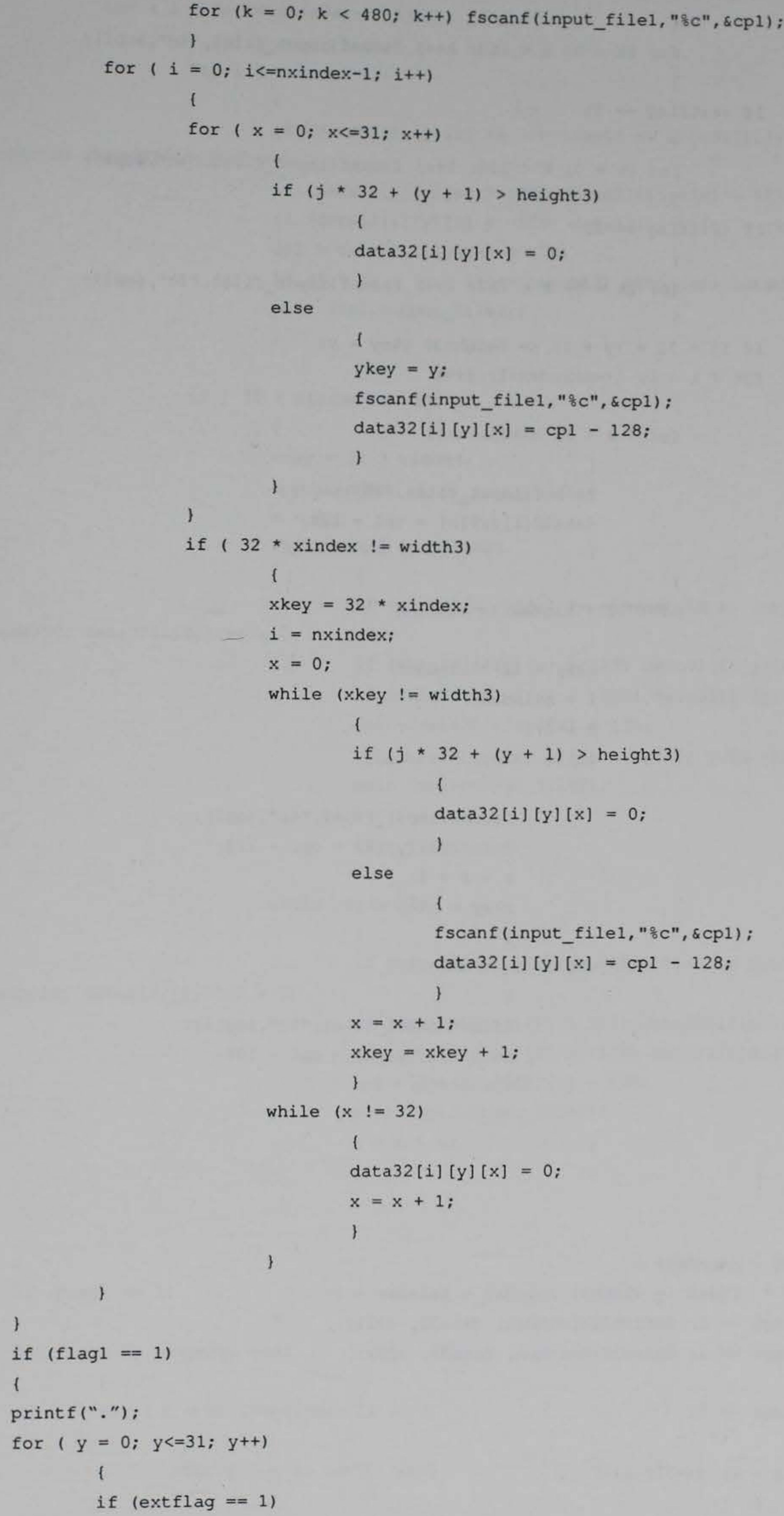




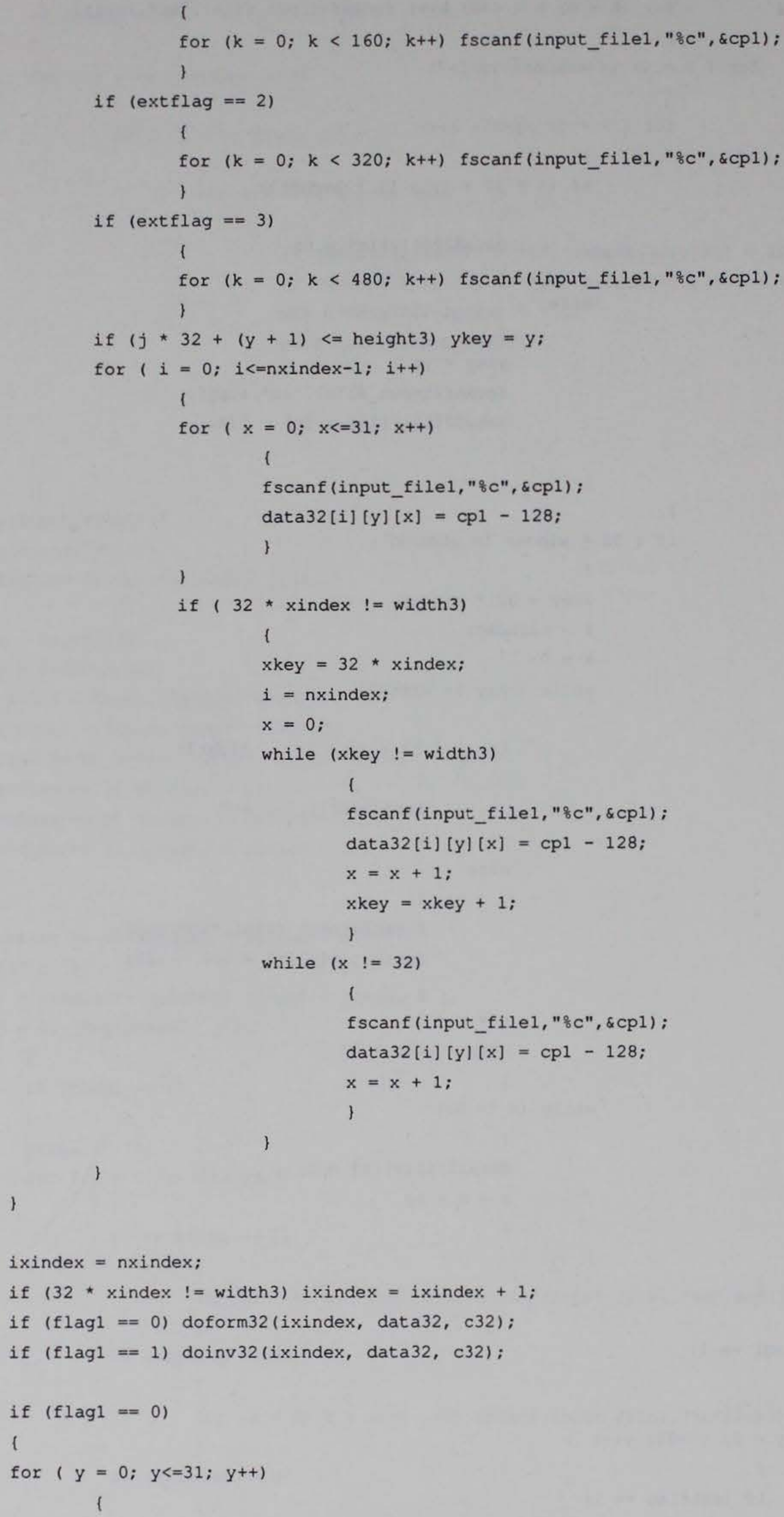




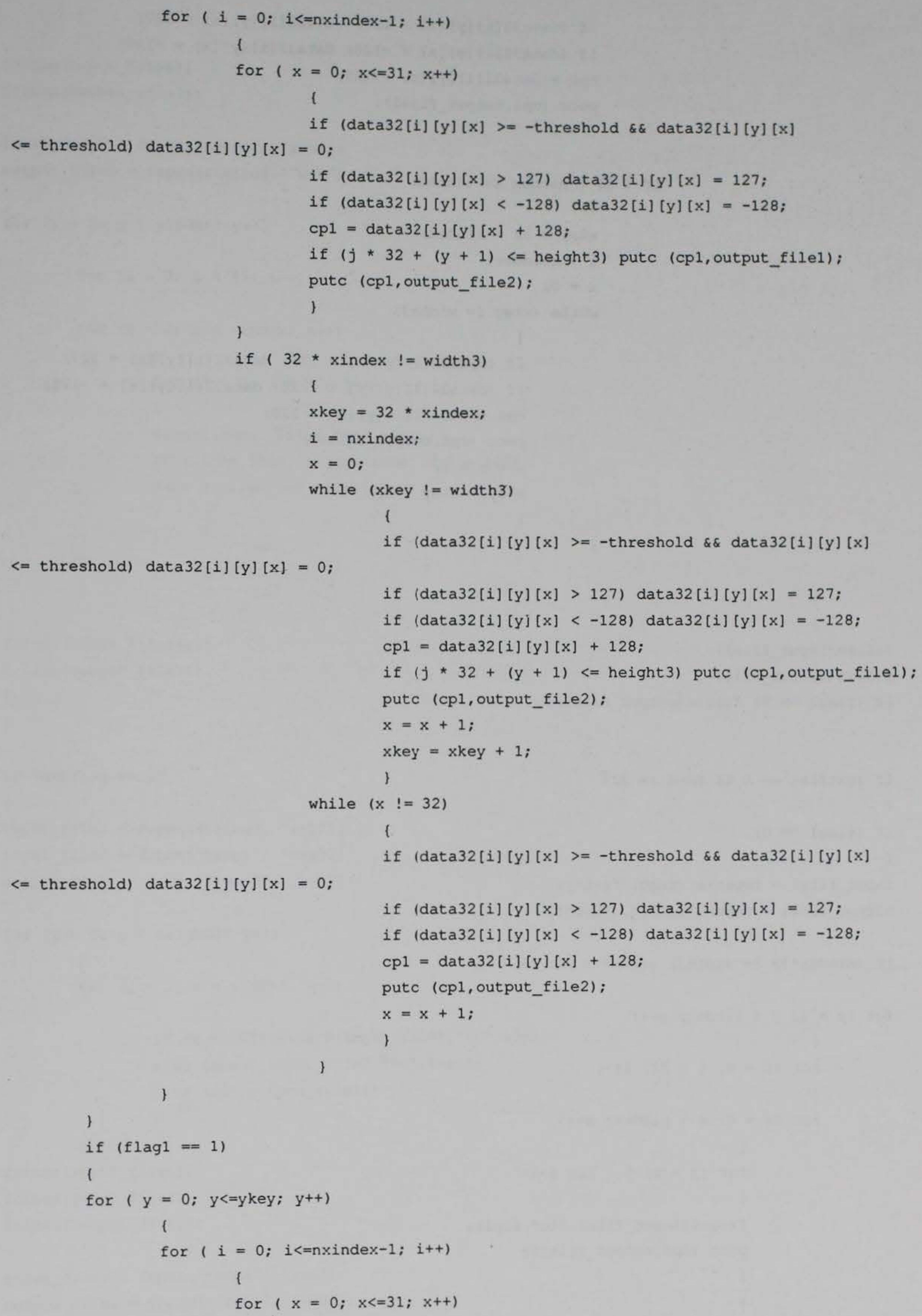




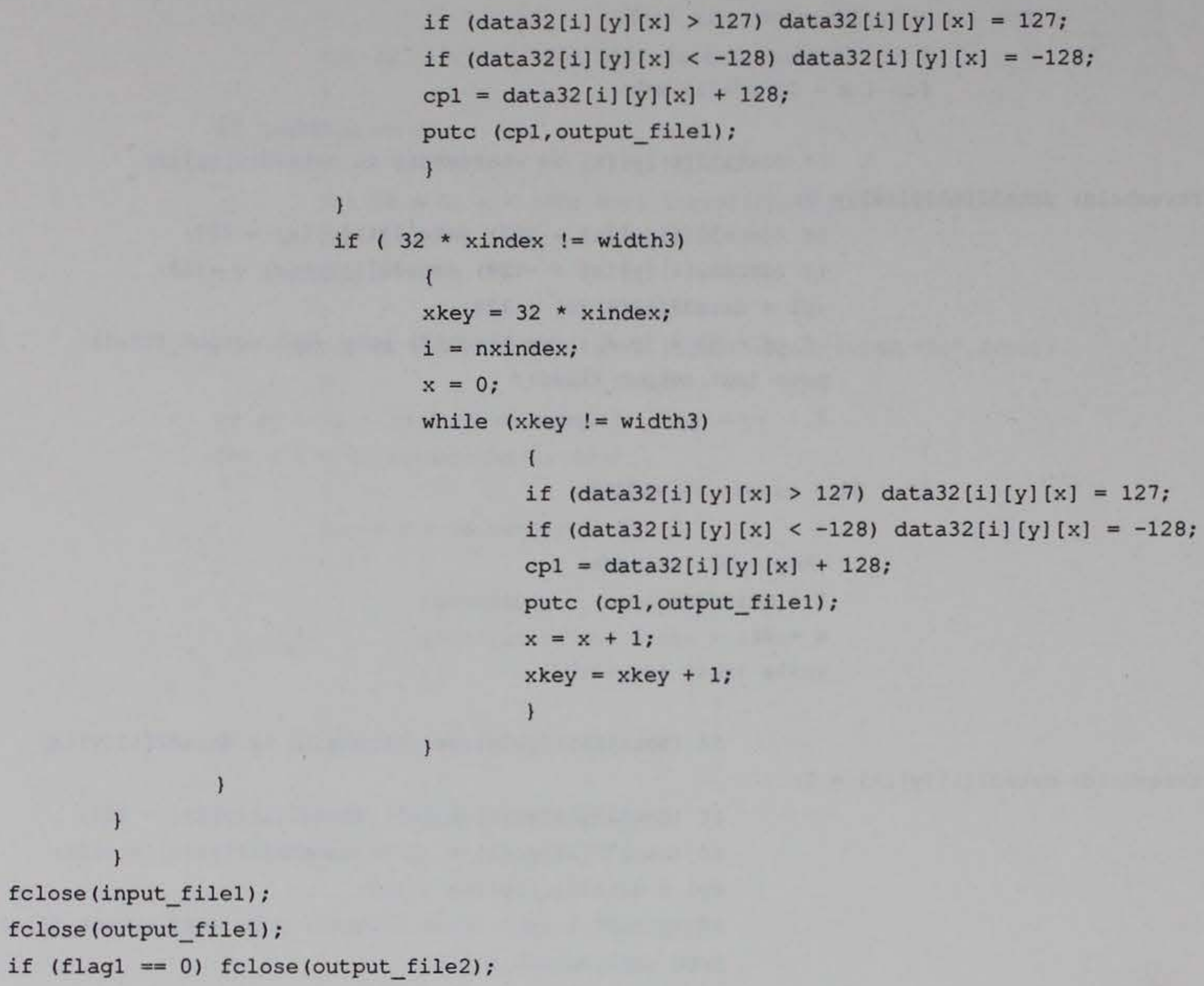




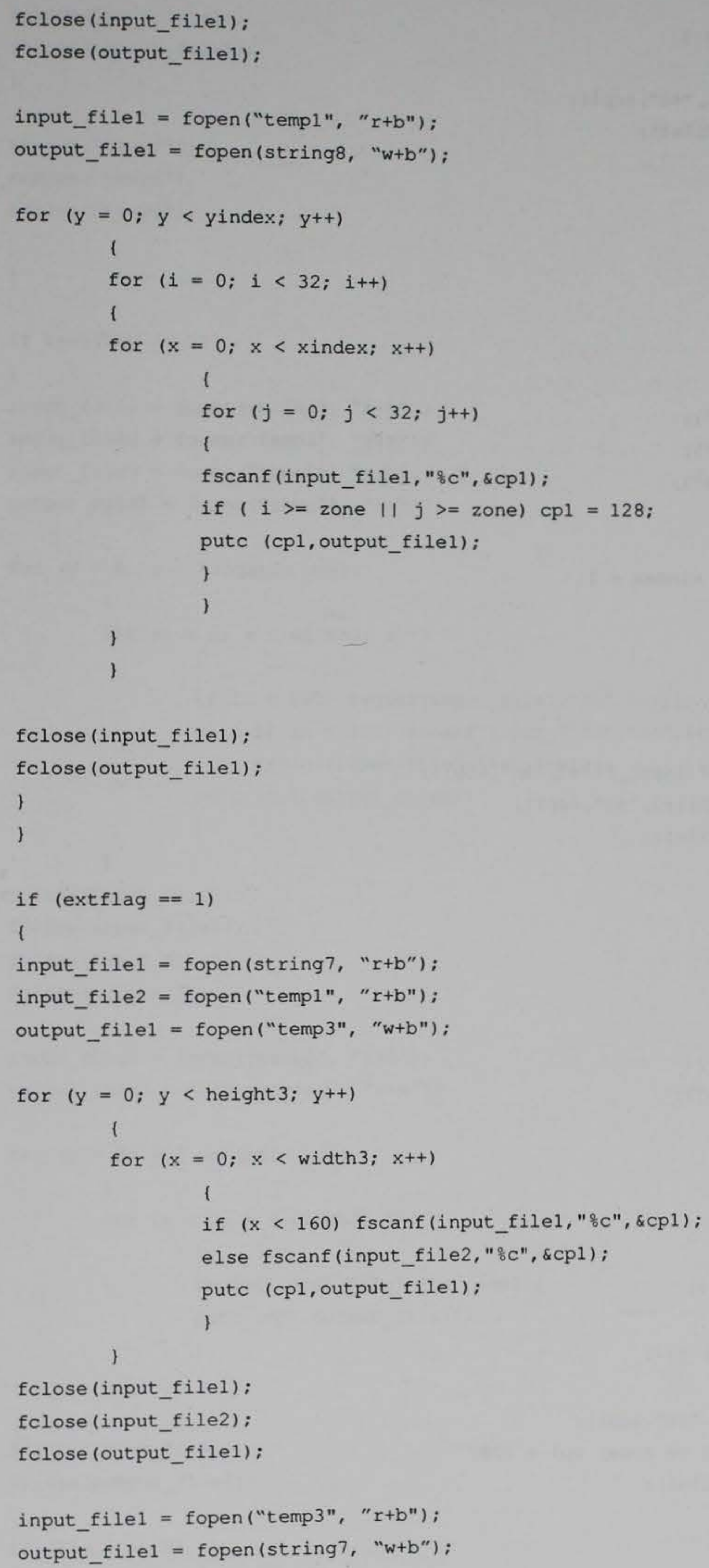




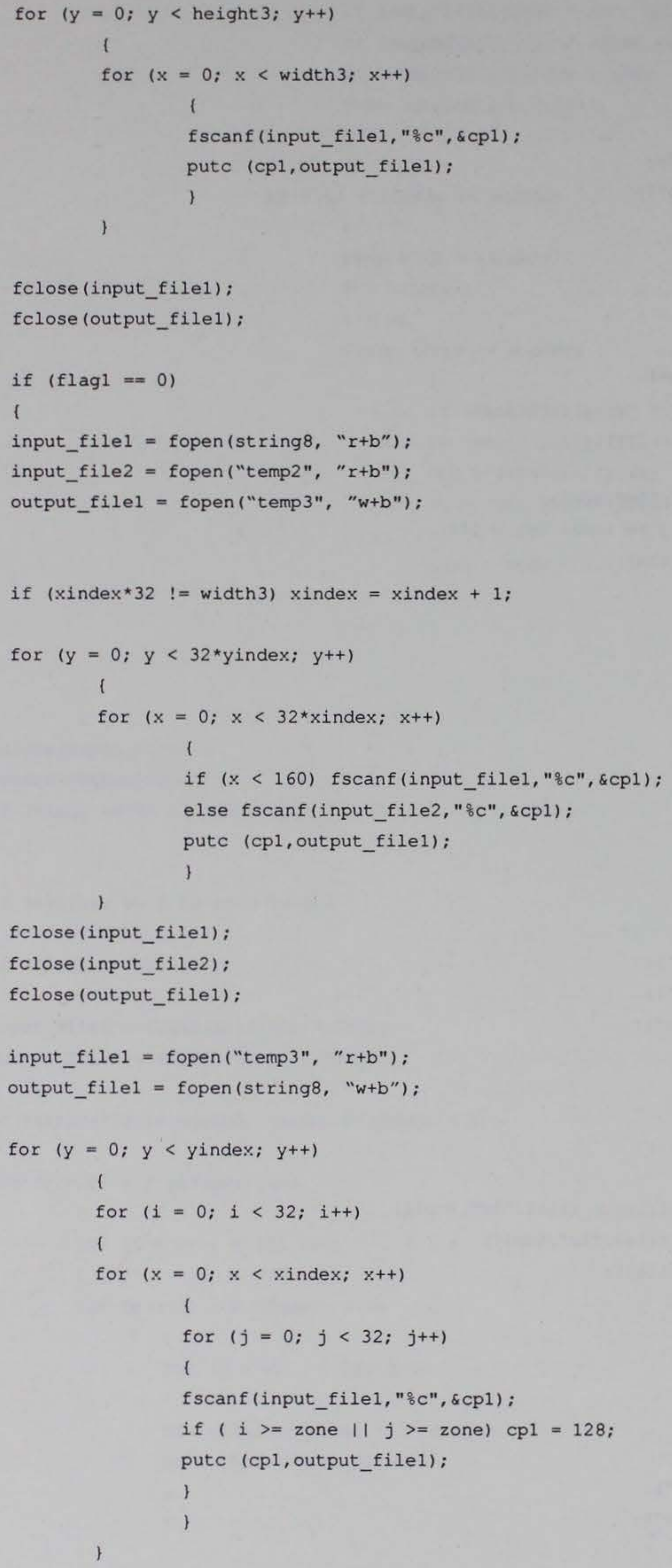




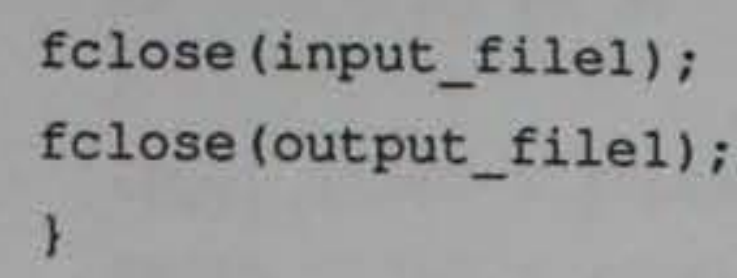




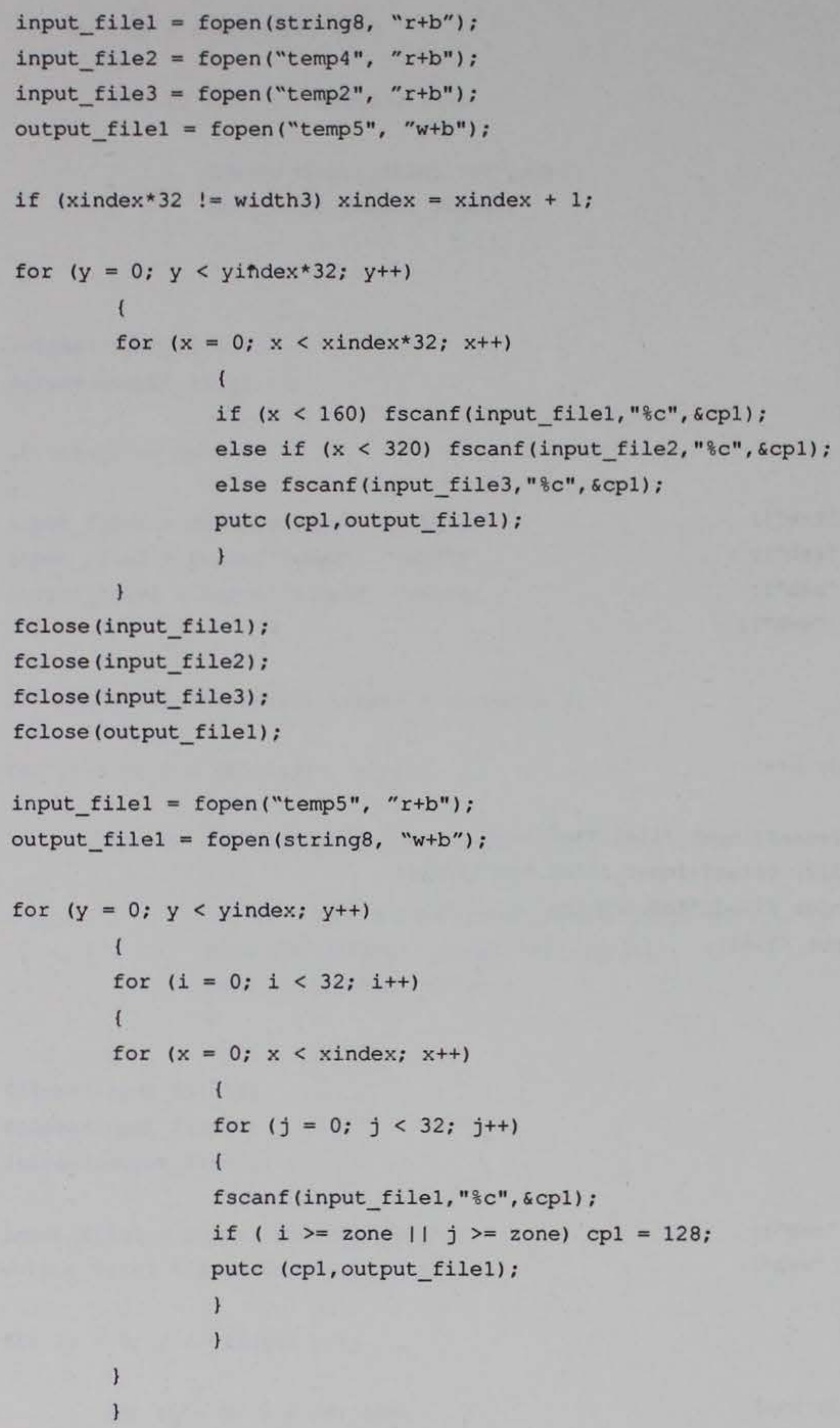




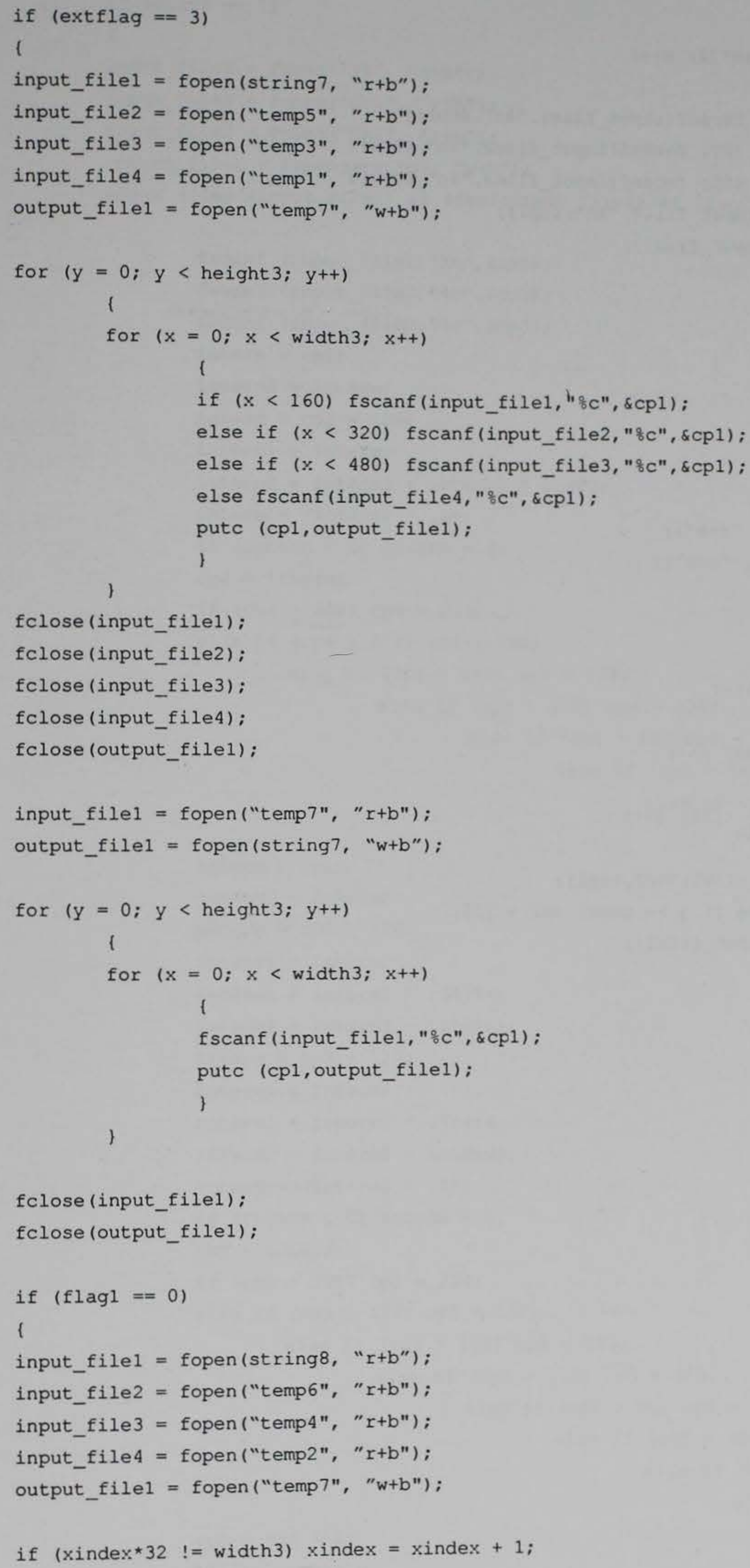




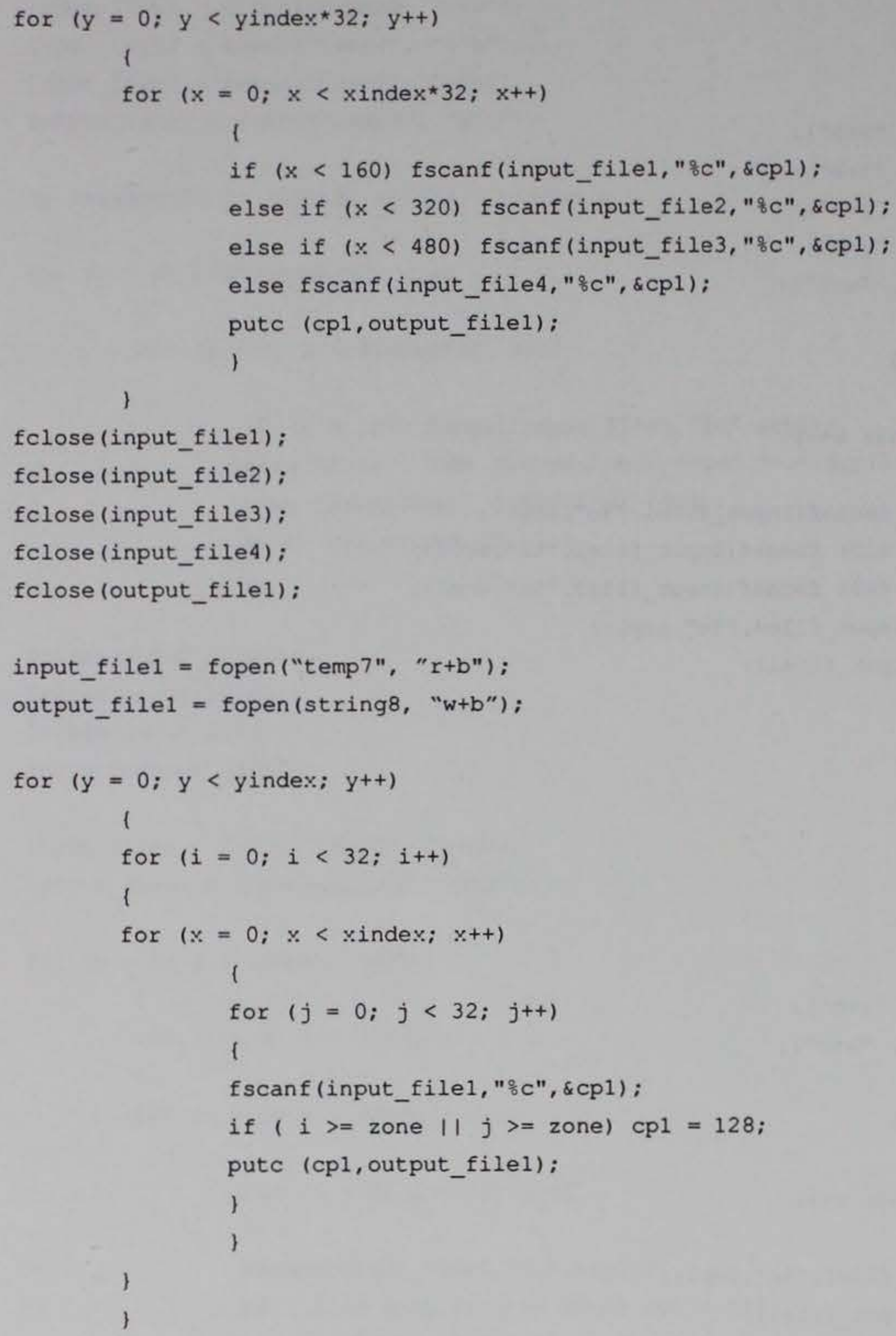




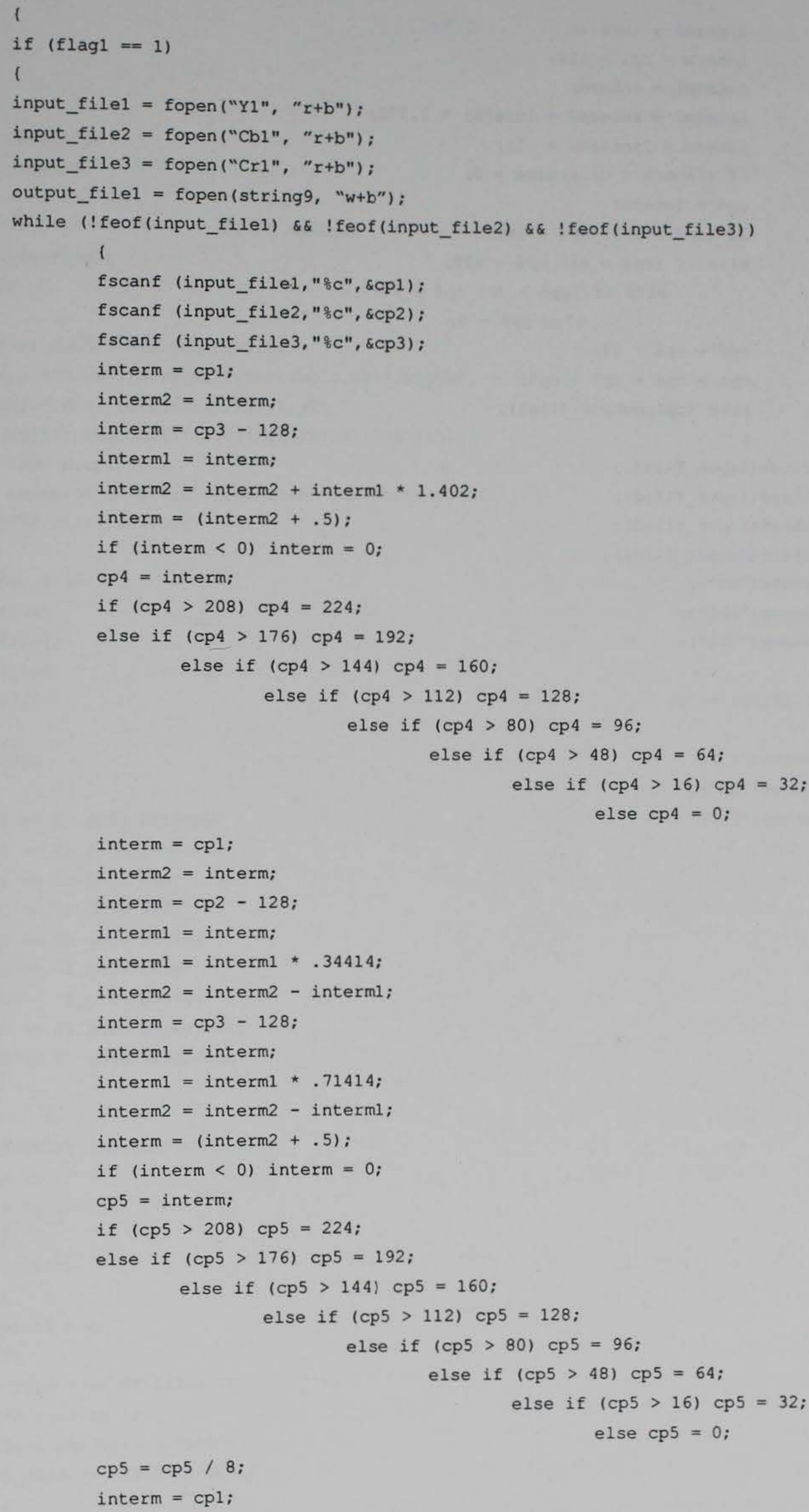




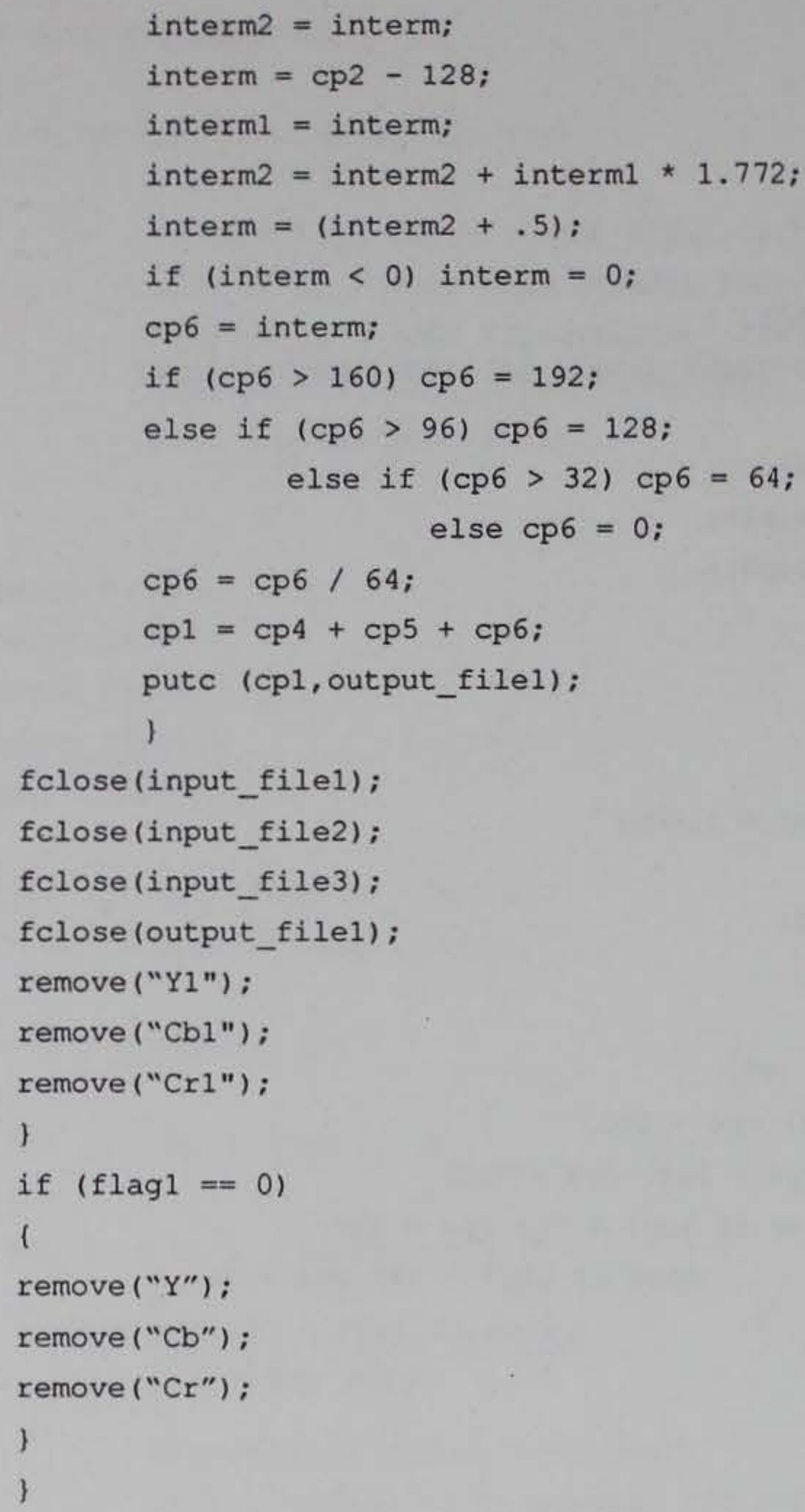




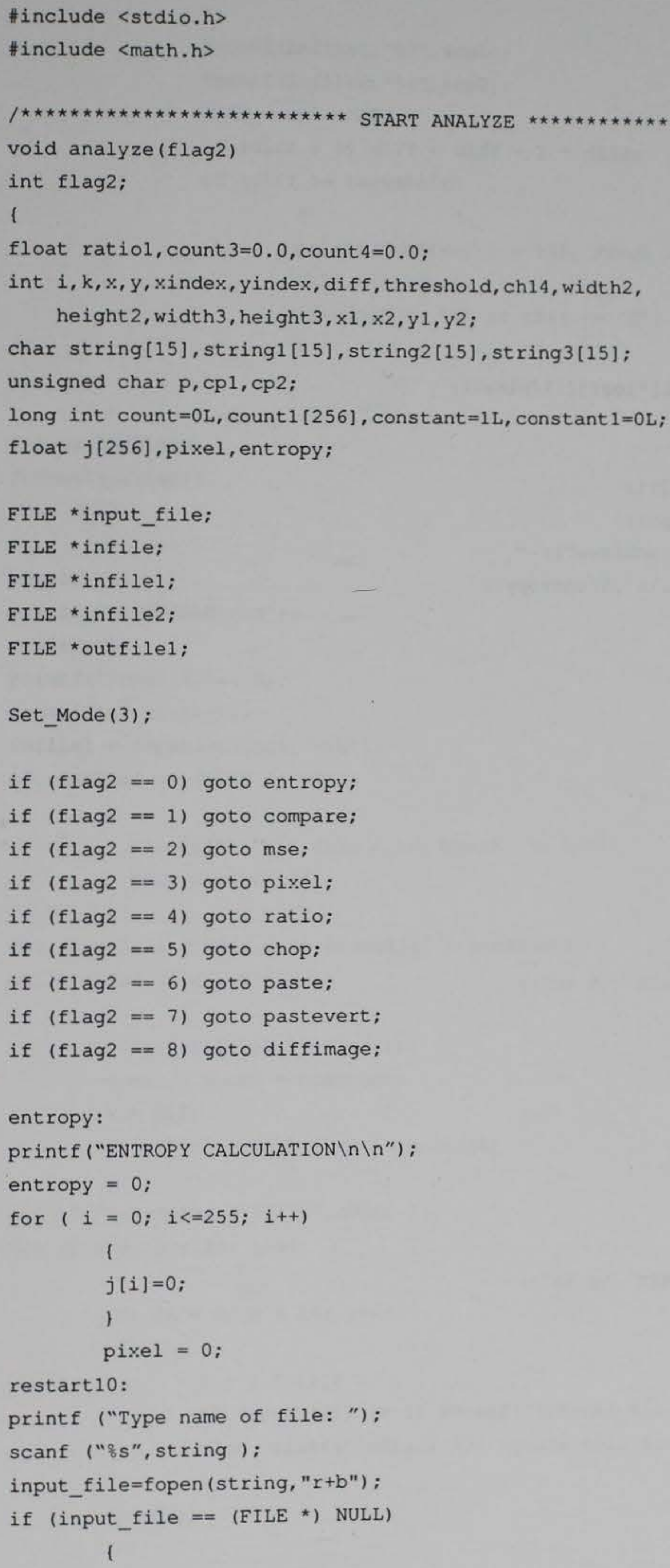




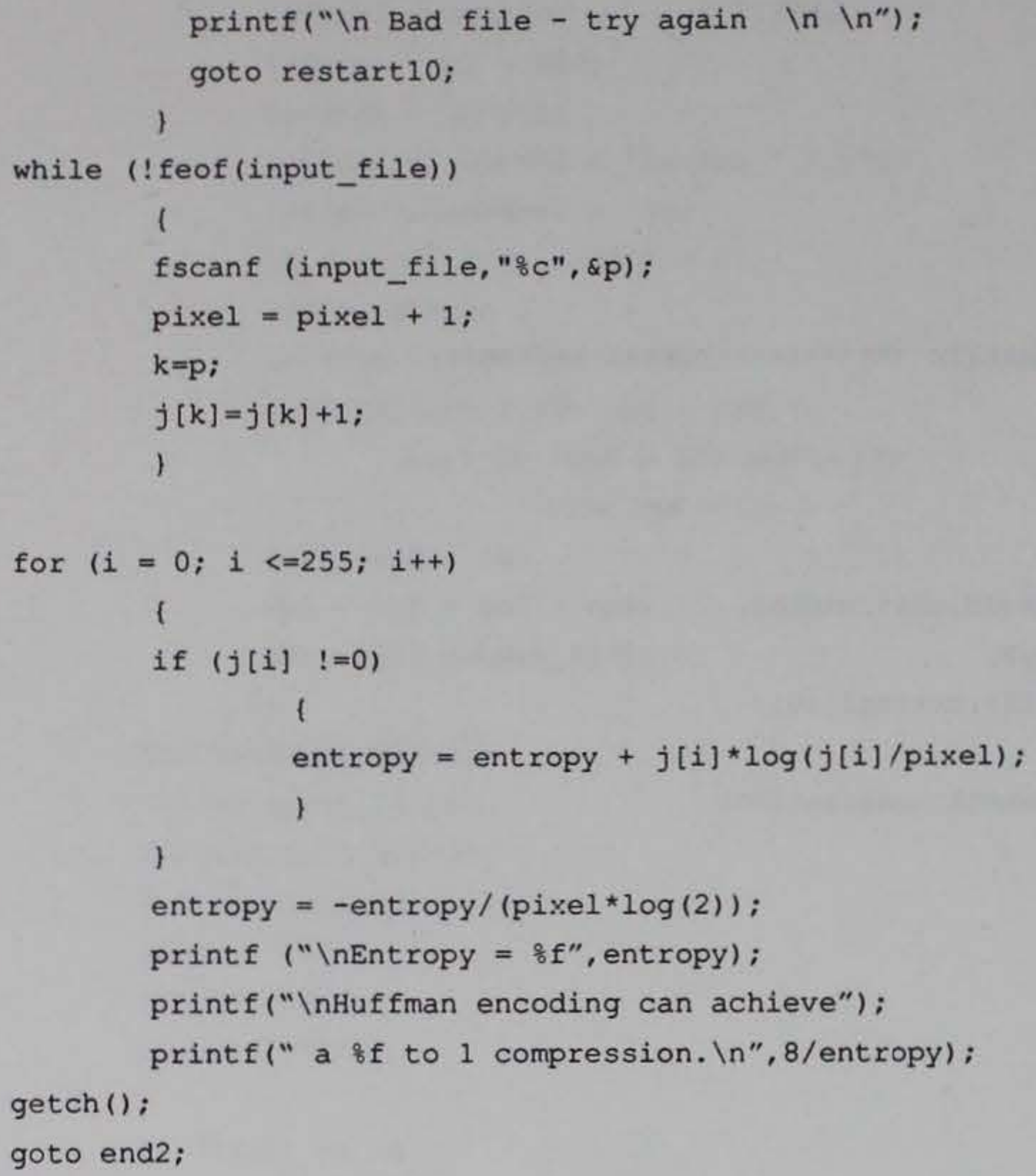




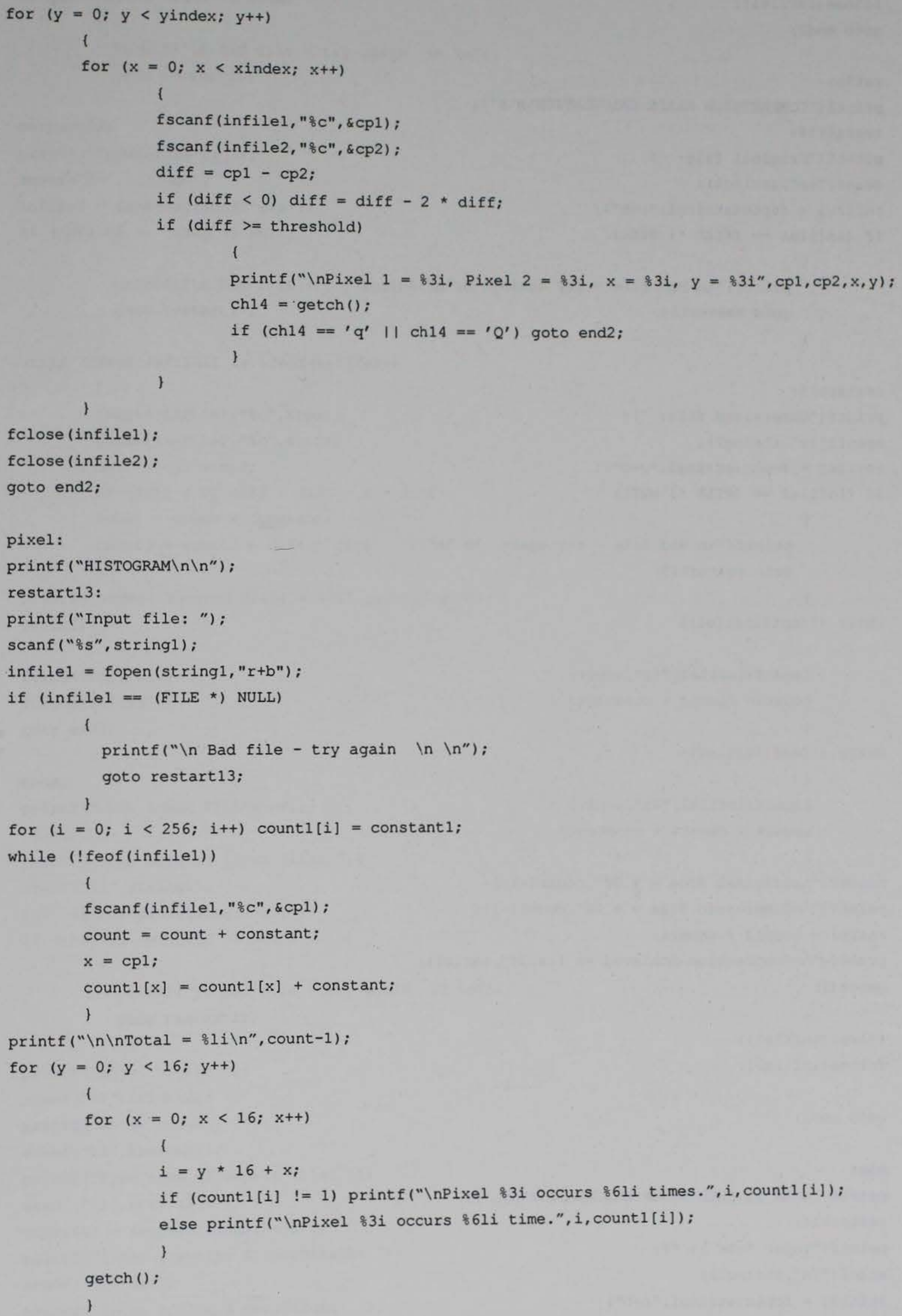




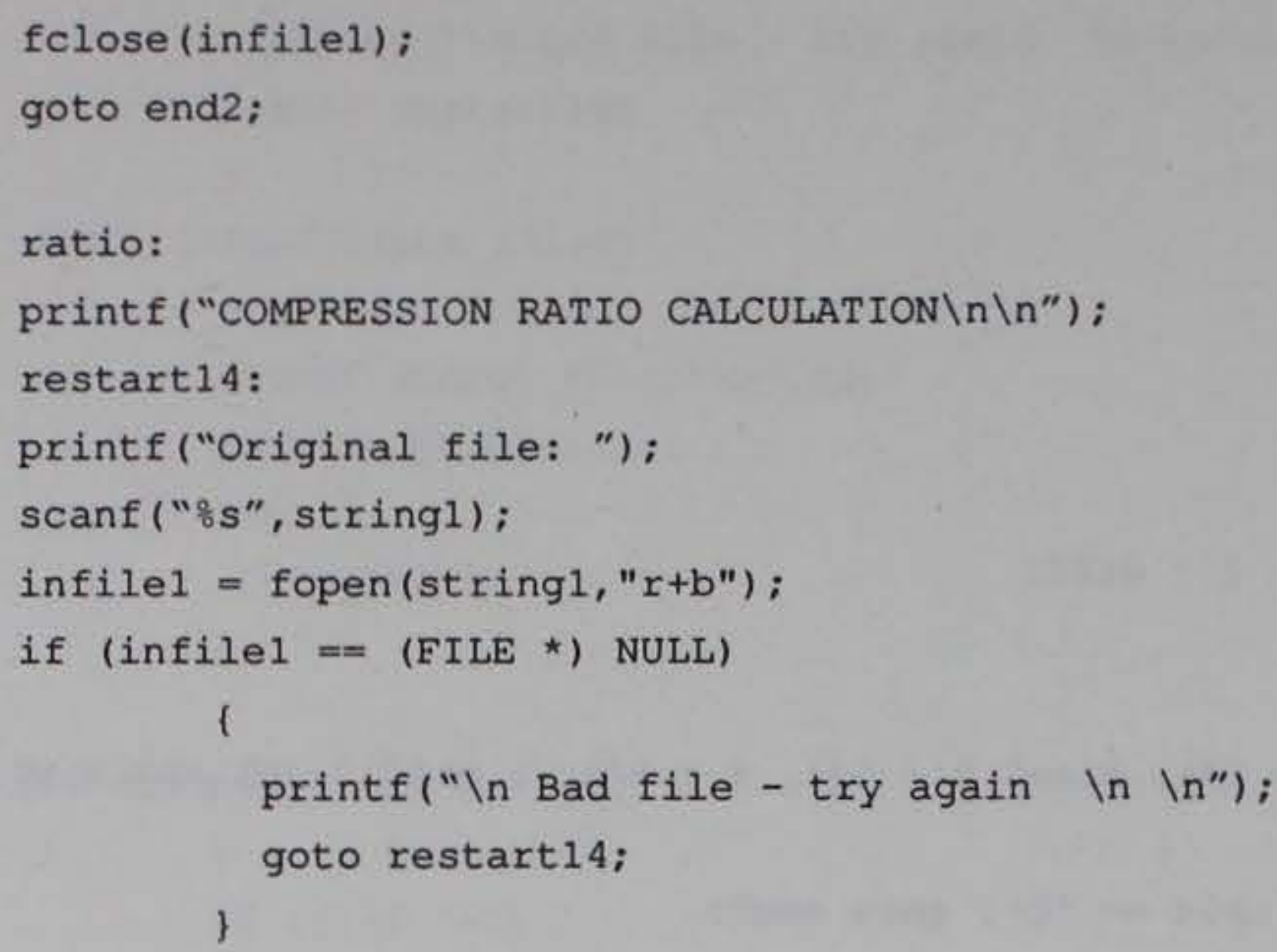




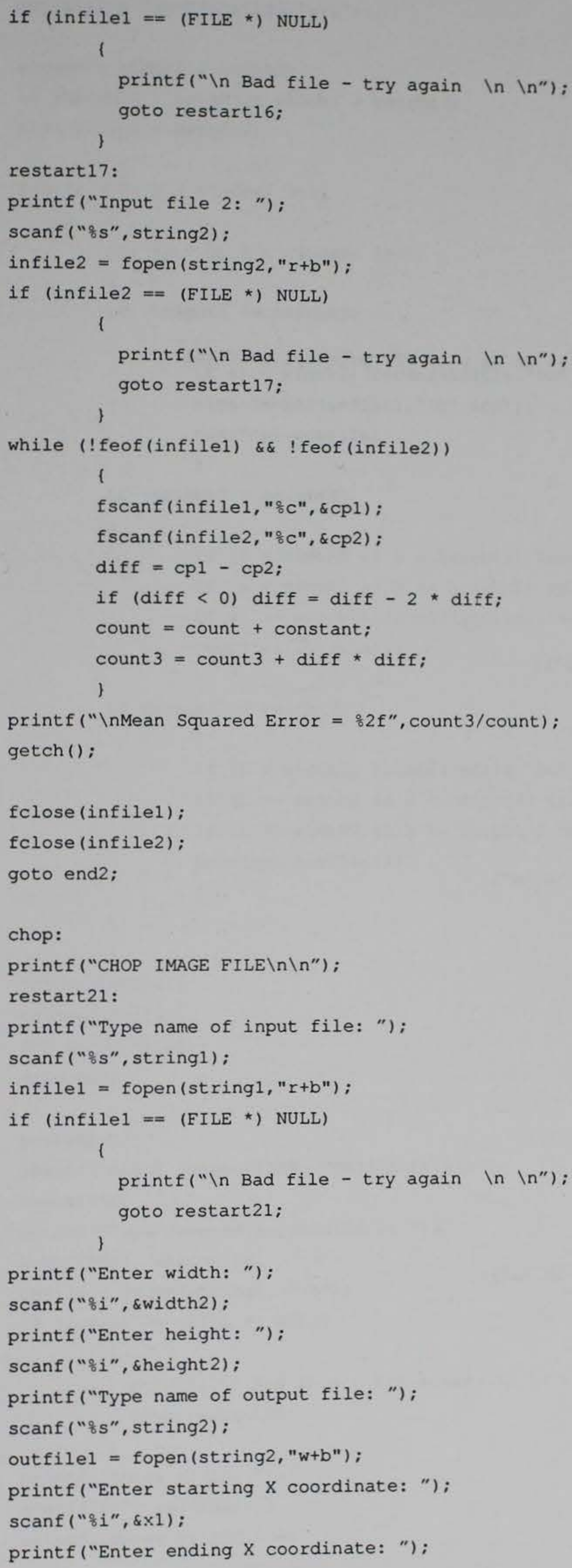




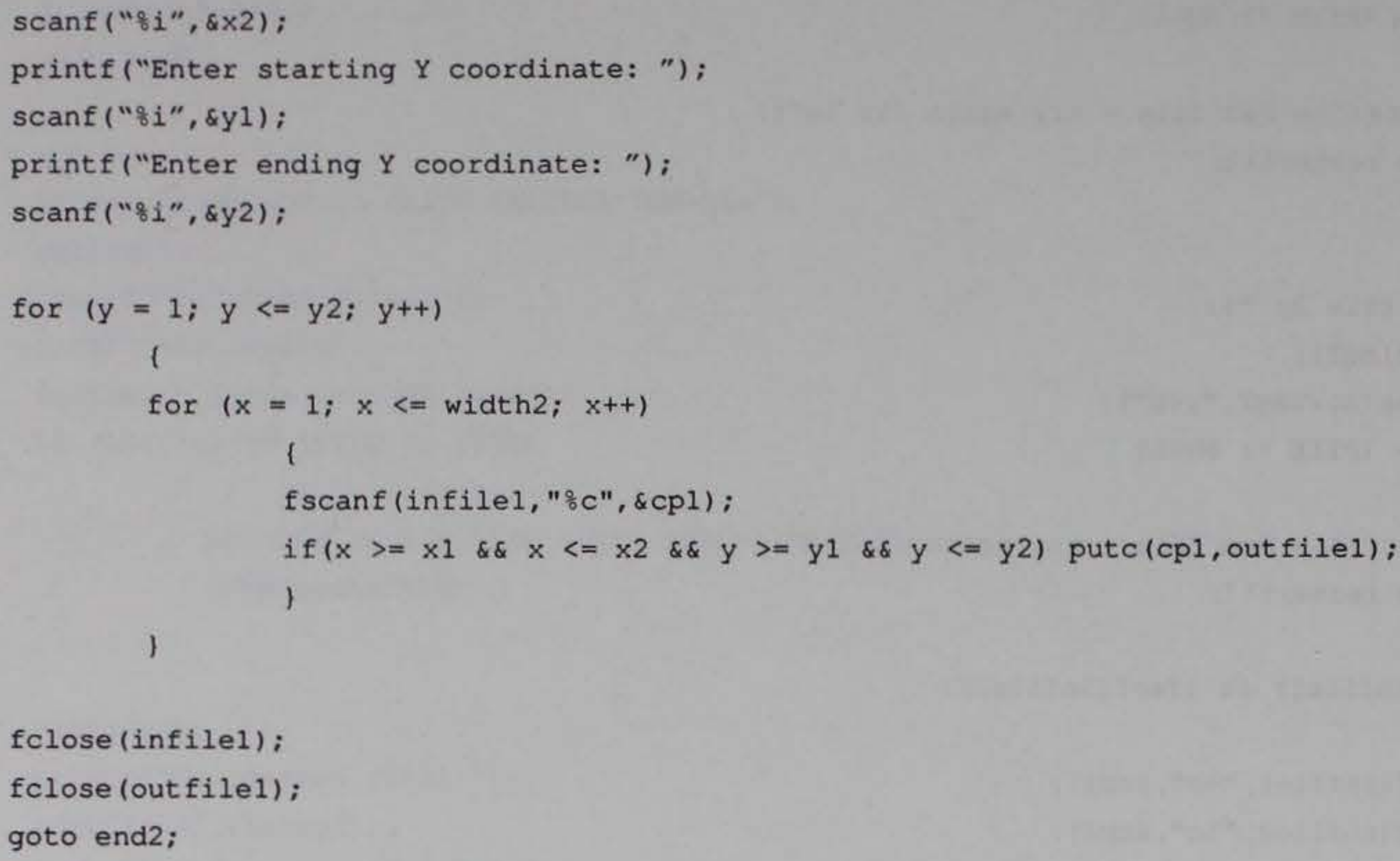




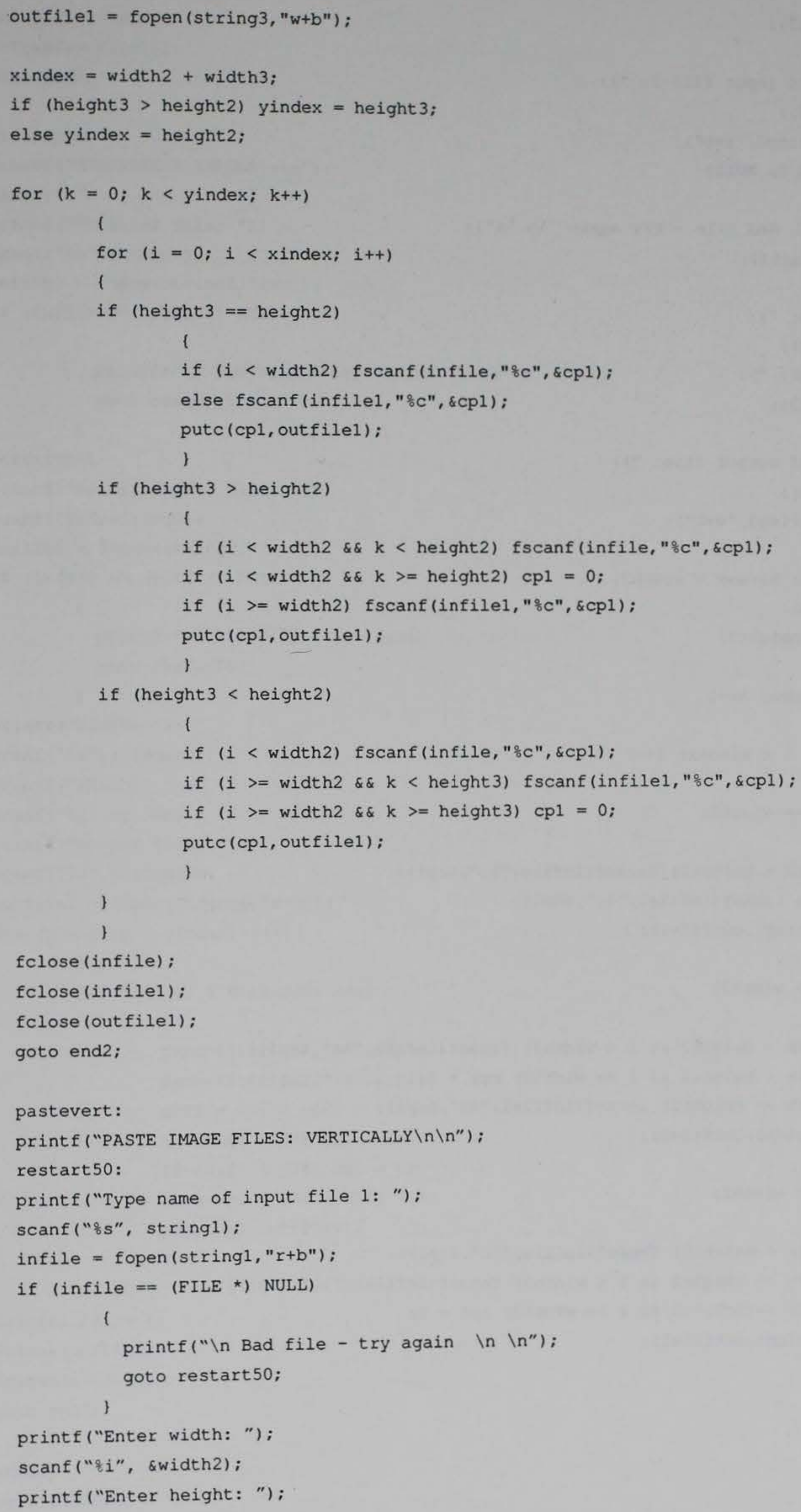




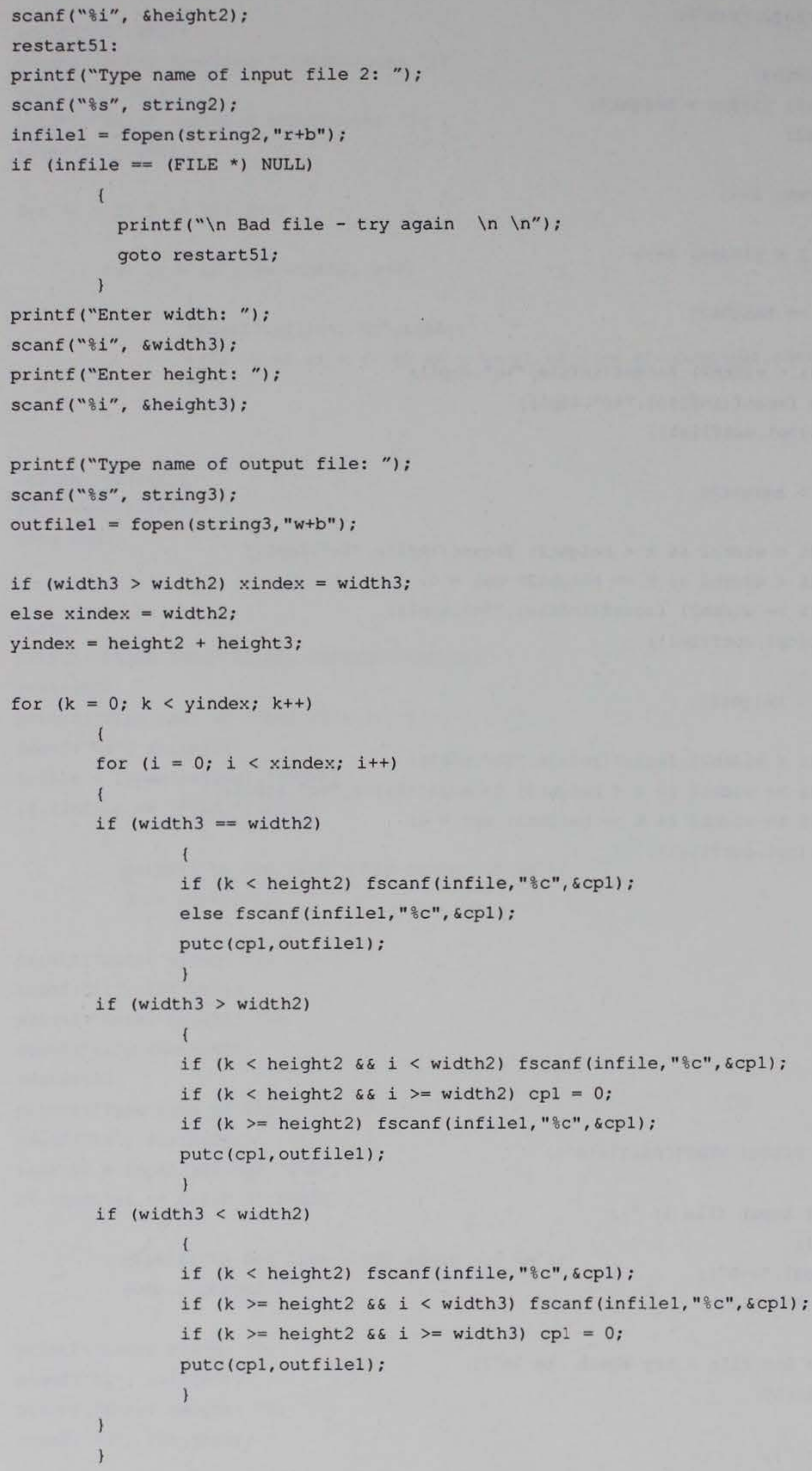




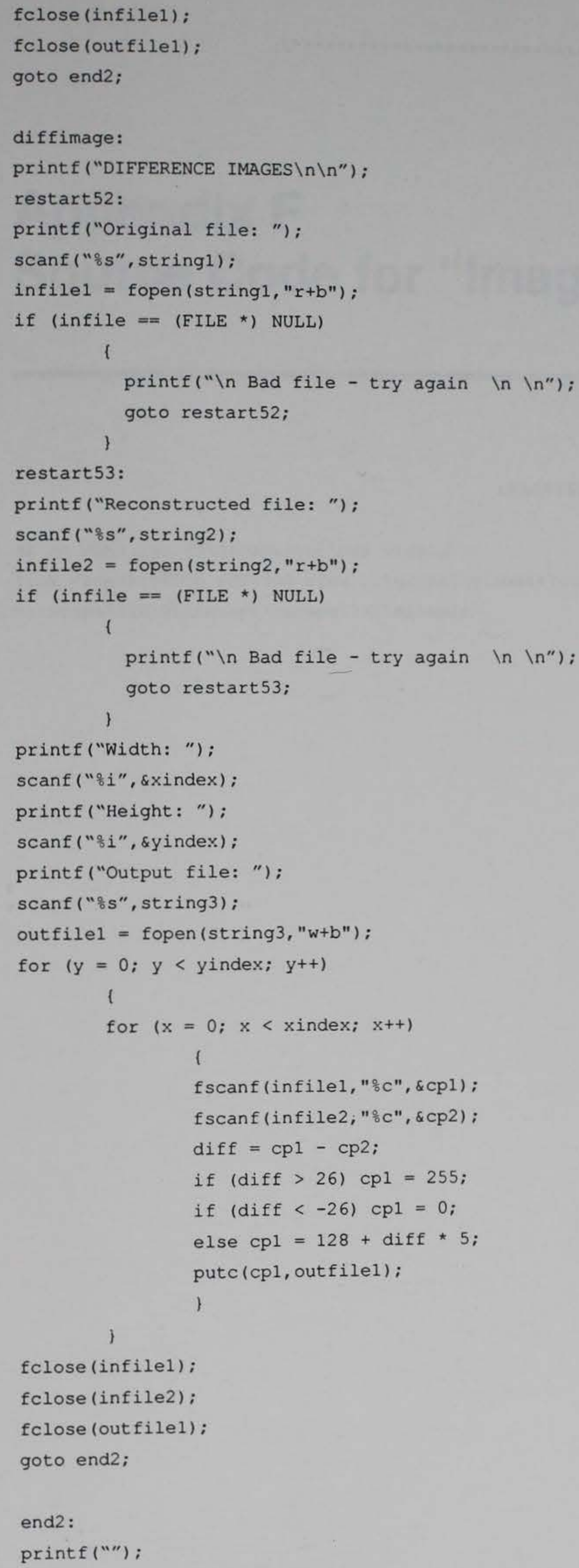


1 


\section{Appendix F Source Code for "Image View" Software}

IMAGVIEW.BAT

cl /C /DM5 /AL /I \cscapelinclude view.c

link/stack: 44000 /SE: 300 view, , Iglobal/global+\standard standard

+ \cscape $\backslash 1$ ib $\backslash$ m1lcscap+ $\mid c s c a p e \backslash l i b \backslash m 1 l o w l$; 
VIEW. C

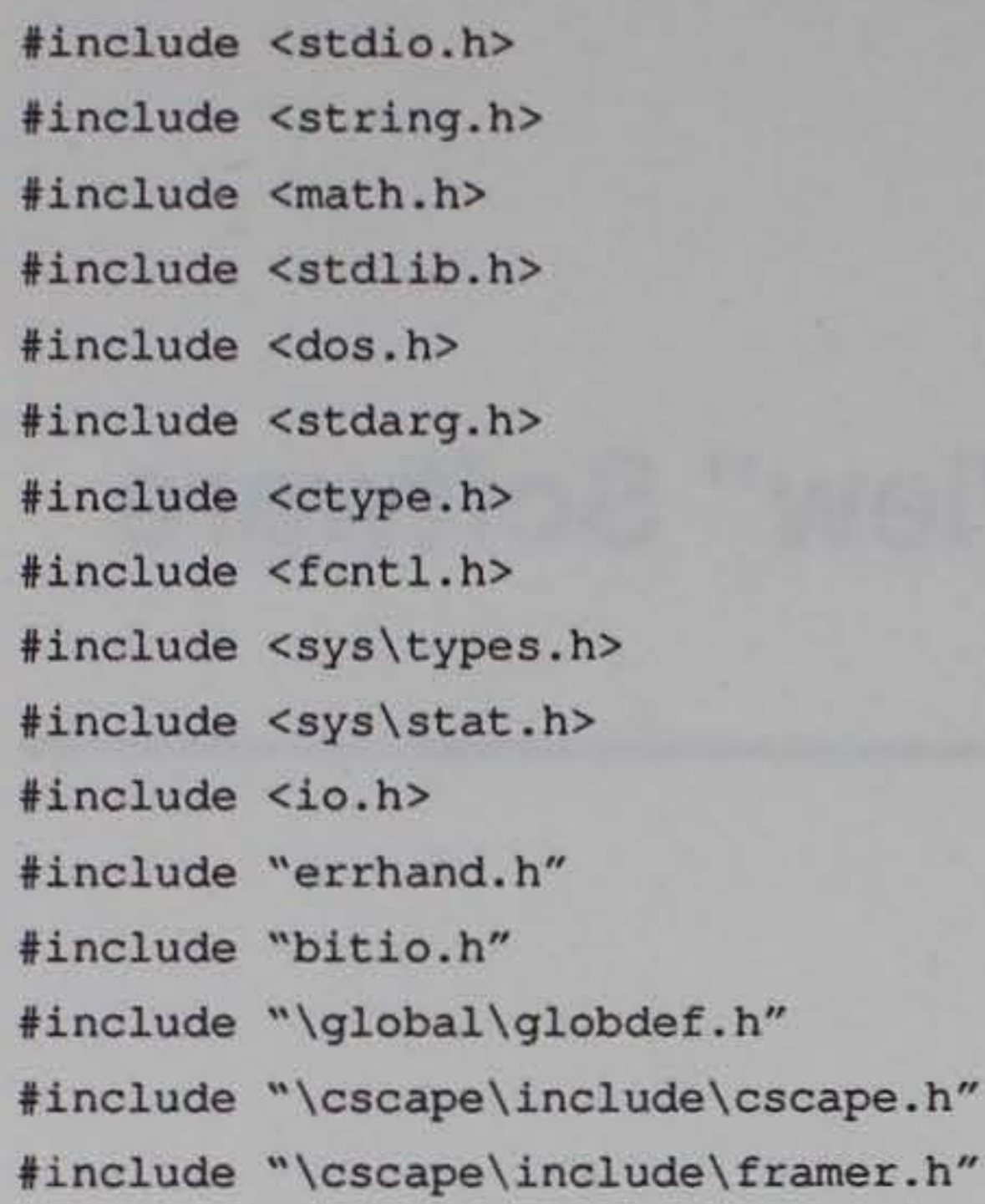

int vga_code, graphics, width, height, mode, modes [5], choice,

read_bank, write_bank, top, stat_buf [4], radius,ix, iy, count;

char instring $1[80]$, message_string [81], string [80], string $3[20]$;

unsigned stringpal1 [768], colorflag=2;

sed_type frame;

FILE *inpalette;

FILE *input_file;

FILE *input_filel;

void graphics1 (idata, mask, ch)

int idata, mask, ch;

i

int $i, j, p, 1, m, i i, j j, k k, m m, \operatorname{red} 2[8], \operatorname{green} 2[8], b]$ ue $2[8], x, y$;

unsigned char red[256], green[256], blue [256], redl[256], green1[256],

blue1[256], point_color, cp, ch3;

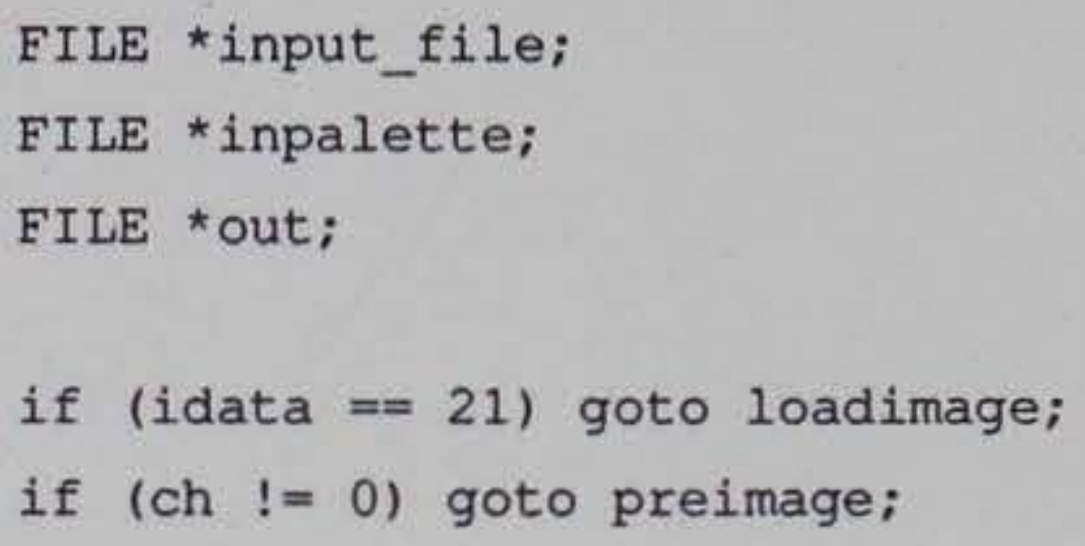

loadimage:

Set_Mode (3) ;

restart19:

printf ("Type name of image series file: ");

scanf ("ㅇs", string); 


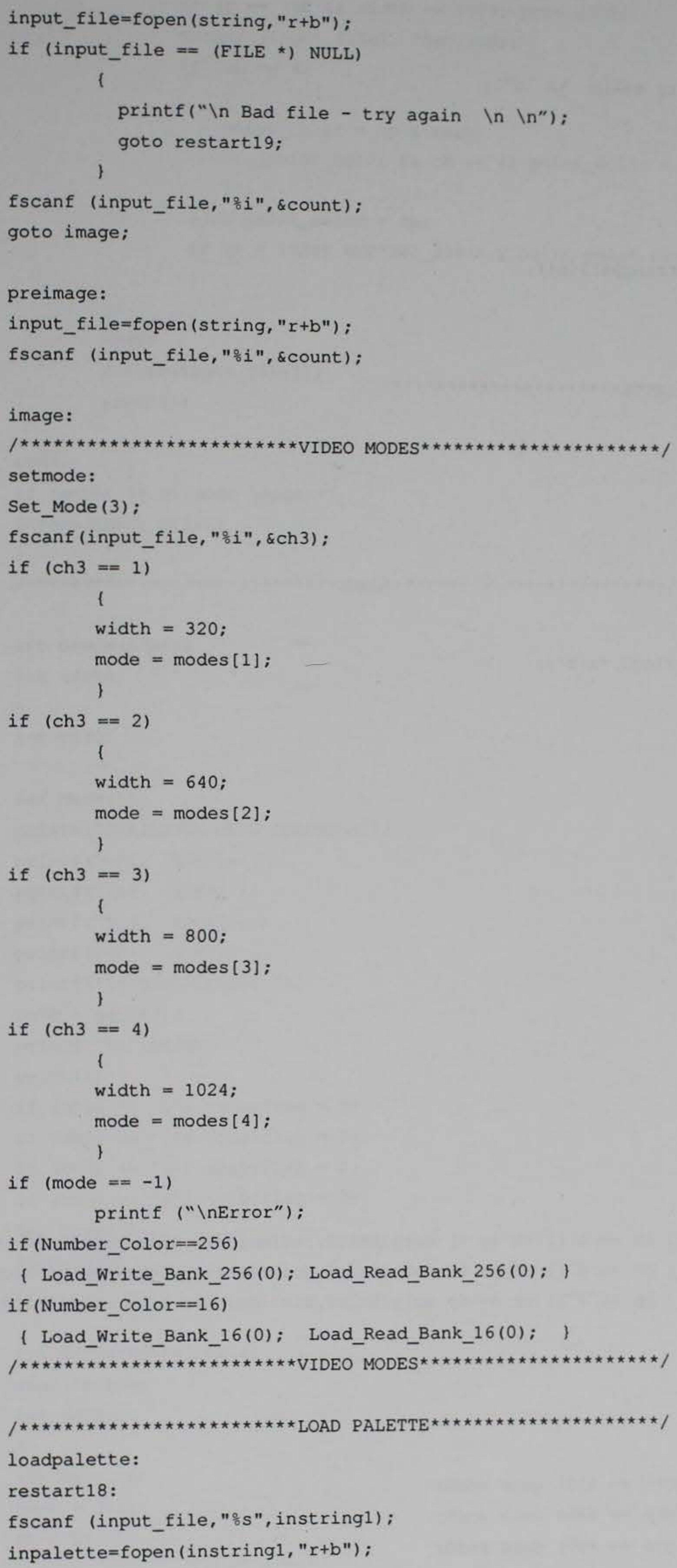




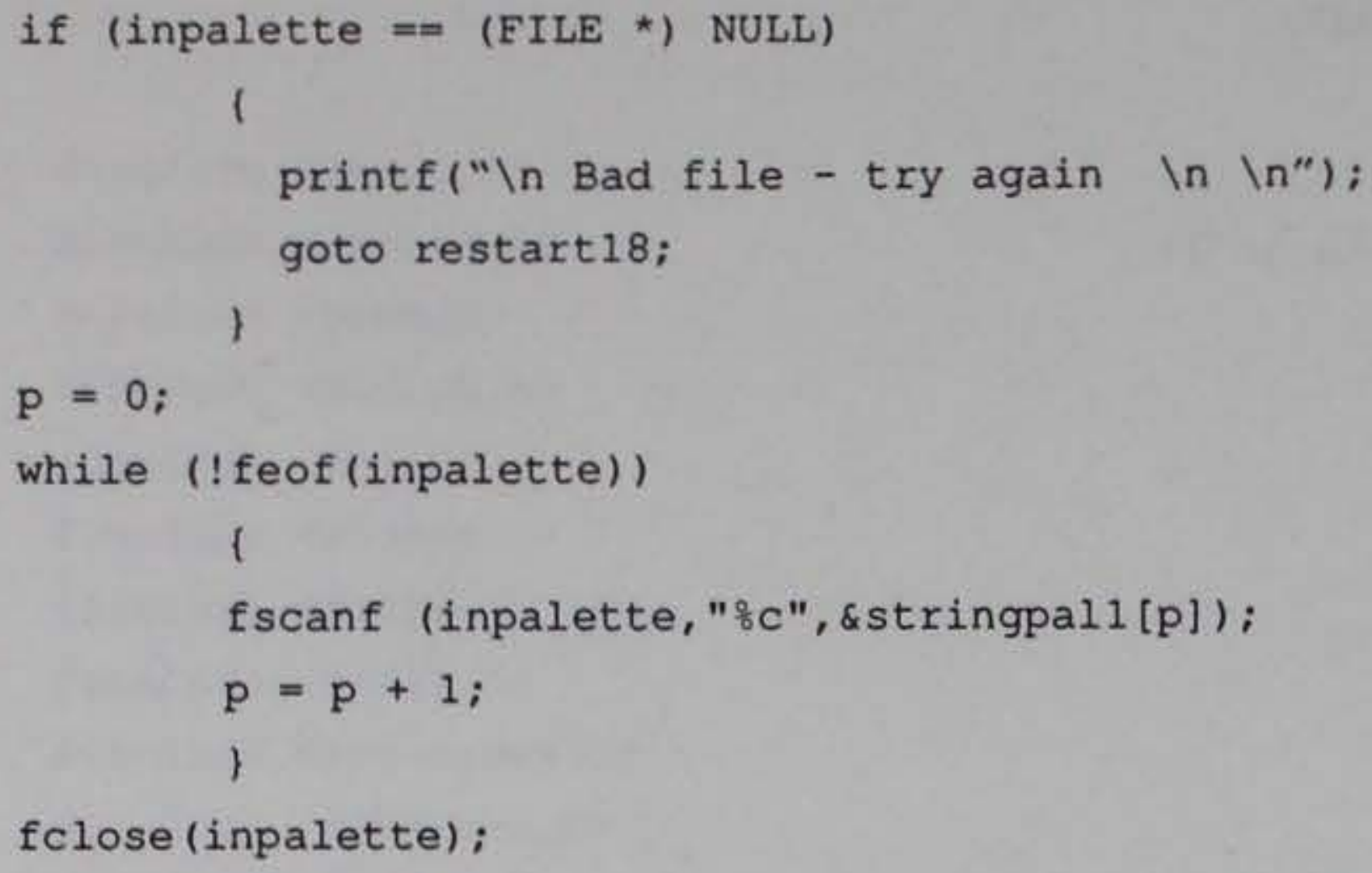




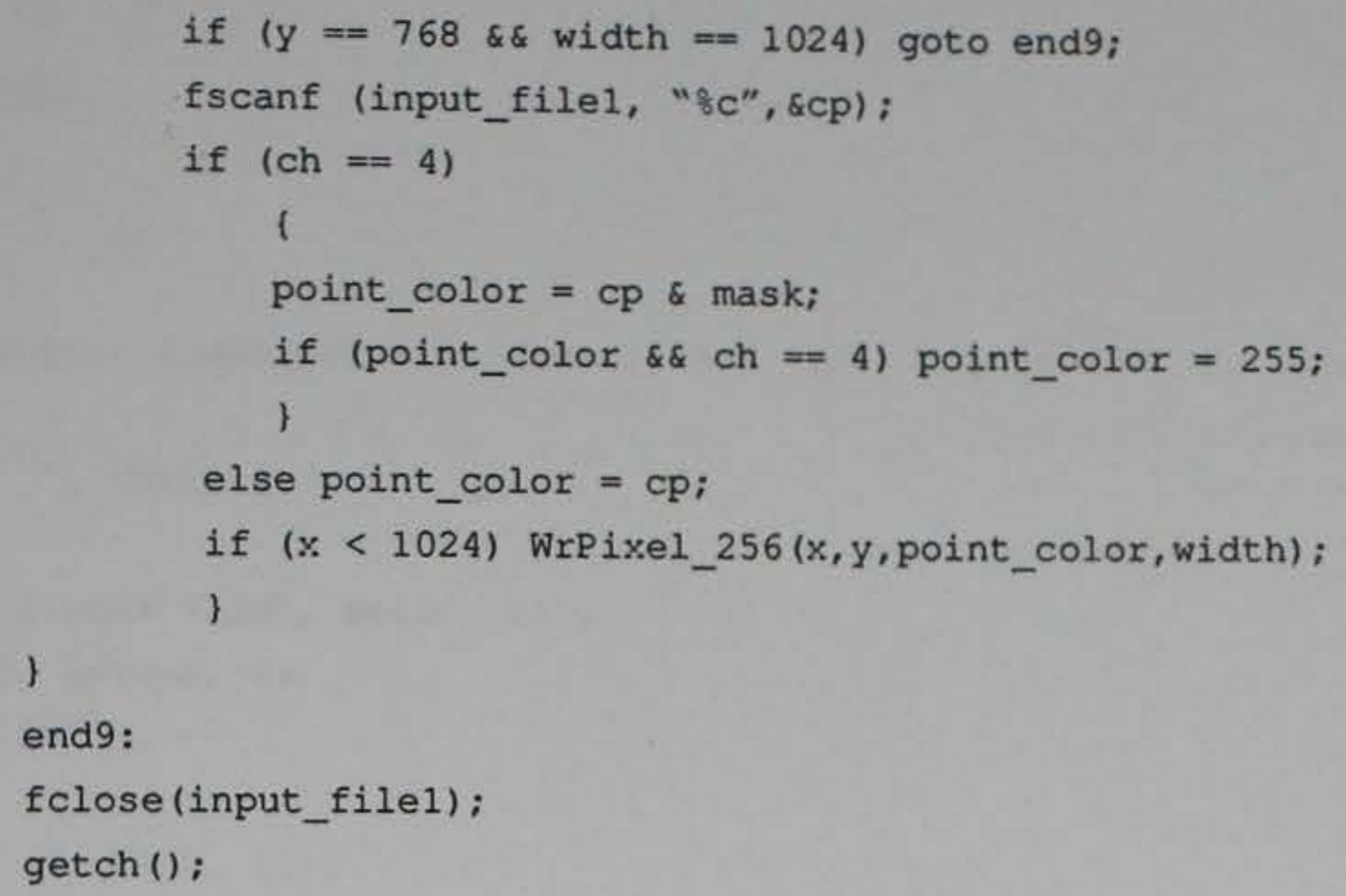




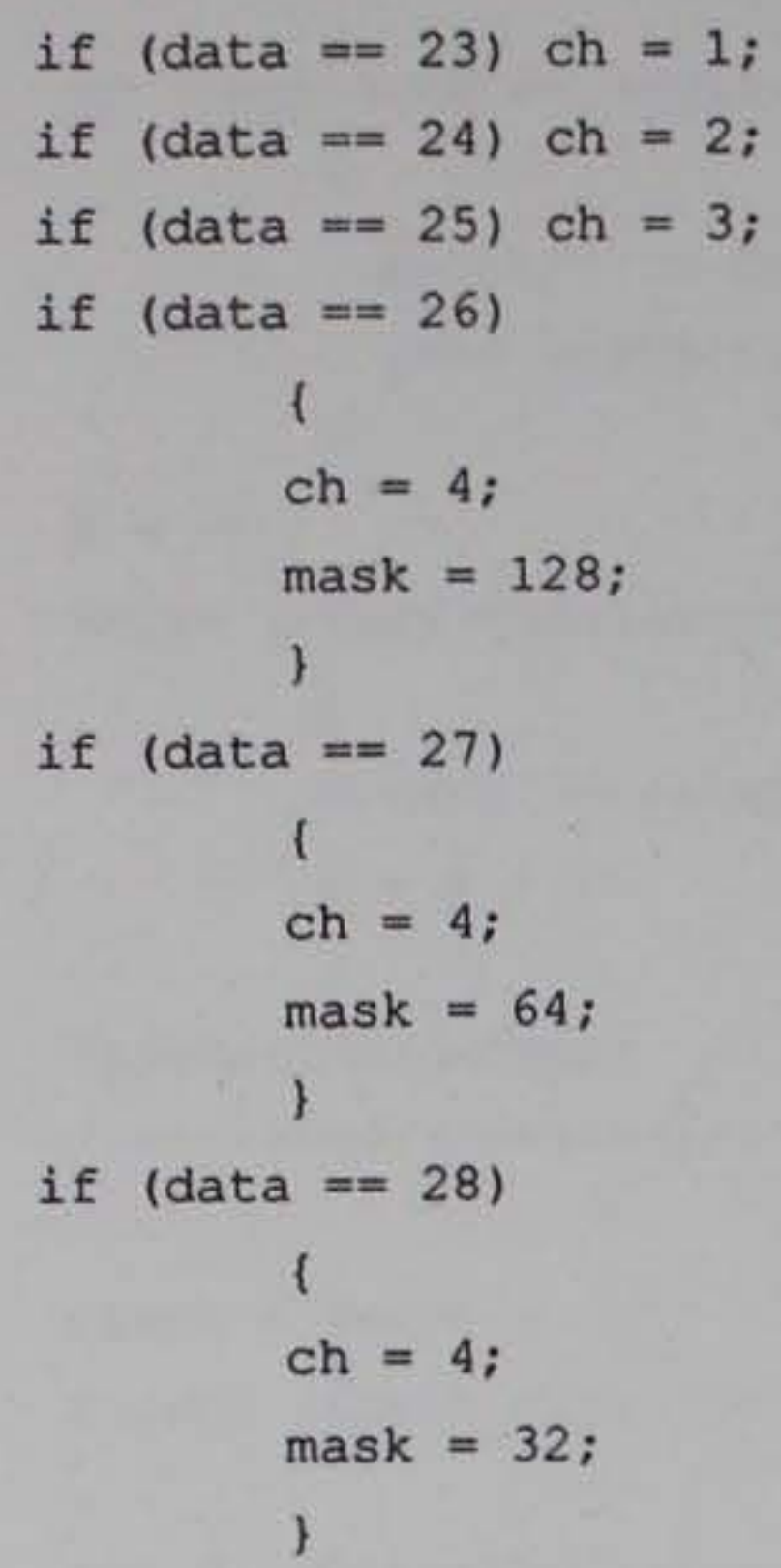




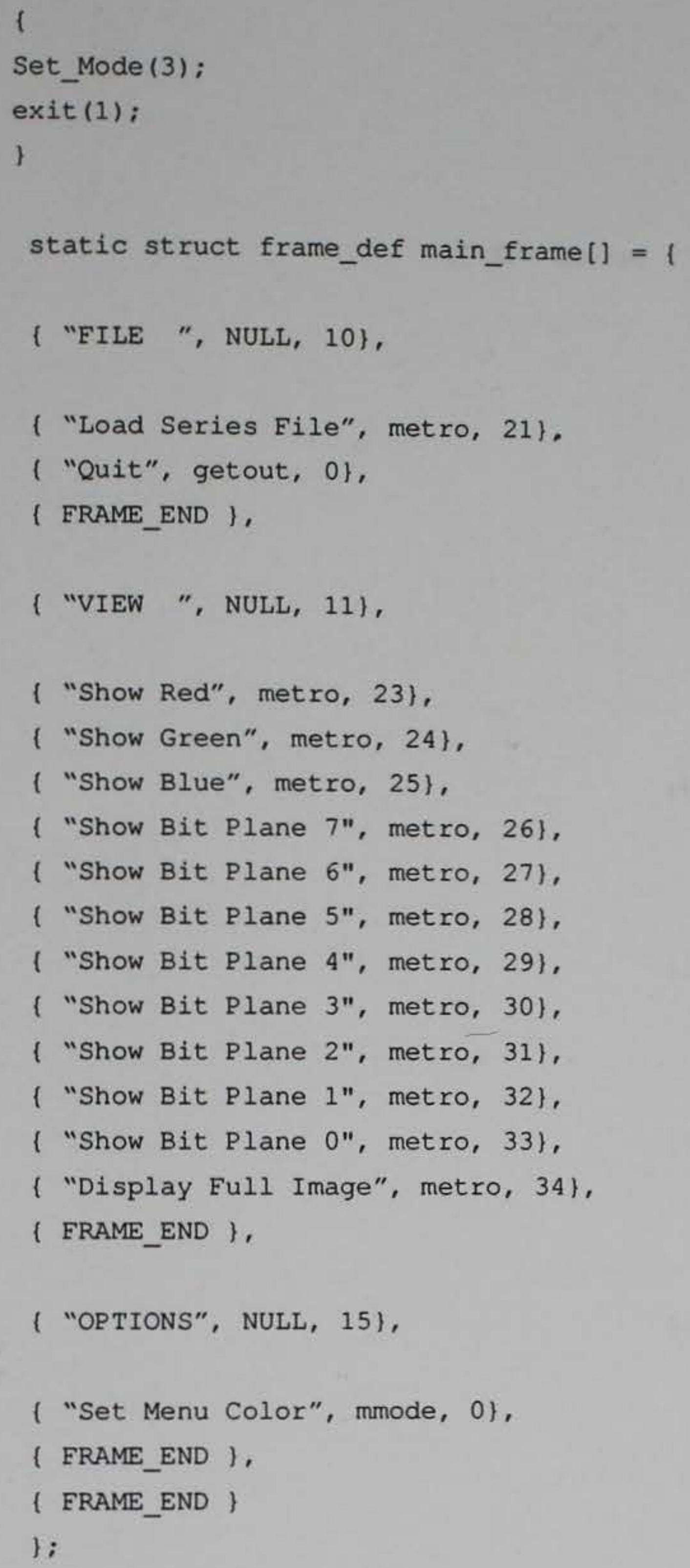




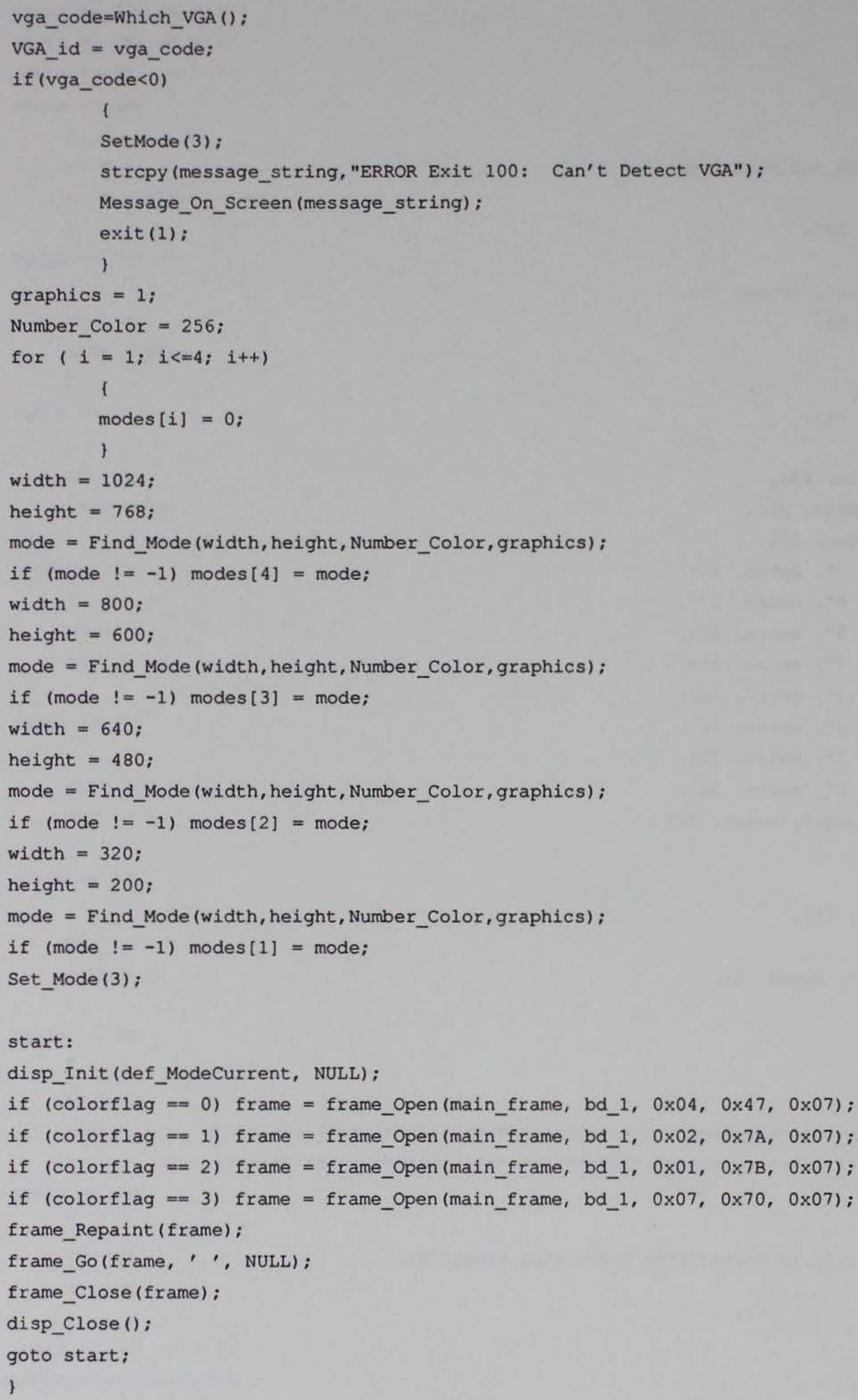

